\title{
Deep limit order book events dynamics*
}

\author{
Yann Bilodeau ${ }^{1}$
}

December 2, 2020

\begin{abstract}
This paper analyzes the limit order book events arrival dependency structure using highdimensional Hawkes processes. We seek for recurrent relationships among events from a set of 86 event types which in addition to transactions, includes limit order submissions and cancellations taking place up to the 20th depth level of the order book. We focus on BMW, SAP, and ADS, three liquid DAX 30 index stocks for which we have a microsecond stamped high-frequency dataset covering the 61 trading day period going from February 1 to March 31, 2013. For each stock, we build a tailored descriptive model by selecting recurrent events relationships. Estimated on a daily basis, we find that the selected models offer interesting data fitting performance, particularly for limit order submissions and cancellations occurring on the first five price levels of the order book. Finally, we use the comprehensive sets of estimated parameters to describe a global events arrival dynamics that we relate to the potential behaviors of market participants having different objectives and directional views.
\end{abstract}

\section{JEL classification: C22 C41 C53 G11}

Keywords: Limit order book, Hawkes process, high-frequency, algorithmic trading, liquidity, Frankfort Stock Exchange.

* Research supported by SSHRC Canada, FRQSC Quebec, Canada Foundation for innovation and the Canadian Derivatives Institute. The author thanks professors Georges Dionne and Manuel Morales, as well as Gabriel Yergeau, Xiaozhou Zhou and Mathieu T-Blais for their support and comments.

1 Canada Research Chair in Risk management and Finance Department, HEC Montreal. yann.bilodeau@hec.ca. 


\section{Introduction}

In recent years, technology improvements have completely transformed the financial markets landscape. The main part of market activity is now performed through algorithms on electronic trading platforms using real-time open limit order book information. Even more conventional investors such as banks, mutual funds and institutions now outsource their trading tasks to algorithmic traders who split the main orders into multiple child orders distributed over time, trying to achieve the best execution price while hiding their intention to other market participants. In this new financial world, speed and information quality have become very important keys to success. Indeed, in order to remain competitive, algorithms must take investment decisions and send their answer to the market in a few milliseconds time frame and this window size tends to decrease years after years with technological improvements. Although high-frequency and algorithmic trading now represent a market standard around the world, limit order book modeling has not yet received a very important coverage in academic and scientific literature. This situation suggests a gap between practical and academic knowledge on this topic, which is not surprising from an economic point of view. Indeed, the high competition level and important barriers to entry probably encourage practitioners to keep the secret of their successful models and strategies. In this context, this paper focuses on modeling the behavior of a multilevel limit order book at a microscopic level with algorithmic trading perspectives.

Until recently, regularly spaced data were used for asset returns modeling and forecasting purposes. However, to maximize the advantage of newly available high frequency data, modeling irregularly spaced data is essential since at a microscopic level, important market events such as transaction and limit orders submission are irregularly spaced in time. In this situation, point processes models are natural candidates to describe these irregularities. In a widely cited paper, Cont, Stoikov et al. (2010) use a Poisson process system to represent events arrival in a limit order book and perform various quantities computations, such as the probability of making the spread over a given period. Despite the interesting results produced by their model, the main disadvantage of homogeneous Poisson process remains the assumption of independent exponentially distributed event arrival times. More recently Huang, Lehalle et al. (2015) and Muni Toke and Yoshida (2017) have developed and simulated complete limit order book models based on state-dependant event arrival processes. Although the former introduces a certain level of dependency between processes, 
none of these papers explicitly consider the arrival of events itself as a major factor driving the arrival of other events. However, it has been established that a clustering phenomenon is present in the arrival sequence of some types of limit order book events. This particularity led to the introduction of self-exciting point processes in finance such as the Hawkes process, that was originally used for earthquakes occurrence modeling and forecasting (Hawkes (1971), Hawkes and Oakes (1974)).

As well described by Shek (2011), in a self-exciting process such as univariate Hawkes model, the current arrival rate for a given type of events is driven by past occurrences of events of the same type. In its multivariate version, Hawkes process is said to be a cross or mutually-exciting process. Within this extended case, in addition to past events of the same type, event arrival intensity is also driven by past events of other types. Using this feature, it becomes possible to account for dependency between different event categories occurring at irregularly spaced times, which has otherwise, not been explicitly covered in the current literature.

Over the last years, Hawkes processes themselves have been used for various purposes in finance. A very general definition of this process and its possible financial applications is provided by Embrechts, Liniger et al. (2011). From another point of view, Bacry, Delattre et al. (2013) use them for price changes modelling purposes. They model the number of ticks up and down for both single and pairs of assets using counting processes. Hawkes processes allow them to take autocorrelation in price movements into account. Fauth (2012) use four bivariate mutually-exciting processes to model bid and ask prices moves up and down. Their processes pairs modeling structure allows them to produce a consistent bid-ask spread. In order to add more information to their model, they also include trades volumes. Large (2007) uses up to ten Hawkes processes to analyse the limit order book resiliency well-known phenomenon.

In a market events perspective closer to the limit order book, Hewlett (2006) uses bivariate Hawkes processes for buy and sell trades occurring on the FX markets modelling. He then applies them for optimal trading strategies purposes as developed by Almgren (2003), Almgren (2000), Bertsimas and Lo (1998), Obizhaeva and Wang (2012) and more recently Cheng, Di Giacinto et al. (2017), assuming the market maker considers buy and sell orders arrival to follow Hawkes processes. Shek (2011) also uses a bivariate mutually-exciting model for buy and sell trades occurrence. As Fauth 
(2012), he includes trade sizes in order to consider more information in large trades than in small ones. Toke and Pomponio (2012) apply a bivariate process to model the occurrence of trades consuming more than one order book level, also known as trade-through. Finally, Bowsher (2007) uses two pairs of bivariate Hawkes processes to model the joint arrival of trades and mid-quote changes.

In their respective thesis, Vinkovskaya (2014) and Huang (2012) use multivariate Hawkes processes in order to model the limit order book first level order flows. Vinkovskaya (2014) considers four different processes representing limit order arrivals and market order arrivals for both sides of the book. For estimation procedure simplification, she combines market order and limit order cancellation events. She presents a regime switching extension in order to account for the spread size effect on the order arrival intensities. She performs her parameters estimation using a 2008 subsample of the Trade and Quotes (TAQ) database with a one second time precision. The forecasting power of her model is interesting since it outperforms Poisson model and AR, MA and ARIMA time-series models for out-of-sample predictions. On the other hand, Huang (2012) uses six multivariate Hawkes processes in order to model the arrival of market orders, limit orders and limit order cancellations on both sides of the book. Parameters estimates are obtained from a millisecond precision order book snapshots dataset on a five-day period in 2009 for Vodaphone (VOD.L), a stock traded on the London Stock Exchange. He then computed various probabilities using a Monte Carlo simulation method based on the thinning algorithm introduced by Ogata (1981).

Since only few of the previously cited contributions have analyzed more than one level in the limit order book, we propose to extend the current literature in this way. In this context, we suggest a multivariate Hawkes processes system to model and analyze the behavior of a multilevel limit order book. Our main objective is to determine how various order book event types occurring at different levels affect each other. Let us assume a first level that contain only one limit order and the absence of hidden or iceberg orders. By the nature of the limit order book itself, one can easily see that the cancellation of this order or the submission of a market order consuming it entirely would instantly convert the current level 2 into the new best bid or best ask level. In the same way, level 3 would become level 2 and so on. With this simple example in mind, it is possible to believe that analyzing higher levels event arrival processes could provide interesting information on the 
future states of best price levels where transactions generally occur. To accomplish this task, we define an extensive set of events occurring on the first twenty levels of both sides of the book and take advantage of the Hawkes dependency structure to establish how they relate to each other.

Our parameters estimation and performance analysis are realized on a Xetra 2013 microsecond (10-6) precision limit order book dataset. It is possible to expect the microsecond precision to provide an informational advantage over what has been done in the past. However, the real Xetra order flows are not directly available and have to be deduced using observable limit order book state changes and executed trades. This procedure may lead to some missing events because of the aggregated nature of the data. On the other hand, our dataset is exactly the one used by algorithmic trading systems that were operating on the Frankfurt Stock Exchange over the February to March 2013 period, suggesting we cannot get closer to real-time market information without having access to the stock exchange internal database. This fact is important since our models are developed in an algorithmic trading perspective. Indeed, we have to keep in mind that it is possible for an algorithmic response to an order book event to be so fast that it could not have been though and launched by a human trader. Fortunately, Hawkes processes have the capacity to capture this type of very short-term phenomena.

The rest of the paper is organized as follows: In Section 2, we present the concept of Hawkes processes, which provides the theoretical foundations for this paper. In Section 3, we introduce the dataset. In Section 4, we define the set of events considered as potentially interrelated. In Section 5, we use Section 2 theory to specify our complete model. In Section 6, we elaborate the estimation methodology that will lead to one descriptive model for each of our three liquid stocks. In Section 7, we analyse the results of the estimation phase in terms of models selection, data fitting and estimated parameters characteristics. In Section 8, we use the estimated parameters to describe a global events arrival dynamics that we relate to market participants potential behaviors. Finally, in Section 9, we conclude and introduce an extension for this paper.

\section{Hawkes processes}

In this section, we present the theoretical foundations underlying to our models, their estimation methodology, and the analysis performed on the results. Before presenting the fundamentals for univariate and multivariate Hawkes processes models, we introduce the general concept of point 
process, which is defined by Carstensen (2010) as a "statistical model used to describe point patterns in a given space". In our financial context, these points correspond to events arrival times. In general terms, a point process $\{N(t), t \geq 0, N(t) \in \mathbb{N}\}$ counts the events occurring at times $\left\{\left\{t_{i}\right\}, i=1, \ldots, N(t)\right\}$. The theoretical behavior of this mathematical concept is dictated by the form taken by its intensity function $\lambda(t)$. As an example, the intensity of a pure homogeneous Poisson process, hereafter referred as Poisson process, is provided by $\lambda(t)=\lambda$, a constant parameter corresponding to the average number of event occurrences by unit of time. The following expression defines a point process compensator.

\begin{tabular}{|l|l|l|}
\hline$(1)$ & $\Lambda\left(t_{0}, t_{1}\right)=\int_{t_{0}}^{t_{1}} \lambda(t) \mathrm{dt}$ \\
\hline
\end{tabular}

Since $\lambda(t)$ is the arrival rate function, assuming $t_{1} \geq t_{0}, \Lambda\left(t_{0}, t_{1}\right)$ may be interpreted as the time period $t_{0}$ to $t_{1}$ expected number of event occurrences. ${ }^{2}$ Equation (2) provides the general loglikelihood function, which can be maximized to estimate any point process model parameters.

$\begin{aligned} \ln \mathcal{L}\left(\left\{t_{i}\right\}_{i=1, \ldots, N(T)} \mid \theta\right) & =\int_{0}^{T}(1-\lambda(s \mid \theta)) d s+\int_{0}^{T} \ln \lambda(s \mid \theta) d N(S) \\ & =T-\int_{0}^{T} \lambda(s \mid \theta) d s+\sum_{t_{i}<T} \ln \lambda\left(t_{i} \mid \theta\right) \\ & \equiv-\Lambda(0, T \mid \theta)+\sum_{t_{i}<T} \ln \lambda\left(t_{i} \mid \theta\right)\end{aligned}$

\footnotetext{
${ }^{2}$ The Poisson process compensator function is defined as $\Lambda\left(t_{0}, t_{1}\right)=\int_{t_{0}}^{t_{1}} \lambda \mathrm{dt}=\lambda\left(t_{1}-t_{0}\right)$
} 


\section{Univariate Hawkes process}

Having defined the general concept of point process, we now introduce the Hawkes processes model. In its univariate version, such model assumes the occurrence of an event to have an impact on the arrival of events of the same type. Under this paradigm, by its effect on the process intensity function, an event occurrence increases the probability of another occurrence that does the same and so on. Consequently, the process has the potential to become self-excited, which makes the description of events clustering phenomena possible.

As defined by Hawkes (1971) and Hawkes and Oakes (1974), in its most general form, the selfexciting Hawkes process $\{N(t), t \geq 0, N(t) \in \mathbb{N}\}$, is characterized by the following continuous intensity function:

\begin{tabular}{|l|l|}
\hline$(3)$ & $\lambda(t)=\mu(t)+\int_{s<t} v(t-s) d N(s)$ \\
\hline where \\
$\lambda(t):$ process intensity \\
$\mu(t) \geq 0:$ baseline intensity \\
$v: \mathbb{R}^{+} \rightarrow \mathbb{R}^{+}:$excitation kernel function
\end{tabular}

The previous Poisson process with constant intensity $\lambda$ represents a special case where $\forall t \in \mathbb{R}^{+}$, $v(t)=0$ and $\mu(t)=\lambda$.

Because of discrete nature of $N(t)$ that counts the events taking place at times $\left\{\left\{t_{i}\right\}, i=\right.$ $1, \ldots, N(t)\}$, it is possible to rewrite (3) :

$$
\lambda(t)=\mu(t)+\sum_{t_{i}<t} v\left(t-t_{i}\right)
$$

As it is often the case in financial and economics literature, we use a constant baseline intensity $\mu(t)=\mu$. We also use the simple exponential kernel function, which is characterized by the following expression: 


\begin{tabular}{|l|l|}
\hline$(5)$ & $v(t)=\alpha e^{-\beta t}$ \\
where & \\
$\alpha>0:$ excitation term & \\
$\beta>0$ & exponential decay factor \\
\hline
\end{tabular}

Combining (5) and (4), the univariate exponential Hawkes process intensity function is defined as:

\begin{tabular}{|l|l|}
\hline (6) & $\lambda(t)=\mu+\sum_{t_{i}<t} \alpha e^{-\beta\left(t-t_{i}\right)}$ \\
\hline
\end{tabular}

In general terms, $\alpha$ represents the immediate impact of an event occurrence on its arrival rate. Each time an event occurs, the process intensity is instantaneously incremented by $\alpha$. Because of the kernel function, this effect also immediately begins to decline. Considering an event taking place at time $t_{i}$, the proportion of $\alpha$ still present in the process intensity at time $t>t_{i}$ is given by $e^{-\beta\left(t-t_{i}\right)}$. Thus, this quantity depends on both the exponential decay factor $\beta$ and the time elapsed between $t_{i}$ and $t$. It is important to keep in mind that under this exponential framework, an event effect never completely vanishes from the process intensity. However, it remains practically significant over a time span whose length is dictated by $\beta$, which brings us to the definition of the half-life period as presented in (7).

$$
H L=\frac{\ln (2)}{\beta}
$$

Widely used in biology and physics, this measure is interpreted as the duration over which an effect loses $50 \%$ of its strength. It actually loses $99 \%$ of its strength after about 6.64 half-live periods. For computational purposes, we consider it to completely vanish after 20 half-live periods. Back to (6), it is possible to denote that occurrences effects are cumulative. Indeed, $\lambda(t)$ theoretically encompasses the residual effects of all the events having taken place at times $\left\{t_{i}\right\}, i=$ $1, \ldots, N(t)$. The process intensity also includes $\mu$, the baseline intensity. This constant represents the event arrival rate in place before the occurrence of the first event. It may also play an important role in the rate prevailing between self-excitation periods when past events effects on $\lambda(t)$ have become marginal. 
The following expression defines the branching ratio $(B R)$, which may be interpreted as the average number of child events expected to follow the arrival of a parent event.

(8) $\quad B R=\int_{0}^{\infty} \alpha e^{-\beta t} d t=\frac{\alpha}{\beta}$

This expression leads to condition (9), which ensures the process to be stationary. This condition is intuitive since it is easy to see that a branching ratio equal or larger than one could lead to a process explosion. In such case, it would be expected for each event occurrence to lead to more than one new occurrence.

$$
\frac{\alpha}{\beta}<1
$$

The branching ratio is also an important component of the process intensity unconditional expectation, which is provided by the following expression:

\begin{tabular}{|l|l}
\hline$(10)$ & $E[\lambda(t)]=\frac{\mu}{1-\frac{\alpha}{\beta}}$ \\
\hline
\end{tabular}

The following expression presents the self-exciting Hawkes process compensator function :

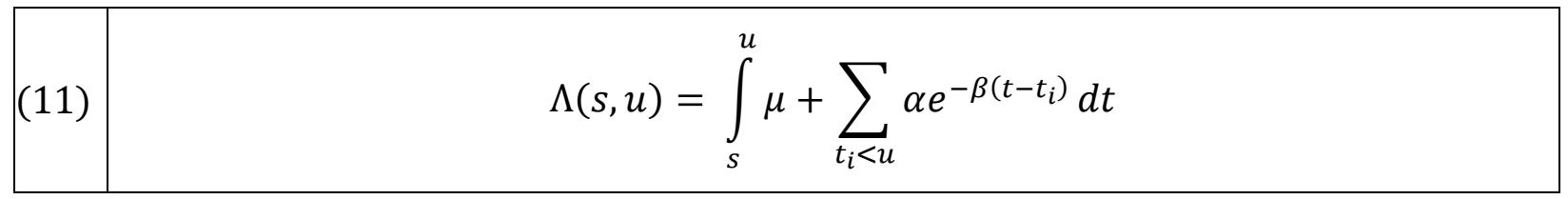

The first term of the integral relates to the baseline intensity while the second cumulates the past events residual effects. Thereafter, given the desirable properties of the exponential Hawkes intensity function, the compensator closed form solution is provided by the next expression: 


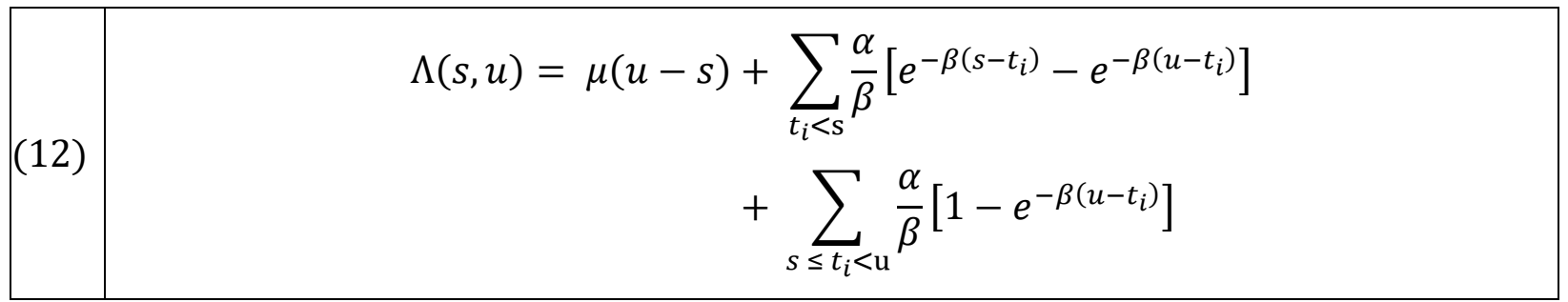

Finally, applying (6) and (12) to (2) leads to the univariate exponential Hawkes process loglikelihood function, which, when computed for the period going from times 0 to $T$, is provided by the following expression :

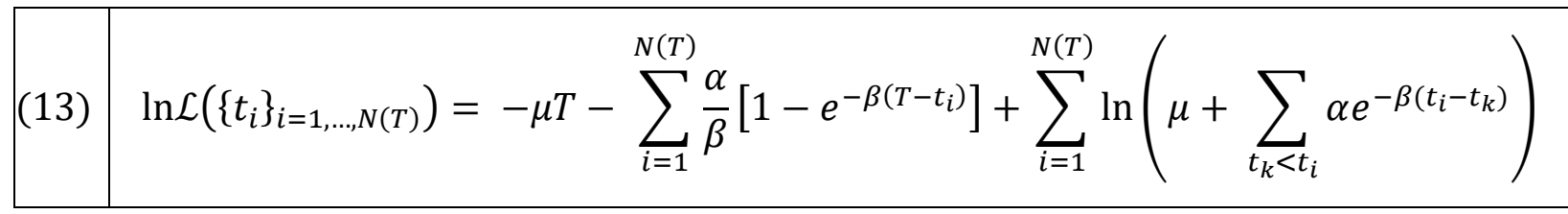

Since inefficient from a computational point of view, it is transformed into the following expression involving the recursive function $R(i)$, which is appropriate for the purposes of numerical maximization.

\begin{tabular}{|l|l|}
$\ln \mathcal{L}\left(\left\{t_{i}\right\}_{i=1, \ldots, N(T)}\right)$ & $=-\mu T-\sum_{i=1}^{N(T)} \frac{\alpha}{\beta}\left[1-e^{-\beta\left(T-t_{i}\right)}\right]+\sum_{i=1}^{N(T)} \ln (\mu+\alpha R(i))$ \\
$(14)$ & in which: $\begin{aligned} R(i) & =e^{-\beta\left(t_{i}-t_{i-1}\right)}(1+R(i-1)) \forall i \geq 2 \\
R(1) & =0\end{aligned}$ \\
\end{tabular}

\section{Multivariate Hawkes process}

In its multivariate version, the Hawkes point process allows to model inter-events arrival dependency. In addition to be potentially affected by the occurrence of events of the same type, arrival of events of a given type can be affected by the occurrence of events of different types. Therefore, in addition to the self-excitation phenomenon described previously, multivariate Hawkes processes allow for mutual-excitation. As described by Embrechts, Liniger et al. (2011), assuming $M \in \mathbb{N}$, a mutually-exciting Hawkes process counting events taking place at times $\left\{\left\{t_{i}^{m}\right\}, m=1, \ldots, M, i=1, \ldots, N^{m}(t)\right\}$ is defined as $\left\{N^{m}(t), m=1, \ldots, M, N^{m}(t) \in \mathbb{N}\right\}$. As for the univariate case, we focus on the simple exponential kernel version of the mutually exciting 
Hawkes process with constant baseline intensity, which is characterized by the following discretized intensity functions:

(15) $\quad \lambda^{m}(t)=\mu^{m}+\sum_{n=1}^{M} \sum_{t_{k}^{n}<t} \alpha^{n / m} e^{-\beta^{n / m}\left(t-t_{k}^{n}\right)}$

where

$\lambda^{m}(t):$ component $m$ process intensity

$\mu^{m} \geq 0$ : component $m$ intensity baseline

$t_{k}^{n}$ : time of the $k^{t h}$ occurrence of event type $n$

In this expression, $m$ corresponds to the type of explained event and $n=1, \ldots, M$, to those of explanatory events. We refer to $n$ and $m$ as types for the predecessor and successor events. We introduce the $n / m$ notation to designate an events relationship, or relationship, in which event type $n$ act as the processor and event type $m$ as the successor. In such case, we claim event of type $n$ occurrences to have an effect on the occurrence of events of type $m$. In this context, $\alpha^{n / m}$ represents the immediate effect of an event of type $n$ occurrence on type $m$ event intensity. Similarly, $\beta^{n / m}$ corresponds to its exponential decay factor. It determines how the impact of past type $n$ events persists in the intensity of type $m$ event over time. It is important to note that while $m \neq n$ represents mutually-exciting relationships, when present, the $m=n$ case relates to a selfexciting relationship. Also, despite the fact that mutual-excitation is supported by the multivariate Hawkes process, dependency structure symmetry is not mandatory. Indeed, assuming $m \neq n$, the presence of a $n / m$ relationship does not involve that of an $m / n$ relationship.

As useful as in the univariate context, the following expression defines the multivariate Hawkes process compensator:

\begin{tabular}{|r|r|}
\hline$(16)$ & $\Lambda^{m}(s, u)=\mu^{m}(u-s)+\sum_{n=1}^{M} \sum_{t_{k}^{n}<\mathrm{s}} \frac{\alpha^{n / m}}{\beta^{n / m}}\left[e^{-\beta^{n / m}\left(s-t_{k}^{n}\right)}-e^{-\beta^{n / m}\left(u-t_{k}^{n}\right)}\right]$ \\
& $+\sum_{n=1}^{M} \sum_{s \leq t_{k}^{n}<\mathrm{u}} \frac{\alpha^{n / m}}{\beta^{n / m}}\left[1-e^{-\beta^{n / m}\left(u-t_{k}^{n}\right)}\right]$
\end{tabular}


In the spirit of (8), the branching ratio $B R^{n / m}=\alpha^{n / m} / \beta^{n / m}$ is now interpreted as the expected number of type $m$ events related to the occurrence of an event of type $n$.

As described by Toke (2011), a branching ratios matrix $\Gamma=\left(\alpha^{n / m} / \beta^{n / m}\right)_{m, n=1, \ldots, M}$ spectral radius strictly smaller than one represents a sufficient condition for a Hawkes process stationarity. ${ }^{3}$ Afterward, assuming a process stationarity, the unconditional expectation of its components intensity is given by (17), in which $\lambda$ corresponds to the $E\left[\lambda^{m}(t)\right]_{m=1, \ldots, M}$ vector, and $\boldsymbol{\mu}$ to the baseline intensities $\left(\mu^{m}\right)_{m=1, \ldots, M}$ vector.

$$
\lambda=(\boldsymbol{I}-\boldsymbol{\Gamma})^{-1} \boldsymbol{\mu}
$$

The log-likelihood function of a multivariate Hawkes process can be represented as the sum of its components log-likelihood functions. This interesting feature for speed and computational intensity considerations is possible because of the absence of shared parameters between the components. The general process log-likelihood is thus provided by the following expression:

\begin{tabular}{|c|c|}
\hline$(18)$ & $\ln \mathcal{L}\left(\left\{t_{i}^{m}\right\}_{m=1, \ldots, M, i=1, \ldots, N^{m}}(T)\right)=\sum_{m=1}^{M} \ln \mathcal{L}^{m}\left(\left\{t_{i}^{m}\right\}_{i=1, \ldots, N^{m}(T)}\right)$ \\
\hline
\end{tabular}

The log-likelihood function of a single component of the multivariate Hawkes process is provided by the following expression:

\begin{tabular}{r|}
$\ln \mathcal{L}^{m}\left(\left\{t_{i}^{m}\right\}_{i=1, \ldots, N^{m}(T)} \mid\left\{t_{k}^{n}\right\}_{n=1, \ldots, M, k=1, \ldots, N^{n}(T)}\right)$ \\
$=-\mu^{m} T-\sum_{n=1}^{M} \sum_{k=1}^{N^{n}(T)} \frac{\alpha^{n / m}}{\beta^{n / m}}\left[1-e^{\left.-\beta^{n / m}\left(T-t_{k}^{n}\right)\right]}\right.$ \\
\\
$+\sum_{i=1}^{N^{m}(T)} \ln \left(\mu^{m}+\sum_{n=1}^{M} \sum_{t_{k}^{n}<t_{i}^{m}} \alpha^{n / m} e^{-\beta^{n / m}\left(t_{i}^{m}-t_{k}^{n}\right)}\right)$
\end{tabular}

\footnotetext{
${ }^{3}$ The spectral radius of the matrix $\boldsymbol{A}$ is defined as $(\boldsymbol{A})=\max _{a \in S(\boldsymbol{A})}|a|$, where $S(\boldsymbol{A})$ corresponds to the set of all eigenvalues of $\boldsymbol{A}$.
} 
Finally, in the way (14) did before, (20) provides a more computationally efficient version of this function.

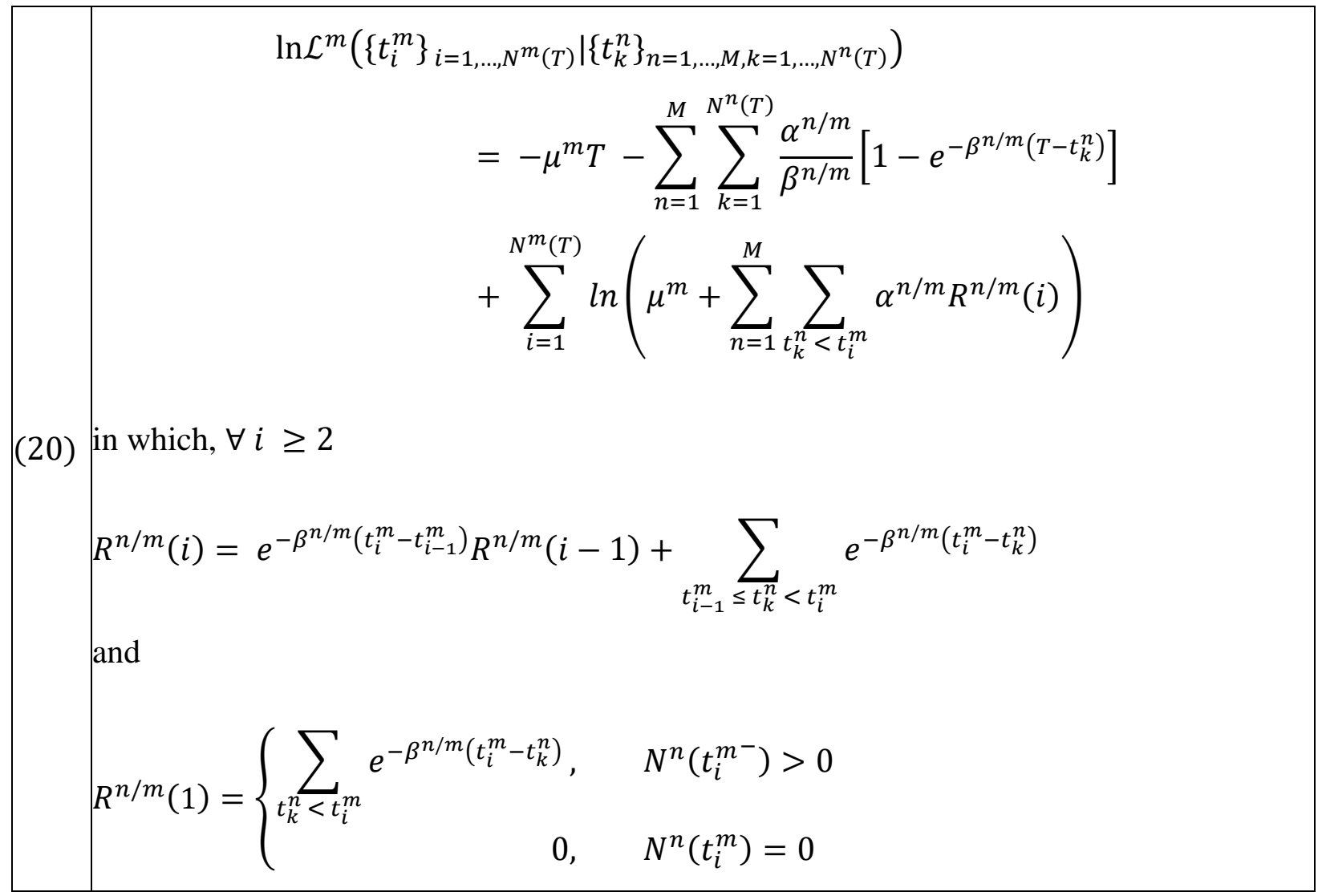

\section{The data}

As introduced before, this paper empirical analysis is performed using an extensive three months Xetra dataset. Given the important number of parameters that may result from our models selection and estimation methodology, we restrict our analysis to the stocks of three individual companies: BMW, SAP, and Adidas. This restriction increases our high-dimensional result sets intelligibility. BMW is an automobiles and motorcycles manufacturer, SAP an enterprise software corporation and Adidas (ADS), an important player of the shoes, clothing, and accessories industry. These companies stocks are DAX index components, which encompasses the 30 main German blue chips trading on the Frankfurt Stock Exchange.

The time period covered in this paper spans from February $1^{\text {st }}$ to March $31^{\text {st }}, 2013$. It encompasses 61 trading days over which BMW, SAP and ADS stand in the DAX Index second tier in terms of 
the total traded volume. These liquid stocks respectively occupy $16^{\text {th, }} 12^{\text {th }}$ and $19^{\text {th }}$ ranks with approximately 115, 187 and 58 million of traded shares. These ranks slightly improve when it comes to the total traded amount. Regarding this second metric, our stocks occupy the $11^{\text {th }}, 7^{\text {th }}$ and $14^{\text {th }}$ positions with rough totals of 8, 11.4 and 4.4 billion EUR. Similar to traded shares, although close in ranking, SAP exchanged amount is $42.5 \%$ larger than BMW that already represents an 81.8\% improvement over ADS. This documents the fact that when it comes to traded volumes, the DAX index components show important discrepancies. As an example, during our reference period, the average exchanged amount of stocks constituting the most traded half of the DAX index in these terms appears 3.3 times larger than for those included in the other half. Therefore, we claim that selecting our three stocks inside the second tier of the DAX index in terms of traded volumes ensures diversity even if they seem closely related at some levels. From this point, to simplify the reading of information related to our three liquid stocks, we use a vector inspired notation in which [BMW; SAP; ADS] reports the values specific to BMW, SAP, and ADS.

A DAX stock normal trading day essentially consists in five steps. The opening auction takes place between 8:50 and 9:00. ${ }^{4}$ It is followed by a four-hour continuous trading session that is suspended at 13:00, leaving the room for the intraday auction that last for a minimum of 2 minutes. Afterward, the market resumes for a four and a half hour continuous trading session ending at 17:30, which corresponds to the closing auction start time. This last auction period ends between 17:35:00 and 17:35:30. In order to focus on the most typical intraday market conditions, we discard the post opening auction as long as the pre and post intraday auction 30 minutes continuous trading periods. As a result of these exclusions, our trading day consists in both a morning and an afternoon period going from 9:30 to $12: 30$ and 13:30 to 17:30, which sums to 7 trading hours.

Although transformed, our dataset originates from Xetra Enhanced Broadcast Solution 13.0, a piece of software responsible for transmitting real-time market information to Frankfurt Stock Exchange participants. In addition to executed transactions information, it includes microsecond timestamped update information for 20 price levels on both sides of the Limit Order Book (LOB). Regarding our 61 seven-hour trading periods, BMW, SAP, and ADS approximately count 114, 106 and 72 million of these LOB updates. It is interesting to notice that although less traded in

\footnotetext{
${ }^{4}$ Opening, intraday and closing auction sessions have a random ending time occurring during the 30 second period following 9:00:00, 13:02:00 and 17:35:00.
} 
terms of shares and euros volumes, BMW is slightly more active than SAP from a LOB perspective. An update is reported for any change occurring on the first 20 price levels of both sides of the LOB. They are generally related to trade executions, limit order submissions, and limit order cancellations. It is possible for multiple price levels variations to be reported through the same update. Figure 1 presents the empirical cumulative distribution functions of the durations between reported LOB updates. With the exceptions of the proportion of changes being reported in less than one microsecond that appears to be more important for BMW and those with an interval between 10 and 160ms that seems slightly higher for SAP, this figure shows that our stocks present similar characteristics in terms of LOB update durations. More than $75 \%$ of these variations are separated by less than $100 \mathrm{~ms}$ and about $95 \%$ of them by less than one second. These large proportions of close LOB changes are consistent with the presence of high frequency trading activities.

\section{The events}

Our first step in describing a multi-level order book events arrival dynamics consists in identifying the events of interest. At the highest level, market participants interact through orders submission and cancellation. We consider each of these individual actions as an event occurrence that must be classified based on its effect on the LOB. As long as their impact on available liquidity, best available prices and bid-ask spread, we use the affected LOB side and depth level as events classification drivers. From our financial point of view, we also take the investor intention behind each event type into consideration. As an example, assuming they involve the same number of shares, a trade execution and a limit order cancellation may have the same effect on the best available price and available liquidity. However, the intentions behind the decisions leading to the new order book state being clearly different, we consider important to make a distinction between these two types of events. Widely inspired by Large (2007), we divide the events into four global categories : Trades with best price impact (Trades w/ BPI), Trades without best price impact (Trades w/o BPI), Limit order submissions with best price impact (LOS w/ BPI) and LOB events. We divide this last category into limit order submissions taking place on the current best price depth level and beyond (LOS), and Limit order cancellations (LOC).

An actual share exchange taking place on an order-driven stock market generally results from the submission of a market order or an aggressive limit order. We define this second element as a bid 
(ask) limit order for which the price is equal or higher (lower) than the best available ask (bid) price, which lead to its immediate partial or total execution. Our dataset providing no explicit information on these transactions starting points, depending on the affected book side, we simply refer to these events as buy and sell trades. However, in our attempt to capture the largest possible number of dependency effects, we follow Large (2007) and make the distinction between trades affecting the best available price and the bid-ask spread from those who do not. In our Xetra context, we relate these categories to transactions entirely consuming the first depth level and beyond, or trades-through (see Toke and Pomponio (2012)), and those partially affecting this best price level. We denote Trades w/ BPI as Buy* (Sell*) events and Trades w/o BPI as Buy (Sell). Panel I of Figure 2 visually presents a Buy* trade event example. The best ask price level being entirely consumed, while the bid-ask spread increases from 0.02 to 0.03 , the pre-trade second best ask price becomes the post-trade best ask price. Similarly, Panel II shows the effects of a Buy event. In this case, the best ask level is partially consumed with no effect on the best ask price and the bid-ask spread.

We define a distinct event categories for LOS and LOC events taking place on the bid and ask sides of the book on a depth level basis. As with trade events, we make the distinction between LOS with and without best price impact. While referring to bid and ask LOS w/o BPI events as BA1 and AA1, we identify bid and ask LOS w/ BPI as BA1* and AA1*. Representing the arrival of a new limit order inside the bid-ask spread, these last events present some particularities. First, for these events to be feasible, the spread must be wide enough to allow the creation of a new price level. Consequently, in situations where the bid-ask spread is only one tick wide, it is impossible for $\mathrm{BA} 1 *$ and $\mathrm{AA} 1^{*}$ events to be observed. Because of this specificity, Zheng et al. (2014) have modeled these events arrival using constrained Hawkes processes. However, our BMW, SAP and ADS stocks presenting a bid-ask spread wider than one tick for 92, 70 and 88 percent of the complete 61 trading days dataset, we keep relying on the traditional multivariate Hawkes processes for the representation of each of our events arrival sequences. Second, assuming the creation of a price level inside a favorable spread, the only event occurrence identified as BA1* or AA $1 *$ is the one related to the submission of this new level first order. As long as its price remains the best available, depending on its book side, any LOS taking place on this depth level will be identified as a BA1 or AA1 event. Panel III of Figure 2 provides a simple ask LOS w/ BPI event example. As for the previous examples, before the event occurrence, the best available ask price is 1.06 with 
a 0.02 bid-ask spread. Then, an ask 1.05 limit order is submitted, leading to the creation of a new best ask price level and a decreased bid-ask spread that goes from 0.02 to 0.01 , which situation is identified as the occurrence of an AA1* event.

Our extension of Large (2007) events classification mostly concerns limit order submissions without best price effect and limit order cancellations. In this paper, the author defines four event types, one for each combination of book side (bid or ask), and LO action (submissions or cancellations). In order to include a depth dimension into our LOB events arrival analysis, in addition to the book side, we group LOS and LOC events on the basis of the depth level number, which is relative to the best bid or ask price on which they take place. Disregarding the involved prices, we refer to LOS w/o BPI events taking place on the twenty referenced depth levels as BA1 to BA20 for the bid book side, and AA1 to AA20 for the ask side. Similarly, we denote bid and ask $\mathrm{LOC}$ events as $\mathrm{BC} 1$ to $\mathrm{BC} 20$ and $\mathrm{AC} 1$ to $\mathrm{AC} 20$. This is to reduce the number of categories required to cover the same price range that we choose this best prices relative approach rather than that of Cont et al. (2010) who uses prices tick grids in order to define their events arrival processes. Because of this choice, we identify three possible scenarios leading to the identification of the same LOS event type. Figure 2 provides an example for each of them where the type of the identified event is AA2. First, Panel IV presents the trivial case where a limit order is submitted on the existing second best ask price level, increasing its available liquidity. Second, in the Panel VI example, no liquidity with a 1.07 price is available in the pre-event LOB. Therefore, resulting from a limit order submission leading to the creation of a new depth level on this available tick space, the event is identified as taking place on the second best price level, despite the pre-event prevalence of a different depth level 2. With the same idea, Panel VI presents a situation where a submitted limit order also leads to the identification of a depth level 2 LOS event despite the fact that room is available for a 1.07 new second best price level. In this case, the post-event 1.08 involved price level still corresponds to the second best price. Closing our events identification examples, Panel VII finally presents a BC1 event case in which the liquidity available on the bid best price level is decreased by the size of the cancelled order.

Having formally identified the events of interest, Figure 3 presents daily average number of the events studied in this paper for our three liquid stocks. Globally, Panels (a) to (c) suggests limit order submissions to be more frequent than transactions. Disregarding their detailed classification, 
for each trade event, our liquid stocks are subject to averages 30.5, 26.9 and 28.2 limit order submissions. When it comes to the most aggressive events, Panel (a) shows Trades w/ BPI to be slightly more frequent than Trades w/o BPI. The actual number of shares consumed in these trades being not considered in our classification, it is not surprising to observe an almost equal numbers of Trades w/ BPI and Trades w/o BPI events. Although likely for very large trades to consume more than one price level, these events classification remains dependent of the LOB shape. For the same number of consumed shares, it is possible for a trade to be classified as either Buy* (Sell*) or Buy (Sell), depending on the pre-trade LOB. As we decrease on the events underlying action aggressively scale, Panels (b) and (c) suggest that while more common than Trades, BA1* and $\mathrm{AA} 1 *$ appear less frequent than BA1 and AA1. We relate this observation to the fact that LOS w/o BPI events are not restricted by the previously described bid-ask spread conditions and involve less commitment than LOS w/ BPI underlying actions. Finally, regarding deeper LOB events, Panels (b) to (d) show that for LOS as well as LOC, an important part of the action actually take place on depth levels 1 to 5. Beyond this point, we denote a decrease in events arrival up to depth level 10 for LOS and level 11 for LOC where some local peaks are observed. Afterward, the number of occurrences appears generally constant up to level 17. Finishing with depth levels 18 to 20, although LOC events appear slightly more common than LOC, a very small number of both event types appear to take place this far from the best bid and ask.

\section{The model}

In this section, we relate Hawkes processes theory to our events definition through the definition of our potential arrival processes. We begin by defining the set of all events using the following expression:

(21) $\quad S=\left\{\begin{array}{c}B u y^{*}, S_{e l l}^{*}, B u y, \text { Sell, } B A 1^{*}, A A 1^{*}, \\ B A 1, \ldots, B A 20, A A 1, \ldots, A A 20, B C 1, \ldots, B C 20, A C 1, \ldots, A C 20\end{array}\right\}$.

In addition to Trades w/ BPI, Trades w/o BPI and LOS w/ BPI, this set includes LOS w/o BPI and LOC events taking place on depth levels 1 to 20 for both sides of the book, which represents a total of 86 event types. Obtained from equation (15), the next expression represents the intensity function of any event $m \in S$ process on a given trading day $d=1, \ldots, 61$. 


$$
\lambda_{d}^{m}(t)=\mu_{d}^{m}+\sum_{n \in S^{m}} \sum_{t_{k}^{n}<t} \alpha_{d}^{n / m} e^{-\beta_{d}^{n / m}\left(t-t_{k}^{n}\right)}
$$

In this definition, $S^{m}$ corresponds to the set of predecessor events used to explain the arrival of the successor event $m$. In a complete model context, $\forall a \in S, S^{a}=S$, which is related to the previous section notation by $|S|=M$. However, $S$ representing 86 event types, a complete model would involve 7396 events relationships, which would lead to the estimation of 14878 parameters on a daily basis. We establish rules to limit the number of explanatory events involved in some of the intensity processes. Based on events categories and affected depth levels, we define four sets of explanatory events acting as starting points in the estimation methodology presented in the next section. These sets definition are presented in Table 1. Given a successor event $m \in S$ (column), $S^{m}$ includes the checked explanatory events groups (rows). First, Trades and LOS w/ BPI arrival intensity processes are initially unrestricted. Indeed, taking advantage of the data availability, we test these successor events arrival for all potential dependency relationships. Second, Trades and LOS w/ BPI are part of the potential explanatory events set of all LOB events. Then, we define the exact set of each of them on the basis of its occurrence depth level. Following an incremental pattern, the potential explanatory events sets for LOS and LOC events taking place on depth levels 1 to 3, 4 to 6 and 7 to 10 respectively include levels 1 to 3,1 to 6 and 1 to $10 \mathrm{LOB}$ events. In a last increment, no restriction are imposed on depth levels 11 to 20 LOB events arrival intensity processes. By imposing these restrictions, the number of relationships potentially included in our daily models is reduced to 5268, which leads to 10622 estimated parameters. We consider this $29 \%$ reduction as an interesting trade-off between model exhaustivity and estimation efficiency.

\section{Estimation methodology}

Having defined an extensive set of potentially related events and imposed initial restrictions to our multivariate Hawkes process, we intent to identify the most recurrent dependency relationships observable for our three stocks over our data sample period. Each trading session being unique in many ways, we do not attempt to analyze each daily idiosyncrasy present in these stocks events dependencies structures. However, by focussing on the significant and persistent relationships, we expect to identify and depict some of their more general characteristics. In order to identify these interrelations, we develop a two part estimation methodology that we apply on each of our three 
liquid stocks. First, on a trading day basis, we identify the members of the previously defined sets of potential explanatory events that have a significant effect on the arrival of their respective successor event. We refer to the daily Hawkes processes made of these relationships as complete models. Then, selecting the most recurrent relationships from these daily models, we define a final Hawkes process that we identify as a descriptive model. Afterward, it becomes possible to perform our analysis using the daily parameters estimated value of the three descriptive models. It is important to note that despite the fact that the initial sets of potential explanatory events are the same for each stock, since they do not present the same recurrent events relationships, we observe discrepancies across the components of the three descriptive models.

Estimating high-dimensional Hawkes processes parameters presents some challenges. The loglikelihood function being not strictly concave, it is possible for the numerical maximization procedure to find a local maximum instead of a global one. ${ }^{5}$ Our experiments suggest this method to offer a better performance in situations where the underlying process is not crowed by multiple non-significant relationship parameters to estimate. Parameters initial value selection also appears to represent a key factor to convergence. Having these issues in mind, we establish that in our high-dimensional context, for a given successor event, estimating the parameters of a Hawkes process simultaneously by initially including all the concerned predecessor events may not represents the best avenue. Indeed, our main objective being to identify relevant predecessor / successor events relations without prior assumption, testing all of them in a single pass may represents an attempt to estimate multiple non-significant parameters. In the same spirit, providing appropriate initial values for all the estimated parameters may become a challenging task. In this context, we use an iterative methodology allowing to divide the whole parameters estimation task into smaller sub-tasks. By successively introducing potential relationships into the model, it becomes possible to discard the less relevant ones early in the process.

Working on a stock, trading day and dependant event basis, our methodology first step consists in estimating the parameters of a simple univariate Hawkes model for each potential explanatory event. Each of these models assumes the included predecessor event type as the only one involved

\footnotetext{
${ }^{5}$ We perform parameters estimation using Matlab interior-point algorithm. In situations where this algorithm does not converge correctly, the BOBYQA algorithm implemented in the free NLOpt package is used for a second estimation pass. In the rare cases for which this second algorithm also fails to converge, the tested dependency relationship is discarded.
} 
in the successor event intensity process. The main objective of this first step is to obtain parameters initial values to be used in the next estimation round in which candidate explanatory events are successively introduced into the model. It also allows us to go through first relationships discarding round. Explanatory events for which the estimated $\alpha$ parameter $\mathrm{p}$-value is larger than 0.01 are immediately excluded. These values are obtained by inverting the Hessian matrix at the loglikelihood maximum point identified by the non-linear optimization algorithm. We also exclude events relationships for which the $\alpha$ parameter value, although appearing significant, is smaller than 0.1. Finally, we discard any relationship presenting a branching ratio (BR) smaller than 0.02. In this case, we consider the predecessor event to have a neglectable effect on the successor event arrival. By performing this first discarding task early, we attempt to ease the next estimation rounds that may benefit from reduced sets of potential explanatory events.

The second step represents the core of our estimation methodology. It generally consists in multiple model estimations for each explained event type. For each of them, we begin with the set of predecessor events that have not been discarded from the previous step. We build the foundations of each daily complete model iteratively. On each iteration, a new explanatory event candidate is introduced into the evolving model. We use parameters value obtained in the first step as optimization algorithm initial values for the newly introduced predecessor event. Once the model parameters are estimated, events relationships appearing non-significant or weak are discarded. We keep using the criteria described in the first step to perform these exclusions. The non-discarded explanatory events remain in the model for the next iteration. In these cases, rather than using step one coefficients as initial values for the optimization algorithm, we use their last estimated values. This iterative procedure goes on until all step one predecessor events candidates have been tested in the model. At this stage, we identify the Hawkes process model containing the non-discarded events as the complete model for the trading day.

For each stock, the last step of our methodology consists in building a descriptive model and estimating its parameters value for each trading day. We simply use the number of days for which event relationships have been confirmed as a part of a daily complete model to select the components of these final models. We choose each predecessor event considered as having a significant effect on the successor event on at least 31 days. This threshold ensures the relationships included in our final models to have been part of more than 50 percent of the daily complete 
models. Once this task is completed, each model parameters are estimated on a daily basis. These estimated values that represent a source of events dependency information are used to perform our next section analysis.

\section{The results}

\section{Descriptive models selection}

Reporting results from the first step of our estimation methodology, Table 2 presents the number of trading days for which events relationships meet our selection criteria. Despite relying on preliminary observations, we consider this table as a general events arrival dependency map. The different panels split explained events types by categories and depth levels of occurrence. Each cell represents a potential relationship for which the row and the column link a predecessor event to a successor event. We use the number of trading days over which the selection criteria are met to classify these relationships. First, we qualify events effects encountering the selection conditions on 0 to 6 trading days (out of 61) as absent. Conversely, we define those for which the numbers of days lies between 55 and 61 as persistent. While fairly straightforward to categorize these first relationships groups, the task increases in ambiguity when it comes to those presenting a number of effective trading days lying between these two opposite ranges bounds. In this context, we define events effects meeting our significance criteria on 7 to 30 trading days as sparse. We relate these effects to algorithms and trading mechanisms that have a significant impact on a limited number of trading days. On the other hand, because of their impact on the main part of our analysed period, we classify effects observed on 31 to 54 days as frequent. Therefore, having established our selection threshold to $50 \%$ of the trading days, these last effects combine to those identified as persistent to form our descriptive models dependency components.

Table 2 Panel I first shows that for our liquid stocks, Trades w/ BPI and Trades w/o BPI events appear only affected by Trades w/o BPI and LOS w/ BPI on a recurrent enough basis to be included in our descriptive models. As mentioned before, we have defined our initial candidate models in order to be able to take advantage of the data availability and analyse the effects of LOB events on the arrival of Trades w/ BPI and Trades w/o BPI events. However, having performed an extensive search for the presence of BA1 to BA20, AA1 to AA20, BC1 to BC20 and AC1 to AC20 effects on the arrival of Buy*, Sell*, Buy and Sell events, we conclude in the general absence of such 
relationships. As presented in Panel I, despite some sparse exceptions, most of these relationships have met our selection criteria on less than 7 trading days, which are qualified as absent based on our previously defined scale. We relate this lack of recurrent effects to the proportion of LOB events relative to Trades. In line with the previously presented numbers, regardless of the depth level on which they take place, disregarding LOS w/ BPI, the average number of LOB events appears 50.8 to 57.3 times higher than the number of Trade events. This information suggests the LOB to be the theatre of multiple LOS and LOC strategies that, most of the time, do not lead to actual shares exchange. Therefore, we consider consistent that the Trades events arrival do not appear systematically affected by single events arising from these extensive games. On the other hand, the presence or absence of Trades w/o BPI and LOS w/o BPI effects on Trades w/ BPI and Trades w/o BPI events seems related to the LOB side affected by both predecessor and successor events. Indeed, Panel I shows that while Buy/Buy*, Sell/Sell*, Buy/Buy and Sell/Sell appear frequent to persistent, it is possible to qualify Buy/Sell*, Sell/Buy*, Buy/Sell and Sell/Buy as absent. Then, presenting lower contrast levels, AA $1 * / B u y *$ and BA1*/Sell* appear to be highly persistent for our three stocks while BA1*/Buy* and AA $1 * /$ Sell* span from sparse for SAP, to frequent in the cases of BMW and ADS. Consequently, the SAP descriptive model imposes restrictions on these two last relationships, which is not the case for BMW and ADS. We use the same guidelines for $\mathrm{AA} 1 * / \mathrm{Buy}$ and $\mathrm{BA} 1 * / \mathrm{Sell}$ that appear frequent in the BMW and SAP cases but meet our selection criteria on less than half the trading days for ADS.

Panel I of Table 2 also indicates that LOS w/ BPI (BA1* and AA1*) arrival seems mainly affected by Trades and other $\mathrm{BA} 1 *$ and $\mathrm{AA} 1 *$ events. With some rare sparse exceptions, these events appear generally unaffected by LOS and LOC occurring on depth levels 2 to 20. The situation differs when it comes to LOS and LOC events taking place on the prevalent best price levels. We observe some BA1, AA1, BC1 and AC1 events effects on the arrival processes of BA1* and AA1*. The actual presence or absence of such relationships appear directly related to the involved events book side of occurrence. Indeed, in a radical contrast with the high BA1/BA1* and AA1/AA1* persistence levels, we note a complete absence of BA1/AA1* and AA1/BA1* relationships. Similarly, while $\mathrm{BC} 1 / \mathrm{AA} 1 *$ and $\mathrm{AC} 1 / \mathrm{BA} 1 *$ appear highly persistent, we observe various levels of recurrence for $\mathrm{BC} 1 / \mathrm{BA} 1 *$ and $\mathrm{AC} 1 / \mathrm{AA} 1 *$ that go from sparse to persistent. In this context, since it meets our criteria on an insufficient number of trading days, AC1/AA1* is excluded from our BMW descriptive model. Trades w/o BPI effects on LOS w/ BPI also appear related to each 
event book side. Indeed, while Buy/BA1* and Sell/AA1* show an important level of persistence, Sel1/BA1* and Buy/AA1* appear almost inexistent. Finally, the persistent Buy*/BA1* and Sell*/AA1* and frequent to persistent Sell*/BA1* and Buy*/AA1* relationships preliminarily suggest LOS w/ BPI events occurrence probability to be increased by the two types of Trades w/ BPI events.

Representing the last elements of this preliminary results overview, Panels II to IX of Table 2 present relationships involving LOS w/o BPI and LOC events as the successor counterpart. Since most of these results appear symmetrical for both sides of the book, we focus on dependent events taking place on the bid side of the book, which correspond to Panels II to V. These panels show that the recurrence level of some relationships sharing predecessor and successor events category may appear related to the depth levels over which these involved events take place. This translates into dependency zones delimited by predecessor and successor events depth level of occurrence over which, relationships may present increasing, decreasing, or similar levels of recurrence.

As shown in Panels II to V, the number of event types affecting LOS w/o BPI and LOC arrival recurrently enough to be included in our descriptive models appears non monotonically decreasing as the depth level on which these successor events take place increases. For our liquid stocks, while presenting a peak regarding $\mathrm{BA} 1$ to $\mathrm{BA} 4$ and $\mathrm{BC} 1$ to $\mathrm{BC} 4$, the trend becomes generally decreasing when it comes to LOB events occurring on depth levels 5 to 12 . Beyond this point, when greater than zero, the number of event types having a recurrent effect on the arrival of LOS w/o BPI and LOC is limited to one or two. Panels III and V show that depending on their category, successor events appear recurrently affected by the arrival of at least one predecessor event type up to a depth level rank lying between 16 and 19. BA11 to BA20 and BC11 to BC20 events arrival processes appear generally unaffected by other LOS w/o BPI and LOC occurrence, no matter the depth level on which these predecessor events take place. Still shown in Panels III and V, with three frequent and one persistent exceptions regarding LOS w/o BPI, the 1600 relationships of these natures that have been processed through our estimation methodology appear at most sparse. We observe important dependency zones over which relationships have not met our selection criteria on a single trading day. 
Panels II to V also suggest LOS w/o BPI and LOS arrival to be generally affected by Trades and LOS w/ BPI events. We observe some of these events to have a recurrent effect on LOS w/o BPI arrival up to level 16 and LOC up to level 19. With rare exceptions, they remain the only events identified by our methodology as having a recurrent effect on LOB events taking place above level 11. Leaving the detailed dynamics description to a subsequent section, we observe the absence, presence, and recurrence of these relationships to appear highly related to the LOB side affected by the involved events. Moreover, although presenting different patterns, the number of trading days over which relationships meet our selection criteria appear decreasing as we move higher in the depth levels. Taking the example of Trades w/ BIP / LOS w/o BPI, despite some minor discrepancies among our liquid stocks, while Buy* / BA1 to BA3 appear generally persistent, we note the Buy* effects recurrence level to go from frequent to sparse in the BA4 to BA7 successor events zone. Past this point, with some sparse exceptions, it becomes absent in the BA9 to BA20 area. On the other hand, Sell* events present a generally frequent to highly persistent effect on BA1 to BA16.

\section{Data fitting}

Having selected a complete descriptive model for each of our three liquid stocks, the second step of our methodology involves estimating their parameters on a trading day basis. In this section, we use the resulting set of estimated values to perform general data fitting analysis. Our models essentially serving descriptive purposes, we do not consider this paper in the best data fitting race. Indeed, we consider the basic exponential kernel appropriate for the achievement of our general events relationships analysis task. That being said, it remains obvious that each of our model should deliver a minimum data fitting performance to be considered as serious candidates. Fortunately, we demonstrate that various segments of our descriptive models do not only perform well when compared to the basic Poisson homogeneous model, they also offer satisfying performances in absolute terms. Widely used for point processes data fitting analysis, Figure 4 presents a quantile-quantile plot (Q-Q plot) for each stock and explained event type pair. Since a perfect fitting would involve inter-event compensators to follow a unit mean and standard deviation exponential distribution, each graphic presents this theoretical distribution as the dotted diagonal line against which our descriptive model inter-event compensators are plotted on a quantile basis. For comparison purposes, we also present the quantiles obtained from 
homogeneous Poisson processes. For each model, inter-event compensators are computed using the corresponding trading day estimated parameters.

In order to investigate the performance gap between our descriptive models and the homogeneous Poisson models, we follow Rambaldi et al. (2017) and introduce the adjusted baseline. In our context, this measure relates to the proportion of an event arrival process that may be related to its constant baseline component, which correspond to $\mu^{m}$ in equation (15). Having estimated expression (22) parameters on a daily basis, for each explained event $m \in S$, we refer to $\hat{\mu}_{d}^{m}$ as the Hawkes intensity process estimated baseline on trading day $d=1, \ldots, 61$. For the same event type and trading day, $E\left[\lambda_{d}^{m}(t)\right]$ reports the arrival process unconditional intensity expectation as defined in equation (17). Therefore, we define the adjusted baseline as $\hat{\mu}_{d}^{m} / E\left[\lambda_{d}^{m}(t)\right]$, which, as introduced before, reports the proportion of trading day $d$ event $m$ intensity attributable to its constant baseline. Hence, in the absence of Hawkes effect in a descriptive model event arrival processes that would correspond to a Poisson process, this measure would take the constant value one. For each event type, Table 3 reports $\overline{\hat{\mu}}_{d}^{m}$ and $\overline{\hat{\mu}_{d}^{m} / E\left[\lambda_{d}^{m}(t)\right]}$, the baseline and adjusted baseline daily average. Figure 5 complements this information with a visual presentation of most LOB events adjusted baseline daily average.

Beginning with Trade w/ and w/o BPI events, Figure 4 Panel I Q-Q plots indicate that despite a performance appearing superior to that of homogeneous Poisson models, this segment of our descriptive models present some weaknesses regarding data fitting. Lu and Abergel (2018) results suggest that these specific events arrival processes could benefit from a double exponential kernel to improve these performance. However, despite these mitigated results, we still consider the simple exponential kernel adequate in our global analysis context. Given our methodology, nothing indicates that the identification of the presence or absence of relationships affecting the arrival of Buy*, Sell*, Buy and Sell events would be affected by the use of a more advanced kernel to describe their intensity processes. Moreover, it is essential to note that despite the fact that a Hawkes process model is considered as a whole, during the estimation step, each events arrival process modeling remains independent of that of the others. Indeed, this representation independence is enforced by the fact that explanatory events occurrence times are exogenous to each dependent event intensity processes. Consequently, in our descriptive context, Trades w/ and w/o BPI imperfect data fitting have no effect on the other 82 LOS and LOC successor event types 
present in our global framework, even if these former predecessor events appear to sometime play important roles in the latter successor events arrival.

When it comes to limit order submission and cancellation events, Figure 4 Q-Q plots presents some interesting trends relative to the data fitting performance of our descriptive models and their Poisson homogenous counterparts. Beginning with LOS w/ BPI, Panel I shows an important performance improvement over Trades. Indeed, despite their previously described particularities, BA $1^{*}$ and AA1* appear adequately represented by our descriptive models. Further into our event types set, Panels II to V show that as we move from the highest to the deepest price levels of occurrence, our models LOS w/o BPI and LOC events arrival processes data fitting performance exhibit some general trends. Despite the facts that these tendencies appear not perfectly monotonic in events depth of occurrence and that each stock presents some idiosyncrasies, by dividing our LOB price levels into three depth segments, it is possible to highlight general patterns that apply to our three liquid stocks. First, our models absolute data fitting performance appears decreasing as we increase in depth level of occurrence. They exhibit their best performance regarding events occurring on low depth levels 1 to 5 with visual results that we consider highly adequate. Beyond this point, our models performance appears to gradually deteriorate. Indeed, Panels II to V show mixed performance regarding events occurring on the order book middle segment consisting in price levels 6 to 11. Despite not catastrophic, events taking place on depth levels 6 to 11 generally exhibit poorer data fitting than what have been observed on lower depth levels. Exceptions are observed for BA10 (AA10) and BC11 (AC11) that present satisfactory results. Afterward, for events taking place on depth levels 12 to 20 , our models data fitting capacity appears generally suboptimal. It is interesting to note that in absolute terms, LOS w/o BPI and LOC events fitting performance present very similar characteristics. From an events arrival dynamics point of view, we consider fortunate that disregarding their types $[72.2 \% ; 78.8 \% ; 66.8 \%]$ of our identified LOB events take place on depth levels 1 to 5 , which correspond to the segment on which our descriptive models present their best performance. On the other hand, we also consider fortunate that only [7.0\%; $4.1 \% ; 8.1 \%$ ] of our identified LOB events appear to occur on depth levels 12 to 20 , which correspond to the LOB segment over which our models shows their least interesting data fitting performance. 
The second tendency relates to our descriptive models performance relative to Poisson homogeneous models. As shown in Figure 4 Q-Q plots the data fitting gap between the two tested frameworks appears to decrease as we increase in LOS w/o BPI and LOC events depth level of occurrence. In addition to being attributable to our selected models deteriorating absolute performance, this trend may be attributed to the improving performance of the Poisson homogeneous models. As before, these tendencies monotonicity being imperfect, they are better perceived when we split the LOB into three depth segments. Back to the low depth levels 1 to 5 events, it is actually possible to note that while our LOS w/o BPI and LOS events descriptive models exhibit their best performance, the Poisson homogeneous models display their poorest results. We relate these visually important gaps to our descriptive models processes low adjusted baseline values. Figure 5 shows that among all events represented, the descriptive models intensity processes of those taking place on the five lowest depth levels present the smallest average adjusted baseline with means of $[0.28 ; 0.21 ; 0.30]$ for LOS w/o BPI and $[0.22 ; 0.23 ; 0.32]$ for LOC events. Such low values indicate a relatively small Poisson homogeneous contribution and large Hawkes effects contribution to their arrival. Indeed, we have to keep in mind that in our current framework, for any event $m \in S$ and trading day $=1, \ldots, 61,1-\hat{\mu}_{d}^{m} / E\left[\lambda_{d}^{m}(t)\right]$ corresponds to the proportion of event $m$ arrival that may be related the Hawkes effects involved in its arrival process. For low levels LOS w/o BPI and LOC events, not only are these effects numerous, but they seems to bring relevant information to the models, which leads to our interesting data fitting results both relative and absolute. Once again, events taking place on the LOB middle segments present mixed results. Regarding these events which take place on depth levels 6 to 11, the decreasing performance gap relative to Poisson homogeneous processes appears mostly attributable to these last framework data fitting improvement. Figure 5 reveals some average adjusted baselines increased values with means of $[0.46 ; 0.48 ; 0.41]$ for LOS w/o BPI and [0.41; 0.49; 0.48] for LOC events. This suggests a tendency for these middle depth events arrival to be slightly more driven by the constant components of our Hawkes processes based descriptive models, which may be related to the reduced visual Q-Q plot gap between them and Poisson homogeneous.

Regarding the events occurring in the deep LOB segment, Figure 5 Q-Q plots show that overall, the quantiles related to our descriptive models events arrival processes are close to those of the Poisson homogeneous processes. We explain this global convergence by the reduced number of events relationships involved in the arrival process of the events taking place in this segment. 
Indeed, as previously presented, these descriptive models processes include a maximum of two Hawkes components. To the extreme, our descriptive models arrival processes for LOS w/o BPI taking place on depth levels 17 to 20 include no Hawkes effects at all, which, as claimed before, leaves us with pure Poisson homogeneous processes. However, unlike events taking place on the two lowest LOB segment, we note an interesting difference between LOS w/o BPI and LOS events in the fact that while quantiles related to the events of the former category almost overlap those of Poisson homogeneous, Panels II to V Q-Q plots show a gap between quantiles related to events of the latter category and those of Poisson homogeneous. Despite subtle, this distinction suggests that on high depth levels, LOC events fitting performance stands out more from Poisson homogeneous than LOS w/o BPI. Leaving the actual events arrival dynamics implications to a subsequent section, we relate this difference to the more important constant baseline participation in LOS w/o BPI than in LOC events arrival processes that is observed in Figure 5. Disregarding the processes including no Hawkes component, this figure shows mean adjusted baseline averages of our descriptive models LOS w/o BPI events arrival of $[0.75 ; 0.72 ; 0.76]$ while LOC events counterpart values equals to $[0.52 ; 0.46 ; 0.50]$, which are closer to the values previously observed for events taking place in the middle depth LOB segments. These numbers suggest a less important constant baseline (more important Hawkes effects) contribution to LOC events arrival processes than to LOS w/o BPI. We consider these elements as a potential explanation for our descriptive models LOC events data fitting performance relative to Poisson that appears to be better than those of LOS w/o BPI when such events take place on depth levels 12 to 20.

\section{Estimated parameters}

Having derived the adjusted baselines from the actual estimated constant baseline parameters, we now focus on Hawkes effects related parameters. On a stock basis, we define events $m \in S$ and $n \in \hat{S}^{m}$, where $\hat{S}^{m} \subseteq S^{m}$ corresponds to the set of explanatory events that have been selected for event $m$ descriptive model intensity process. Back to expression (22), these $m / n$ relationships are daily characterized by the $\alpha_{d}^{n / m}$ and $\beta_{d}^{n / m}$ parameters of which we denote the estimated values as $\hat{\alpha}_{d}^{n / m}$ and $\hat{\beta}_{d}^{n / m}$. Table 4 aggregates these parameters by providing their daily average. Each 2 values cell relates to a selected event $n$ exponential Hawkes effect on an event $m$ arrival. For each $m / n$ effect, $\overline{\hat{\alpha}_{d}^{n / m}}$ relates to the initial intensity increment and $\overline{\hat{\beta}_{d}^{n / m}}$, to the exponential decay 
factor. Although not always speaking for themselves, these values represent the core of our multivariate Hawkes events dependency structure.

Regarding the temporal aspect of our several exponential Hawkes effects, Table 4 shows that for our liquid stocks, $\overline{\hat{\beta}}_{d}^{n / m}$ ranges from [2.70 to $979 ; 1.58$ to $903 ; 11$ to 946]. These values corresponding to exponential decay factors, it may be difficult to appreciate their implications. Therefore, we focus on the more interpretable half-life period (HL) as Hawkes effects duration measure. However, in order to avoid overweighting outlier $H L_{d}^{n / m}$ values in the $n / m$ Hawkes effect duration analysis, instead of computing the actual half-life period daily average $\overline{\ln (2) / \widehat{\beta}_{d}^{n / m}}$, we use $\ln (2) / \widehat{\beta}_{d}^{n / m}$ in which equation (7) is applied on the exponential decay factor daily average. ${ }^{6}$ In this context, Figure 6 presents these values cumulative distribution functions for the $[374 ; 328$; 366] Hawkes effects that have been selected as parts of our descriptive models. With [4.95 ms; $5.91 \mathrm{~ms} ; 5.64 \mathrm{~ms}]$ median values and $[17.4 \mathrm{~ms} ; 27.5 \mathrm{~ms} ; 14.7 \mathrm{~ms}] 90^{\text {th }}$ percentiles, these measures suggests important proportions of individual Hawkes effects to remain effective on very small time spans. Although useful as a general duration indicator, we have to keep in mind that effects subject to exponential decay remain effective past their half-life period. As said before, it actually takes about 6.64 half-life periods for these effects to lose $99 \%$ of their strength. From the previous median half-life periods, it is easy to establish that $50 \%$ of our daily estimated events effects almost totally vanish within [33 ms; $39 \mathrm{~ms} ; 38 \mathrm{~ms}$ ]. Moreover, it is possible to claim that about [99.4\%; 96.2\%; 100\%] of our estimated Hawkes effects dismiss over time periods shorter than $500 \mathrm{~ms}$, which is considered by Moallemi and Sağlam (2013) as a reasonable estimation of the human reaction time. For technical considerations such as the exponential kernel shape and the mutuallyexciting capacity of our Hawkes processes based models, we consider more or less relevant to relate Hawkes effects average effectiveness periods to actual reaction times to events. However, from our point of view, such generalized low average values may suggest algorithm involvement in our three liquid stocks LOB events arrival dynamics.

\footnotetext{
${ }^{6}$ Outlier $H L_{d}^{n / m}$ values result from irregular $\hat{\beta}_{d}^{n / m}$ estimated parameters that may originate from estimation convergence issues or trading days exceptional idiosyncrasy.
} 
When it comes to instantaneous increment in events arrival intensity related to Hawkes effects, Table 4 shows that $\overline{\hat{\alpha}}_{d}^{n / m}$ values range from [0.31 to $170 ; 0.52$ to $181 ; 1.18$ to 153$]$ events per second with $[10.27 ; 9.49 ; 8.9]$ median values. These numbers represent $[1.7$ to $822 ; 1.4$ to $1368 ; 5$ to 1091] times the involved predecessor event unconditional expected intensity $E\left[\lambda_{m}^{d}(t)\right]$ with [74; 58; 96] median values ${ }^{7}$. Like exponential decay factors and effects HL period, although suggesting potentially important instantaneous intensity increases, it is highly difficult to interpret the $\overline{\hat{\alpha}_{d}^{n / m}}$ values by themselves. Indeed, to adequately quantify an actual predecessor event $n$ impact on a successor event $m$ arrival on a trading day $d$, the initial intensity surge has to be placed in its temporal context, which relate to the exponential decay factor. Back to our daily aggregated context, Figure 7 Panel I shows a relation in the magnitudes of $\overline{\hat{\alpha}_{d}^{n / m}}$ and $\overline{\hat{\beta}_{d}^{n / m}}$, which makes comparing Hawkes effects with different effective periods very difficult. Adding the fact that several hundred relationships have been identified, it becomes obvious that an analysis directly based on these two parameters for each effect would be inconvenient. In this context, the branching ratio $(\mathrm{BR})$ becomes an interesting measure of effect strength. As defined before, $B R^{n / m}$ corresponds to the effect of a single event $n$ occurrence on event $m$ compensator. Therefore, it represents the expected number of event $m$ that may be related to the arrival of an event $n$. Back to our daily aggregated framework, Figure 7 Panel II shows that unlike what we have observed for $\overline{\hat{\alpha}_{d}^{n / m}}$, the magnitude of $\overline{B R_{d}^{n / m}}=\overline{\hat{\alpha}_{d}^{n / m} / \hat{\beta}_{d}^{n / m}}$ appears stable in $\overline{\hat{\beta}_{d}^{n / m}}$, which makes our several Hawkes effects comparable when using this metric. Additionally, $B R_{d}^{n / m}$ s.t. $d=1, \ldots, 61$ appears more stable than $\hat{\alpha}_{d}^{n / m}$ and $\hat{\beta}_{d}^{n / m}$. Figure 8 provides visual general examples for these metrics dispersion with respect to their daily average. Panel I presents $[0.29 ; 0.28 ; 0.29]$ and $[0.32 ; 0.28$; $0.27]$ coefficients of variation for $\hat{\alpha}_{d}^{B u y * / B A 1 *}$ and $\hat{\beta}_{d}^{B u y * / B A 1 *}$. On the other hand, the $B R_{d}^{B u y * / B A 1 *}$ coefficients of variation are $[0.16 ; 0.16 ; 0.15]$, which is $[1.8 ; 1.7 ; 1.9]$ and $[1.9 ; 1.7 ; 1.8]$ times smaller than those observed for the previous two estimated parameters. ${ }^{8}$ Such information suggests

\footnotetext{
${ }^{7}$ We exclude BA1*/BC11 and AA1*/AA11 relationships that we identify as outliers with $\overline{\hat{\alpha}_{d}^{B A 1 * / B C 11}}$ values of [362; 322; 324] and $\overline{\hat{\alpha}_{d}^{A A 1 * / A C 11}}$ values of $[353 ; 317 ; 323]$.

${ }^{8}$ Figure 8 Panel II presents the mirror Sell*/AA1* relationship example where the coefficients of variation for $\hat{\alpha}_{d}^{\text {Sell*/AA1* }}$ and $\hat{\beta}_{d}^{\text {Sell } * / A A 1 *}$ are $[0.28 ; 0.26 ; 0.30]$ and $[0.30 ; 0.26 ; 0.31]$, which are[1.9; $\left.1.7 ; 2.0\right]$ and $[2.0 ; 1.8 ; 2.0]$ times smaller than those $[0.15 ; 0.15 ; 0.15] B R_{d}^{\text {Sell*/AA1* }}$ values.
} 
$\overline{B R_{d}^{n / m}}$ to be more representative of the daily results than $\overline{\hat{\alpha}_{d}^{n / m}}$ and $\overline{\hat{\beta}_{d}^{n / m}}$ averages, which is fortunate for our next section analysis.

\section{Events dynamics}

In this section, we use our descriptive models estimation results to analyse different aspects of the events arrival dynamics. We also attempt to identify some patterns that may be related to trading strategies and market participant behaviors. In our exponential Hawkes framework, different events relationships patterns are possible. Indeed, it is possible for multiple predecessor events of the same type to combine and increase the arrival rate of a successor event as long as it is possible for this task to be performed by precursor events of different types. We first have the self-exciting case in which, since $n=m$, the $n / m$ effect leads to events of the same type clustering phenomena. We also observe the second situation where $n \neq m$ and the presence of the reciprocal $n / m$ and $m / n$ relationships potentially translate into mutually-exciting situations. We note a third situation in which $n \neq m$ but only one of the $n / m$ or $m / n$ relationships is present. And finally, we observe several situations that we qualify as plurally-exciting in which more than two events are involved in an arrival dependency structure. Taking the simple example of the simple self-exciting case, the same alpha and beta parameters are used to characterize the timing and strength impact of an orphan event occurring in an inter-exciting period as well as for the third event of a cluster. The same applies to another example in which three events are defined: $m \in S$ and $n_{1}, n_{2} \in S^{m *}$ such that $S^{m *} \subseteq S^{m}$. As before, this definition involves the presence of $n_{1} / m$ and $n_{2} / m$ relationships, which we define as plurally-exciting. In this case, we denote essentially five scenarios through which events $n_{1}$ and $n_{2}$ may have an impact on event $m$ arrival. We have the orphan $n_{1}$ or $n_{2}$ event, multiple $n_{1}$ or $n_{2}$ occurrences and finally, the cases where we observe a combination of $n_{1}$ and $n_{2}$ events. Since each of theses events arrival structures may origin from various trading strategies, on a daily basis, the maximum likelihood estimation methodology have to result in the best overall data fitting. Therefore, while some relationships estimated parameters suggest the successor event arrival rate to reach very high levels over small periods, we have to keep in mind that they may be generally part of intensive excitation periods involving several types of predecessor events. Therefore, with some exceptions covered in the next section, we consider generally irrelevant to focus on a predecessor event single occurrence effect on a successor event. Indeed, since dependant on the arrival context, from our point of view, isolating the marginal effect 
of an event $n$ occurrence on event $m$ arrival probability requires too many strong assumptions such as the certainty that no other event affecting the arrival of event $m$ would occur over an arbitrary chosen time period to provide reliable results. In this context, since constant through all possible states, once again, the branching ratio (BR) represent a choice measure for our analysis of the events arrival dynamics.

However, while providing information on a single event $n$ occurrence effect on event $m$ expected number, this ratio provides no information on the total events $n$ involvement in events $m$ arrival over a complete trading day. Therefore, comparing event relationships using BR as the only measure may lead to some misinterpretations. Indeed, for the same $B R_{n / m}$ value, the $n / m$ relationship implications differ given that event $m$ is less, equally, or more frequent than event $n$. In this context, to complement the branching ratio in our relationships comparison, we use the following expression to define the adjusted branching ratio, which is based on Rambaldi et al. (2017) adjusted kernel norm :

$$
\widetilde{B R}_{n / m}=B R_{n / m} \frac{E\left[\lambda_{n}(t)\right]}{E\left[\lambda_{m}(t)\right]}
$$

Under this expression, $B R_{n / m}$ is adjusted through its multiplication by the ratio of event $n$ on event $m$ unconditional expected arrival intensities. The resulting adjusted branching ratio $\widetilde{B R}_{n / m}$ reveals the proportion of event $m$ intensity that may be related to occurrences of event type $n$. In opposition to the previously defined adjusted baseline who provides the proportion of event $m$ arrival that may be related to its constant baseline $\mu_{m}$, the sum of its effects adjusted branching ratios provides the share that may be related to its Hawkes components.

From this point, we mainly focus on the effects that have been considered recurrent enough to be included in our descriptive models. Since they have met our selection criteria at least on every other trading day, we believe these relationships to act as potential pieces of the events arrival dependency structure, which is summarized in Figure 9. Adopting a top-down approach, we begin with Panel I that presents a very general picture of the interrelations among our high level events categories. Afterward, in order to generalize our observations, we work on the basis of relationships classes. As presented in Panel II, these classes group relationships by involved 
successor and predecessor event types and affected book sides. Depending on the involved event categories, such relationship classes may encompass one events effect or more. We assign them an identifier that goes from A1 to S1. Based on the LOB side affected by the involved events, each class is present in two variations. As an example, we consider that Bid LOS effects on Bid LOS and Ask LOS effects on Ask LOS events belong to the dual relationship classes identified P1, which is present twice in Panel II. Once again, because of the strong symmetry observed so far in the relationships affecting the bid and ask LOB sides, we attempt to simplify the presentation by essentially focussing on the buyers' related events. Therefore, while Panel II presents both variations of our relationship classes, a single version is visually represented in Panels III and IV. Panel III exposes the dynamics related to Buy*, Buy and BA1* events. Panel IV performs the same task for Bid LOS w/o BPI and Bid LOC events. Supplementing Figure 9 as the core of the next subsections, for our liquid stocks, Table 5 presents the actual average branching ratios $(B R)$ and adjusted branching ratios $(\widetilde{B R})$ characterizing the individual Hawkes effects relating Trades, LOS w/ BPI and, LOS and LOC events taking place up to depth level 11.

\section{Trades}

As pointed out before, our descriptive models suggest Trades and Trades w/ BPI events arrival to be affected by a limited number of events. Although few in numbers, these effects identified as recurrent by our methodology bring their share of LOB dynamics elements. We relate Buy/Buy* (A1) and Buy/Buy (C1) relationships to the trades clustering phenomena covered by Hewlett (2006). The self-exciting component $\mathrm{C} 1$ suggests the probability of observing a Buy event to be increased after the occurrence of an event of the same type. Similarly, $\mathrm{C} 1$ indicates that following a Buy event occurrence, the probability of Buy* event is also increased. These Hawkes effects being additive, more Buy events taking place within their effective time period lead to even higher Buy and Buy* occurrence probabilities. Despite the fact that they do not take the number of involved shares in each situations into account, $\mathrm{A} 1$ and $\mathrm{C} 1$ are consistent with a trading strategy consisting into splitting the execution of a large parent order into small child orders. We use the simple example of an investor intending to acquire 1000 shares who chooses to perform this task by submitting five consecutive 200 shares child aggressive orders instead of a single 1000 shares order. In a first scenario where the best ask price level contains more than 1000 shares, this execution strategies would result in 5 consecutive Buy events, which is consistent with the self- 
exciting C1 relationship. In a second scenario where the best ask price level contains exactly 1000 shares, four Buy events would be first observed, followed by a Buy* event. In addition to C1, this case would also involve the A1 relation that suggests the short term probability of observing a Buy* event to be increased after the occurrence of a Buy event. Finally, the same effects combination would be involved in a third situation where the best ask price level would contain less than 1000 shares, which would lead to one or more of the child aggressive orders to execute against liquidity available on at least one higher depth level. In this context, the Buy* event would be potentially followed by one or more Buy events that would be part of the whole cluster. This first events arrival dynamics case illustrates the complexity involved in relating our descriptive models results to actual trading situations because of the limited number of parameters available to characterize several complex situations.

As presented in Figure 9, Trades arrival intensity also appear affected by LOS w/ BPI events. Detailed in Panels II and III, AA1*/Buy* (B2) and AA1*/Buy (D1) suggest an increase in the expected number of Trade with and without BPI after the submission of an ask limit order inside the spread creating a new best price ask depth level. On the other hand, BA1*/Buy* (B1) suggests a similar phenomenon to follow the arrival of a bid limit order inside the spread. Unlike previous A1 and C1 relationships, our liquid stocks descriptive models present some discrepancies when it comes to the effects of LOS w/ BPI. While B2 have appeared persistent enough to be included in our models for each of our liquid stocks, B1 have not met our selection criteria for SAP, with the consequence of not making its descriptive model. The same applies to D1 that is not part of the ADS model. Nevertheless, we examine the interpretation of these last two effects insofar as, in addition to having satisfied our selection criteria for two of the three stocks, back to Table 2, we do not observe an absolute absence of relationship regarding the third one. With the most important $\mathrm{BR}$ values among these relationships categories, we relate the persistent B2 relationship to situations where the submission of a best price improving ask limit order (AA1*) would be followed by its rapid total execution (Buy*). In the same way, we relate D1 to the eventual partial consumption of a similar incoming limit order. When considered with $\mathrm{C} 1$ and $\mathrm{A} 1$, it is possible to relate D1 and even B2, to a Buy events cluster that may eventually lead to the total consumption of the new price level. Finally, we relate B1 to a different dynamics. In this case, we consider the mid-quote price increase related to a new best bid depth level as an incentive to acquire the liquidity available at the best ask price quickly. From Table 5 Panel I, it is interesting to note that 
despite smaller $B R$ values, the $\widetilde{B R}$ values ranges for $\mathrm{B} 1, \mathrm{~B} 2$ and $\mathrm{D} 1$ are comparable to those of $\mathrm{A} 1$ and C1, which suggests the contribution of these Hawkes effects in the Trades arrival processes to be similar. From equation (23), it is possible to relate this observation to the fact that BA1* and AA1* are more frequent than Buy* and Buy, which appear to compensate for their more limited individual occurrence effects.

\section{Limit orders submissions with best price impact}

Panels II and III of Figure 9 shows that LOS w/ BPI arrival rate may be affected by the occurrence of events from our four main categories. From a mechanical point of view, it is not surprising to note that, as indicated by their BR values in Panel II of Table 5, Trades w/ BPI appear to have the more important events occurrence individual effects. These events resulting in an increased bidask spread, more room becomes available for the creation of a new best price level on both side of the book. Despite the fact that our models do not explicitly take the magnitude of the spread growth into account, we know for sure that it corresponds to at least one tick. First, we consider the highly persistent (see Table 2) Buy*/BA1* (E1) relationship as a part of a price following dynamics in which the mid-quote price increase caused by the Buy* event may be followed by the creation of a new best bid price level. On the other hand, we consider Sell*/BA1* (E2) as part of a LOB resilience dynamics where the total execution of the previous best bid price level resulting in a Sell* event may be followed by the creation of a new one, through a limit order submission that would be identified as a BA1* event.

When it comes to Trades w/o BPI effect on BA1* (F1), we consider this relationship in a mutuallyexciting context. Indeed, because of the Hawkes effects additive nature and the previously described clustering phenomena related to Buy event that may lead to a Buy*, we consider possible for Buy events to be part of potential dynamics leading to the formation of a BA1* event favorable environment, once again, in a price following context. Despite less important in terms of individual events occurrence effect, G1 to I1 indicates that some LOS and LOC events taking place around the best price levels may have effects on BA1* arrival. First, BA1*/BA1* (G1) suggests the possibility for BA1* events to occur in clusters. We consider successive BA1* events consistent with a book liquidity replenishment situation that may follow, as an example, a Sell* event (E2) resulting from a transaction having consumed more than one bid price levels. Regarding BA1/BA1* (H1), we consider the presence of its reciprocal BA1*/BA1 (N1) relationship (see 
Table 5 Panel II) as an indicator that BA1 and BA1* events occurrence may be favorited under similar circumstances. Both event types involve added liquidity on the best bid price level, actual or new. Finally, we relate I1 and I2 to the bid-ask spread enlargement that may result from BC1 and $\mathrm{AC} 1$ events in situations where the cancelled limit order is the only constituent of the best price level. Shown in Table 5 Panel II, we consider the total absence of BA1*/BC1 and AA1*/AC1 relationship for our liquid stocks as an indication that, in general, the cancellation of limit order creating a new best bid or ask price level does not immediately follow its submission.

\section{LOB events}

Having highlighted some interesting characteristics of Trades and LOS w/ BPI events arrival process, the main goal of this section is to perform a similar analysis for LOB events. Depending on the predecessor events, we observe relationships involving LOS w/o BPI and LOC as the successor event to present different characteristics. First, we study the effects of Trades and LOS w/ BPI on LO submissions and cancellations. Afterward, we achieve a similar task through the analyze of LOB events effects on each other arrivals. Finally, we take a look at each of these event categories contribution to the LOB events arrival.

Figure 10 presents the effects of Trades w/ BPI, Trades w/o BPI, and LOS w/ BPI on LOS w/o BPI and LOC events taking place on depth levels 1 to 20 in terms of branching ratios. In line with our previous visual representations, J1 to K2 relationship classes are detailed through the inner graphics displaying the corresponding labels. Still working from the buyer point of view, we focus on Panels I, III and V that exhibit the effects of a single occurrence of Buy*, Buy and BA1* events. By concentrating on these predecessor events similarities, differences, and effects on LOB events arrival, we attempt to characterize the dynamics surrounding their occurrence. Basically, we relate the Buy*, Buy and BA1* events through the fact that they all result from an aggressive buyer action. Consuming at least one complete price level, we consider a Buy* event as very aggressive. Similarly, resulting from the partial execution of the best price level, we qualify the Buy event as aggressive. And, although it does not immediately lead to an actual transaction, since it results from the submission of the most aggressive limit order at a given time, we consider BA1* to immediately follow the two former events on our aggressiveness scale. When not related to only one of these events, the other properties relevant to our dynamics description are shared on an events pair basis. First, while Buy* and Buy affect the ask side of the book, BA1* have an impact 
on the bid side. Second, Buy* increases, BA1* decreases, and Buy has no effect on the bid-ask spread. Third, unlike Buy that does not affect the price structure, by respectively leading to a change in the best ask and bid prices, Buy* and BA1* increase the mid-quote price. When it comes to the effects on LOB events arrival, we identify these characteristics as more important than the actual liquidity execution involved in Buy* and Buy. Figure 10 suggests BA1* effects on LOB events arrival to be more important in numbers and in intensity than those related to Buy. However, one may expect Buy event effects to be closer to these of Buy* since they both involve actual liquidity execution, which appears inaccurate according to our descriptive models results. Indeed, while Buy* stands out as the most important predecessor event in terms of individual occurrence effects, it is immediately followed by BA1*. Since Buy is left behind, we identify the mid-quote price increase and the change in the LOB structure specific to the other two events as important determinants of the events arrival dynamics. With these LOB impacts in mind, we first distinguish two sets of similar relationship classes involving our three predecessor events. While J1, L1, and $\mathrm{N} 1$ suggest Buy*, Buy, and BA1* to affect bid LOS arrival, K2, M1, and $\mathrm{O} 2$ do the same regarding ask LOC.

Beginning with bid LOS, as shown in Figure 10, the most important effects in terms of number of affected events and branching ratios belong to J1 and N1. These relationships suggest Buy* and BA1* events to be immediately followed by increased probabilities of bid LO submissions respectively taking place on depth levels 1 to 5 and 1 to 7 . We relate these effects to eventual buyers submitting their limit orders in an environment where the stock price may appear to be increasing. Indeed, Buy* and BA1* events being accompanied by a mid-quote price increase, we consider these potential buyers to submit their limit orders behind this new price, in an attempt to follow this possible upward trend. At this point and all over the current analysis, we have to keep in mind that we denote increases in the expected number of bid LOS events following Buy* and BA1* occurrences, which highly differs from an actual systematic limit orders flow simultaneously taking place on these multiple depth levels. We assume that the depth levels on which such potential limit orders would be submitted would be determined by their owners patience level and trading strategy. Regarding the Buy predecessor event, as presented in Figure 10 Panel III, L1 suggests it only affects bid LOS arrival taking place on depth levels 1 to 3 through effects that present smaller BR values than those attached to Buy* and BA1*. We relate these less important effects to the previously described absence of individual Buy event structural LOB 
effect. The transactions leading to the identification of these events only partially affect the best ask price level. However, we relate their presence to the fact that, as for Buy*, Buy events occurrence may suggest the presence of an impatient buyer, which may sometime be interpreted by patient sellers as a potentially increasing price signal. Also, we have to consider the previously established self-exciting nature of the Buy event that could lead to an addition of these effects over short time periods.

Regarding the second group of relationships similar for our three predecessor events, as claimed before, $\mathrm{K} 2, \mathrm{M} 1$ and $\mathrm{O} 2$ suggest Buy*, Buy and BA1* to have an effect on ask LOC events arrival on various depth levels. These events potentially carrying signs of an increasing price, we consider the fact that they appear to favor the cancellations of sell limit orders intriguing. Indeed, it suggests that some market participants tend to cancel their limit orders as their execution probability increases. From our point of view, this phenomenon may be related to different situations. First, we consider possible for some orders to be owned by market makers with the intention of providing liquidity in the LOB without a real interest in seeing their orders executed. Second, they may be part of complex trading strategies potentially involving LO submissions and cancellations on both sides of the book. Finally, Buy*, Buy and BA1* suggesting the presence of impatient buyers, we consider possible for patient sellers already present on the ask side to cancel their limit order in an attempt to take more advantage of the potentially increasing price. In this last case, the cancelled limit orders would be eventually resubmitted with higher prices. Although Figure 10 suggests these relationships to be related to Buy*, Buy and BA1* events, it indicates Buy* to have the most important underlying effects. Indeed, while appearing to affect ask LOC events up to the ninth depth level, Buy* effects seem atypically strong for LOC taking place on levels 1 to 3. Actually, with average branching ratios generally above 0.8 , the highest BR values observed in our entire system belong to Buy* effects on $\mathrm{AC} 1$ and $\mathrm{AC} 2$ events arrival. We relate this important $\mathrm{K} 2$ relationship class to the fact that the Buy* event directly affect the ask side of the book by completely consuming one or more price levels, which is not the case for Buy and BA1*. A Buy* event occurrence has for consequence that all existing ask limit orders rank relative to the best ask price is improved by the number of price levels actually consumed by the underlying transaction. In our framework context, this rank correspond to the depth level number. No matter the trading strategies behind $\mathrm{K} 2$ effects, it is a certainty that the potentially involved limit orders have become 
closer to the best ask price before their cancellation. In this context, we consider possible for some systematic trading strategies to use limit orders depth level as a trigger to launch their cancellations.

As presented through $\mathrm{J} 2$, Buy* events favor ask LOS through numerous relationships which, in some cases, may also be qualified as important in BR values terms. While observed up to depth level 16, these effects present more important BR values for AA1 to AA10. Back to Figure 10 Panels III and IV, we observe a complete absence of similar effects involving Buy and BA1*. In line with a possible upward price trend, we relate these increased probabilities to eventual patient sellers submitting limit orders in an attempt to obtain an even better price than the actual new best ask price. The different depth levels over which the LOS probabilities are affected suggest various levels of patience, which may depend on the concerned investors' characteristics and their trading strategies. Considering the previous scenario where a still patient seller would attempt to take a chance at a possibly increasing price. In an ask side reorganization context, a cancelled ask limit order could be submitted on a higher depth level. Despite speculative because of the lack of information sequentially relating $\mathrm{K} 2$ and $\mathrm{J} 2$, a part of $\mathrm{J} 2$ induced probability increases could be attributed to these resubmitted orders.

We end the analysis of Trades and LOS w/ BPI impacts on LOB events arrival with relationship classes K1 and O1, which correspond to Buy* and BA1* effects on bid LOC. Panel III shows such effects to be totally absent when it comes to the Buy predecessor event. While $\mathrm{K} 1$ and $\mathrm{O} 1$ involve bid LOC probabilities increases up to depth level 19, we relate the absence of such effect regarding Buy to the fact that by definition, it does not change the best bid and ask prices and by extension, the mid-quote price. We relate bid LOC events that may arise in the $\mathrm{K} 1$ and $\mathrm{O} 1$ contexts to situations where limit orders would be cancelled by buyers observing an adverse move in the stock price. The unusually high number of depth levels over which LOC probabilities appear affected suggests various patience levels among the potentially concerned investors over time. Regarding $\mathrm{K} 1$, similar to what happens on the ask side where existing orders become closer to the best ask price as represented by $\mathrm{K} 2$, a Buy* event has for consequence that the limit orders already present on the bid side become farther from the best ask price. Therefore, depending on an investor patience level and trading strategy, a given distance from the best ask or mid-quote price may act as a threshold to trigger a limit order cancellation. From our point of view, unlike K2 where the cancelled limit orders were getting too close to the action for still patient sellers, K1 cancelled limit 
orders seem to get too far from the action to be kept alive by patient buyers seeing their execution probability reduced. In the $\mathrm{O} 1$ case, we observe some of the effects to be characterized by BR larger than those seen in K1. We explain this situation by the fact that unlike Buy* which affects the ask side of the book, BA1* events have a direct impact on the bid side through the addition of a new depth level that becomes the best price level. All existing bid limit orders are then affected by being automatically shifted one price level away from the best bid price. These effects strength suggests a limit order distance from its book side best price to be more monitored and used as a cancellation trigger than its distance from the opposite book side best price. Moreover, we consider the $\mathrm{BA} 1 * / \mathrm{BC} 11$ important $\mathrm{BR}$ value as an indication that the $11^{\text {th }}$ price level relative to the best price may be used by several algorithms as a threshold to determine that a limit order has become too far away (above level 10) from the action and, must be cancelled. We relate this strong relationship to the particularly good data fitting performance of our descriptive models regarding BC11 and AC11 (see Figure 4), which suggests an adequate representation of this dynamics. Additionally, $\mathrm{O} 1$ graphic suggests that reaching depth levels 2, 4 and 6 could also be single out as limit orders cancellation triggers.

Regarding LOB events effects on each other arrival, we summarize these impacts through relationship classes P1 to S1. Figure 10 Panel IV provides a general idea of how bid LOS and LOC are related to other events of the same classes taking place on both sides of the book. In the previously described cases of Trades and LOS w/ BPI events, the absence of reciprocal counterparts to relationship classes $\mathrm{J} 1$ to $\mathrm{O} 2$ allowed the assumption of some forms of causality. While Trades and LOS w/ BPI appear to affect the arrival of LOB events in different ways, only few relationships going the other way have been observed. Indeed, with the exceptions of H1 to I 2 that suggest best price levels LOS w/o BPI and LOC effects on BA1* and AA1* arrival, Trades and LOS w/ BPI generally appear unaffected by LOB events. However, it is possible to observe that some of the P1 to S1 relationship classes, which involve LOB events only, act as each other reciprocal. The resulting mutual-excitation phenomenon affect various events sets going from pairs of individual events of the same type to events groups of different types taking place on both sides of the book. By grouping extracts from Table 5, Table 6 summarizes the two most extended cases of mutually exciting zones present in our events dependency maps. While Panel I presents interrelations among bid LOS and ask LOC, Panel II does the same regarding ask LOS and bid LOC. Insofar as this phenomenon appears concentrated on the first three price levels, we solely 
focus on this segment of the LOB. Panel I shows that for each of our three liquid stocks, multiple relationships are present in the $\mathrm{BA} 1$ to $\mathrm{BA} 3$ / $\mathrm{AC} 1$ to $\mathrm{AC} 3$ zone, which correspond to the ask $\mathrm{Q} 2$ relationship class. In a reciprocal way, the bid $\mathrm{R} 2$ class reveals several effects in the $\mathrm{AC} 1$ to $\mathrm{AC} 3$ / BA1 to BA3 zone. The situation increases in complexity when we also consider the bid P1 and ask $\mathrm{S} 1$ relationship classes that correspond to $\mathrm{BA} 1$ to $\mathrm{BA} 3$ / $\mathrm{BA} 1$ to $\mathrm{BA} 3$ and $\mathrm{AC} 1$ to $\mathrm{AC} 3$ / $\mathrm{AC} 1$ to AC3. Although the involved individual effects do not present particularly high BR values, we conclude in an important level of interrelation among them. While Table 6 examples generally exhibit some individual relationships absence, Panel II shows total interconnection among AA1 to $\mathrm{AA} 3$ and $\mathrm{BC} 1$ to $\mathrm{BC} 3$ events in the $\mathrm{BMW}$ case. In this specific situation, all events occurrences seem to affect their own event type arrival and the arrival of each of the other event types present in the set. Although not all involving total interconnection, each Table 6 example involves a complex multi-event mutually excitation chains whose detailed interpretation may become very challenging. Consequently, in such situations, it becomes difficult to go further than assuming that the involved events tend to occur under the same circumstances. It is important to note that these circumstances may involve the occurrence of Trades and LOS w/ BPI events, or not. Indeed, although possible that $\mathrm{J} 1$ to $\mathrm{O} 2$ capture an important part of the Trades and LOS w/ BPI effects on LOB events, in our exponential Hawkes models context, P1 to S1 rely on the same parameters set to describe dependency among LOB events arrival whether or not they are part of a dynamic related to $\mathrm{J} 1$ to $\mathrm{O} 2$. However, Trades and LOS w/ BPI events being generally less frequent than LOB events, we consider possible for P1 to S1 relationship classes to be more related to situations taking place outside these events dynamics.

As detailed through Panels III to VI, relationship classes P1 to S2 sometime have a black box appearance. However, although impossible for us to interpret each individual effect, we are still able to observe some general patterns. First, reminding the complete absence of Buy/Sell*, Sell/Buy*, Buy/Sell and Sell/Buy relationships, we denote the almost total nonappearance of recurrent relationships between bid and ask LOS events. A similar situation is observed regarding bid and ask LOC events, which occurrences also appear unrelated. It is true that we have observed the pairs of relationships $(\mathrm{J} 1, \mathrm{~J} 2),(\mathrm{K} 1, \mathrm{~K} 2)$ and $(\mathrm{O} 1, \mathrm{O} 2)$ that suggest some of these events to tend to follow the same Trades and LOS w/ BPI events. However, we consider that the difference in the BR values patterns specific to these relationships pairs consistent with this absence of interrelation among the affected events. As claimed before, these absences suggest opposite sides 
LOS and LOC to occur generally under distinct circumstances. Since these two class of events suggest different strategies and reading of the market conditions, it would be consistent for market participants to do not tend to perform the underlying actions concurrently. At the opposite, P1 suggests that LOS taking place on the same side of the book tend to occur in similar contexts. As exposed by S1, the same phenomenon appears to apply to same side LOC events. As shown in Panels III and V, for both P1 and S1, the intensive mutual-excitation zone previously described appears to take place up to depth level four. Beyond this point, the same side LOS/LOS and LOC/LOC recurrent effects appear concentrated around the diagonal, which suggest that individual effects mostly relate events taking place on nearby depth levels. While same side LOC/LOC relationships appear diffuse and tend to vanish around depth levels 6 to 8 , we observe recurrent LOS/LOS effects to follow a straight line up to depth level 10. It is interesting to note that this line is different from the actual diagonal that would encompass self-exciting Hawkes effects through which LOS events taking place on a given level would tend to follow each other. In fact, we observe an offset in the BA3 to BA9 / BA4 to BA10 line that suggests LOS occurrences on a given depth level to be followed by an increase in the arrival probability of LOS taking place one level higher. The depth level number being relative, it is unfortunately impossible for us to establish whether there is a price difference between the orders involved in these relationships or not.

Regarding relationships involving limit orders submission and cancellation events taking place on the same book side, since they respectively encompass LOS/LOC effects and their reciprocal counterparts LOC/LOS, for a given book side, we consider Q1 and R1 relationship classes as paired. Back to Table 5, Panels III to VI show that R1 includes more recurrent effects than Q1, which suggests a tendency for LOS events to follow LOC. This is consistent with our previous hypothesis suggesting that certain systematic strategies may reposition limit orders following certain changes in market conditions that may be driven by Trades w/ BPI and LOS w/ BPI. Finally, as introduced before, Q2 and R2 relate LOS and LOC events taking place on both sides of the LOB. As presented in Panels III to VI, each of these relationship classes involves an important mutual excitation zone that generally extends up to the third or fourth depth levels. Back to Figure 10, we have seen that relationship class pairs (J1, K2), (L1, M1) and (N1, O2) suggest a tendency for opposite sides LOS and LOC events to follow the same Trades and LOS w/ BPI events. The pairwise BR values patterns presenting similarities, we consider these Trades and LOS 
w/ BPI events arrival as circumstances under which these LOS and LOC events may tend to occur together. Taking place inside these contexts or not, these aggregated reciprocal relationships remain difficult to interpret since as usual, it is impossible to establish if the LOS and LOC events origin from the same market participants or not. However, they would be consistent with any strategy involving the cancellation of limit orders present on one side of the book and submission of new ones on the other side. As for all dynamics discussed in this section, the exact motivation and timing of such actions would depend on their trading strategies.

Having focused on specific types of events relationships, we close this section with an overview of global event categories contribution to the arrival of LOB events. Figure 11 shows the average proportions of Trades w/ BPI, Trades w/o BPI, LOS w/ BPI and LOB events to the arrival of LOS w/o BPI and LOC events. For a given category, this measure sums the adjusted branching ratio of the included effects and reports the daily average. No distinction is made between the effects involving predecessor events impacting the LOB bid side and those affecting the ask side. These results are related to those presented in Figure 5 as for each successor event and trading day, the effects contribution sum essentially equals to one minus the adjusted baseline value. Proceeding in the usual order, we first observe a general steadiness regarding Trades w/ BPI contribution to the arrival of both LOS w/o BPI and LOC events taking place on depth levels 1 to 8. Beyond this point, we note a general increase in this contribution that afterward, remains generally constant until it vanishes among the highest considered levels. It is interesting to note that despite some very important individual effects, the contribution of Trades w/o BPI appears relatively small. Buy* effects on AC1 and AC2 represent good examples of this situation. Back to Figure 10 Panel I, we have seen that they present the most important individual events occurrence impact with average branching ratio values of [0.81 and $0.85 ; 0.83$ and $0.92 ; 0.74$ and 0.79$]$. However, when it comes to contribution, the average adjusted branching ratios fall to [0.11 and $0.10 ; 0.11$ and 0.10 ; 0.11 and 0.12]. Using expression (23), it is possible to relate this phenomenon the relative infrequence of the Buy* event with regard to BC1 and AC1. This applies to Trade w/ BPI effects on LOS w/o BPI and LOC events taking place on several depth levels. In fact, because of their generally significant individual effects on LOB events and small relative number of occurrences, we identify Trade w/ BPI effects as periodical LOB activity boosters. On the other hand, in line with their small individual impacts, we denote a marginal Trades w/o BPI contribution to LOB events arrival. 
When it comes to the LOS w/ BPI contributions to LOS w/o BPI and LOC events arrival, although appearing more important for cancellations, they remain generally similar for both categories regarding events occurring on depth levels 1 to 5. Beyond this point, while rapidly vanishing for LOS w/o BPI, for LOC they show increasing trends as the distance from the best prices also increases. From our point of view, these phenomena suggest that even on the deepest depth levels, orders cancellations may be more related to automatic actions than orders submissions. The important LOS w/ BPI events contribution to these LOC events may once again be related to systematic trading strategies monitoring their own limit orders rank relative to the same or opposite book side best price. And, based on their rank, take the decision to cancel limit orders or not. A good representation of this mechanics could be related to the fact that as seen before, our descriptive models seem to deliver a better fitting performance for LOC than LOS w/o BPI events, especially on the deep price levels.

Back to Figure 11, we note a similarly important aggregated LOB events contribution to the arrival of both LOS w/o BPI and LOC events taking place on depth levels 1 to 5. Despite the previously presented small individual LOB events / LOS w/o BPI and LOB events / LOC relationships contribution, since these effects are numerous to be part of our descriptive models, once combined, they represent the main drivers for this segment of the dynamics. Beyond depth level 5, their cumulated contribution to LOS w/o BPI and LOC arrival becomes different. Indeed, while remaining important determinants of LOS w/o BPI arrival at least up to depth level 10, their contribution to LOC arrival decreases until it vanishes around depth level 8.

\section{Conclusion}

In this paper, we analyze the limit order book events arrival dependency structure. Our set of potentially interconnected events consists in Trades, Limit Order submissions and Limit Order cancellations taking place on the first twenty depth levels of the book.

At every step of our process, we observe a generalized symmetry regarding events affecting the bid and the ask sides in terms of Hawkes effects recurrence and strength. We consider this as a sign that similar trading strategies probably operate on both sides of the book. Similarly, despite some idiosyncrasies among our three stocks, we note that the absent and the highly recurrent relationships generally turn out to be the same for each of them. 
Once our descriptive models estimated, we find that beyond depth levels 1 to 6 where our descriptive models offer a satisfactory data fitting performance for order submissions and cancellations, this performance shows a general decreasing trend as we move in events depth level of events occurrence. On the same depth levels, the Poisson homogenous comparative models show an improving fitting performance. We relate this performance convergence to a decrease in the Hawkes effects contribution to the concerned events arrival that comes together with an increase in the constant baseline contribution.

Through the investigation of the global events arrival dynamics, we confirm that while trades and limit order submissions having an effect on the best price sometimes affect limit order submissions and cancellations up to the deepest segment of the order book, these events appear almost totally unaffected by events taking place beyond the second depth level. We show that while both trades and limit order submissions with best price impact may affect limit order book submissions and cancellation up to the highest depth levels, the effect of their counterpart events without best price impact is less extended.

We also find that both in terms of individual occurrence effects and global contribution, limit order cancellations appear particularly affected by the events having a best price impact. We relate this observation to the possibility for cancellations to be systematically launched on the basis of criteria that may be well represented by our models. With their branching ratios that may be considered as outliers, we consider the case of the effects of limit order submissions with best price impact on limit order cancellations taking place on the depth level 11 of the same book side as particularly revealing on the matter. Indeed, we explain these very strong relationships by the possibility for some trading strategies to mark the $10^{\text {th }}$ depth level as a the farthest from the best price a limit order can be before its cancellation is automatically triggered.

Finally, we observe segments of our events dependency structure over which several event types appear to affect each other's arrival, which we qualify as mutually-exciting zones. Based on the involved events type and the relationships characteristics, in these cases, we rule out the causality and consider these events as potentially related to the same factors.

In the forthcoming extension, we will use the elements presented in this paper as the foundations for a complete limit order books simulator in which the different types of event arrival will be 
dictated by Hawkes processes models. The current paper having mainly focussed on the events arrival, we now have to concentrate on the characteristics of the orders underlying to these events such as the number of shares included in a submitted limit order or consumed by a trade. We also have to define some dynamics such as the choice of the order targeted by a limit order cancellation assuming several orders standing on the concerned depth level. Once these issues figured out, we expect to use real limit order book states as starting points for the simulations. Then, it will be possible to employ different sets of daily estimated parameters to produce and analyze prices trajectories. 


\section{References}

Almgren, Robert F. (2003). "Optimal execution with nonlinear impact functions and tradingenhanced risk." Applied Mathematical Finance 10(1): 1-18.

Almgren, Robert F., Neil, Chriss (2000). "Optimal execution of portfolio transactions." Journal of Risk 3(2): 5-39.

Bacry, E., S. Delattre, M. Hoffmann and J. F. Muzy (2013). "Modelling microstructure noise with mutually exciting point processes." Quantitative Finance 13(1): 65-77.

Bertsimas, Dimitris and Andrew W. Lo (1998). "Optimal control of execution costs." Journal of Financial Markets 1(1): 1-50.

Bowsher, Clive G. (2007). "Modelling security market events in continuous time: Intensity based, multivariate point process models." Journal of Econometrics 141(2): 876-912.

Carstensen, Lisbeth (2010). PhD thesis Hawkes processes and combinatorial transcriptional, University of Copenhagen.

Cheng, Xue, Marina Di Giacinto and Tai-Ho Wang (2017). "Optimal execution with uncertain order fills in Almgren-Chriss framework." Quantitative Finance 17(1): 55-69.

Cont, Rama, Sasha Stoikov and Rishi Talreja (2010). "A Stochastic Model for Order Book Dynamics." Operations Research 58(3): 549-563.

Embrechts, Paul, Thomas Liniger and Lu Lin (2011). "Multivariate Hawkes processes: an application to financial data." Journal of Applied Probability 48(A): 367-378.

Fauth, Alexis; Tudor, Ciprian A. (2012) "Modeling First Line Of An Order Book With Multivariate Marked Point Processes." eprint arXiv:1211.4157.

Hawkes, Alan G. (1971). "Spectra of Some Self-Exciting and Mutually Exciting Point Processes." Biometrika 58(1): 83-90.

Hawkes, Alan G. and David Oakes (1974). "A Cluster Process Representation of a Self-Exciting Process." Journal of Applied Probability 11(3): 493-503.

Hewlett, Patrick (2006). "Clustering of order arrivals, price impact and trade path optimisation." $\underline{\text { In Workshop on Financial Modeling with Jump processes, Ecole Polytechnique. }}$

Huang, He (2012). Modeling Order Book Dynamics Using Queues And Point Processes. Ph.D., The Florida State University.

Huang, Weibing, Charles-Albert Lehalle and Mathieu Rosenbaum (2015). "Simulating and Analyzing Order Book Data: The Queue-Reactive Model." Journal of the American Statistical Association 110(509): 107-122. 
Large, Jeremy (2007). "Measuring the resiliency of an electronic limit order book." Journal of Financial Markets 10(1): 1-25.

Lu, Xiaofei and Frédéric Abergel (2018). "High-dimensional hawkes processes for limit order books: Modelling, empirical analysis and numerical calibration", Quantitative Finance, 18(2): 249264.

Moallemi, Ciamac C. and Mehmet Sağlam (2013). "Or forum - the cost of latency in highfrequency trading", Operations Research, 61(5): 1070-1086.

Muni Toke, Ioane and Nakahiro Yoshida (2017). "Modelling intensities of order flows in a limit order book." Quantitative Finance 17(5): 683-619.

Obizhaeva, Anna A. and Jiang Wang (2012). "Optimal trading strategy and supply/demand dynamics." Journal of Financial Markets 16(1): 1.

Ogata, Y. (1981). "On Lewis' simulation method for point processes." IEEE Transactions on Information Theory 27(1): 23-31.

Rambaldi, Marcello, Emmanuel Bacry et Fabrizio Lillo (2017). "The role of volume in order book dynamics: A multivariate hawkes process analysis", Quantitative Finance, 17(7): 999-922.

Shek, H.H. (2011). "Modeling high frequency market order dynamics using self-excited point process." SSRN Electronic Journal.

Toke, Ioane Muni (2011). "Market Making" in an Order Book Model and Its Impact on the Spread. Econophysics of Order-driven Markets: Proceedings of Econophys-Kolkata V. F. Abergel, B. K. Chakrabarti, A. Chakraborti and M. Mitra. Milano, Springer Milan: 49-64.

Toke, Ioane Muni and Fabrizio Pomponio (2012). "Modelling trades-through in a limit order book using hawkes processes." Economics - The Open-Access, Open-Assessment E-Journal 6(22): 123.

Vinkovskaya, Ekaterina (2014). A point process model for the dynamics of limit order books. Dissertation/Thesis.

Zheng, Ban, François Roueff et Frédéric Abergel (2014). "Modelling bid and ask prices using constrained hawkes processes: Ergodicity and scaling limit", SIAM Journal on Financial Mathematics, (5): 99-136. 
Figure 1 LOB inter-updates durations empirical CDF

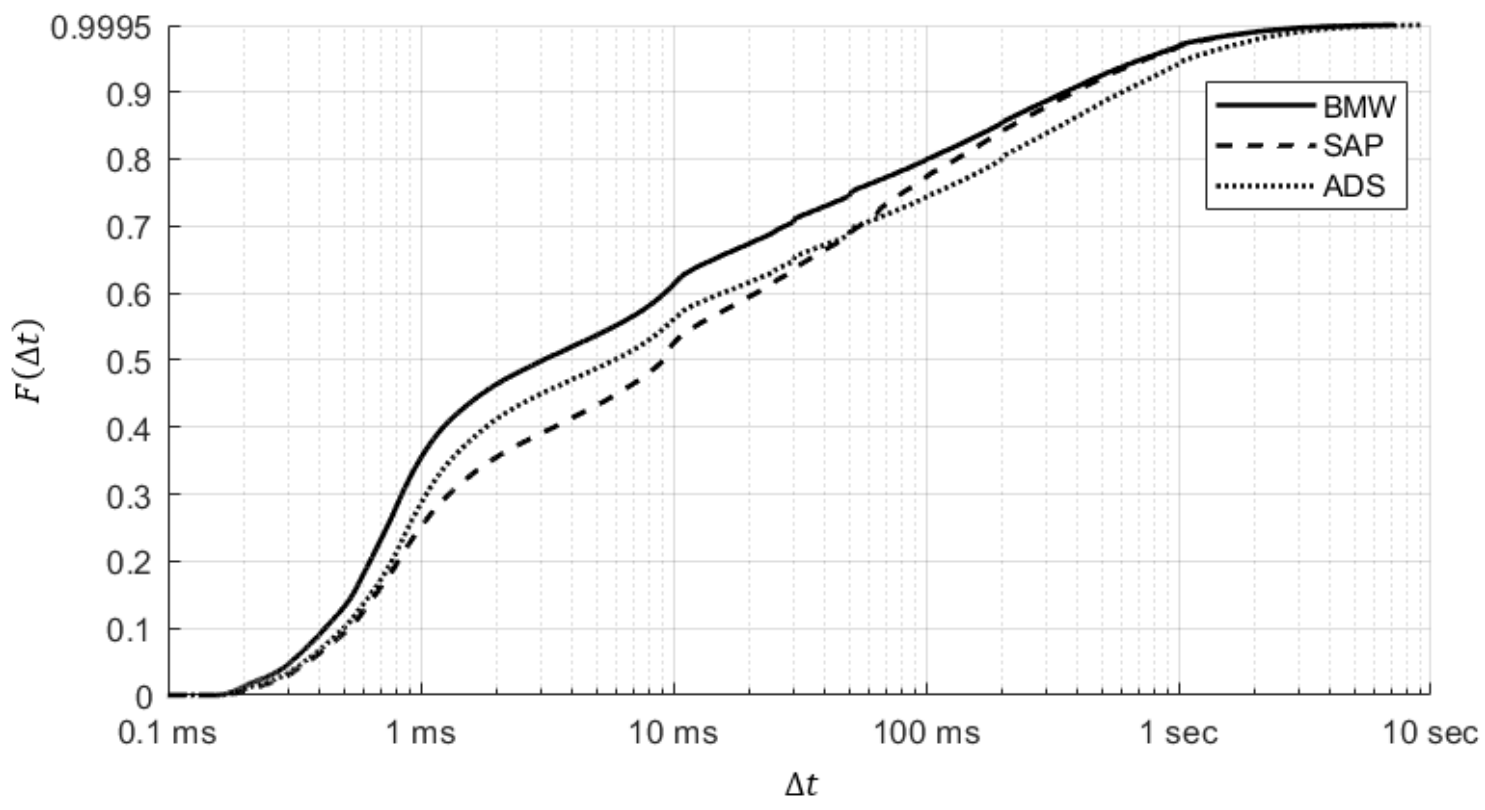




\section{Figure 2 Fictional events examples and their LOB effects}

Panel I : Buy trade with best price impact (Trade w/ BPI) - Buy*

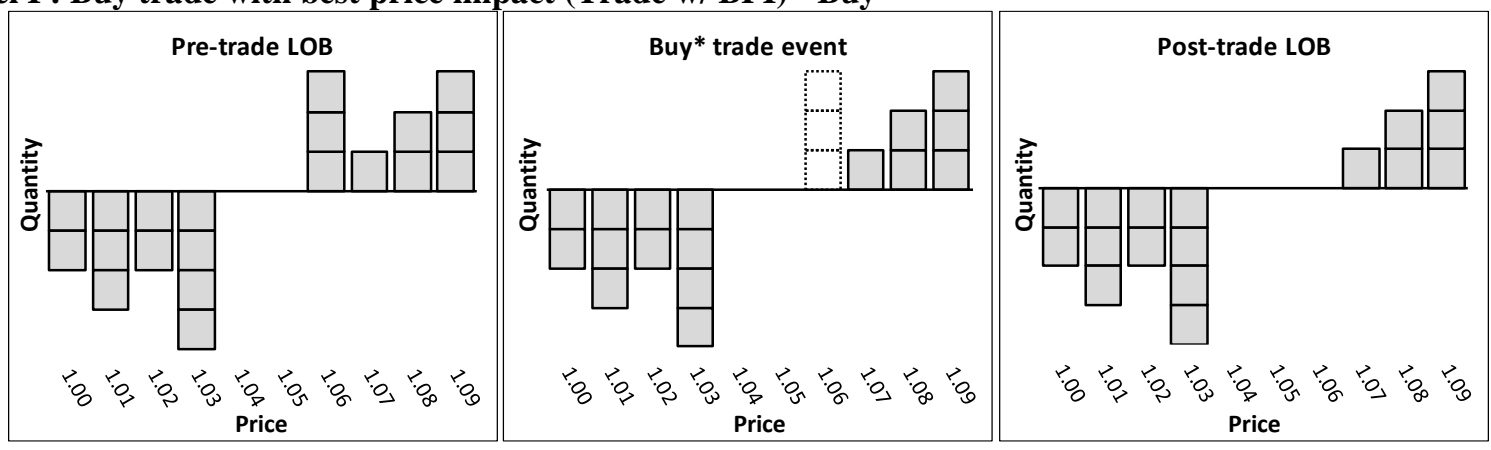

Panel II : Buy trade without best price impact (Trade w/o BPI) - Buy

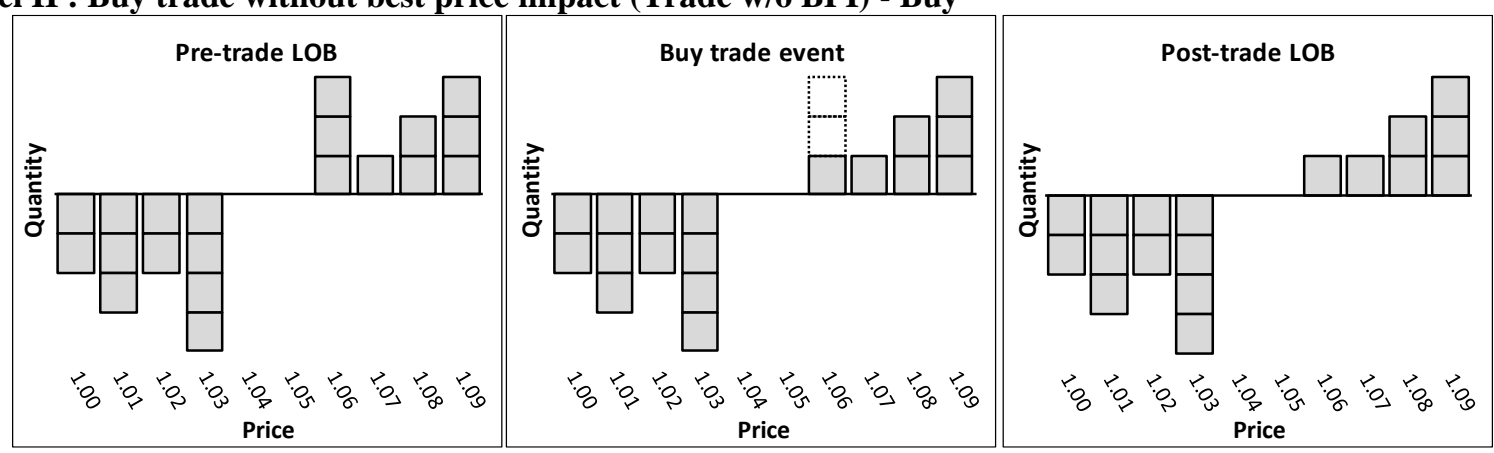

Panel III : Ask limit order submission with best price impact (LOS w/ BPI) - AA1*

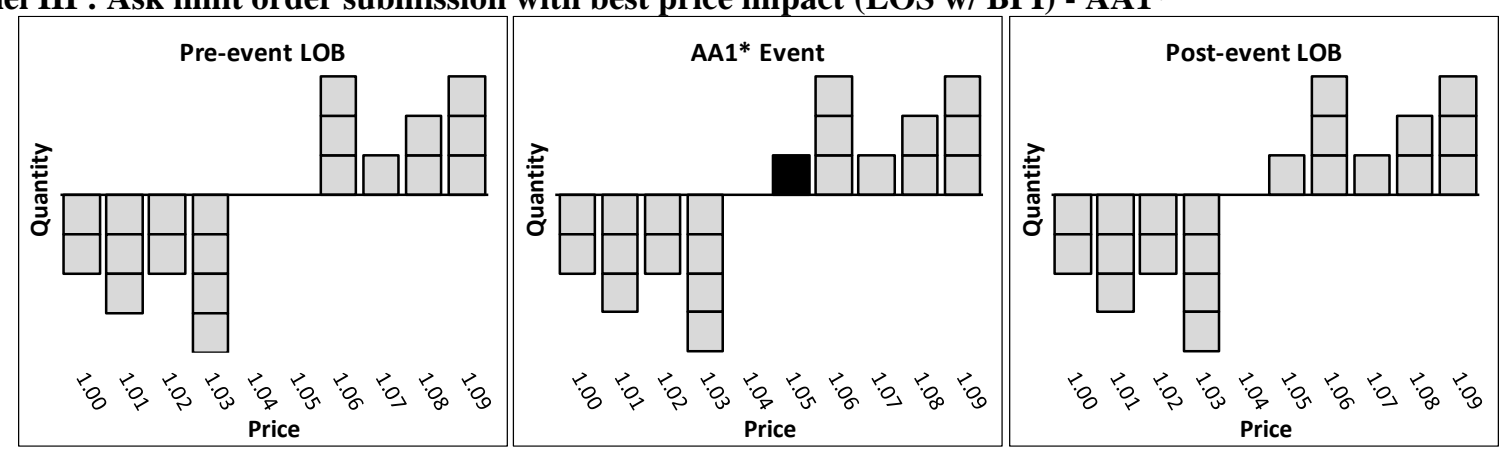

Panel IV : Ask limit order submission on depth level 2 (LOS w/o BPI) - AA2 - Case 1

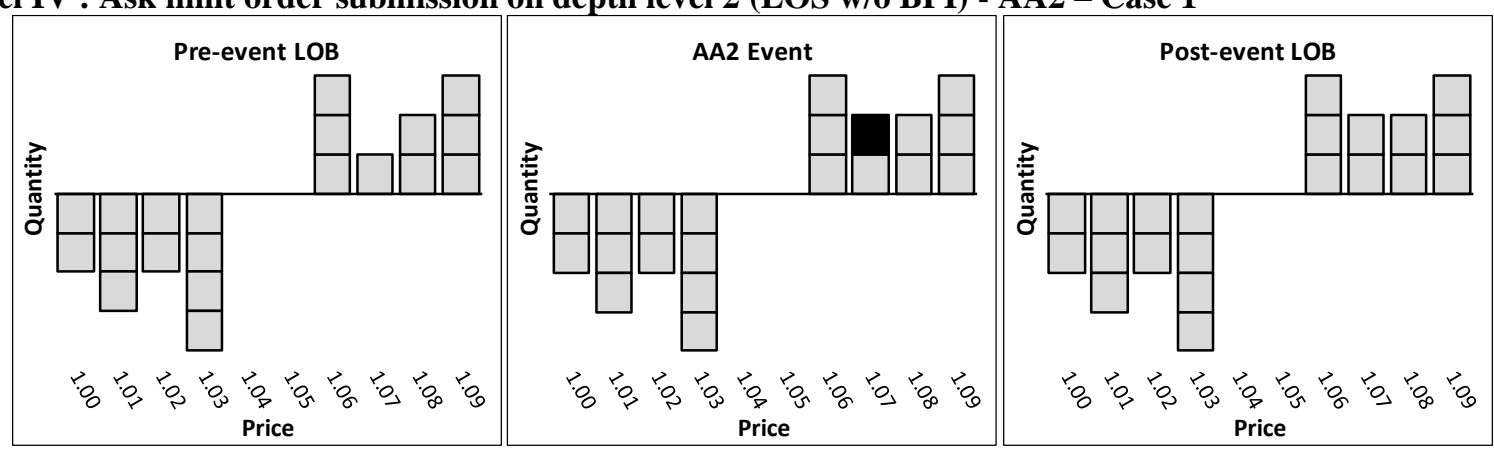


Panel V : Ask limit order submission on depth level 2 (LOS w/o BPI) - AA2 - Case 2

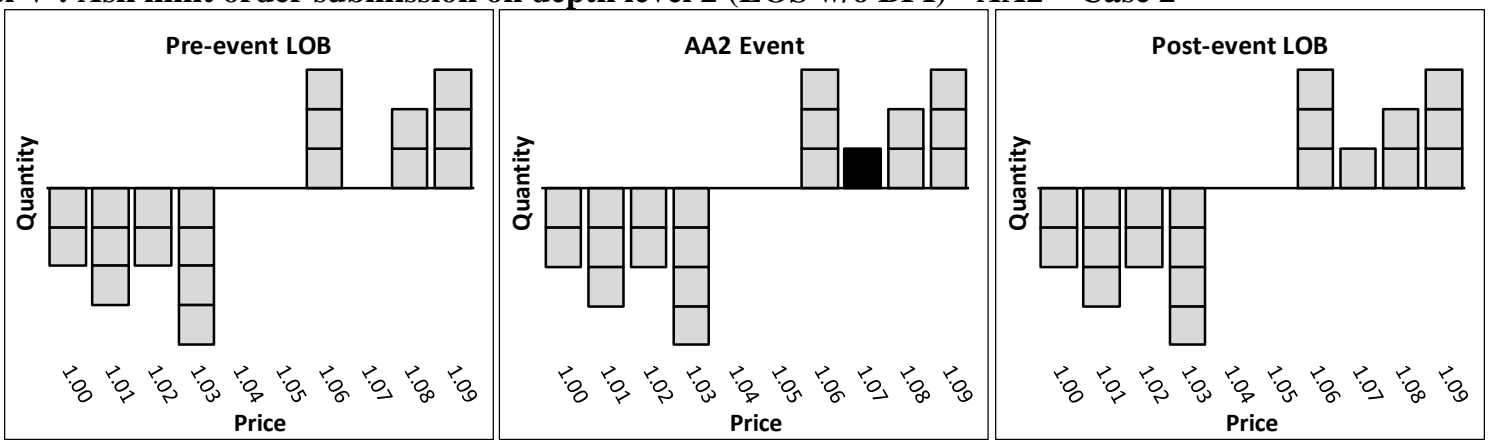

Panel VI : Ask limit order submission on depth level 2 (LOS w/o BPI) - AA2 - Case 3

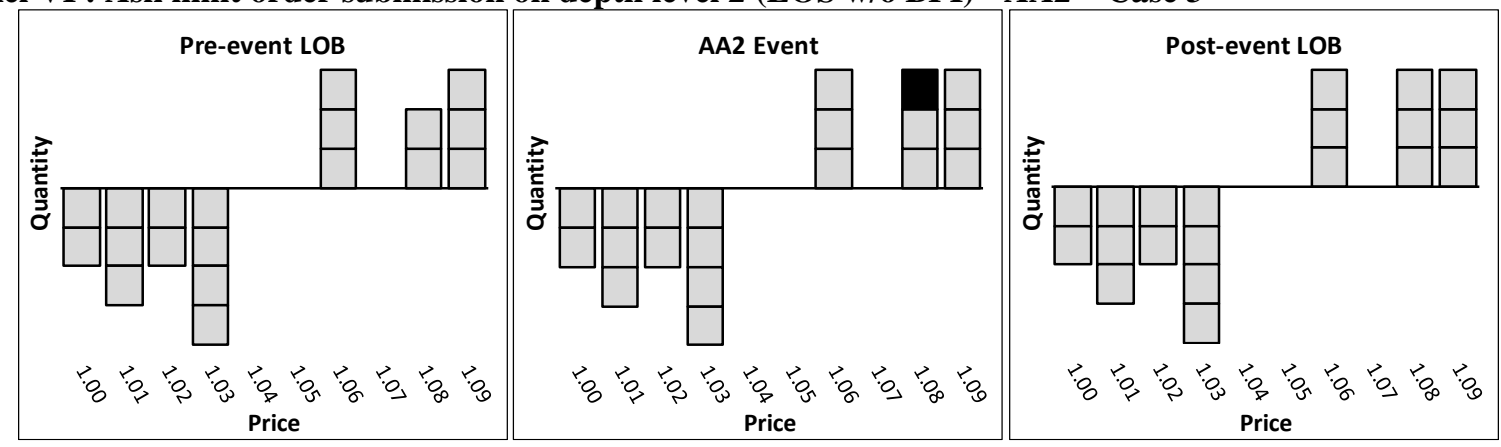

Panel VII : Bid limit order cancellation on depth level 1 (LOC) - BC1

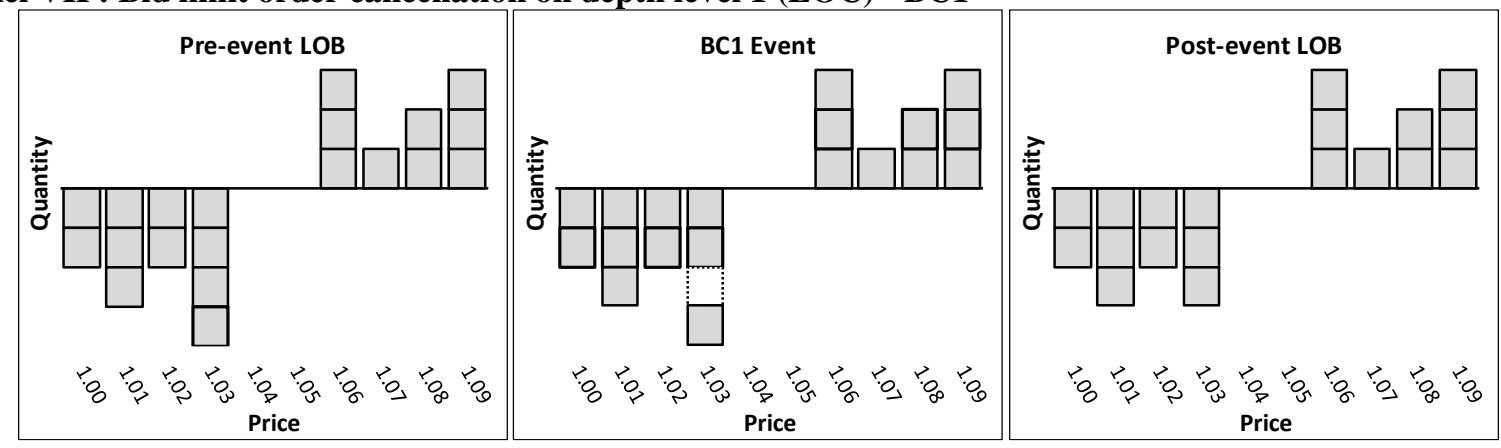


Figure 3 Events occurrences count daily averages

(a)
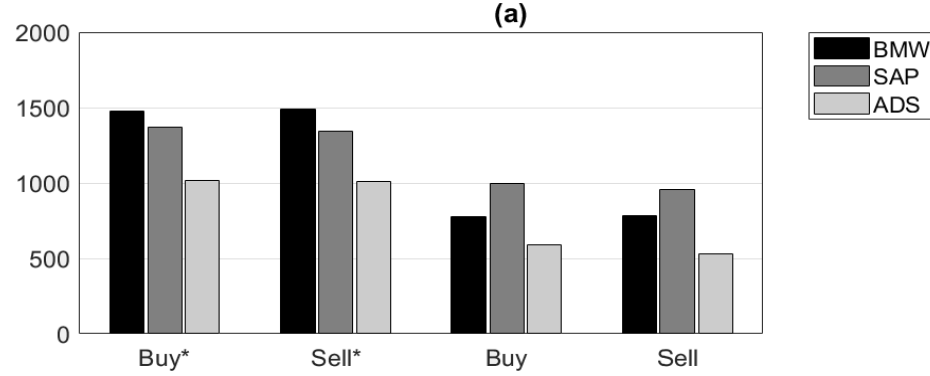

$\square$ ADS

(b)

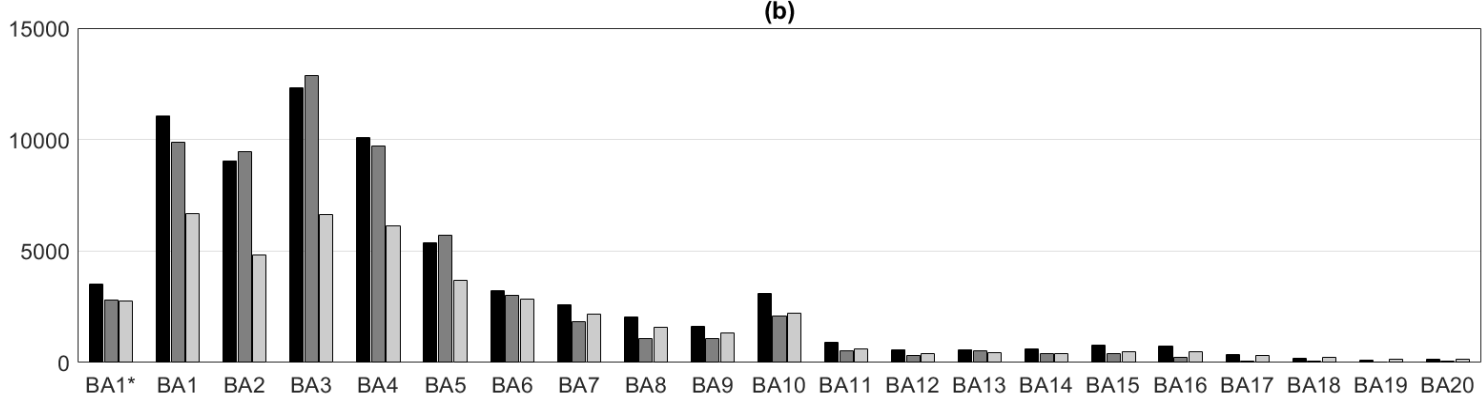

(c)

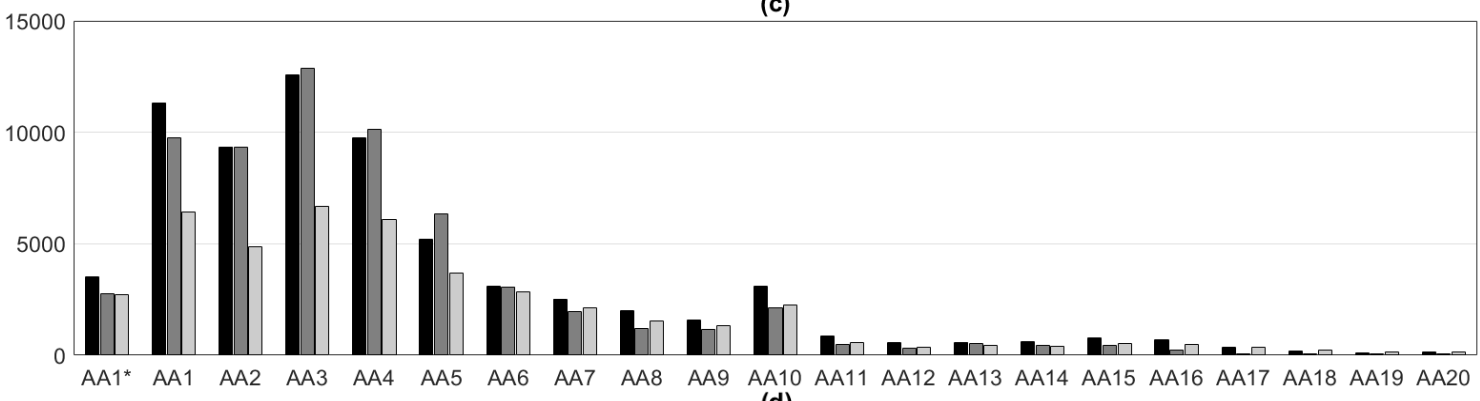

(d)

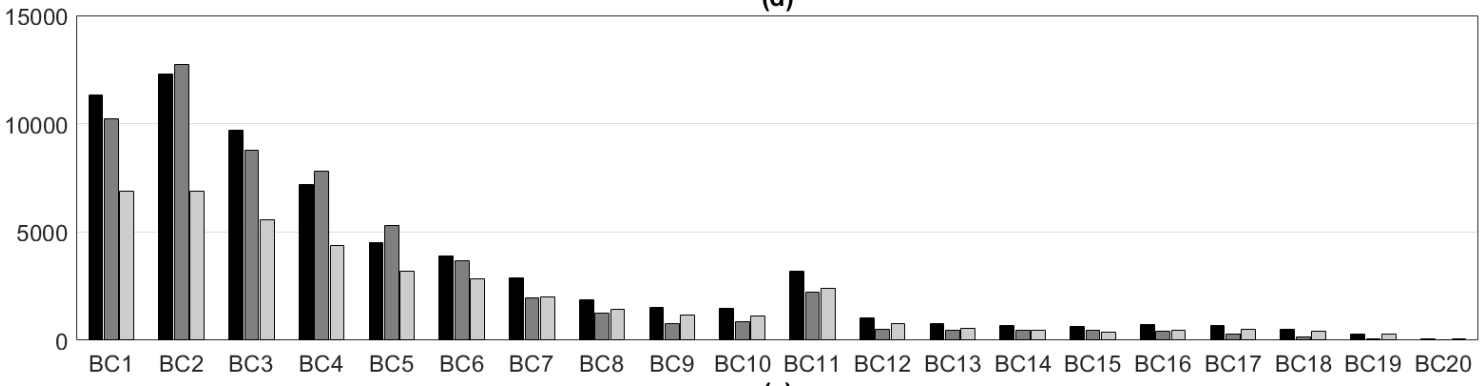

(e)

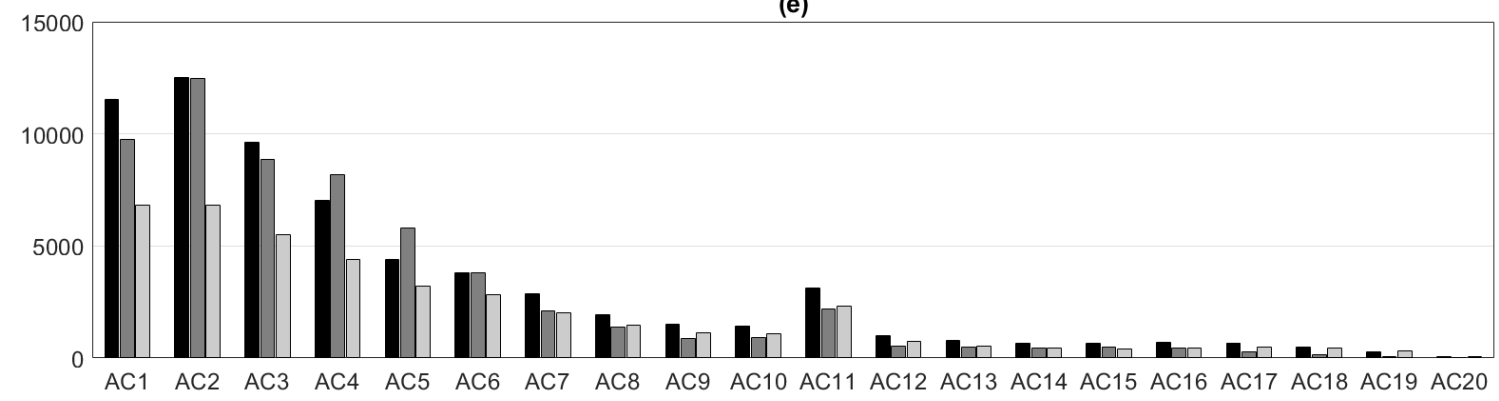




\section{Figure 4 Descriptive models Q-Q Plots}

Panel I: Trades w/ BPI, Trades w/o BPI and LOS w/ BPI

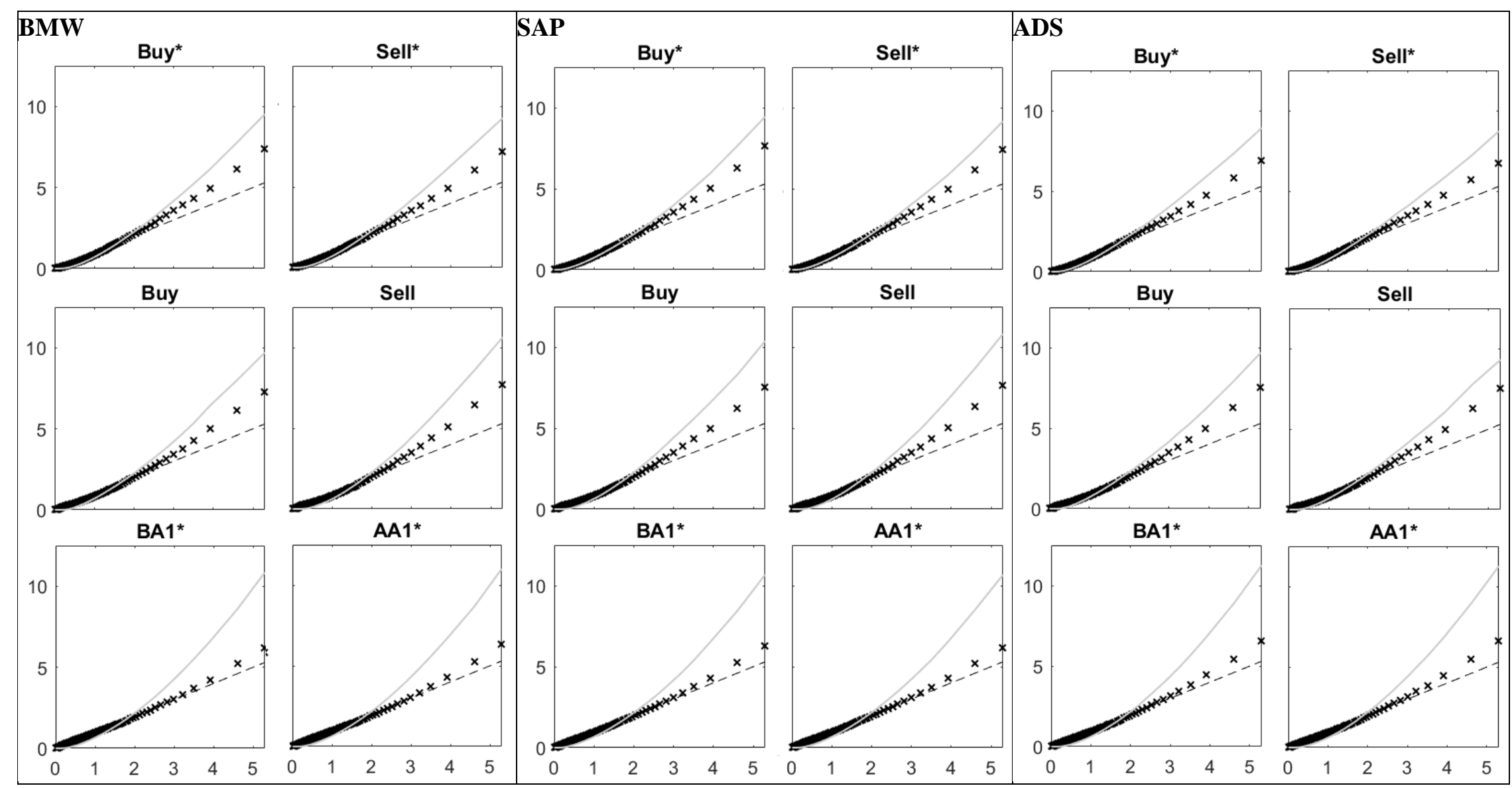


Panel II: Bid side LOS w/o BPI

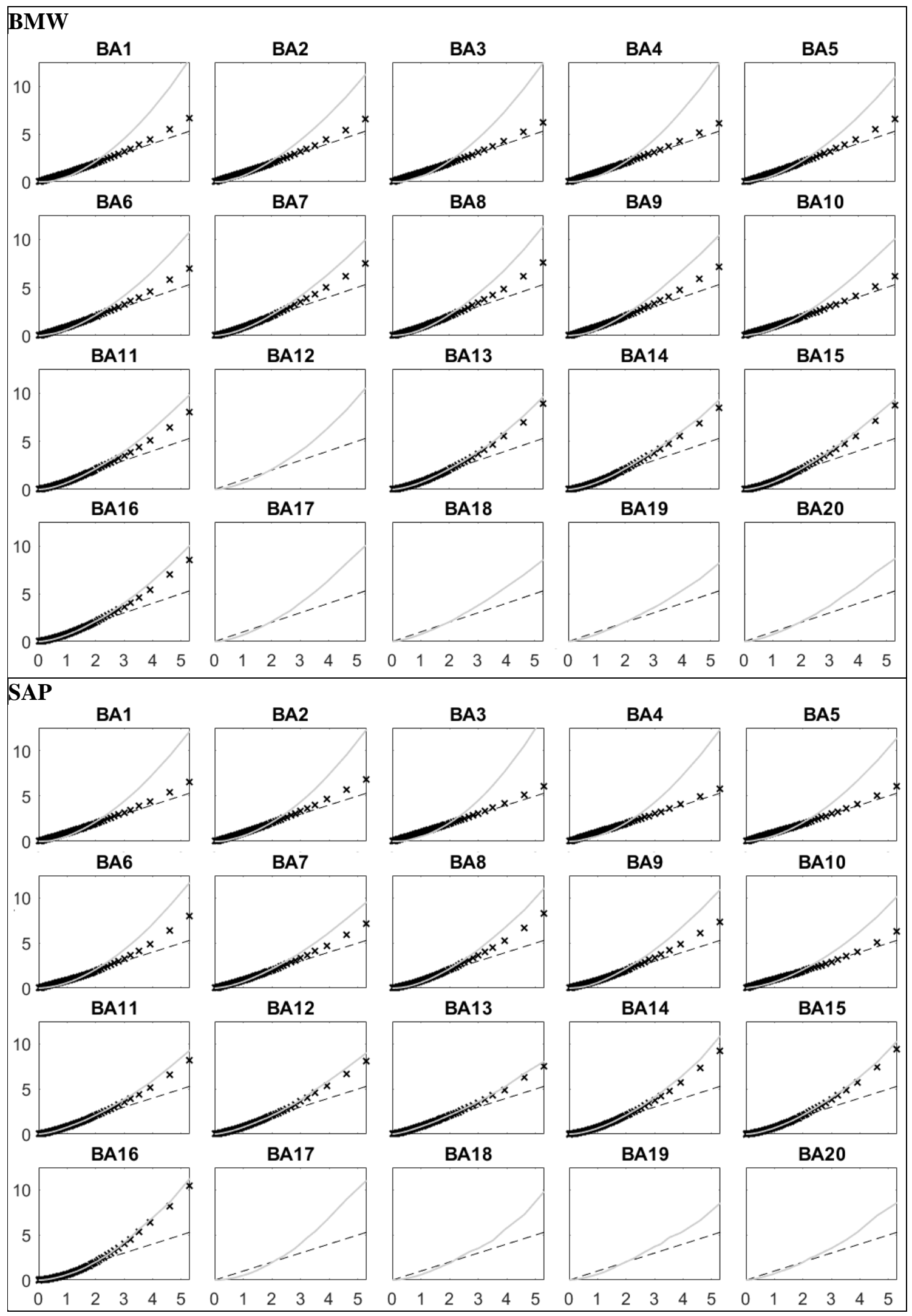




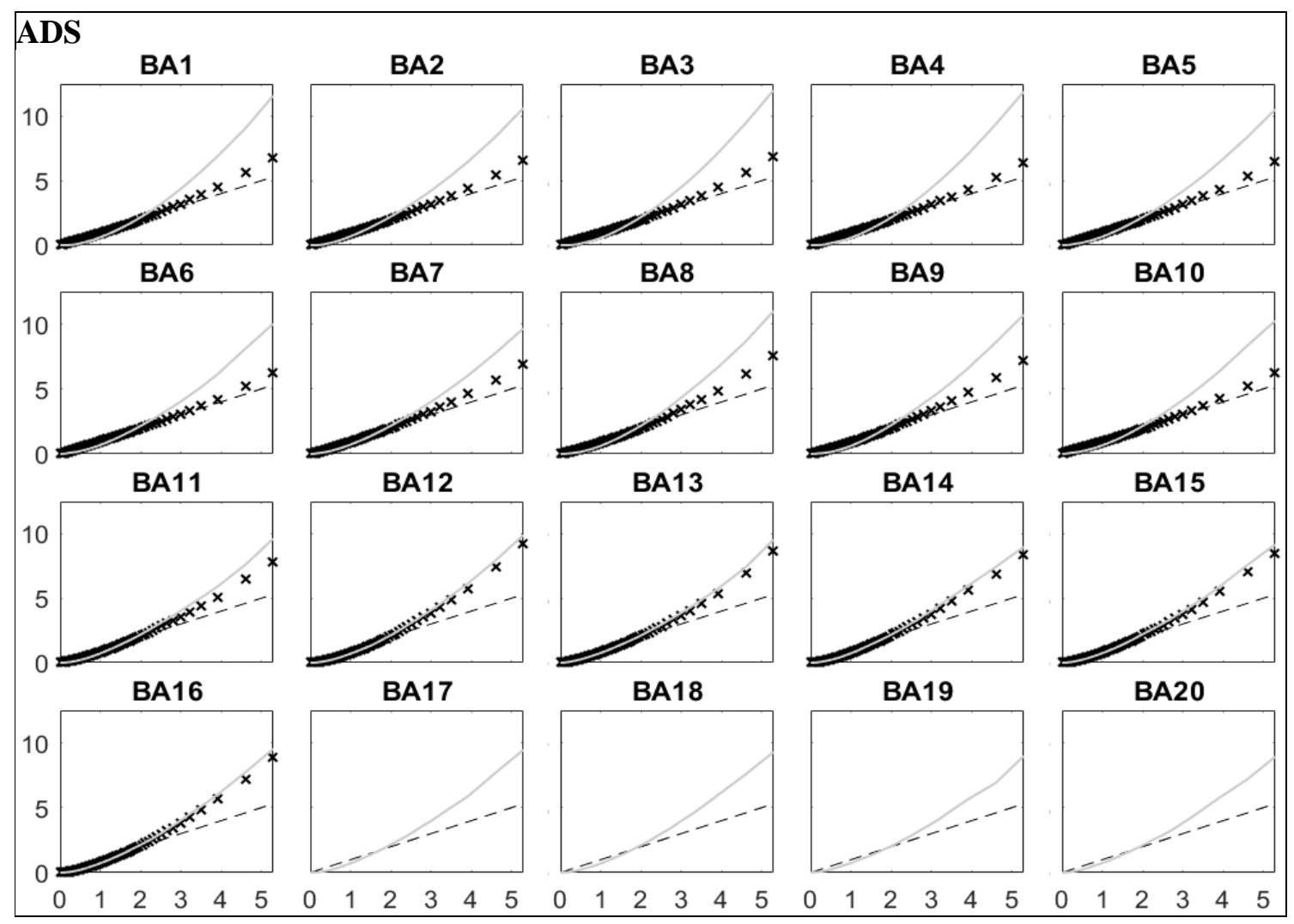

Panel III: Ask side LOS w/o BPI

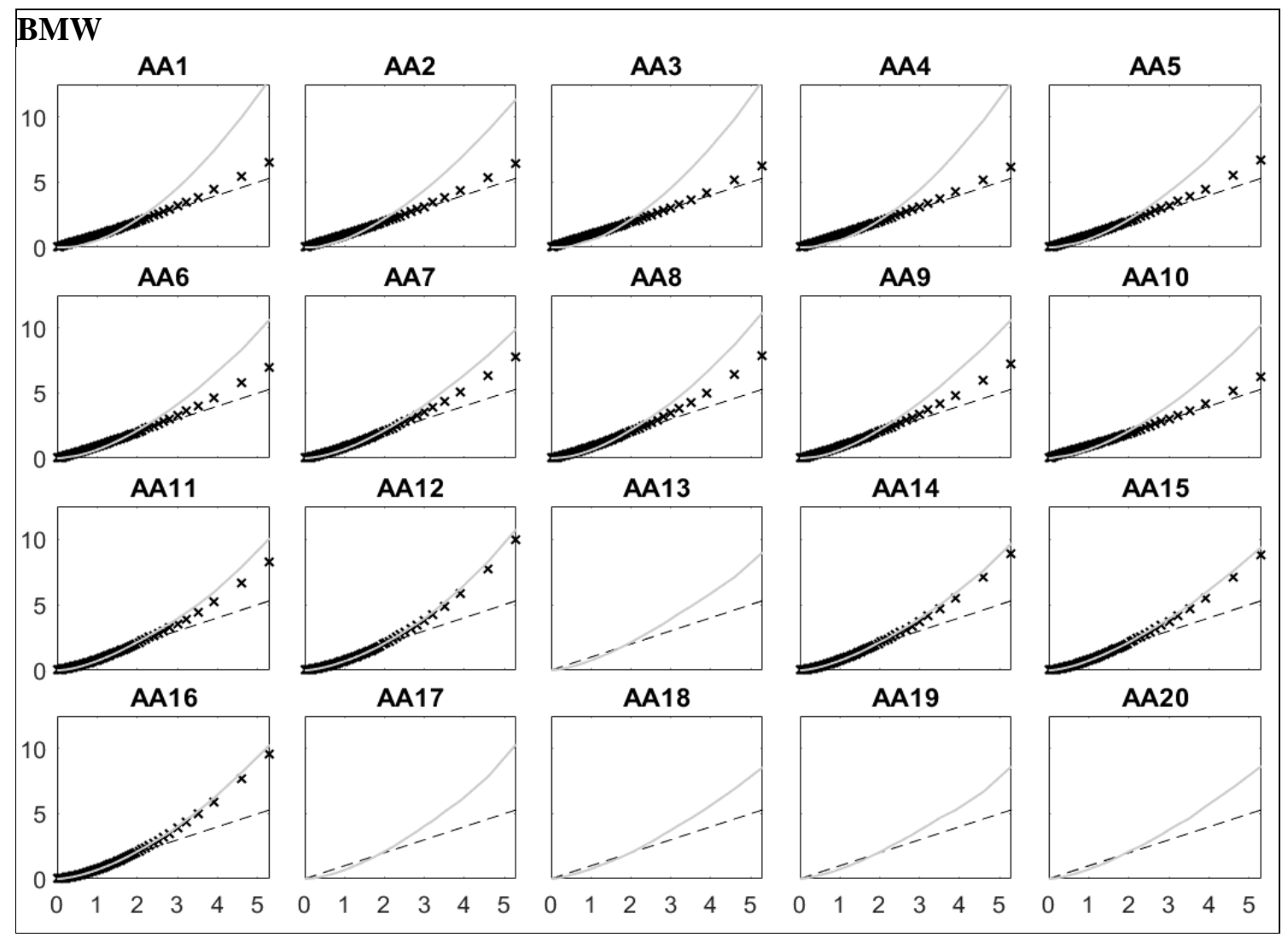




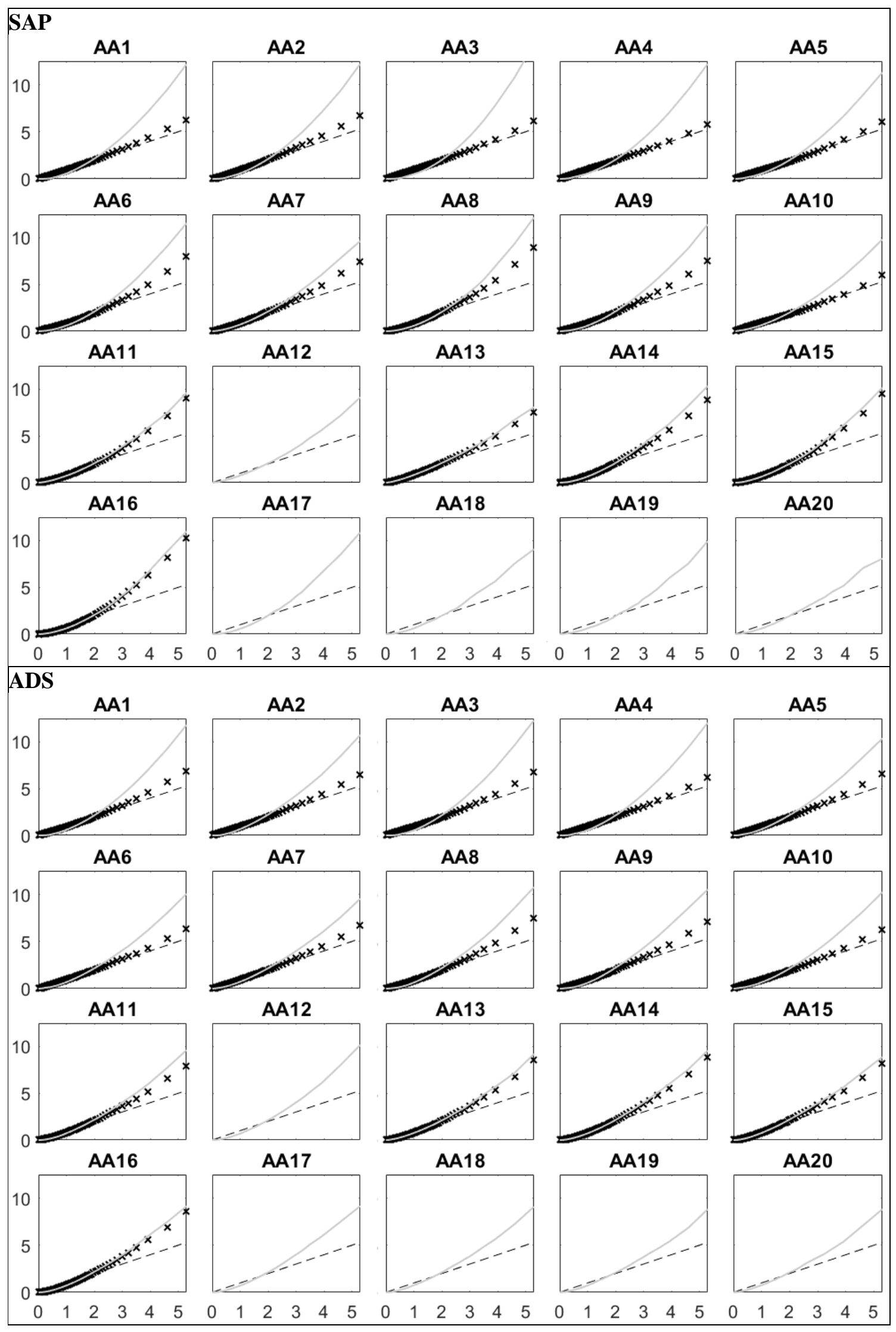


Panel IV: Bid side LOC
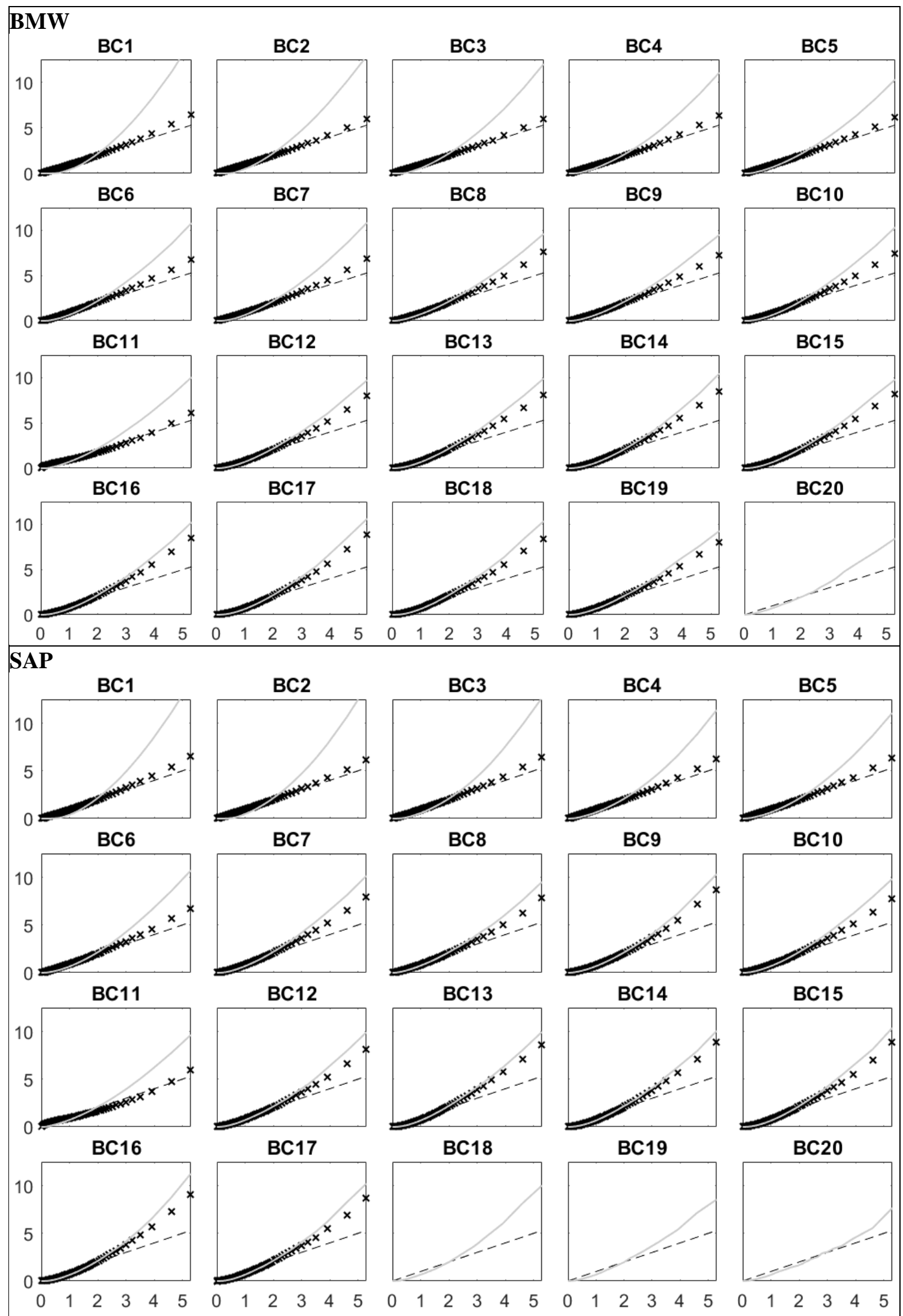


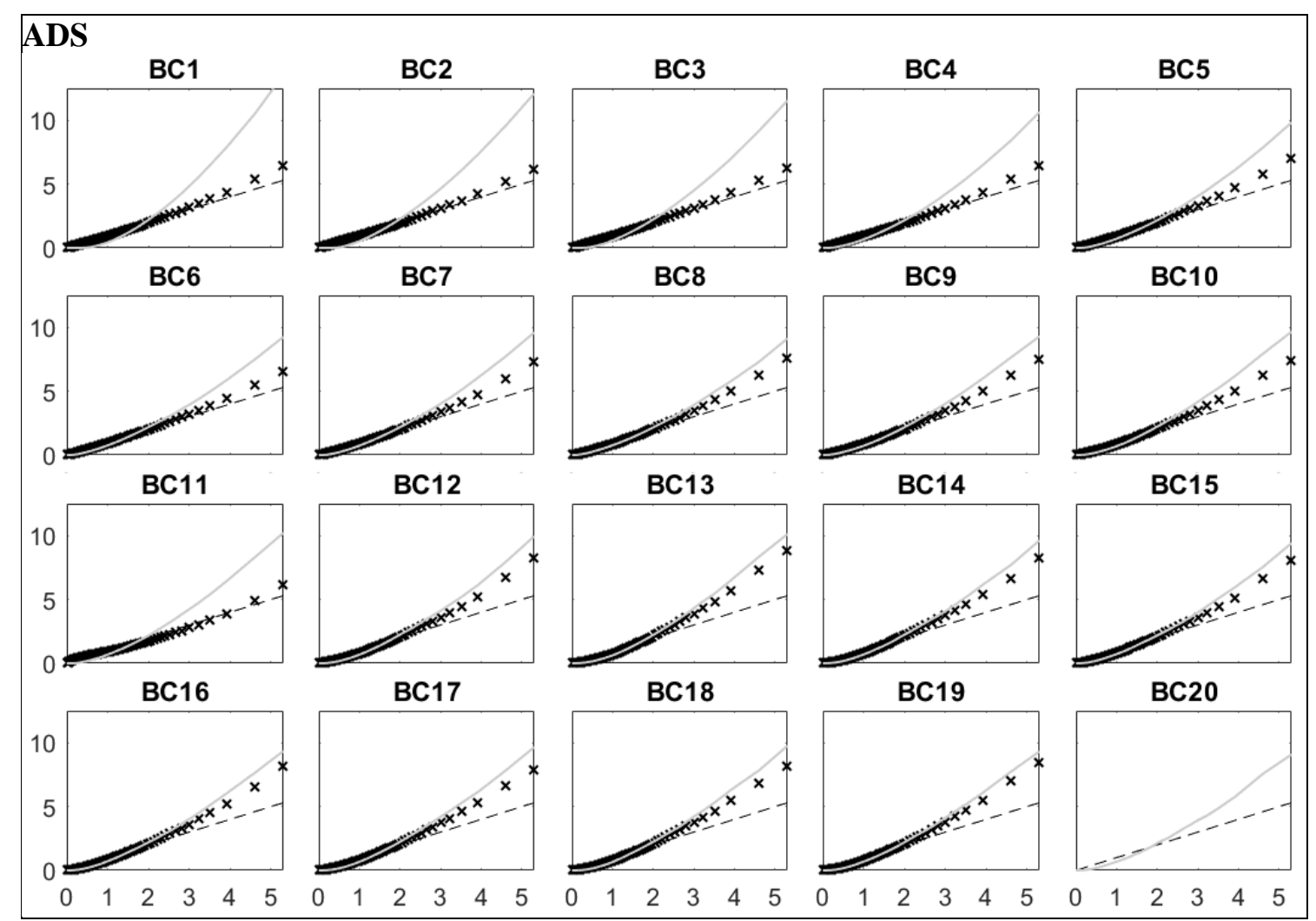

Panel V: Ask side LOC

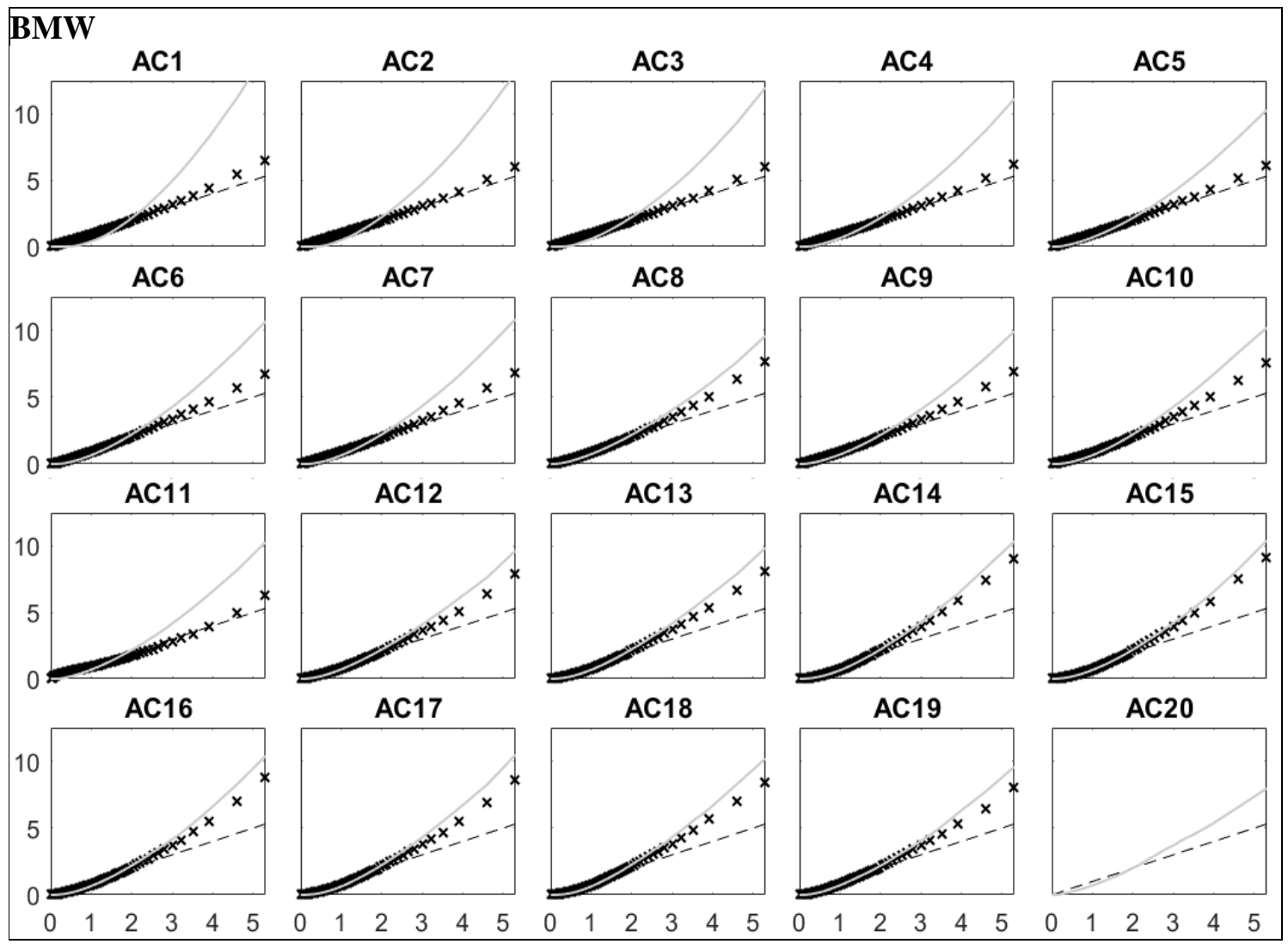




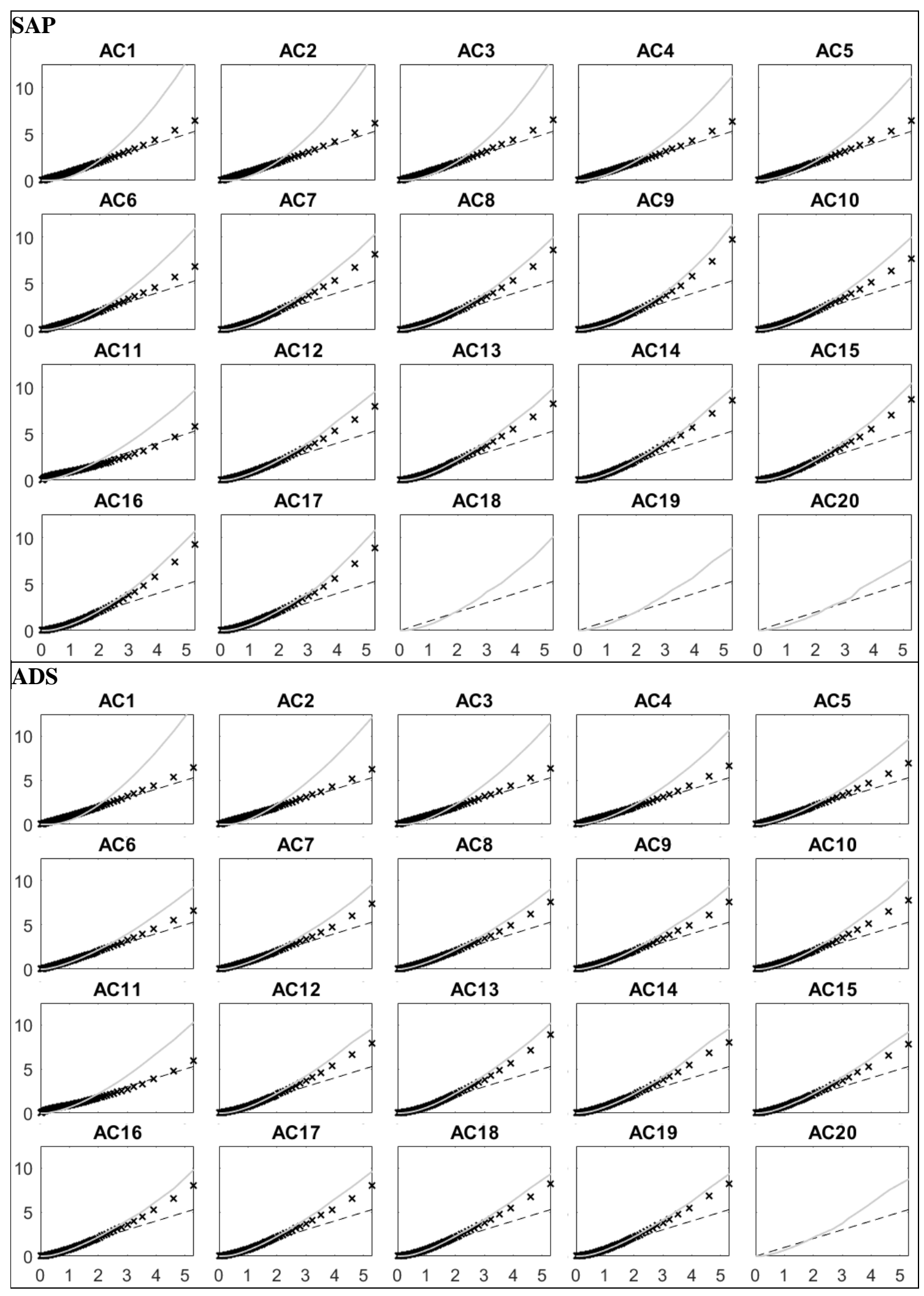


Figure 5 Events arrival process adjusted baseline daily average
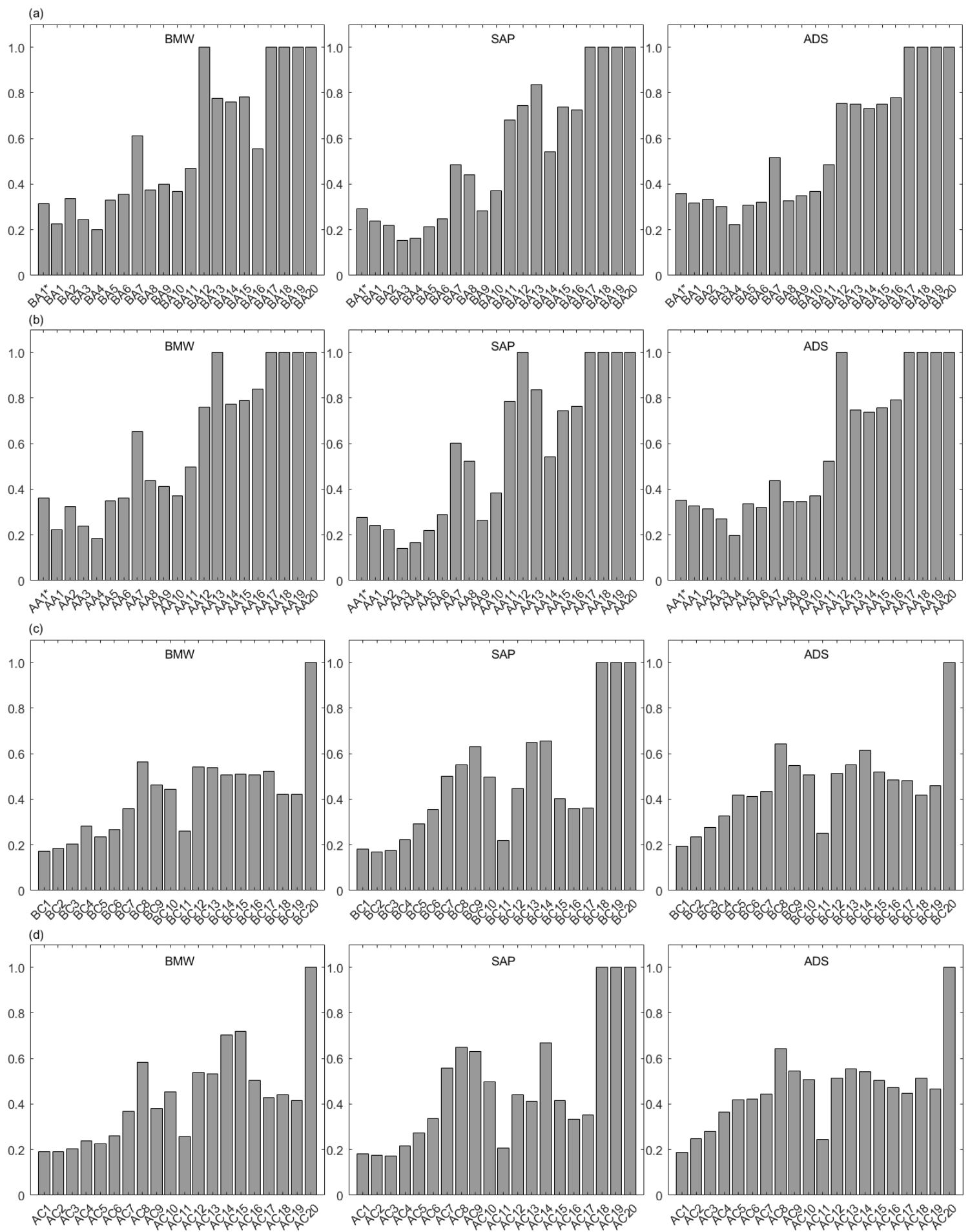


\section{Figure 6 Hawkes effects half-life daily average CDF}

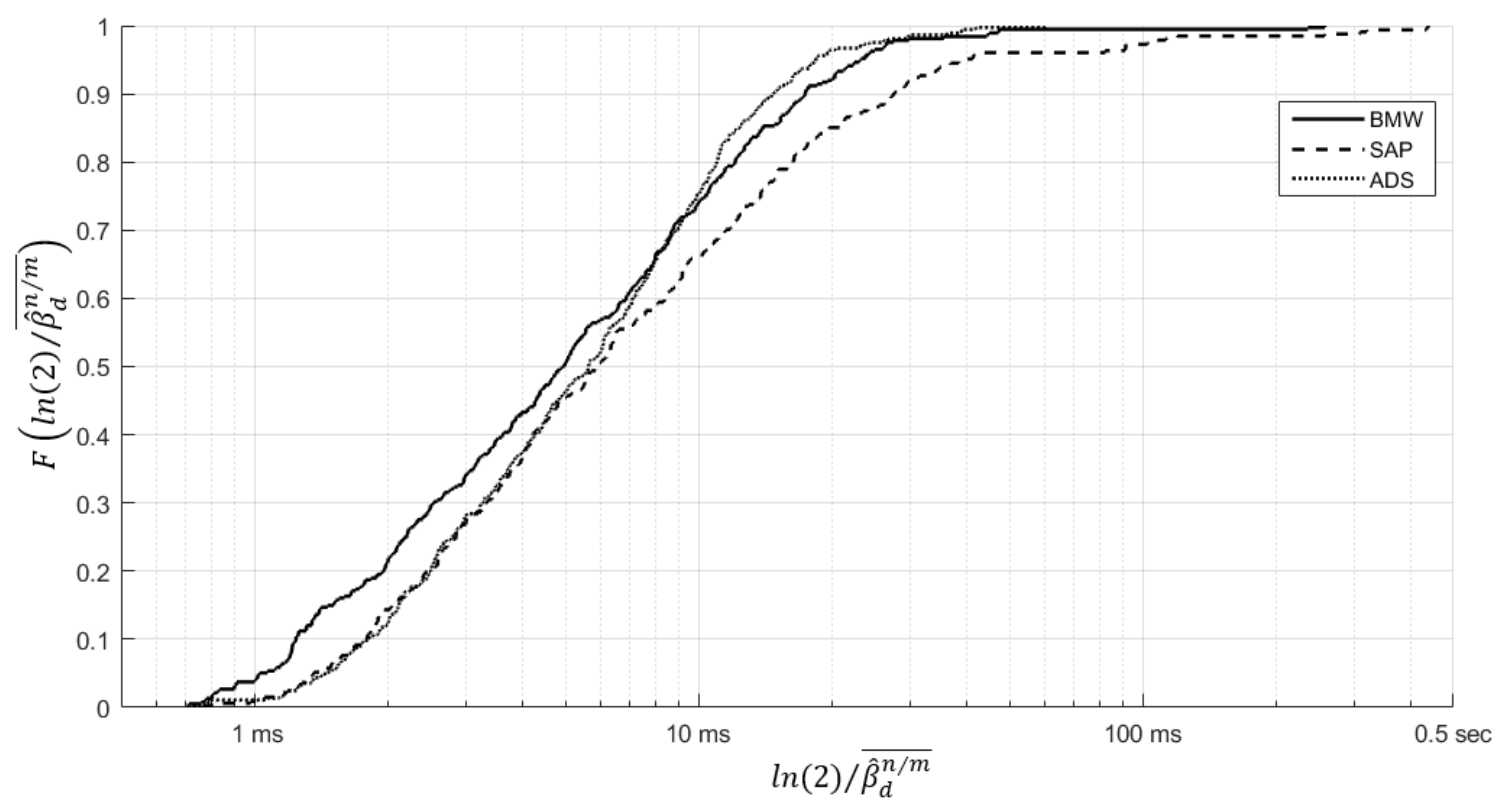

Figure 7 Hawkes effects Alpha parameters and Branching ratio with respect to Beta
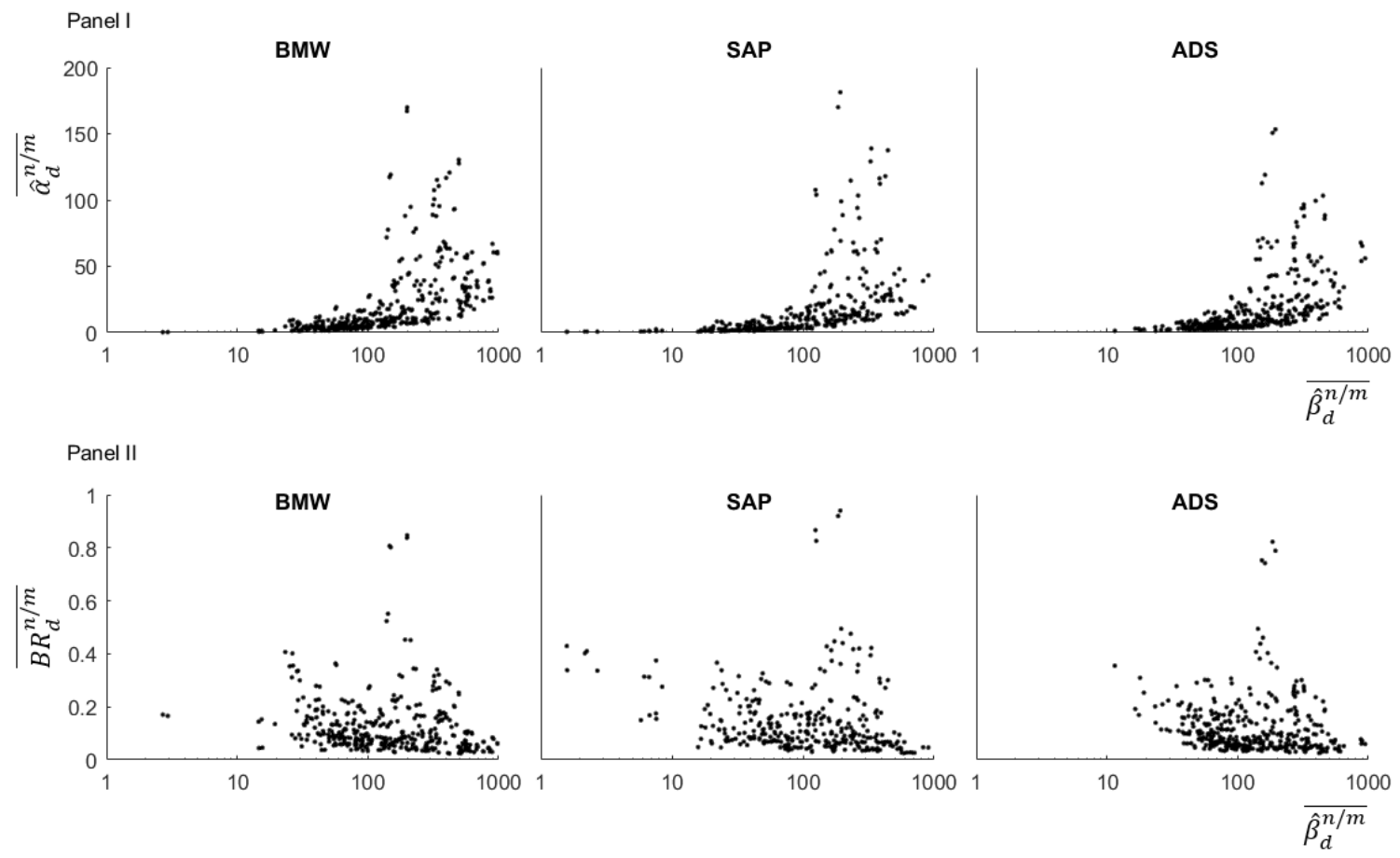

Panel I : $\hat{\alpha}_{d}^{n / m}$ daily average with respect to $\hat{\beta}_{d}^{n / m}$ daily average. Panel II : $B R_{d}^{n / m}$ daily average with respect to $\hat{\beta}_{d}^{n / m}$ daily average. 
Figure 8 Daily estimated parameters dispersion examples

Panel I: Buy*/BA1*
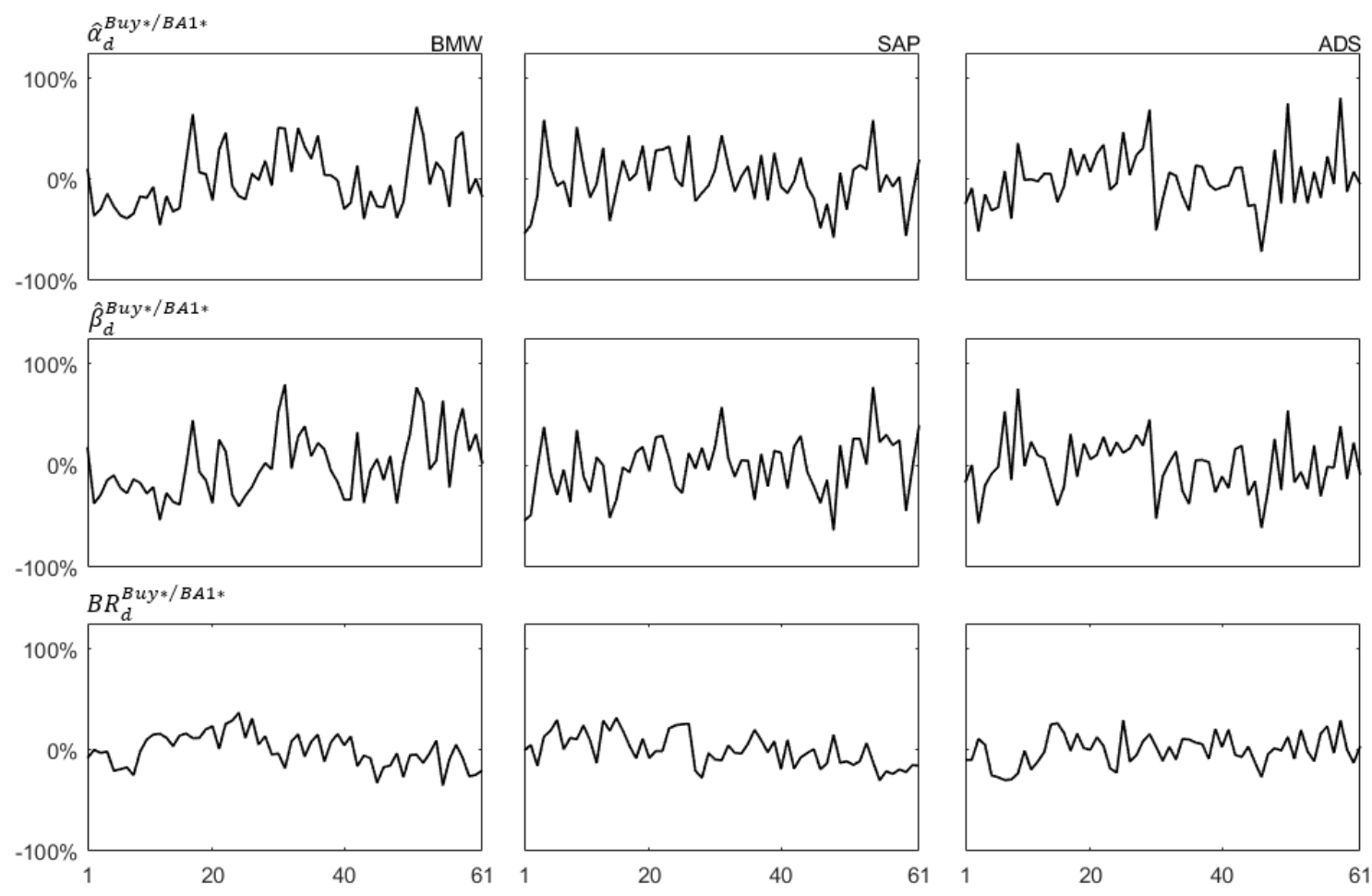

Panel II: Sell*/AA1*
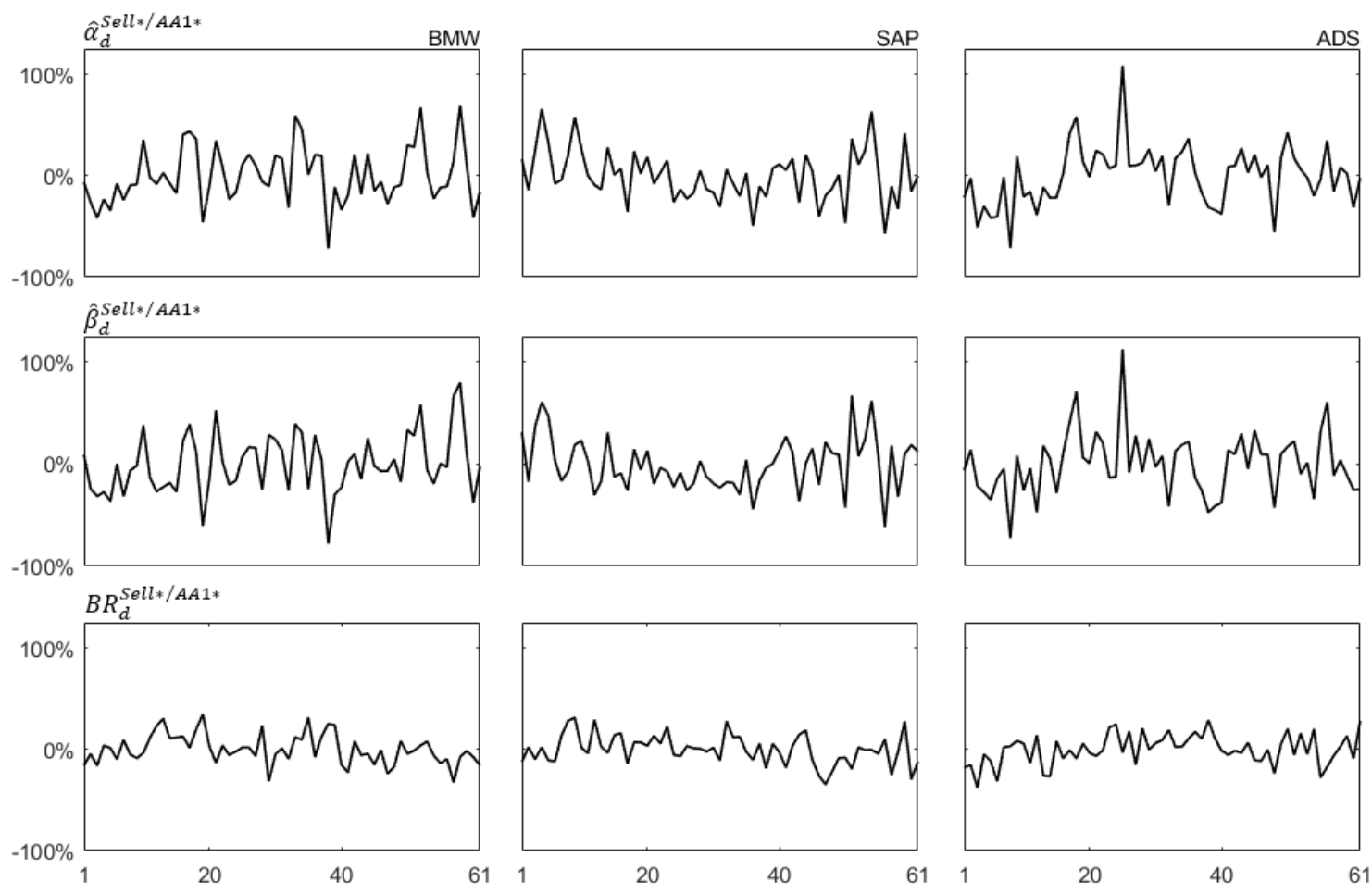


\section{Figure 9 General events dynamics}

Panel I: High-level dynamics

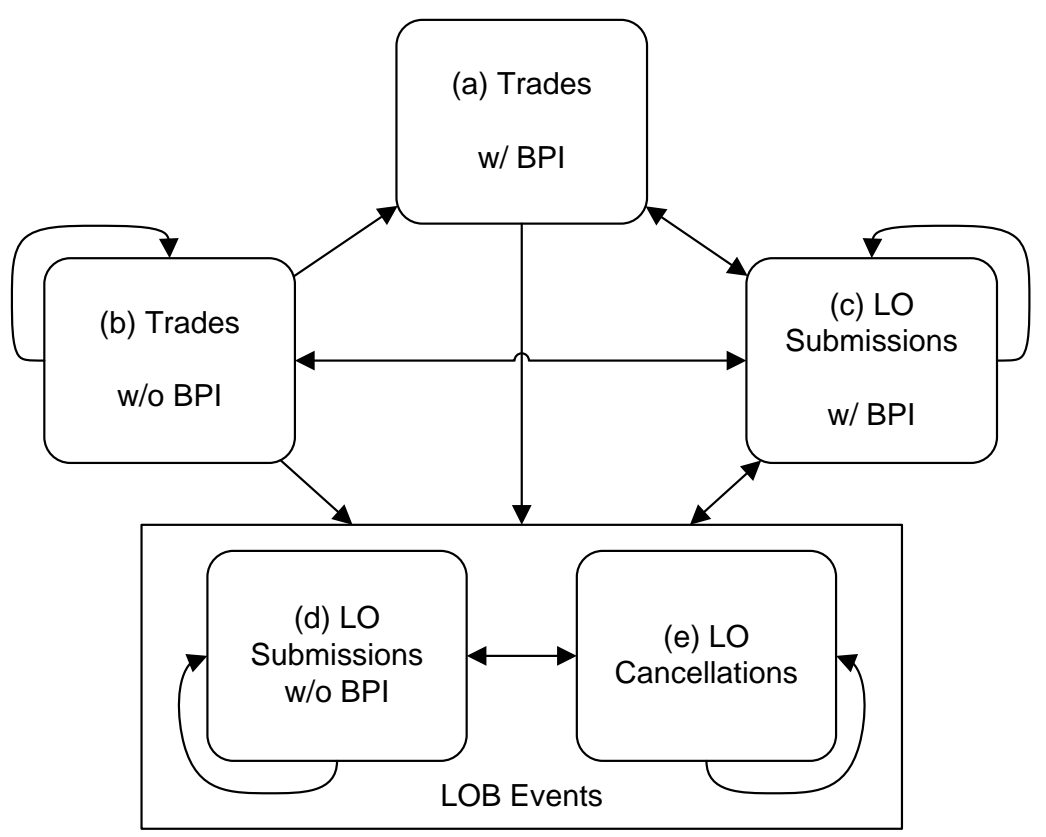

Panel II: Events relationships classes

\begin{tabular}{|c|c|c|c|c|c|c|c|c|c|c|}
\hline & Buy* & Sell* & Buy & Sell & BA1* & AA1* & $\begin{array}{l}\text { Bid LOS } \\
\text { w/o BPI }\end{array}$ & $\begin{array}{l}\text { Ask LOS } \\
\text { w/o BPI }\end{array}$ & Bid LOC & Ask LOC \\
\hline Buy* & & & & & E1 & E2 & $\mathrm{J} 1$ & $\mathrm{~J} 2$ & $\mathrm{~K} 1$ & $\mathrm{~K} 2$ \\
\hline \begin{tabular}{|l|} 
Sell* \\
\end{tabular} & & & & & E2 & E1 & $\mathrm{J} 2$ & $\mathrm{~J} 1$ & $\mathrm{~K} 2$ & $\mathrm{~K} 1$ \\
\hline Buy & A1 & & C1 & & F1 & & L1 & & & M1 \\
\hline Sell & & A1 & & $\mathrm{C} 1$ & & F1 & & L1 & M1 & \\
\hline \begin{tabular}{|l|} 
BA1* \\
\end{tabular} & B1 & $B 2$ & & $\mathrm{D} 1$ & $\mathrm{G} 1$ & & N1 & & 01 & $\mathrm{O} 2$ \\
\hline AA1* & B2 & B1 & D1 & & & $\mathrm{G} 1$ & & N1 & $\mathrm{O} 2$ & 01 \\
\hline \begin{tabular}{|l|} 
Bid \\
LOS \\
w/o \\
BPI \\
\end{tabular} & & & & & $\mathrm{H1}$ & & P1 & & Q1 & Q2 \\
\hline $\begin{array}{l}\text { Ask } \\
\text { LOS } \\
\text { w/o } \\
\text { BPI } \\
\end{array}$ & & & & & & $\mathrm{H} 1$ & & P1 & Q2 & Q1 \\
\hline $\begin{array}{l}\text { Bid } \\
\text { LOC }\end{array}$ & & & & & 11 & 12 & R1 & $\mathrm{R} 2$ & S1 & \\
\hline $\begin{array}{l}\text { Ask } \\
\text { LOC }\end{array}$ & & & & & 12 & 11 & $\mathrm{R} 2$ & R1 & & S1 \\
\hline
\end{tabular}


Panel III: Buyer side Trades w/ BPI, Trades w/o BPI and LOS w/ BPI

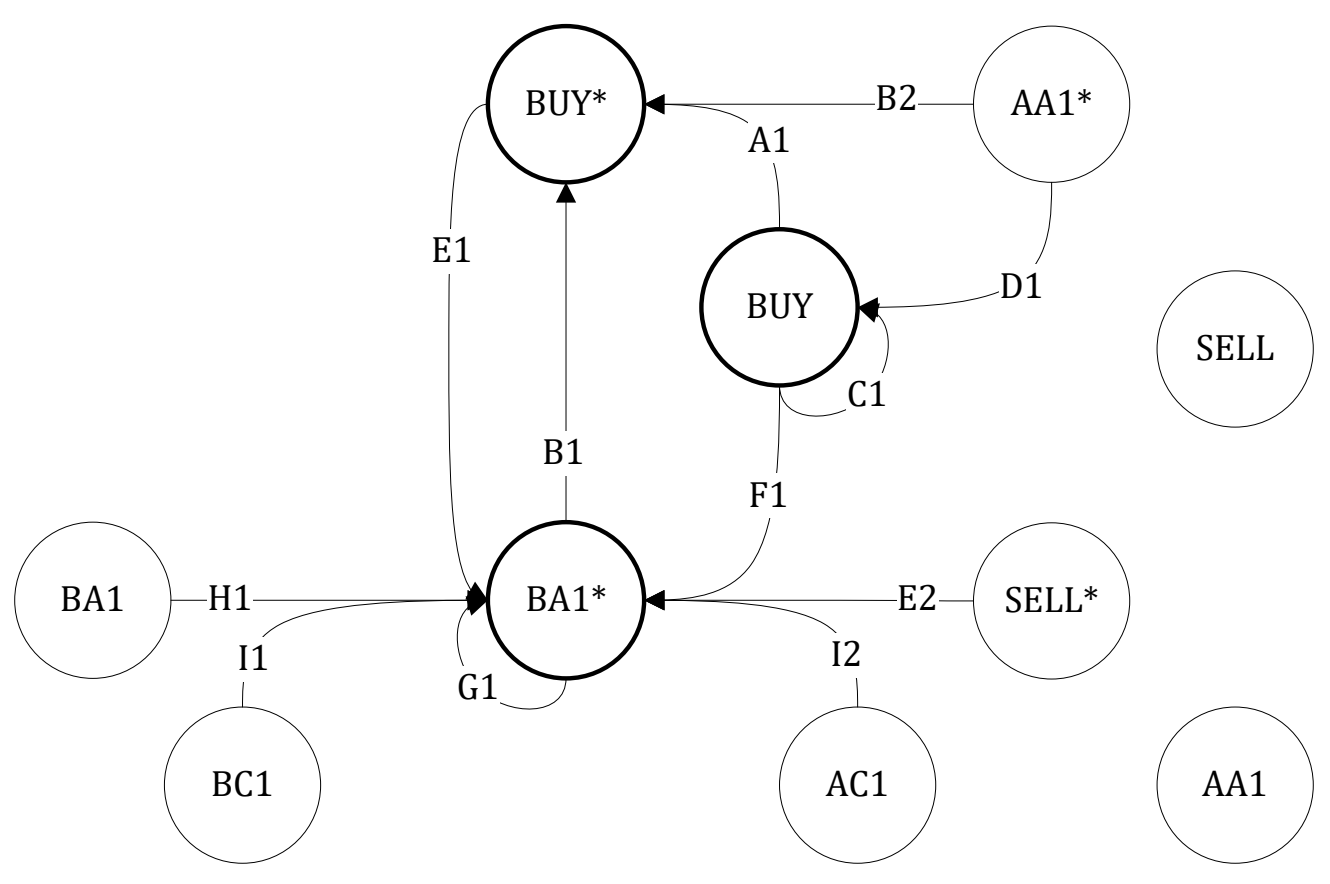

Panel IV: Buyer side LOS w/o BPI and LOC

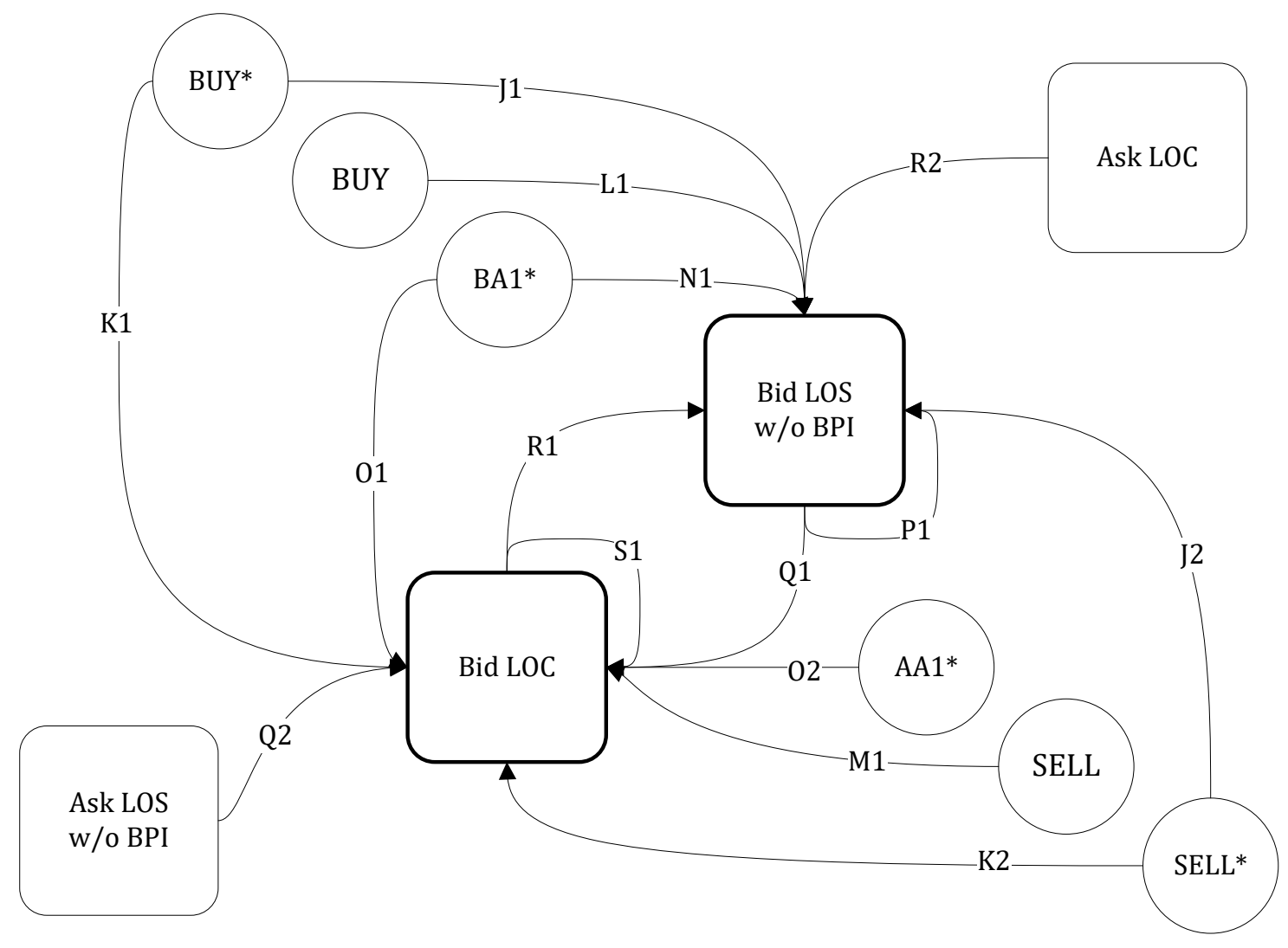


Figure 10 Trades and LOS w/ BPI effects branching ratio daily average

\section{Panel I: Buy*}
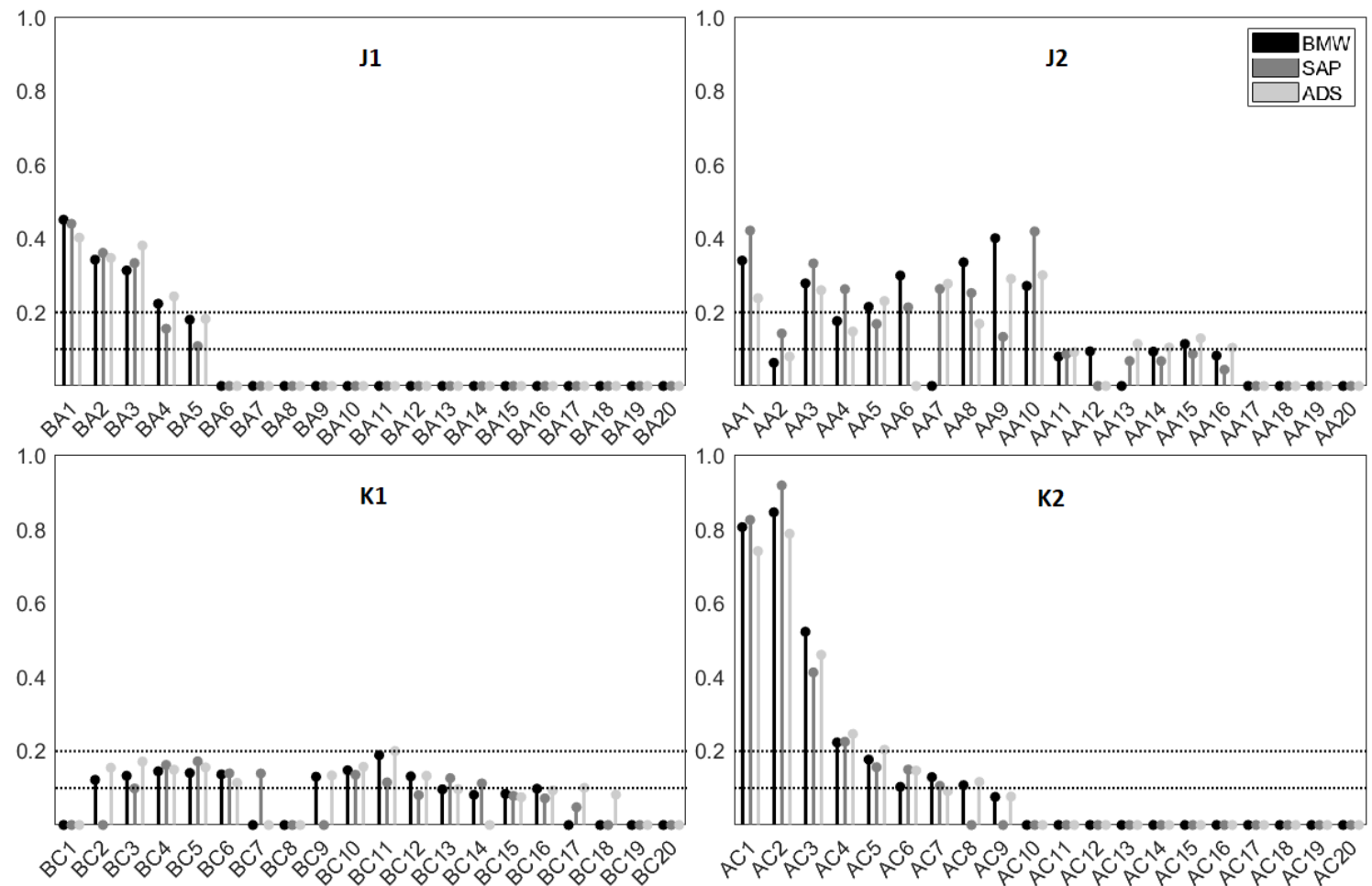

Panel II: Sell*
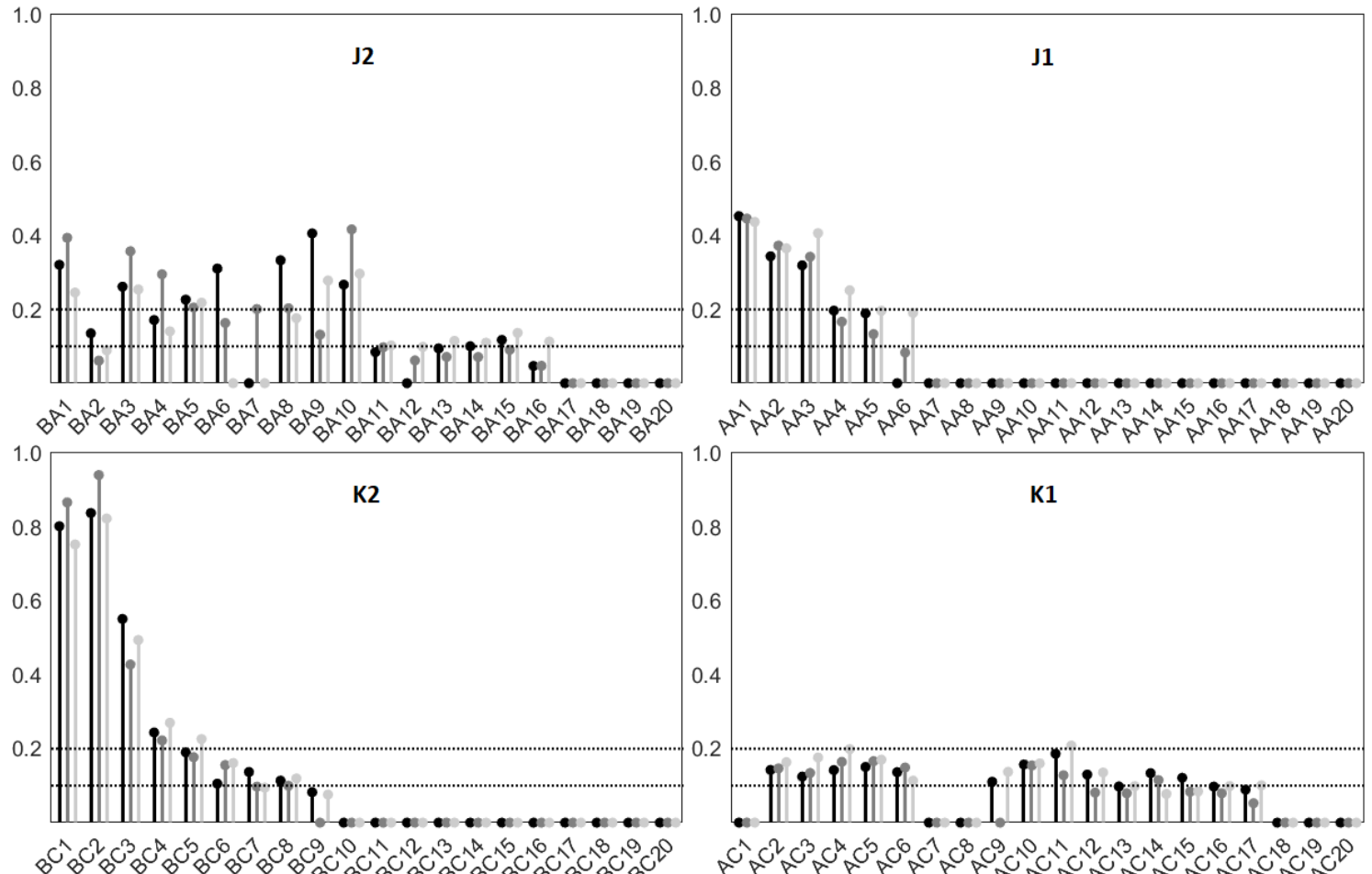


\section{Panel III: Buy}
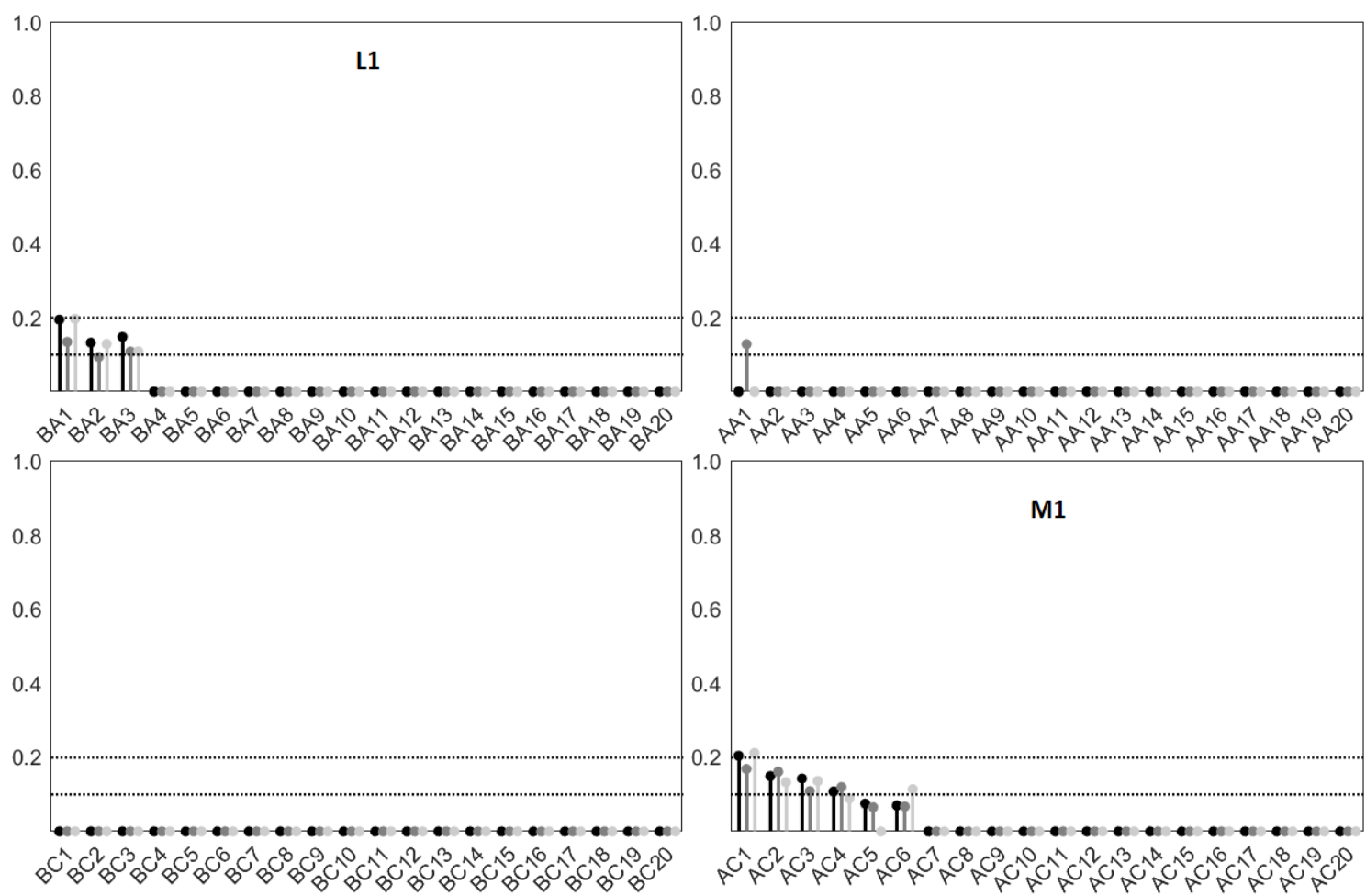

\section{Panel IV: Sell}
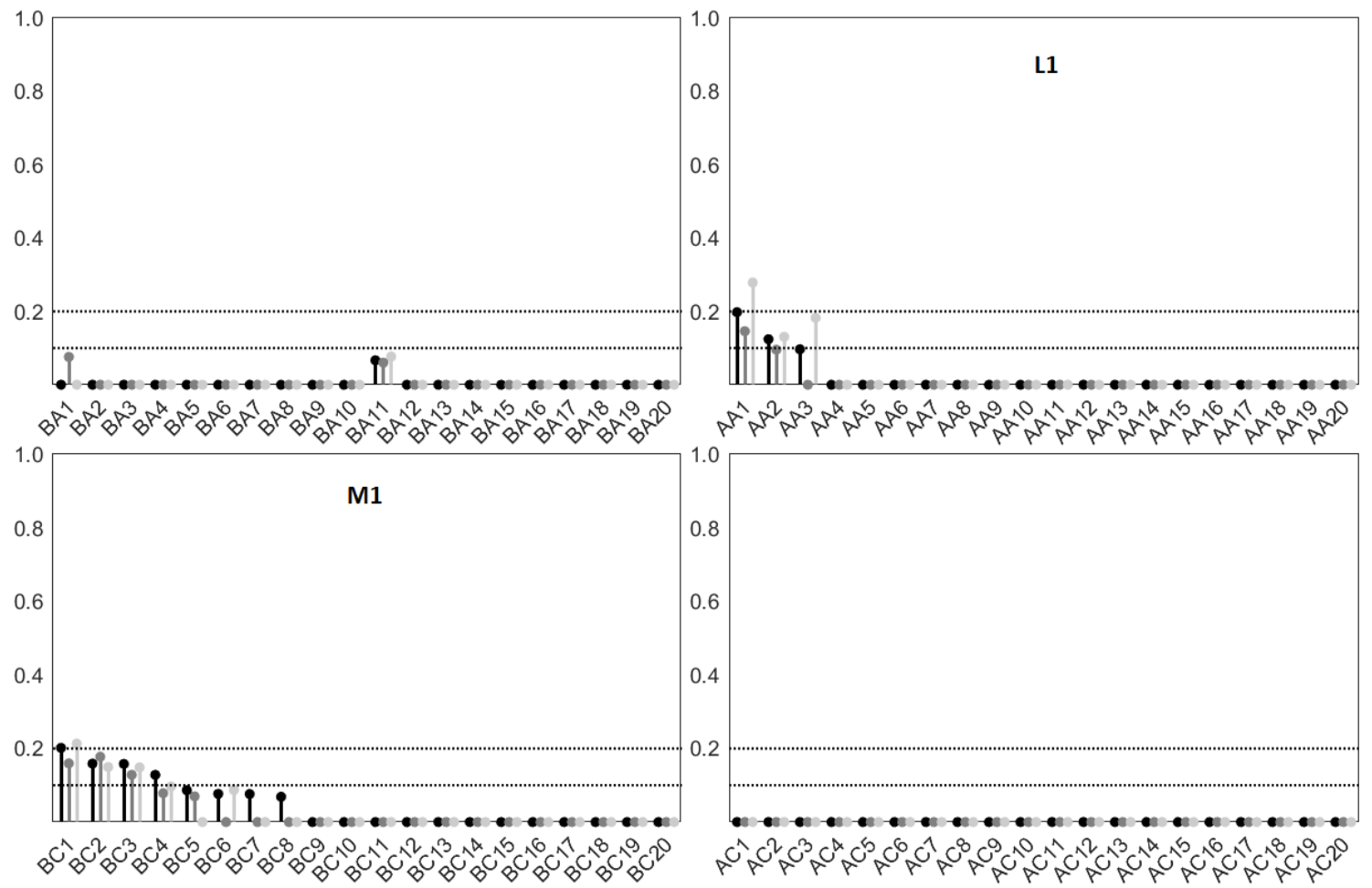


\section{Panel V: BA1*}
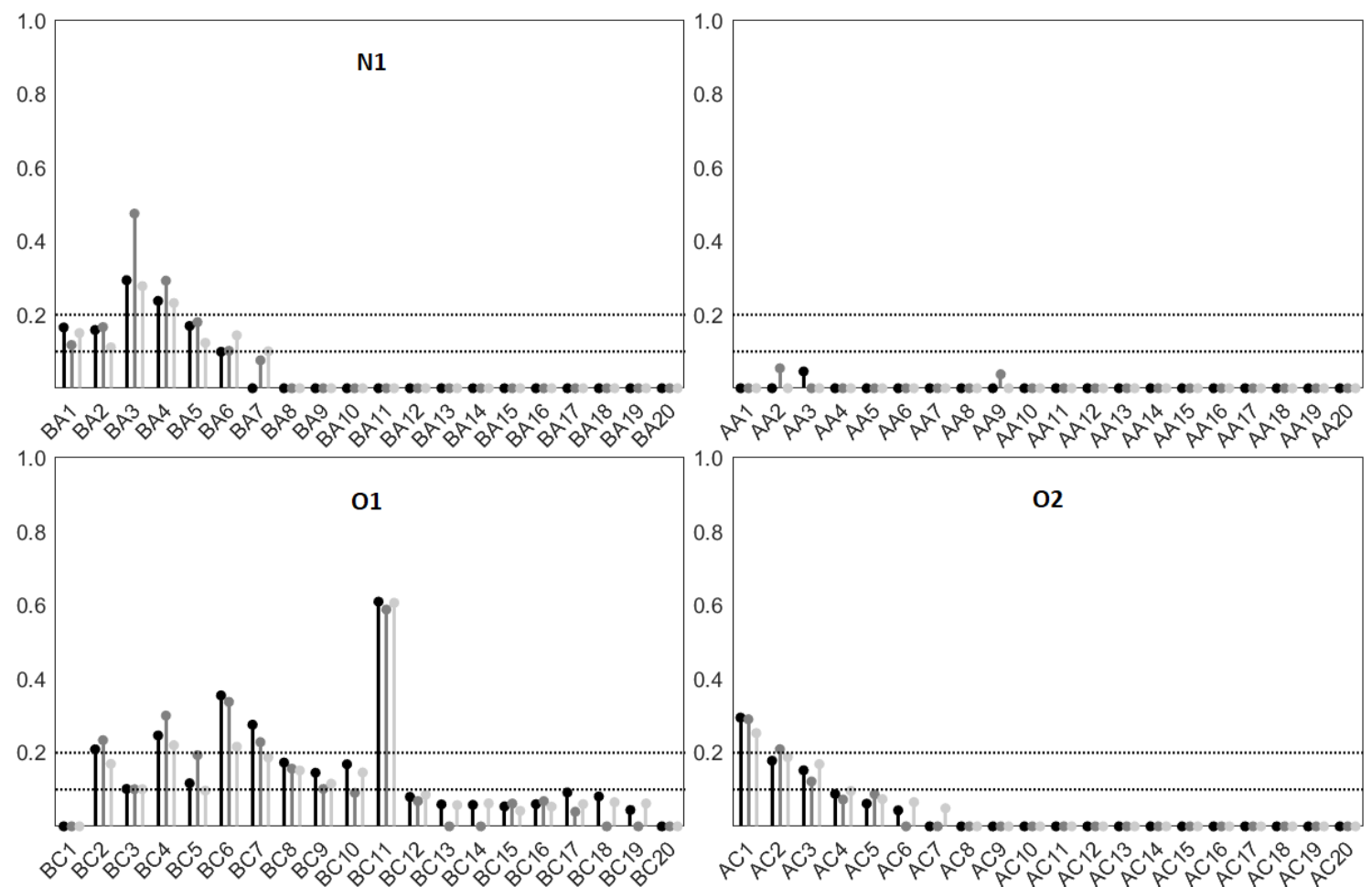

\section{Panel VI: AA1*}
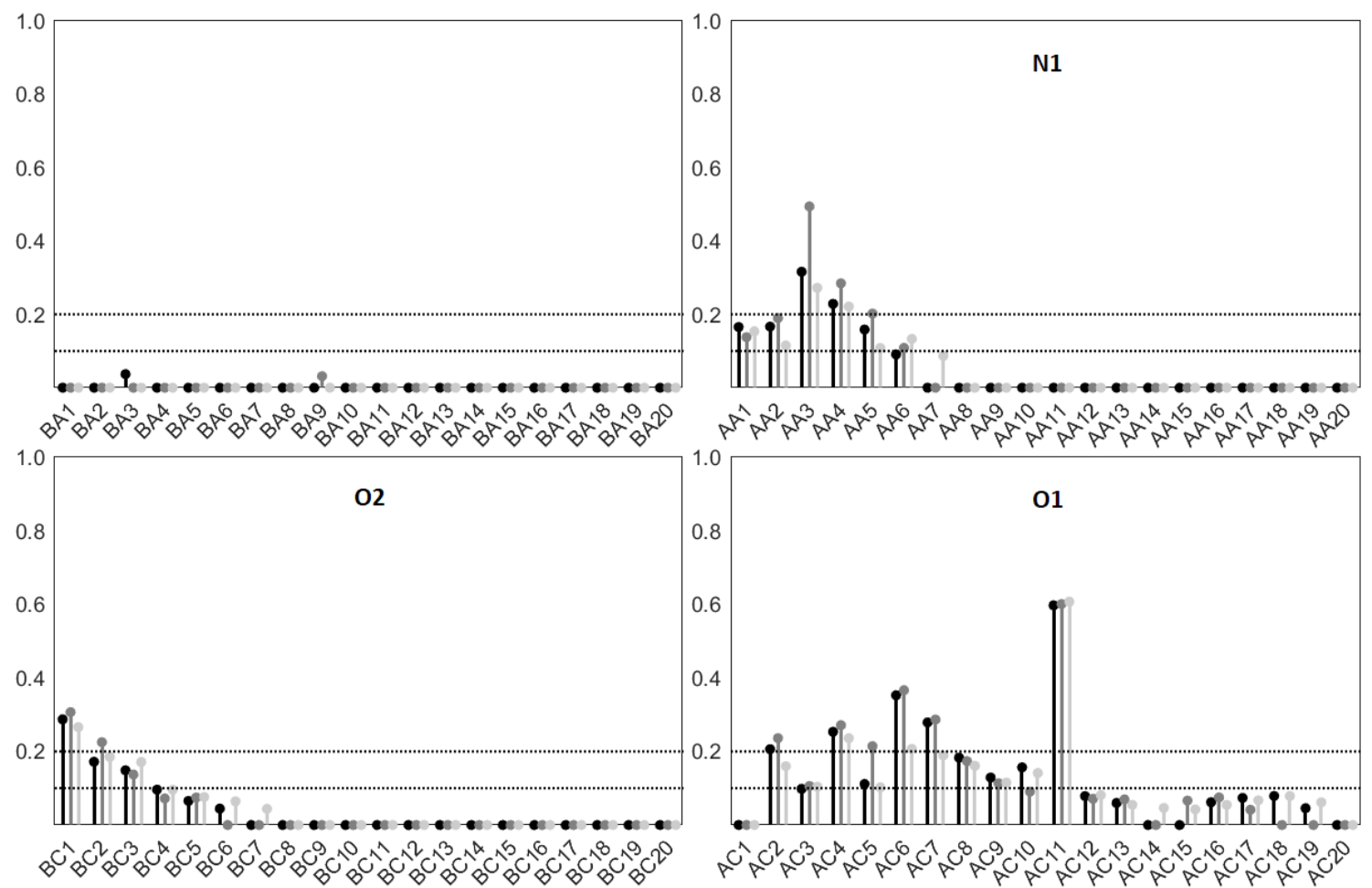
Figure 11 Category aggregated Hawkes effects contributions to LOB events arrival daily average
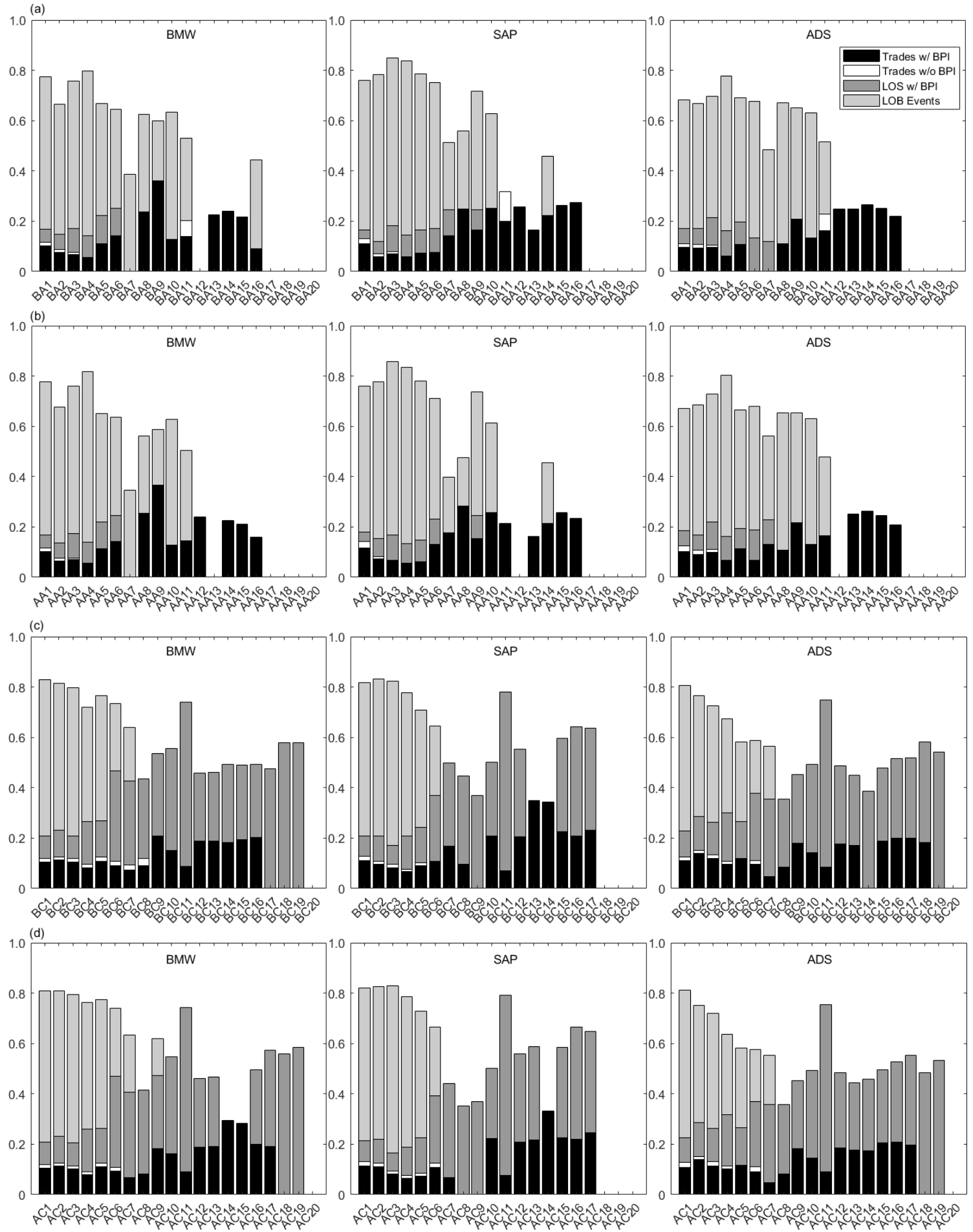
Table 1 Potential explanatory events sets definition

\begin{tabular}{|c|c|c|c|c|c|c|c|c|c|c|c|c|c|c|c|c|c|c|c|c|c|c|}
\hline & \multicolumn{2}{|c|}{$\begin{array}{l}\text { Trades } \\
\mathrm{w} / \mathrm{BPI}\end{array}$} & \multicolumn{2}{|c|}{$\begin{array}{l}\text { Trades } \\
\text { w/o BPI }\end{array}$} & \multicolumn{2}{|c|}{ LOS w/ BPI } & \multicolumn{4}{|c|}{ Bid LOS } & \multicolumn{4}{|c|}{ Ask LOS } & \multicolumn{4}{|c|}{ Bid LOC } & \multicolumn{4}{|c|}{ Ask LOC } \\
\hline & Buy* & Sell* & Buy & Sell & BA1* & AA1* & \begin{tabular}{|c|} 
BA1 \\
$\cdots$ \\
BA3
\end{tabular} & $\begin{array}{c}\text { BA4 } \\
\ldots \\
\text { BA6 }\end{array}$ & \begin{tabular}{|c|} 
BA7 \\
$\ldots$ \\
BA10 \\
\end{tabular} & $\begin{array}{c}\text { BA11 } \\
\ldots \\
\text { BA20 }\end{array}$ & \begin{tabular}{|c|}
$\mathrm{AA} 1$ \\
$\cdots$ \\
$\mathrm{AA} 3$
\end{tabular} & \begin{tabular}{|c|} 
AA4 \\
$\ldots$ \\
AA6
\end{tabular} & $\begin{array}{c}\text { AA7 } \\
\ldots \\
\text { AA10 }\end{array}$ & $\begin{array}{ll} & \text { AA11 } \\
\cdots & \\
0 \text { AA20 }\end{array}$ & $\begin{array}{c}\mathrm{BC1} \\
\ldots \\
\mathrm{BC3}\end{array}$ & $\begin{array}{c}\mathrm{BC4} \\
\cdots \\
\mathrm{BC} 6\end{array}$ & $\begin{array}{c}\mathrm{BC7} \\
\ldots \\
\mathrm{BC} 10\end{array}$ & \begin{tabular}{|c|c}
$\mathrm{BC} 11$ \\
$\ldots$ \\
0 \\
$\mathrm{BC} 20$ \\
\end{tabular} & \begin{tabular}{|c|}
$A C 1$ \\
$\cdots$ \\
$A C 3$
\end{tabular} & \begin{tabular}{|c|}
$\mathrm{AC4}$ \\
$\cdots$ \\
$\mathrm{AC6}$ \\
\end{tabular} & $\begin{array}{c}A C 7 \\
\ldots \\
A C 10\end{array}$ & $\begin{array}{c}\text { AC11 } \\
\cdots \\
\text { AC11 }\end{array}$ \\
\hline Buy* & $\checkmark$ & $\sqrt{ }$ & $\checkmark$ & $\checkmark$ & $\checkmark$ & $\checkmark$ & $\checkmark$ & $\checkmark$ & $\checkmark$ & $\checkmark$ & $\checkmark \mid$ & $\checkmark$ & $\checkmark$ & $\checkmark$ & $\checkmark$ & $\checkmark$ & $\checkmark$ & $\checkmark$ & $\checkmark$ & $\checkmark$ & $\checkmark$ & $\checkmark$ \\
\hline Sell* & $\checkmark$ & $\checkmark$ & $\checkmark$ & $\checkmark$ & $\checkmark$ & $\checkmark$ & $\checkmark$ & $\checkmark$ & $\checkmark$ & $\checkmark$ & $\checkmark$ & $\checkmark$ & $\checkmark$ & $\checkmark$ & $\checkmark$ & $\checkmark$ & $\checkmark$ & $\checkmark$ & $\checkmark$ & $\checkmark$ & $\checkmark$ & $\checkmark$ \\
\hline Buy & $\checkmark$ & $\checkmark$ & $\checkmark$ & $\checkmark$ & $\checkmark$ & $\checkmark$ & $\checkmark$ & $\checkmark$ & $\checkmark$ & $\checkmark$ & $\checkmark$ & $\checkmark$ & $\checkmark$ & $\checkmark$ & $\checkmark$ & $\checkmark$ & $\checkmark$ & $\checkmark$ & $\sqrt{ }$ & $\checkmark$ & $\checkmark$ & $\checkmark$ \\
\hline Sell & $\checkmark$ & $\checkmark$ & $\checkmark$ & $\checkmark$ & $\checkmark$ & $\checkmark$ & $\checkmark$ & $\checkmark$ & $\checkmark$ & $\checkmark$ & $\checkmark$ & $\checkmark$ & $\checkmark$ & $\checkmark$ & $\checkmark$ & $\checkmark$ & $\checkmark$ & $\checkmark$ & $\checkmark$ & $\checkmark$ & $\checkmark$ & $\checkmark$ \\
\hline BA1* & $\checkmark$ & $\checkmark$ & $\checkmark$ & $\checkmark$ & $\checkmark$ & $\sqrt{ }$ & $\sqrt{ }$ & $\checkmark$ & $\checkmark$ & $\sqrt{ }$ & $\checkmark$ & $\sqrt{ }$ & $\sqrt{ }$ & $\sqrt{ }$ & $\checkmark$ & $\sqrt{ }$ & $\sqrt{ }$ & $\checkmark$ & $\sqrt{ }$ & $\sqrt{ }$ & $\checkmark$ & $\sqrt{ }$ \\
\hline AA1* & $\checkmark$ & $\checkmark$ & $\checkmark$ & $\checkmark$ & $\checkmark$ & $\checkmark$ & $\checkmark$ & $\checkmark$ & $\checkmark$ & $\checkmark$ & $\checkmark$ & $\checkmark$ & $\checkmark$ & $\checkmark$ & $\checkmark$ & $\checkmark$ & $\checkmark$ & $\checkmark$ & $\checkmark$ & $\checkmark$ & $\checkmark$ & $\checkmark$ \\
\hline \begin{tabular}{|lll} 
BA1 & $\ldots$. & BA3 \\
\end{tabular} & $\checkmark$ & $\checkmark$ & $\checkmark$ & $\checkmark$ & $\checkmark$ & $\checkmark$ & $\checkmark$ & $\checkmark$ & $\checkmark$ & $\checkmark$ & $\checkmark$ & $\checkmark$ & $\checkmark$ & $\checkmark$ & $\checkmark$ & $\checkmark$ & $\checkmark$ & $\checkmark$ & $\checkmark$ & $\checkmark$ & $\checkmark$ & $\checkmark$ \\
\hline BA4 $\ldots$ BA6 & $\checkmark$ & $\checkmark$ & $\checkmark$ & $\checkmark$ & $\checkmark$ & $\checkmark$ & & $\checkmark$ & $\checkmark$ & $\checkmark$ & & $\checkmark$ & $\checkmark$ & $\checkmark$ & & $\checkmark$ & $\checkmark$ & $\checkmark$ & & $\checkmark$ & $\checkmark$ & $\checkmark$ \\
\hline BA7 $\quad \ldots$ BA10 & $\checkmark$ & $\checkmark$ & $\checkmark$ & $\checkmark$ & $\checkmark$ & $\checkmark$ & & & $\checkmark$ & $\checkmark$ & & & $\checkmark$ & $\checkmark$ & & & $\checkmark$ & $\checkmark$ & & & $\checkmark$ & $\checkmark$ \\
\hline BA11 ... BA20 & $\sqrt{ }$ & $\checkmark$ & $\checkmark$ & $\checkmark$ & $\checkmark$ & $\checkmark$ & & & & $\checkmark$ & & & & $\checkmark$ & & & & $\checkmark$ & & & & $\checkmark$ \\
\hline AA1 $\ldots$. AA3 & $\checkmark$ & $\checkmark$ & $\checkmark$ & $\checkmark$ & $\checkmark$ & $\checkmark$ & $\checkmark$ & $\checkmark$ & $\checkmark$ & $\checkmark$ & $\checkmark$ & $\checkmark$ & $\checkmark$ & $\checkmark$ & $\checkmark$ & $\checkmark$ & $\checkmark$ & $\checkmark$ & $\checkmark$ & $\checkmark$ & $\checkmark$ & $\checkmark$ \\
\hline AA4 $\ldots$ AA6 & $\checkmark$ & $\checkmark$ & $\checkmark$ & $\checkmark$ & $\checkmark$ & $\checkmark$ & & $\checkmark$ & $\checkmark$ & $\checkmark$ & & $\checkmark$ & $\checkmark$ & $\checkmark$ & & $\checkmark$ & $\checkmark$ & $\checkmark$ & & $\checkmark$ & $\checkmark$ & $\checkmark$ \\
\hline AA7 $\quad . .$. AA10 & $\checkmark$ & $\checkmark$ & $\checkmark$ & $\checkmark$ & $\checkmark$ & $\checkmark$ & & & $\checkmark$ & $\checkmark$ & & & $\checkmark$ & $\checkmark$ & & & $\checkmark$ & $\checkmark$ & & & $\checkmark$ & $\checkmark$ \\
\hline AA11 ... AA20 & $\checkmark$ & $\checkmark$ & $\checkmark$ & $\checkmark$ & $\checkmark$ & $\checkmark$ & & & & $\checkmark$ & & & & $\checkmark$ & & & & $\checkmark$ & & & & $\checkmark$ \\
\hline \begin{tabular}{|lll}
$\mathrm{BC} 1$ & $\ldots$ & $\mathrm{BC} 3$ \\
\end{tabular} & $\checkmark$ & $\sqrt{ }$ & $\checkmark$ & $\checkmark$ & $\checkmark$ & $\sqrt{ }$ & $\checkmark$ & $\checkmark$ & $\checkmark$ & $\sqrt{ }$ & $\checkmark$ & $\checkmark$ & $\checkmark$ & $\checkmark$ & $\sqrt{ }$ & $\checkmark$ & $\sqrt{ }$ & $\checkmark$ & $\checkmark$ & $\checkmark$ & $\checkmark$ & $\checkmark$ \\
\hline \begin{tabular}{|lll}
$\mathrm{BC} 4$ & $\ldots \mathrm{BC} 6$ \\
\end{tabular} & $\checkmark$ & $\checkmark$ & $\checkmark$ & $\checkmark$ & $\checkmark$ & $\checkmark$ & & $\checkmark$ & $\checkmark$ & $\checkmark$ & & $\checkmark$ & $\checkmark$ & $\checkmark$ & & $\checkmark$ & $\checkmark$ & $\checkmark$ & & $\checkmark$ & $\checkmark$ & $\checkmark$ \\
\hline $\begin{array}{lll}B C 7 & \ldots & B C 10 \\
\end{array}$ & $\checkmark$ & $\checkmark$ & $\checkmark$ & $\checkmark$ & $\checkmark$ & $\checkmark$ & & & $\checkmark$ & $\checkmark$ & & & $\checkmark$ & $\checkmark$ & & & $\checkmark$ & $\checkmark$ & & & $\checkmark$ & $\checkmark$ \\
\hline BC11 ... BC20 & $\checkmark$ & $\checkmark$ & $\checkmark$ & $\checkmark$ & $\checkmark$ & $\checkmark$ & & & & $\checkmark$ & & & & $\checkmark$ & & & & $\checkmark$ & & & & $\checkmark$ \\
\hline $\mathrm{AC1} 1 \ldots \mathrm{AC} 3$ & $\checkmark$ & $\checkmark$ & $\checkmark$ & $\checkmark$ & $\checkmark$ & $\checkmark$ & $\checkmark$ & $\checkmark$ & $\checkmark$ & $\checkmark$ & $\checkmark$ & $\checkmark$ & $\checkmark$ & $\checkmark$ & $\sqrt{ }$ & $\sqrt{ }$ & $\checkmark$ & $\checkmark$ & $\checkmark$ & $\checkmark$ & $\sqrt{ }$ & $\checkmark$ \\
\hline AC4 $\ldots$ AC6 & $\checkmark$ & $\checkmark$ & $\checkmark$ & $\checkmark$ & $\checkmark$ & $\checkmark$ & & $\checkmark$ & $\checkmark$ & $\checkmark$ & & $\checkmark$ & $\checkmark$ & $\checkmark$ & & $\checkmark$ & $\checkmark$ & $\checkmark$ & & $\checkmark$ & $\checkmark$ & $\checkmark$ \\
\hline AC7 $\ldots$ AC10 & $\checkmark$ & $\checkmark$ & $\checkmark$ & $\checkmark$ & $\checkmark$ & $\checkmark$ & & & $\checkmark$ & $\checkmark$ & & & $\checkmark$ & $\checkmark$ & & & $\checkmark$ & $\checkmark$ & & & $\checkmark$ & $\checkmark$ \\
\hline AC11 ... AC11 & $\checkmark$ & $\checkmark$ & $\checkmark$ & $\checkmark$ & $\checkmark$ & $\checkmark$ & & & & $\checkmark$ & & & & $\checkmark$ & & & & $\checkmark$ & & & & $\checkmark$ \\
\hline
\end{tabular}


Table 2 Descriptive Model Selection

Panel I: Trades, BA1* and AA1*

\begin{tabular}{|c|c|c|c|c|c|c|c|c|c|c|c|c|c|c|c|c|c|c|}
\hline & \multicolumn{6}{|c|}{ BMW } & \multicolumn{6}{|c|}{ SAP } & \multicolumn{6}{|c|}{ ADS } \\
\hline & Buy* & \begin{tabular}{|l|l|} 
Sell* \\
\end{tabular} & Buy & Sell & BA1* & AA1* & Buy* & Sell* & Buy & Sell & BA1* & AA1* & Buy* & Sell* & Buy & Sell & BA1* & AA1* \\
\hline Buy* & 6 & 0 & 11 & 11 & 61 & 55 & 2 & 1 & 2 & 19 & 61 & 54 & 4 & 1 & 7 & 17 & 61 & 50 \\
\hline Sell* & 1 & 2 & 8 & 7 & 52 & 61 & 1 & 1 & 14 & 4 & 57 & 61 & 1 & 1 & 10 & 8 & 53 & 58 \\
\hline Buy & 59 & 0 & 56 & 2 & 51 & 0 & 61 & 0 & 60 & 3 & 59 & 0 & 56 & 0 & 52 & 1 & 48 & 1 \\
\hline Sell & 0 & 59 & 2 & 57 & 0 & 57 & 0 & 61 & 1 & 59 & 0 & 58 & 0 & 52 & 1 & 47 & 1 & 51 \\
\hline BA1* & 45 & 61 & 4 & 44 & 49 & 5 & 25 & 61 & 5 & 45 & 21 & 3 & 50 & 60 & 8 & 26 & 55 & 5 \\
\hline AA1* & 61 & 36 & 49 & 5 & 2 & 50 & 59 & 17 & 54 & 13 & 4 & 17 & 57 & 51 & 30 & 11 & 4 & 56 \\
\hline BA1 & 1 & 0 & 0 & 0 & 59 & 0 & 1 & 0 & 0 & 0 & 61 & 0 & 3 & 0 & 0 & 0 & 58 & 0 \\
\hline BA2 & 0 & 0 & 0 & 0 & 7 & 0 & 0 & 0 & 0 & 0 & 1 & 0 & 1 & 0 & 0 & 0 & 27 & 0 \\
\hline BA3 & 0 & 0 & 0 & 0 & 0 & 0 & 0 & 0 & 0 & 0 & 0 & 0 & 0 & 0 & 0 & 0 & 3 & 0 \\
\hline BA4 & 0 & 0 & 0 & 0 & 0 & 0 & 0 & 0 & 0 & 0 & 0 & 0 & 0 & 0 & 0 & 0 & 0 & 0 \\
\hline BA5 & 0 & 0 & 0 & 0 & 0 & 0 & 0 & 0 & 0 & 0 & 0 & 0 & 0 & 0 & 0 & 0 & 0 & 0 \\
\hline BA6 & 0 & 0 & 0 & 0 & 0 & 0 & 0 & 0 & 0 & 0 & 0 & 0 & 0 & 0 & 0 & 0 & 0 & 0 \\
\hline BA7 & 0 & 0 & 0 & 0 & 0 & 0 & 0 & 0 & 0 & 0 & 0 & 0 & 0 & 0 & 0 & 0 & 0 & 0 \\
\hline BA8 & 0 & 0 & 0 & 0 & 0 & 0 & 0 & 0 & 0 & 0 & 0 & 0 & 0 & 0 & 0 & 0 & 0 & 0 \\
\hline BA9 & 0 & 0 & 0 & 0 & 1 & 0 & 0 & 0 & 0 & 0 & 0 & 0 & 0 & 0 & 0 & 0 & 0 & 0 \\
\hline BA10 & 0 & 0 & 0 & 0 & 0 & 0 & 0 & 0 & 0 & 0 & 3 & 0 & 0 & 0 & 0 & 0 & 0 & 0 \\
\hline BA11 & 0 & 0 & 0 & 0 & 0 & 1 & 0 & 0 & 0 & 0 & 0 & 0 & 0 & 5 & 0 & 0 & 0 & 0 \\
\hline BA12 & 0 & 0 & 0 & 0 & 0 & 0 & 0 & 0 & 0 & 0 & 0 & 0 & 0 & 0 & 0 & 0 & 0 & 0 \\
\hline BA13 & 0 & 0 & 0 & 0 & 0 & 0 & 0 & 0 & 0 & 0 & 1 & 0 & 0 & 0 & 0 & 0 & 0 & 0 \\
\hline BA14 & 0 & 0 & 0 & 0 & 0 & 0 & 0 & 0 & 0 & 0 & 0 & 0 & 0 & 0 & 0 & 0 & 1 & 0 \\
\hline BA15 & 0 & 0 & 0 & 0 & 1 & 0 & 0 & 0 & 0 & 0 & 0 & 1 & 0 & 0 & 0 & 0 & 0 & 0 \\
\hline BA16 & 0 & 0 & 0 & 0 & 0 & 0 & 0 & 0 & 0 & 1 & 0 & 0 & 0 & 0 & 0 & 0 & 0 & 0 \\
\hline BA17 & 0 & 0 & 0 & 0 & 0 & 0 & 0 & 0 & 0 & 0 & 0 & 0 & 0 & 0 & 0 & 0 & 0 & 0 \\
\hline BA18 & 0 & 0 & 0 & 0 & 0 & 0 & 0 & 0 & 0 & 0 & 0 & 0 & 0 & 0 & 0 & 0 & 0 & 0 \\
\hline BA19 & 0 & 0 & 0 & 0 & 0 & 0 & 0 & 1 & 0 & 0 & 0 & 0 & 0 & 0 & 0 & 0 & 0 & 0 \\
\hline BA20 & 0 & 0 & 0 & 0 & 0 & 0 & 0 & 0 & 0 & 0 & 0 & 0 & 0 & 0 & 1 & 0 & 0 & 0 \\
\hline AA1 & 0 & 2 & 0 & 0 & 0 & 61 & 0 & 3 & 0 & 0 & 0 & 60 & 0 & 8 & 0 & 0 & 0 & 58 \\
\hline AA2 & 0 & 1 & 0 & 0 & 0 & 8 & 0 & 0 & 0 & 0 & 0 & 6 & 0 & 0 & 0 & 0 & 0 & 20 \\
\hline AA3 & 0 & 0 & 0 & 0 & 0 & 1 & 0 & 0 & 0 & 0 & 0 & 0 & 0 & 0 & 0 & 0 & 0 & 4 \\
\hline AA4 & 0 & 0 & 0 & 0 & 0 & 0 & 0 & 0 & 0 & 0 & 0 & 0 & 0 & 0 & 0 & 0 & 0 & 1 \\
\hline AA5 & 0 & 0 & 0 & 0 & 0 & 0 & 0 & 0 & 0 & 0 & 0 & 0 & 0 & 0 & 0 & 0 & 0 & 0 \\
\hline AA6 & 0 & 0 & 0 & 0 & 0 & 0 & 0 & 0 & 0 & 0 & 0 & 0 & 0 & 0 & 0 & 0 & 0 & 0 \\
\hline AA7 & 0 & 0 & 0 & 0 & 0 & 0 & 1 & 0 & 0 & 0 & 0 & 0 & 0 & 0 & 0 & 0 & 0 & 0 \\
\hline AA8 & 0 & 0 & 0 & 0 & 0 & 0 & 1 & 0 & 0 & 0 & 0 & 0 & 0 & 0 & 0 & 0 & 0 & 0 \\
\hline AA9 & 0 & 0 & 0 & 0 & 0 & 0 & 0 & 0 & 0 & 0 & 0 & 0 & 0 & 0 & 0 & 0 & 0 & 0 \\
\hline AA10 & 0 & 0 & 0 & 0 & 0 & 0 & 0 & 0 & 0 & 0 & 1 & 0 & 0 & 0 & 0 & 0 & 0 & 0 \\
\hline AA11 & 3 & 0 & 0 & 0 & 2 & 0 & 0 & 0 & 0 & 0 & 0 & 0 & 0 & 0 & 0 & 0 & 0 & 0 \\
\hline AA12 & 0 & 0 & 0 & 0 & 0 & 0 & 0 & 0 & 0 & 0 & 0 & 0 & 1 & 0 & 0 & 0 & 0 & 0 \\
\hline AA13 & 0 & 0 & 0 & 0 & 0 & 0 & 0 & 0 & 0 & 0 & 0 & 0 & 0 & 0 & 0 & 0 & 0 & 0 \\
\hline AA14 & 0 & 0 & 0 & 0 & 0 & 0 & 0 & 1 & 1 & 0 & 0 & 0 & 0 & 0 & 0 & 0 & 0 & 0 \\
\hline AA15 & 0 & 0 & 0 & 0 & 0 & 1 & 0 & 0 & 0 & 0 & 0 & 0 & 0 & 0 & 0 & 0 & 0 & 0 \\
\hline AA16 & 0 & 0 & 0 & 0 & 0 & 0 & 0 & 0 & 0 & 0 & 0 & 0 & 0 & 0 & 0 & 0 & 0 & 1 \\
\hline AA17 & 0 & 0 & 0 & 0 & 0 & 0 & 0 & 0 & 0 & 0 & 0 & 0 & 1 & 0 & 0 & 0 & 0 & 0 \\
\hline AA18 & 0 & 0 & 0 & 0 & 0 & 0 & 0 & 0 & 0 & 0 & 0 & 0 & 0 & 0 & 0 & 0 & 0 & 0 \\
\hline AA19 & 0 & 0 & 0 & 0 & 0 & 0 & 0 & 0 & 0 & 0 & 0 & 0 & 0 & 0 & 0 & 0 & 0 & 0 \\
\hline AA20 & 0 & 0 & 0 & 0 & 0 & 0 & 0 & 0 & 0 & 0 & 0 & 0 & 0 & 0 & 0 & 0 & 0 & 0 \\
\hline
\end{tabular}

This table presents the number of trading days for which a given events relationship meets our selection criteria. Each column represents an explained event and each row, an explanatory event. The tone scale relates to the following categories: white: 0 to 6 days; pale grey: 7 to 30 days; dark grey: 31 to 54 days; black: 55 to 61 days. 
Panel I: Trades, BA1* and AA1* (cont.)

\begin{tabular}{|c|c|c|c|c|c|c|c|c|c|c|c|c|c|c|c|c|c|c|}
\hline & \multicolumn{6}{|c|}{ BMW } & \multicolumn{6}{|c|}{ SAP } & \multicolumn{6}{|c|}{ ADS } \\
\hline & Buy* & Sell* & Buy & Sell & BA1* & AA1* & Buy* & Sell* & Buy & Sell & BA1* & AA1* & Buy* & Sell* & Buy & Sell & BA1* & AA1* \\
\hline $\mathrm{BC} 1$ & 0 & 0 & 0 & 0 & 34 & 61 & 0 & 0 & 2 & 0 & 36 & 56 & 1 & 0 & 1 & 0 & 54 & 61 \\
\hline $\mathrm{BC} 2$ & 0 & 0 & 0 & 0 & 1 & 6 & 0 & 2 & 0 & 0 & 0 & 2 & 0 & 1 & 0 & 0 & 5 & 9 \\
\hline $\mathrm{BC} 3$ & 0 & 10 & 0 & 0 & 0 & 4 & 0 & 6 & 0 & 0 & 0 & 1 & 1 & 13 & 0 & 0 & 0 & 7 \\
\hline $\mathrm{BC} 4$ & 0 & 15 & 0 & 0 & 0 & 1 & 0 & 0 & 0 & 1 & 0 & 0 & 1 & 4 & 0 & 0 & 0 & 1 \\
\hline $\mathrm{BC} 5$ & 0 & 6 & 0 & 1 & 0 & 1 & 0 & 0 & 0 & 0 & 0 & 0 & 0 & 5 & 0 & 0 & 0 & 5 \\
\hline BC6 & 0 & 1 & 0 & 1 & 0 & 0 & 0 & 3 & 0 & 1 & 0 & 0 & 1 & 5 & 0 & 2 & 0 & 1 \\
\hline $\mathrm{BC7}$ & 0 & 1 & 0 & 0 & 0 & 0 & 0 & 2 & 0 & 0 & 0 & 0 & 0 & 8 & 0 & 0 & 0 & 0 \\
\hline $\mathrm{BC} 8$ & 0 & 0 & 0 & 0 & 0 & 0 & 0 & 0 & 0 & 1 & 0 & 0 & 0 & 4 & 0 & 1 & 0 & 1 \\
\hline BC9 & 0 & 0 & 0 & 0 & 0 & 0 & 0 & 0 & 0 & 0 & 0 & 0 & 0 & 1 & 1 & 0 & 0 & 0 \\
\hline $\mathrm{BC} 10$ & 0 & 1 & 0 & 0 & 1 & 0 & 0 & 0 & 0 & 0 & 1 & 0 & 0 & 0 & 0 & 2 & 0 & 0 \\
\hline $\mathrm{BC} 11$ & 2 & 2 & 1 & 3 & 0 & 0 & 1 & 5 & 1 & 8 & 0 & 1 & 2 & 2 & 4 & 2 & 1 & 0 \\
\hline $\mathrm{BC} 12$ & 2 & 0 & 0 & 0 & 0 & 0 & 1 & 0 & 0 & 1 & 0 & 0 & 0 & 1 & 1 & 0 & 0 & 0 \\
\hline $\mathrm{BC} 13$ & 2 & 0 & 0 & 0 & 2 & 0 & 0 & 1 & 0 & 0 & 0 & 0 & 1 & 0 & 0 & 0 & 0 & 0 \\
\hline $\mathrm{BC} 14$ & 0 & 0 & 0 & 0 & 0 & 1 & 0 & 0 & 0 & 0 & 0 & 0 & 0 & 0 & 0 & 0 & 0 & 0 \\
\hline $\mathrm{BC} 15$ & 2 & 1 & 0 & 0 & 3 & 0 & 0 & 0 & 0 & 0 & 1 & 0 & 0 & 0 & 0 & 0 & 1 & 0 \\
\hline $\mathrm{BC} 16$ & 1 & 0 & 1 & 0 & 4 & 0 & 1 & 0 & 1 & 0 & 0 & 1 & 1 & 0 & 0 & 1 & 1 & 1 \\
\hline $\mathrm{BC} 17$ & 0 & 1 & 0 & 1 & 2 & 1 & 1 & 0 & 0 & 0 & 0 & 0 & 2 & 0 & 0 & 0 & 0 & 1 \\
\hline $\mathrm{BC} 18$ & 1 & 0 & 0 & 0 & 2 & 0 & 0 & 0 & 1 & 0 & 1 & 0 & 0 & 0 & 0 & 0 & 1 & 0 \\
\hline $\mathrm{BC} 19$ & 0 & 1 & 0 & 0 & 2 & 0 & 0 & 0 & 0 & 0 & 0 & 0 & 2 & 0 & 0 & 1 & 0 & 0 \\
\hline $\mathrm{BC} 20$ & 0 & 0 & 0 & 0 & 1 & 0 & 1 & 2 & 0 & 0 & 0 & 0 & 0 & 0 & 0 & 0 & 3 & 1 \\
\hline $\mathrm{ACl}$ & 0 & 0 & 0 & 0 & 60 & 28 & 0 & 0 & 0 & 0 & 58 & 34 & 0 & 0 & 0 & 0 & 61 & 56 \\
\hline$A C 2$ & 0 & 0 & 0 & 0 & 6 & 0 & 2 & 0 & 0 & 0 & 5 & 0 & 0 & 0 & 0 & 0 & 12 & 1 \\
\hline $\mathrm{AC} 3$ & 9 & 0 & 1 & 0 & 11 & 0 & 1 & 0 & 0 & 0 & 0 & 0 & 8 & 0 & 0 & 0 & 8 & 1 \\
\hline $\mathrm{AC} 4$ & 10 & 0 & 0 & 0 & 1 & 0 & 0 & 0 & 0 & 0 & 0 & 0 & 7 & 0 & 0 & 0 & 4 & 0 \\
\hline AC5 & 8 & 0 & 0 & 0 & 0 & 0 & 0 & 0 & 0 & 0 & 0 & 0 & 4 & 0 & 0 & 0 & 4 & 1 \\
\hline AC6 & 1 & 0 & 0 & 0 & 0 & 0 & 1 & 0 & 0 & 0 & 0 & 0 & 12 & 0 & 0 & 0 & 0 & 0 \\
\hline $\mathrm{AC7}$ & 3 & 0 & 1 & 0 & 0 & 0 & 4 & 0 & 1 & 0 & 1 & 0 & 6 & 0 & 0 & 0 & 2 & 1 \\
\hline AC8 & 1 & 0 & 0 & 0 & 0 & 0 & 0 & 0 & 0 & 0 & 0 & 0 & 3 & 0 & 0 & 0 & 0 & 0 \\
\hline AC9 & 1 & 0 & 0 & 0 & 0 & 0 & 0 & 0 & 0 & 0 & 0 & 0 & 1 & 0 & 1 & 0 & 0 & 0 \\
\hline AC10 & 0 & 0 & 0 & 0 & 0 & 0 & 0 & 0 & 0 & 0 & 0 & 0 & 0 & 0 & 2 & 0 & 0 & 0 \\
\hline $\mathrm{AC} 11$ & 1 & 2 & 3 & 1 & 1 & 1 & 2 & 2 & 7 & 1 & 2 & 0 & 4 & 2 & 6 & 1 & 0 & 0 \\
\hline $\mathrm{AC} 12$ & 0 & 1 & 0 & 0 & 1 & 0 & 0 & 0 & 0 & 1 & 0 & 2 & 0 & 1 & 0 & 0 & 0 & 2 \\
\hline $\mathrm{AC} 13$ & 0 & 2 & 0 & 0 & 0 & 0 & 0 & 0 & 0 & 0 & 0 & 1 & 0 & 0 & 1 & 0 & 0 & 0 \\
\hline AC14 & 0 & 3 & 0 & 0 & 1 & 0 & 0 & 0 & 0 & 0 & 0 & 0 & 0 & 0 & 0 & 0 & 1 & 1 \\
\hline $\mathrm{AC} 15$ & 0 & 1 & 0 & 0 & 0 & 3 & 0 & 0 & 0 & 0 & 0 & 0 & 0 & 0 & 0 & 0 & 0 & 1 \\
\hline $\mathrm{AC} 16$ & 0 & 5 & 0 & 0 & 0 & 3 & 0 & 0 & 0 & 0 & 0 & 0 & 0 & 1 & 0 & 0 & 1 & 1 \\
\hline $\mathrm{AC} 17$ & 0 & 0 & 0 & 1 & 0 & 2 & 0 & 0 & 0 & 0 & 0 & 1 & 0 & 0 & 0 & 0 & 0 & 0 \\
\hline $\mathrm{AC} 18$ & 2 & 1 & 1 & 0 & 0 & 1 & 0 & 2 & 0 & 0 & 1 & 0 & 0 & 0 & 0 & 1 & 0 & 2 \\
\hline AC19 & 1 & 1 & 1 & 0 & 1 & 1 & 0 & 3 & 0 & 0 & 0 & 0 & 1 & 0 & 0 & 0 & 0 & 1 \\
\hline$A C 20$ & 0 & 1 & 0 & 0 & 0 & 1 & 0 & 0 & 0 & 0 & 0 & 0 & 0 & 0 & 0 & 0 & 1 & 0 \\
\hline
\end{tabular}




\section{Panel II: BA1 to BA10}

\begin{tabular}{|c|c|c|c|c|c|c|c|c|c|c|c|c|c|c|c|c|c|c|c|c|c|c|c|c|c|c|c|c|c|c|}
\hline & \multicolumn{10}{|c|}{ BMW } & \multicolumn{10}{|c|}{ SAP } & \multicolumn{10}{|c|}{ ADS } \\
\hline $\mathrm{BA}$ & 1 & 2 & 3 & 4 & 5 & 6 & 7 & 8 & $y$ & 10 & 1 & 2 & 3 & 4 & 5 & & & 8 & 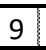 & 10 & 1 & 2 & 3 & & & & 7 & 8 & & \\
\hline $\mathrm{y}^{*}$ & 61 & 61 & 60 & 59 & 48 & 26 & 22 & 10 & 1 & 0 & 60 & 61 & 52 & 44 & 47 & 25 & 16 & 14 & 1 & 1 & 60 & 61 & 616 & 60 & 47 & 28 & 17 & 21 & 2 & \\
\hline I* & $\begin{array}{ll}61 \\
\end{array}$ & 39 & 61 & 54 & 44 & 34 & 30 & 42 & 60 & 61 & 61 & 33 & 55 & 40 & 39 & 46 & 50 & 45 & 54 & 60 & 61 & 31 : & $55 \quad 5$ & 53 & & 23 & & 44 & 61 & \\
\hline dy & 55 & 59 & 58 & 27 & 7 & 2 & \begin{tabular}{|l|}
1 \\
\end{tabular} & 2 & 0 & 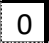 & 55 & 48 & 32 & & 3 & & 0 & 0 & 0 & 0 & $57:$ & 55 & 441 & 17 & 8 & 5 & 4 & 3 & 0 & \\
\hline ell & 22 & 15 & 12 & 5 & 4 & 8 & 3 & 3 & 3 & 6 & \begin{tabular}{|l|}
37 \\
\end{tabular} & 17 & 8 & 3 & 2 & & & 1 & 0 & 12 & 25 & 4 & 7 & 4 & & 2 & 0 & 2 & 0 & \\
\hline$*$ & 61 & 61 & 61 & 60 & 53 & 59 & 28 & 4 & 0 & 1 & 59 & 59 & 60 & 58 & 48 & 42 & 32 & 4 & 1 & 12 & 59 & 61 & 616 & 61 & 49 & 51 & 44 & 9 & 1 & \\
\hline $\mathrm{A} 1 *$ & 2 & 23 & 47 & 14 & 3 & 23 & 22 & 25 & 13 & 17 & 13 & 23 & 23 & 8 & 9 & 11 & 11 & 5 & 43 & 19 & 7 & 19 & \begin{tabular}{l|l}
88 & 1
\end{tabular} & 12 & 8 & 12 & 23 & 21 & 24 & \\
\hline $\mathrm{A1}$ & 1 & 61 & 61 & 57 & 14 & 0 & $\begin{array}{l}11 \\
1\end{array}$ & 0 & 0 & 0 & 61 & 60 & 59 & 13 & 8 & & & 0 & 0 & 2 & 56 & 61 & 614 & 47 & 23 & 2 & 1 & \begin{tabular}{|l|}
1 \\
\end{tabular} & 0 & \\
\hline 12 & 7 & 59 & 59 & 21 & 5 & 0 & 1 & 0 & 0 & 0 & 39 & 19 & 50 & 13 & 4 & & & 0 & 0 & 0 & 29 & 54 & $\begin{array}{lll}55 & 1\end{array}$ & 11 & 2 & 3 & 2 & 0 & 0 & \\
\hline 13 & 26 & 59 & 60 & 35 & 6 & 1 & 1 & 0 & 0 & 0 & 4 & 51 & 40 & 41 & 17 & 0 & & 0 & 0 & 0 & & 43 & 554 & 43 & 23 & 16 & 10 & 1 & 0 & \\
\hline 44 & & & & 25 & 51 & 3 & 3 & 0 & 0 & 0 & & & & 7 & 38 & 0 & $c$ & 0 & 0 & 0 & & & & 7 & 32 & 9 & 4 & 0 & 0 & \\
\hline A5 & & & & 49 & 17 & 47 & 6 & 1 & 2 & 3 & & & & 3 & 0 & 29 & 0 & 0 & 0 & 0 & & & & 29 & 8 & 43 & 4 & & 0 & \\
\hline A6 & & & & 13 & 24 & 2 & 42 & 10 & 1 & 14 & & & & 3 & 3 & 1 & 32 & 3 & 0 & 2 & & & & & 38 & 1 & 45 & 5 & 0 & \\
\hline A7 & & & & & & & 0 & 52 & 7 & 9 & & & & & & & 0 & 43 & 2 & 5 & & & & & & & & 53 & 4 & \\
\hline$A 8$ & & & & & & & 9 & 1 & 58 & 7 & & & & & & & 7 & 0 & 39 & 5 & & & & & & & 6 & & 55 & \\
\hline A9 & & & & & & & 10 & 32 & 1 & 59 & & & & & & & 1 & 12 & 0 & 53 & & & & & & & & 28 & 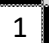 & \\
\hline 3A10 & & & & & & & 9 & 4 & 0 & 0 & & & & & & & & & 0 & 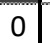 & & & & & & & & 2 & 0 & \\
\hline A1 & 1 & 0 & 0 & 0 & 0 & 0 & 0 & 0 & - & 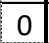 & 1 & 0 & 1 & 1 & 0 & & & & 0 & 0 & 0 & 0 & 0 & 0 & & 0 & 0 & & 0 & \\
\hline A2 & 0 & 1 & 7 & 2 & 1 & 1 & 1 & 0 & 0 & 7 & 0 & 0 & & 1 & 2 & & & & 0 & 0 & 0 & 0 & 0 & & 0 & & & & 0 & \\
\hline A3 & 0 & 0 & 49 & 11 & 0 & 0 & 0 & 0 & 0 & & 0 & 1 & 22 & $\epsilon$ & 3 & & & & 0 & 0 & 0 & 0 & 0 & & $c_{-}$ & 3 & & & 0 & \\
\hline A4 & & & & 27 & 0 & 0 & 0 & 0 & 0 & & & & & 7 & 0 & & & 0 & 0 & & & & & 18 & $c$ & 0 & & & 0 & \\
\hline A5 & & & & 8 & 8 & 2 & 1 & 0 & 0 & & & & & C & 3 & & & 0 & 0 & & & & & 1 & 4 & 0 & & & 0 & \\
\hline AA6 & & & & 4 & 2 & 2 & 0 & 0 & 0 & & & & & 4 & 4 & & & & 0 & & & & & & 0 & 0 & & & 0 & \\
\hline A77 & & & & & & & 0 & 0 & 0 & & & & & & & & & & 0 & & & & & & & & & 0 & 0 & \\
\hline AA8 & & & & & & & 1 & 2 & 0 & & & & & & & & & & 0 & & & & & & & & 0 & 0 & 0 & \\
\hline $\mathrm{AA9}$ & & & & & & & 2 & 0 & 0 & 7 & & & & & & & & & 0 & & & & & & & & & 0 & 0 & 7 \\
\hline A10 & & & & & & & 0 & 0 & 0 & & & & & & & & $c$ & & 1 & 9 & & & & & & & & 0 & 0 & \\
\hline 61 & & 55 & 61 & 29 & 24 & 18 & 1 & 23 & 2 & 59 & 61 & 48 & 60 & 31 & 8 & 35 & 13 & 19 & 0 & 56 & 61 & 61 & 53 & 32 & 26 & 10 & & & 19 & \\
\hline$C 2$ & 34 & 42 & 60 & 37 & 1 & 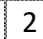 & & 14 & 24 & 0 & 39 & 45 & 56 & 49 & 13 & 2 & 13 & 3 & 52 & 0 & 48 & 55 & 60 & 42 & 5 & 5 & & & 58 & \\
\hline $\mathrm{BC} 3$ & 21 & 59 & 60 & 59 & 23 & 4 & 11 & 10 & 0 & 59 & 12 & 38 & 61 & 56 & 39 & 1 & 2 & 1 & 0 & 2 & & 54 & 30 & 61 & 20 & 5 & 7 & 19 & 0 & $\epsilon$ \\
\hline $\mathrm{BC4}$ & & & & 46 & 51 & 17 & \begin{tabular}{|l|}
5 \\
\end{tabular} & 5 & 0 & 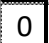 & & & & 61 & 36 & 36 & 0 & 0 & 0 & c & & & & 28 & 50 & 14 & 4 & 4 & 0 & \\
\hline $3 C 5$ & & & & 50 & 57 & 19 & 4 & 2 & 8 & $c$ & & & & 29 & 59 & 23 & 8 & 0 & 1 & & & & & 60 & 57 & 33 & 3 & 2 & 10 & \\
\hline$C E$ & & & & 55 & 11 & 57 & 0 & 4 & 0 & 1 & & & & 43 & 14 & 61 & 11 & 0 & 0 & c & & & & 59 & & 59 & 9 & \begin{tabular}{|l|} 
\\
\end{tabular} & 0 & 8 \\
\hline$C 7$ & & & & & & & 42 & 6 & 0 & 0 & & & & & & & 43 & 5 & 0 & $c$ & & & & & & & 43 & 15 & 4 & \\
\hline 18 & & & & & & & 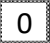 & 43 & 14 & D & & & & & & & & 37 & 1 & & & & & & & & 7 & 39 & 16 & \\
\hline 30 & & & & & & & 2 & 4 & 19 & & & & & & & & & 1 & 8 & 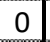 & & & & & & & 5 & 3 & 18 & 5 \\
\hline $3 \mathrm{C} 10$ & & & & & & & 4 & 0 & 1 & 1. & & & & & & & & 0 & 2 & 4 & & & & & & & 3 & 2 & 8 & \\
\hline C1 & & 61 & 6 & 59 & 28 & & 0 & 0 & 0 & 0 & 61 & 58 & 58 & 41 & 3 & & & 0 & 0 & 0 & 61 & 61 & & 61 & 53 & 4 & 0 & 0 & 0 & \\
\hline C? & 54 & 57 & 59 & 22 & 1 & & 0 & 0 & 0 & 0 & 44 & 51 & 52 & 7 & 0 & & & 0 & 0 & 0 & 33 & 56 & 56 & 3 & 3 & 1 & 0 & 0 & 0 & \\
\hline$A C 3$ & 57 & 60 & 61 & 46 & 3 & & 1 & 0 & 0 & 0 & 29 & 33 & 54 & & 1 & & & 0 & 0 & 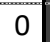 & 37 & & 59 & 3 & 9 & 0 & 0 & 0 & 0 & \\
\hline ro & & & & 26 & 3 & & 1 & 0 & 0 & 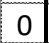 & & & & & 2 & & & 0 & $c$ & 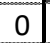 & & & & 31 & 6 & 6 & 2 & 0 & 0 & $c$ \\
\hline & & & & 8 & 0 & & 0 & 0 & 0 & 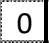 & & & & & 8 & & & 0 & $c$ & 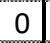 & & & & 10 & 6 & 5 & 5 & 1 & 0 & 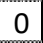 \\
\hline re & & & & 19 & 0 & 0 & 0 & 1 & 0 & 8 & & & & 5 & 3 & & $t_{-1}$ & 0 & 0 & 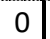 & & & & 20 & 0 & 0 & 3 & 1 & 0 & 8 \\
\hline $1 C^{-1}$ & & & & & & & 1 & 7 & 2 & $c$ & & & & & & & 1 & 5 & 0 & 0 & & & & & & & 0 & 1 & 4 & 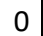 \\
\hline $\mathrm{AC} 8$ & & & & & & & 0 & 1 & 1 & 2 & & & & & & & 1 & 0 & 0 & $c$ & & & & & & & 1 & 0 & 0 & 3 \\
\hline CC9 & & & & & & & 1 & 1 & 0 & 0 & & & & & & & $c$ & 1 & 0 & 2 & & & & & & & & 3 & 0 & 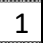 \\
\hline C10 & & & & & & & 6 & & 1 & & & & & & & & & 7 & 3 & 3 & & & & & & & & & 1 & \\
\hline
\end{tabular}

Notes: Each column represents the depth level number on which the explained Bid order Added event occurs. Hatched cells correspond to relationship subject to the model initial restrictions. The same applies to absent explanatory events. 
Panel III: BA11 to BA20

\begin{tabular}{|c|c|c|c|c|c|c|c|c|c|c|c|c|c|c|c|c|c|c|c|c|c|c|c|c|c|c|c|c|c|c|}
\hline & \multicolumn{10}{|c|}{ BMW } & \multicolumn{10}{|c|}{ SAP } & \multicolumn{10}{|c|}{ DS } \\
\hline$B A$ & & 12 & 13 & 1 & 15 & & & & & & & & & 1 & 15 & & 7) & & & & & & 13 & 14 & & & & & & \\
\hline$v^{*}$ & 0 & 0 & 2 & $\overline{6}$ & 0 & & & 0 & & & & 2 & 25 & 12 & 0 & & & 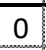 & 0 & 0 & \begin{tabular}{l|l}
0 \\
\end{tabular} & 0 & 0 & 0 & & \begin{tabular}{l|l}
3 & \\
\end{tabular} & & 3 & 2 & \\
\hline & & 27 & 34 & 40 & 46 & & & 4 & & & & 36 & 44 & 51 & 45 & $3=$ & 7 & 0 & & & 47 & 31 & 37 & 32 & 43 & 44 & & & & \\
\hline & 0 & 0 & $\pi$ & 0 & 0 & & & 0 & & 0 & & 0 & 0 & 0 & 0 & 0 & 0 & 0 & 0 & 0 & & & & 0 & & & 0 & 0 & 0 & \\
\hline & 46 & 1 & & 0 & & 0 & & & & & & 1 & & c & 0 & & & & & 0 & & & & & & O & & & 0 & \\
\hline $1 *$ & 0 & 2 & & 3 & 14 & & 17 & & & & & & & 17. & 11 & 0 & 0 & & & 0 & & 0 & 10 & 6 & & 13 & & 20 & 0 & \\
\hline $\mathrm{A1}^{*}$ & 11 & & & 7 & & & & & & & & 2 & $?$ & 12 & 16 & 14 & 0 & O & 0 & & & 13 & 11 & 10 & & & & 0 & 0 & \\
\hline $\mathrm{C} 1$ & 0 & 0 & 0 & 0 & 0 & 0 & & & & & & & 0 & & & & 0 & & & & & & & 1 & 0 & & & & & \\
\hline C2 & 1 & 0 & & 0 & 20 & 0 & c & & & & & & 0 & $c$ & & & 0 & 0 & 0 & & & & & 0 & 18 & 0 & 0 & 0 & & \\
\hline 3 & 0 & 3 & 0 & 0 & 0 & 31 & 0 & 0 & & & $c$ & & 0 & c & 0 & 1 & 0 & 0 & 0 & 0 & 0 & & 0 & 0 & 1 & 27 & 0 & 0 & 0 & \\
\hline 4 & 56 & 8 & 0 & 0 & 0 & 0 & 5 & 0 & & & & & 0 & c & 0 & 0 & 0 & 0 & 0 & 0 & 51 & 0 & 0 & 0 & & & 5 & 0 & 0 & \\
\hline C5 & 0 & 14 & 0 & & & & & & & & & & 0 & & & & & & & 0 & 0 & 17 & 0 & & & & & 0 & & \\
\hline C6 & 0 & & & 2 & & & & & & & & & & & & & & & & & & & 6 & & & & & & & \\
\hline C7 & & & & 4 & & & & & & & & & & & & & & & & & & & & & & & & & & \\
\hline C8 & 0 & & & 0 & & & & & & & & & & & & & & & & & & & & & & & & & U & \\
\hline 9 & 3 & & & 1 & & & & & & & & & & & & & & & & & & & & & & & & & 0 & \\
\hline 10 & 0 & & & 9 & & & & & & & & & & & & & & & & & & & & & & & & U & 0 & \\
\hline C11 & 1 & & & 3 & & & & & & & & & & 36 & 16 & & & & & & & & & & & 13 & 5 & 15 & 11 & \\
\hline 12 & 0 & & & 3 & & & & & & & & & 14 & 7 & & & & & & & & & & & & & & & & \\
\hline 613 & 0 & & & 4 & 14 & 14 & & & & & & & 9 & 2 & & & 0 & & & & & & & & & & & 0 & & \\
\hline C14 & 0 & & 0 & 15 & 3 & & & & & & & & 2 & & & & 0 & O & & & & & & & & & & 0 & 0 & \\
\hline 15 & ח & & & 0 & & & & & & & & & 1 & 1 & 15 & 0 & & & & & & & & & & 0 & & 0 & & \\
\hline & & & & & & & & & & & & & & & & 21 & 1 & & & & & & 0 & & & 11 & & & & \\
\hline 617 & & & 4 & 2 & & & 14 & & & & C & & 6 & & & & & & & 1 & & & 0 & & & & 11 & 0 & & \\
\hline 618 & & 1 & & 5 & & & & & & & & & & & & & & & & () & & & 6 & & & & & & & \\
\hline 19 & & & & 17 & & & & & & & & & & & & & & & & & & & 4 & 11 & & & & & & \\
\hline 220 & & & & & & & & & & & & & & & & & & & & & & & & & & & & & 0 & \\
\hline 61 & c & & & & & & & & & & & & & & & & & & & & & & & & & & & 0 & 0 & \\
\hline C2 & c & & & & & & & & & & & & & & & & & & & & & & & & & & & 0 & 0 & \\
\hline AC3 & c & & & & & & & & & & & & & & & & & & & & & & & & & & & 0 & & \\
\hline $\mathrm{AC4}$ & & & & & & & & & & & & & & & & & & & & & & & & & & & & 0 & & \\
\hline $\mathrm{AC5}$ & C & & & & & & & & & & & & & & & & & & & & & & & & & C & & 0 & 0 & \\
\hline $\mathrm{AC6}$ & C & & & & & & & & & & & & & & & & & & & & & & 0 & & & 0 & & 0 & 0 & \\
\hline AC7 & C & & & & & & & & & & & & & & & & & & & & & & 0 & & & 0 & & 0 & & \\
\hline $\mathrm{AC}$ & 0 & & & & & & & & & & & & & I & & & & & & 0 & & & 0 & & & & & U & 0 & \\
\hline & & & & & & & & & & & & & & & & & & & & & & & 1 & & & & & $\perp$ & & \\
\hline АCIC & & & & 2 & & & & & & & & & & & & & & & & U & & & 1 & & & & & $\perp$ & 0 & \\
\hline $\mathrm{C} 11$ & 1 & & & 1 & & & & & & & & & & $\varepsilon$ & & & & & & & & & 1 & & & & & & & \\
\hline $\mathrm{C} 12$ & 2 & & & $\epsilon$ & & & & & & & & & & ( & & & & & 5 & & & & 6 & 1 & & & & & 0 & \\
\hline & c & & & & & & & & & & & & & & & & & & & & & & 0 & . & & 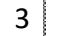 & & 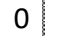 & 0 & \\
\hline C14 & 1 & & & 1 & & & & & & & & & & ( & & & & & 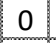 & & & & 1 & 2 & & 0 & & 0 & 0 & \\
\hline & & & & & & & & & & & & & & . & & & & & & & & & 0 & 0 & & & & 0 & 0 & \\
\hline & & 2 & & 3 & & & & & & & 2 & & & 1 & & & c & & $c$ & 0 & & & 1 & 2 & 0 & 0 & 0 & 0 & 0 & \\
\hline$C$ & & & & 3 & & & & & & & 3 & & & 1 & & & & 0 & . & 1 & $c$ & & 3 & 4 & 2 & 1 & 0 & 0 & 0 & \\
\hline & 5 & & & & & & & & & & & & & 3 & & & & & & & & & & C & & & & 0 & & \\
\hline & 2 & & 0 & c & 0 & 0 & & & & & C & 0 & & $c$ & 0 & C & & 0 & 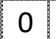 & U & 0 & & 2 & 2 & & 0 & 0 & 0 & 0 & \\
\hline & & 1 & & $c$ & & & & & & & 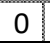 & & & 0 & & & 0 & & 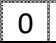 & 0 & & 0 & 1 & 1 & 0 & 0 & & & 0 & \\
\hline
\end{tabular}

Note: Due to the general absence of relationships involving these explanatory events, BA11 to BA20 and AA11 to AA20 are omitted. 


\section{Panel IV: BC1 to BC10}

\begin{tabular}{|c|c|c|c|c|c|c|c|c|c|c|c|c|c|c|c|c|c|c|c|c|c|c|c|c|c|c|c|c|c|c|}
\hline & \multicolumn{10}{|c|}{ BMW } & \multicolumn{10}{|c|}{ SAP } & \multicolumn{10}{|c|}{ ADS } \\
\hline$B C$ & 1 & 2 & 3 & 4 & 5 & 6 & 7 & 8 & 9 & 10 & 1 & 2 & 3 & 4 & 5 & 6 & 1 & 8 & 9 & 10 & & 2 & 3 & 4 & 5 & & 7 & 8 & & \\
\hline dy* & 15 & 34 & 44 & 48 & 49 & 33 & 21 & 22 & 33 & 52 & 19 & 27 & 40 & 51 & 57 & 34 & 31 & 16 & 29 & 52 & 13 & 42 & 45 & 52 & 51 & 32 & 16 & 25 & 34 & \\
\hline$\| *$ & 61 & 61 & 61 & 60 & 60 & 61 & 60 & 50 & 46 & 13 & 61 & 61 & 61 & 59 & 58 & 59 & 54 & 33 & 17 & 29 & 61 & 61 & 61 & 61 & 61 & 59 & 58 & 47 & 35 & \\
\hline dy & 12 & 19 & 21 & 15 & 13 & 5 & 2 & 1 & 0 & 1 & 4 & 11 & 7 & 21 & 0 & 4 & 3 & 2 & 0 & 0 & & 15 & 15 & 7 & 10 & 11 & 0 & 0 & 0 & \\
\hline ell & 59 & 58 & 61 & 57 & 49 & 45 & 31 & 36 & 23 & 0 & 59 & 61 & 58 & 53 & 44 & 30 & 20 & 14 & 0 & 10 & 58 & 56 & 53 & 40 & 29 & 33 & 19 & 13 & 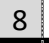 & \\
\hline A1* & 23 & 61 & 61 & 61 & 61 & 61 & 61 & 60 & 61 & 61 & 12 & 61 & 58 & 61 & 58 & 61 & 61 & 59 & 58 & 61 & 9 & 58 & 59 & 61 & 61 & 61 & 60 & 57 & 60 & \\
\hline $\mathrm{A}^{*}$ & 61 & 61 & 61 & 0 & 59 & 44 & 13 & 5 & 4 & 1 & 61 & 60 & 54 & 51 & 42 & 27 & 23 & 6 & 1 & 2 & 61 & 61 & 61 & 60 & 57 & 54 & 31 & 5 & 2 & \\
\hline 41 & 57 & 55 & 27 & 40 & 8 & 1 & 1 & 0 & 0 & 0 & 61 & 36 & 32 & 27 & 2 & 0 & 0 & 0 & 0 & 0 & 52 & 33 & 4 & 5 & 2 & 0 & 0 & 0 & 0 & \\
\hline 42 & 0 & 38 & 2 & 11 & 16 & 10 & 15 & 0 & 0 & 0 & 0 & 55 & 6 & 17 & 8 & 4 & 14 & 0 & 0 & 0 & 4 & 24 & 4 & 3 & 1 & 4 & 22 & 7 & 0 & \\
\hline 43 & 0 & 2 & 40 & 0 & 1 & 1 & 0 & 4 & 0 & 0 & 0 & 5 & 55 & 6 & 8 & & & 0 & 0 & 0 & 0 & 3 & 26 & 4 & 1 & 2 & & & 8 & \\
\hline 14 & & & & 29 & 0 & 2 & 0 & 3 & 4 & 0 & & & & 59 & 2 & 0 & (5) & 0 & 0 & 0 & & & & 10 & 2 & 1 & 0 & 0 & 12 & \\
\hline 15 & & & & 0 & 41 & 1 & 0 & 0 & 7 & 4 & & & & 1 & 59 & 0 & 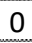 & 0 & 0 & & & & & 2 & 28 & 0 & 0 & 1 & 10 & 1 \\
\hline 16 & & & & 0 & 0 & 25 & 1 & 0 & 0 & 3 & & & & 0 & 0 & 57 & 0 & 1 & 0 & & & & & 1 & 1 & 16 & 1 & 0 & 1 & \\
\hline 47 & & & & & & & 2 & 3 & 0 & 1 & & & & & & & 2 & 8 & 1 & & & & & & & & 3 & 5 & 1 & \\
\hline $\mathrm{A} 8$ & & & & & & & 1 & 2 & 0 & 0 & & & & & & & $c$ & 4 & 4 & & & & & & & & 0 & 0 & 2 & \\
\hline A9 & & & & & & & 0 & 0 & 2 & 0 & & & & & & & 2 & 3 & 4 & & & & & & & & 0 & 0 & 4 & \\
\hline 0 & & & & & & & 2 & 0 & 0 & 1 & & & & & & & 1 & U & 0 & & & & & & & & & & 0 & \\
\hline A1 & 60 & 61 & 61 & 29 & 3 & 0 & 0 & 0 & 0 & 0 & 61 & 60 & 42 & 8 & 1 & 0 & $c$ & 0 & 0 & 0 & 61 & 60 & 59 & 25 & 10 & 4 & 1 & & 0 & \\
\hline $\mathrm{A} 2$ & 52 & 54 & 52 & 21 & 1 & 1 & 0 & 0 & 0 & 0 & 53 & 55 & 22 & 4 & 0 & U & & 0 & 0 & 0 & 51 & 49 & 49 & 22 & 7 & 2 & 0 & & 0 & \\
\hline 33 & 35 & 51 & 54 & 6 & $c$ & 0 & 0 & 0 & 0 & 0 & 27 & 49 & 20 & 2 & 1 & & & U & 0 & 0 & 40 & 52 & 52 & 19 & 4 & 3 & 0 & & 0 & \\
\hline 44 & & & & 1 & 0 & 0 & 0 & 0 & 0 & c & & & & 0 & 1 & & C & U & 0 & & & & & 6 & 0 & 1 & 1 & & 0 & \\
\hline A5 & & & & 4 & 0 & 0 & 0 & 0 & 0 & c & & & & 0 & 0 & & $C^{-1}$ & & 0 & & & & & & 2 & 0 & 0 & 0 & 0 & \\
\hline A6 & & & & 0 & 1 & & 0 & 0 & 0 & & & & & 2 & 2 & & & U & 0 & & & & & 0 & 0 & 0 & 0 & 0 & 0 & \\
\hline AA7 & & & & & & & 0 & 0 & 0 & & & & & & & & & & 0 & & & & & & & & 0 & 0 & 0 & \\
\hline AA8 & & & & & & & 0 & 0 & 1 & & & & & & & & & U & & & & & & & & & & & 0 & \\
\hline A99 & & & & & & & 1 & & 0 & & & & & & & & & & 0 & & & & & & & & 0 & 0 & 0 & \\
\hline $\mathrm{AA10}$ & & & & & & & 0 & & C & & & & & & & & & & 0 & & & & & & & & 0 & 0 & 0 & \\
\hline $3 \mathrm{C} 1$ & 61 & 61 & 61 & $\epsilon$ & 49 & 2 & 2 & 0 & 0 & 0 & & 61 & 61 & 50 & 27 & 5 & ( & 0 & 0 & 0 & 1 & 61 & 61 & 61 & 58 & 25 & 5 & 1 & 1 & \\
\hline $3 C 2$ & 61 & 61 & 61 & 3 & 17 & 0 & 0 & 0 & 0 & 0 & 61 & 61 & 60 & 43 & 14 & 1 & ( & 0 & 0 & 0 & 1 & 60 & 61 & 37 & 41 & 18 & 1 & 0 & 0 & \\
\hline C3 & 59 & 61 & 60 & 60 & 46 & 36 & 8 & 0 & 0 & 0 & 43 & 60 & 50 & 45 & 46 & 3 & 0 & 0 & 0 & 0 & 55 & 61 & 60 & 49 & 48 & 44 & 23 & 2 & 0 & \\
\hline $3 C 4$ & & & & 52 & 48 & 43 & 18 & 3 & 0 & 1 & & & & 6 & 25 & 20 & $c$ & 0 & 0 & I & & & & 35 & 46 & 46 & 31 & 8 & 1 & ( \\
\hline $3 C 5$ & & & & 47 & 19 & 43 & 15 & 14 & 0 & 0 & & & & 37 & 2 & 19 & 2 & 0 & 0 & I & & & & 52 & 22 & 50 & 34 & & 4 & 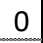 \\
\hline c6 & & & & 23 & 36 & 25 & 43 & 10 & 2 & 1 & & & & 6 & 17 & 9 & 8 & 1 & 0 & $c$ & & & & 25 & 30 & 2 & 32 & 26 & 3 & 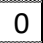 \\
\hline $3 C 7$ & & & & & & & 33 & 16 & 5 & 4 & & & & & & & 1 & 22 & 6 & 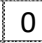 & & & & & & & 16 & 30 & 23 & 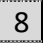 \\
\hline $3<\varepsilon$ & & & & & & & 21 & 7 & 6 & 3 & & & & & & & $\varepsilon$ & 1 & 9 & & & & & & & & 25 & 1 & 15 & $s$ \\
\hline $3 C 9$ & & & & & & & 2 & 13 & 1 & 4 & & & & & & & 5 & 14 & 3 & 5 & & & & & & & 1 & 12 & 1 & 1 \\
\hline $3 \mathrm{C} 10$ & & & & & & & 19 & 14 & 28 & 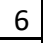 & & & & & & & & 11 & 14 & & & & & & & & 8 & 5 & 26 & 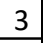 \\
\hline & 5 & 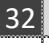 & 29 & 44 & 15 & & 1 & 0 & 1 & $\epsilon$ & 0 & 11 & 24 & 39 & 11 & 1 & & 1 & 0 & 1 & & 16 & 8 & 33 & 5 & 0 & 0 & 0 & 0 & \\
\hline & 0 & 4 & 6 & 0 & 0 & & 1 & 0 & 0 & $c$ & 0 & 3 & 11 & 7 & 5 & & & 0 & 0 & 0 & & 4 & 3 & 0 & 1 & 0 & 0 & 0 & 0 & ( \\
\hline & 1 & 20 & 39 & 1 & 6 & & 0 & 0 & 0 & c & 0 & 15 & & 16 & 3 & & & 0 & 0 & 0 & & 8 & 12 & & 5 & 4 & 2 & 0 & 0 & \\
\hline C & & & & 1 & 1 & & c & 0 & 0 & & & & & 2 & 16 & & & 0 & 0 & - & & & & $\Delta$ & 1 & 0 & 0 & 1 & 0 & \\
\hline & & & & 6 & 4 & & $c$ & 0 & 0 & 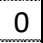 & & & & 1 & & 3 & & 0 & 0 & & & & & 5 & 3 & 1 & 0 & 0 & 0 & \\
\hline & & & & 13 & 4 & & 0 & 0 & 0 & 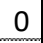 & & & & 5 & 10 & & & 0 & 0 & 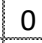 & & & & 4 & 2 & 1 & 0 & 0 & 0 & $c$ \\
\hline & & & & & & & 0 & 1 & 0 & c & & & & & & & C & 0 & 0 & C & & & & & & & 1 & 0 & 0 & (1) \\
\hline & & & & & & & 0 & 0 & 0 & ( & & & & & & & $y$ & 1 & 1 & & & & & & & & 1 & 0 & 0 & 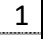 \\
\hline & & & & & & & 0 & 0 & 0 & ( & & & & & & & 1 & 1 & 1 & & & & & & & & 0 & 0 & 1 & 4 \\
\hline C10 & & & & & & & & 6 & & & & & & & & & & & & & & & & & & & & & & \\
\hline
\end{tabular}




\section{Panel V: BC11 to BC20}

\begin{tabular}{|c|c|c|c|c|c|c|c|c|c|c|c|c|c|c|c|c|c|c|c|c|c|c|c|c|c|c|c|c|c|c|}
\hline & \multicolumn{10}{|c|}{ BMW } & \multicolumn{10}{|c|}{ SAP } & \multicolumn{10}{|c|}{ ADS } \\
\hline$B C$ & \begin{tabular}{|c|}
11 \\
\end{tabular} & & \begin{tabular}{|l|}
13 \\
\end{tabular} & \begin{tabular}{|l|}
14 \\
\end{tabular} & & & \begin{tabular}{|c|}
$17 \mid$ \\
\end{tabular} & 18 & \begin{tabular}{|l|}
19 \\
\end{tabular} & & 11 & & & 14 & & & \begin{tabular}{|c|}
17 \\
\end{tabular} & & 19 & & & & \begin{tabular}{|l|}
13 \\
\end{tabular} & \begin{tabular}{|l|}
14 \\
\end{tabular} & & & & & & \\
\hline Buy* & 54 & 51 & 44 & 36 & 41 & 40 & 29 & 30 & $\overline{15}$ & 1 & 42 & 39 & 35 & 43 & 37 & 49 & 38 & \begin{tabular}{|l|}
23 \\
\end{tabular} & o & 0 & & 50 & 38 & 28 & 42 & 44 & 34 & 311 & $\begin{array}{ll}12 & \end{array}$ & \\
\hline Sell* & 7 & 5 & 7 & 6 & 1 & 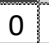 & 0 & 0 & 0 & 0 & 15 & 3 & - & 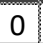 & 0 & 0 & 0 & 0 & 0 & 0 & 5 & 10 & 7 & 9 & 8 & 0 & \begin{tabular}{l|l} 
\\
\end{tabular} & 1 & 0 & \\
\hline Buy & 3 & 1 & 2 & 0 & 1 & 0 & 0 & 0 & 0 & 0 & & 1 & 0 & 0 & 0 & 0 & 0 & 0 & $\mathrm{c}$ & 0 & 0 & \begin{tabular}{|l|}
2 \\
\end{tabular} & 0 & 0 & 0 & 0 & 0 & 0 & 0 & \\
\hline Sell & 3 & 0 & 0 & 0 & 0 & 0 & 0 & 0 & 0 & 0 & 14 & 0 & 0 & 0 & 0 & 0 & 0 & 0 & 0 & 0 & 1 & 0 & 0 & 0 & 0 & 0 & & 0 & 0 & \\
\hline BA1* & 60 & 59 & 51 & 36 & 34 & 39 & 53 & 46 & 33 & 0 & 61 & 50 & 29 & 30 & 42 & 40 & 42 & 17 & 0 & 0 & 61 & 59 & 52 & 40 & 38 & 41 & 50 & 524 & 49 & \\
\hline $\mathrm{AA} 1^{*}$ & 0 & 0 & 0 & 0 & 0 & 0 & 0 & 0 & 0 & 0 & 0 & 0 & 0 & 0 & 0 & 0 & 0 & 0 & 0 & 0 & 0 & 0 & 1 & 0 & 0 & 0 & 0 & 0 & 0 & \\
\hline BA1 & 2 & 0 & 0 & 0 & 0 & 0 & 0 & 0 & 0 & 0 & 11 & 0 & 0 & 0 & 0 & 0 & 0 & 0 & 0 & 0 & 5 & \begin{tabular}{|l|} 
\\
\end{tabular} & 0 & 0 & 0 & 0 & 0 & 0 & 0 & \\
\hline $\mathrm{BA} 2$ & 0 & 0 & 0 & 0 & 0 & 0 & 0 & 0 & 0 & 0 & 0 & 0 & 0 & 0 & 0 & 0 & 0 & 0 & 0 & 0 & 0 & \begin{tabular}{|l|} 
\\
\end{tabular} & 0 & 0 & 0 & 0 & 0 & 0 & 0 & \\
\hline BA3 & 0 & 0 & 0 & 0 & 0 & 0 & 0 & 0 & 0 & 0 & 0 & 0 & 0 & 0 & 0 & 0 & 0 & 0 & 0 & 0 & 3 & \begin{tabular}{|l|}
0 \\
\end{tabular} & 0 & 0 & 0 & 0 & 0 & 0 & 0 & \\
\hline BA4 & 0 & 0 & 0 & 0 & 0 & 0 & 0 & 0 & 0 & 0 & 0 & 0 & 0 & 0 & 0 & 0 & 0 & 0 & 0 & 0 & 1 & \begin{tabular}{|l|} 
\\
\end{tabular} & 0 & 0 & 0 & 0 & 0 & 0 & 0 & \\
\hline BA5 & 0 & 0 & 22 & 1 & 0 & 0 & 0 & 0 & 0 & 0 & 0 & 1 & 5 & 0 & 0 & 0 & 0 & 0 & 0 & 0 & 1 & 0 & 21 & 0 & 0 & 0 & 0 & 0 & 0 & \\
\hline BA6 & 1 & 0 & 0 & 1 & 0 & 0 & 0 & 0 & 0 & 0 & 0 & 0 & 0 & 0 & 0 & 0 & 0 & 0 & 0 & 0 & 3 & 1 & 0 & 6 & 0 & 0 & 0 & 0 & 0 & \\
\hline BA7 & 0 & 0 & 4 & 2 & 0 & 0 & 0 & 5 & 9 & 0 & 0 & 0 & 1 & 18 & 13 & 4 & 0 & 0 & 0 & 0 & 1 & 0 & 0 & 0 & 0 & 1 & 0 & 1 & 3 & \\
\hline BA8 & 2 & 0 & 0 & 0 & 0 & 0 & 0 & 0 & 0 & 0 & 0 & 0 & 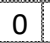 & 0 & 0 & 0 & 0 & 0 & 0 & 0 & 0 & 0 & 0 & 0 & 0 & 0 & & 0 & 0 & \\
\hline BA9 & 0 & 0 & 0 & 0 & 0 & 0 & 0 & 0 & 0 & 0 & 1 & 0 & 0 & 0 & 0 & $c$ & 0 & 0 & 0 & 0 & 1 & 10 & 0 & 0 & 0 & 10 & & 0 & 0 & \\
\hline BA10 & 14 & 0 & 0 & 0 & 0 & 0 & 0 & 0 & 0 & 0 & \begin{tabular}{|l|}
21 \\
\end{tabular} & 1 & U & 0 & 0 & 0 & 0 & 0 & 0 & 0 & 25 & 0 & 0 & 0 & 0 & 0 & & 0 & 0 & \\
\hline $\mathrm{AA1}$ & 0 & 0 & 0 & 0 & 0 & 0 & 0 & 0 & 0 & 0 & 0 & 0 & 0 & 0 & 0 & 0 & 0 & 0 & 0 & 0 & 0 & 0 & 0 & 0 & 0 & 10 & & 0 & 0 & \\
\hline $\mathrm{AA2}$ & 0 & 0 & 0 & 0 & 0 & 0 & 0 & 0 & 0 & 0 & 0 & 0 & 0 & 0 & 0 & 0 & 0 & 0 & 0 & 0 & 0 & 0 & 0 & 0 & 0 & 10 & & 0 & 0 & \\
\hline AA3 & 0 & 0 & 0 & 0 & 0 & 0 & 0 & 0 & 0 & 0 & 0 & 0 & 0 & 0 & 0 & 0 & 0 & 0 & 0 & 0 & 0 & 0 & 0 & 0 & 0 & 0 & & 0 & 0 & \\
\hline $\mathrm{AA} 4$ & 0 & 0 & 0 & 0 & 0 & 0 & 0 & 0 & 0 & 0 & 0 & 0 & 0 & 0 & 0 & 0 & 0 & 0 & 0 & 0 & 0 & 0 & 0 & 0 & 0 & 0 & 0 & 0 & 0 & \\
\hline AA5 & 0 & 0 & 0 & 0 & 0 & 0 & 0 & 0 & 0 & 0 & 0 & 0 & 0 & 0 & 0 & 0 & 0 & 0 & 0 & 0 & 0 & 0 & 0 & 0 & 0 & 0 & 0 & 0 & 0 & \\
\hline $\mathrm{AA6}$ & 0 & 0 & 0 & 0 & 0 & 0 & 0 & 0 & 0 & 0 & 0 & 0 & 0 & 0 & 0 & 0 & 0 & 0 & 0 & 0 & 0 & 0 & 0 & 0 & 0 & 0 & 0 & 0 & 0 & \\
\hline AA7 & 0 & 0 & 0 & $c$ & 0 & 0 & 0 & 0 & 0 & 0 & 0 & 0 & 0 & 0 & 0 & 0 & 0 & 0 & 0 & 0 & 0 & \begin{tabular}{|l|} 
\\
\end{tabular} & 0 & 0 & 0 & 0 & 0 & 0 & 0 & \\
\hline AA8 & 0 & 0 & 0 & 0 & 0 & 0 & 0 & 0 & 0 & 0 & 0 & 0 & 0 & 0 & 0 & 0 & 0 & 0 & 0 & 0 & 0 & 0 & 0 & 0 & $\begin{array}{ll}0 \\
\end{array}$ & 0 & 0 & 0 & 0 & \\
\hline AA9 & 0 & 0 & 0 & 0 & 0 & 0 & 0 & 0 & 0 & 1 & 0 & 0 & 2 & 0 & 2 & 0 & 0 & 0 & 0 & 0 & 0 & 0 & 0 & 0 & 0 & 0 & 0 & 0 & 0 & \\
\hline AA10 & 0 & 0 & 0 & 0 & 0 & 0 & 0 & 0 & 0 & 0 & 0 & 2 & 0 & 0 & 0 & 0 & 0 & 0 & 0 & 0 & 0 & 0 & 0 & 0 & 0 & 0 & 0 & 0 & 0 & \\
\hline $\mathrm{BC} 11$ & 2 & 1 & 4 & 11 & 16 & 9 & 12 & 16 & 8 & 0 & 0 & 7 & 28 & 14 & 12 & 20 & 16 & 1 & 0 & 0 & 6 & 2 & 2 & 7 & 3 & 12 & 8 & 15 & 10 & \\
\hline 3C12 & 4 & 2 & 1 & 7 & 8 & 4 & 5 & 5 & 3 & 3 & 1 & 2 & \begin{tabular}{l|l} 
\\
\end{tabular} & 15 & 7 & 2 & 2 & 0 & 0 & 0 & 5 & 1 & 6 & 4 & 7 & 5 & 4 & 8 & 4 & \\
\hline $\mathrm{BC} 13$ & 1 & 2 & 4 & $c$ & 7 & 14 & 13 & 4 & 0 & 3 & 1 & 0 & 2 & 3 & 8 & 0 & 0 & 0 & 0 & 0 & 3 & 1 & 1 & 1 & 3 & 1 & 3 & 1 & 2 & \\
\hline $\mathrm{BC} 14$ & 1 & 4 & 6 & 4 & 1 & 2 & 1 & 5 & 0 & 0 & 1 & 3 & 4 & 0 & 2 & 1 & 1 & 0 & 1 & 0 & 0 & \begin{tabular}{|l|}
1 \\
\end{tabular} & 1 & 2 & 4 & 2 & 5 & 0 & 2 & \\
\hline $\mathrm{BC15}$ & 1 & 2 & 2 & 2 & 12 & 1 & 2 & 2 & 1 & 0 & 1 & 3 & 6 & 2 & 7 & 9 & 4 & 0 & 0 & 0 & 1 & 3 & 1 & 3 & 4 & 2 & 4 & 1 & 3 & \\
\hline $\mathrm{BC} 16$ & 6 & 3 & 1 & 4 & 3 & 8 & 2 & 4 & 1 & 1 & 1 & 3 & 11 & 3 & 4 & 16 & 3 & 2 & 2 & 0 & 0 & 0 & 2 & 2 & 3 & 7 & 3 & 1 & 2 & 0 \\
\hline $\mathrm{BC} 17$ & 4 & 3 & 5 & 5 & 3 & 2 & 10 & 4 & 3 & 4 & 1 & 4 & 5 & 6 & 5 & 2 & 14 & 1 & 0 & 0 & 0 & 3 & 1 & 4 & 1 & 1 & 16 & 5 & 2 & \\
\hline $\mathrm{BC} 18$ & 1 & 1 & 4 & 9 & 0 & 6 & 4 & 12 & 7 & 1 & 1 & 2 & 2 & 2 & 3 & 7 & 3 & 1 & 0 & 0 & 1 & 1 & 2 & 4 & 4 & 1 & 2 & 7 & 4 & \\
\hline BC19 & 1 & 3 & 5 & 2 & 3 & 0 & 7 & 6 & 2 & 0 & 0 & 0 & 1 & 0 & 0 & 0 & 0 & 2 & 0 & 0 & 0 & 11 & 5 & 4 & 1 & 10 & 3 & 10 & 5 & \\
\hline $\mathrm{BC} 20$ & 0 & 0 & 2 & 4 & 4 & 2 & 1 & 1 & 1 & 0 & 0 & 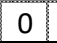 & 0 & 0 & 0 & 0 & 0 & 0 & 0 & 0 & 1 & 1 & 2 & 2 & 0 & 1 & 1 & 1 & 2 & \\
\hline AC11 & 0 & 0 & 0 & 0 & 0 & 0 & 0 & \begin{tabular}{ll|}
0 & \\
\end{tabular} & 0 & 0 & 0 & 0 & 0 & 0 & 0 & 0 & 0 & 0 & 0 & 0 & \begin{tabular}{|l|} 
\\
\end{tabular} & \begin{tabular}{l|}
0 \\
\end{tabular} & 0 & 0 & 0 & 0 & 0 & 0 & 0 & \\
\hline AC12 & 3 & 2 & 0 & 1 & 0 & 0 & 0 & 0 & 0 & 0 & 0 & 0 & 0 & 0 & 0 & 0 & 0 & 0 & 0 & 0 & 1 & 3 & 1 & 2 & 1 & 0 & \begin{tabular}{l|l}
0 & \\
\end{tabular} & \begin{tabular}{|l|l}
0 \\
\end{tabular} & 0 & \\
\hline $\mathrm{AC} 13$ & 1 & 7 & 1 & 0 & 0 & 0 & 0 & 1 & 0 & 0 & 0 & 0 & 0 & 0 & 0 & 0 & 0 & 0 & 1 & 0 & 0 & \begin{tabular}{|l|}
3 \\
\end{tabular} & $\begin{array}{ll}0 \\
\end{array}$ & 0 & 0 & 0 & 0 & 0 & 0 & 0 \\
\hline AC14 & 1 & 4 & 0 & 2 & 0 & 0 & 0 & 0 & 0 & 0 & 0 & 0 & 0 & 0 & 0 & 0 & 0 & 0 & 0 & 0 & 0 & $|0|$ & 1 & 0 & 0 & 0 & 0 & \begin{tabular}{l|l}
0 \\
0
\end{tabular} & 0 & 0 \\
\hline AC15 & 0 & 2 & 0 & 0 & 0 & 0 & 0 & 0 & 0 & 0 & 0 & 0 & 0 & 0 & 0 & 0 & 1 & 0 & 0 & 0 & 0 & 1 & 0 & 1 & 0 & 0 & 0 & 1 & 0 & \\
\hline AC16 & 0 & 3 & 0 & $c$ & 0 & 0 & 0 & 0 & 0 & 0 & 0 & 1 & 0 & 0 & 0 & 0 & 0 & 0 & 0 & 0 & 0 & 0 & 0 & 1 & 0 & 0 & 1 & 0 & 0 & \\
\hline AC17 & 0 & 2 & 1 & 0 & 0 & 0 & 0 & 0 & 0 & 0 & 0 & 0 & 0 & 0 & 0 & 0 & 0 & 0 & 0 & 0 & 2 & 1 & 1 & 0 & 0 & 1 & 0 & 0 & 0 & \\
\hline AC18 & 0 & 0 & 0 & 1 & 0 & 0 & 0 & 0 & 0 & 0 & 0 & 0 & 0 & 0 & 0 & 0 & 0 & 0 & 0 & 0 & 0 & \begin{tabular}{|l|} 
\\
\end{tabular} & 3 & 1 & 0 & 0 & 0 & 0 & 0 & \\
\hline AC19 & 1 & 0 & 0 & 0 & 0 & 0 & 0 & 0 & 0 & 0 & 1 & 0 & 0 & 0 & 0 & 0 & 0 & 0 & 0 & 0 & 0 & 3 & 0 & 0 & 1 & 0 & 0 & 0 & 0 & \\
\hline $\mathrm{AC} 20$ & 10 & 10 & & 0 & & & 0 & 0 & 0 & & & 10 & & & 0 & & 0 & 0 & 0 & & 0 & 1 & 0 & 0 & & 0 & & & & \\
\hline
\end{tabular}

Note: Due to the general absence of relationships involving these explanatory events, BA11 to BA20, AA11 to $\mathrm{AA} 20, \mathrm{BC} 1$ to $\mathrm{BC} 10$ and $\mathrm{AC} 1$ to $\mathrm{AC} 10$ are omitted. 


\section{Panel VI: AA1 to AA10}

\begin{tabular}{|c|c|c|c|c|c|c|c|c|c|c|c|c|c|c|c|c|c|c|c|c|c|c|c|c|c|c|c|c|c|c|}
\hline & \multicolumn{10}{|c|}{ VWW } & \multicolumn{10}{|c|}{ SAP } & \multicolumn{10}{|c|}{ ADS } \\
\hline $\mathrm{AA}$ & 1 & 2 & 3 & 4 & 5 & 6 & 7 & & 9 & 10 & 1 & 2 & & 4 & 5 & & 7 & 8 & 9 & & & 2 & 3 & 4 & & & & & 9 & \\
\hline y* & 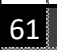 & 36 & 59 & 55 & 46 & 32 & 29 & 40 & 59 & 61 & 61 & 31 & 58 & 53 & 43 & 49 & 39 & 524 & 496 & 61 & 60 & 35 & 575 & 52 & & & 31 & & 60 & \\
\hline II* & & 61 & 60 & 58 & 49 & 21 & 14 & 11 & 0 & & 61 & 60 & 51 & 48 & 50 & 31 & 21 & 10 & 2 & 0 & $61 \Theta$ & 61 & 615 & 59 & 54 & & 18 & & 4 & \\
\hline iy & 1 & 12 & 18 & 10 & 6 & 6 & 5 & & 1 & 5 & 39 & 13 & 5 & 4 & 10 & 4 & 4 & 3 & 0 & 11 & 23 & 4 & 14 & 3 & 5 & & 1 & 0 & 1 & \\
\hline ell & 8 & 59 & 52 & 29 & 9 & 1 & 0 & & 0 & 0 & 51 & 53 & 26 & 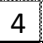 & 0 & 0 & 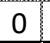 & 0 & & 0 & 50 & 54 & 411 & 15 & 6 & & & 3 & 0 & \\
\hline $1 *$ & 3 & 30 & 53 & 11 & 13 & 19 & 28 & 25 & 11 & 21 & 30 & 38 & 23 & 14 & 11 & 12 & 18 & 114 & 45 & 19 & 4 & 24 & 121 & 12 & 12 & 5 & 202 & 22 & 20 & \\
\hline $11 *$ & 61 & 61 & 61 & 61 & 56 & 55 & 23 & 4 & 0 & 1 & 53 & 61 & 61 & 60 & 44 & 31 & 26 & 5 & 2 & 9 & 60 & 61 & 616 & 61 & 52 & 18 & & 9 & 2 & \\
\hline $\mathrm{A1}$ & 2 & 0 & 0 & 0 & 0 & 0 & 0 & 0 & 0 & 0 & 0 & 0 & 0 & 0 & 0 & 0 & 0 & 0 & 0 & 0 & 1 & 0 & 0 & 0 & 0 & 0 & 0 & 0 & 0 & \\
\hline 2 & 0 & 2 & 3 & 3 & 0 & 1 & 0 & & 0 & 0 & 0 & 1 & & - & 0 & 0 & 0 & 0 & & C & 0 & 0 & 0 & & 0 & & & 0 & 1 & \\
\hline 3 & 0 & 1 & 54 & 1 & 0 & 0 & 0 & & 0 & 0 & 1 & 2 & 39 & 4 & 3 & 0 & 0 & 0 & & 0 & 0 & 0 & 0 & & 0 & 0 & & 1 & 0 & \\
\hline 4 & & & & 18 & 1 & 0 & 1 & 0 & 0 & 0 & & & & 16 & 2 & 0 & 0 & 0 & 0 & 0 & & & & 17 & 0 & 0 & & 0 & 0 & \\
\hline A5 & & & & 9 & 12 & 2 & 0 & 0 & 0 & & & & & 2 & 1 & 0 & 0 & 0 & 0 & & & & & 1 & 2 & 1 & & 0 & 0 & \\
\hline A6 & & & & 15 & 4 & 1 & 1 & 0 & 0 & & & & & 7 & 2 & 1 & 0 & 0 & 0 & & & & & 3 & 0 & & U & 0 & 0 & \\
\hline A7 & & & & & & & 1 & 0 & 0 & & & & & & & & 3 & 0 & 0 & 0 & & & & & & & & 0 & 1 & \\
\hline A8 & & & & & & & 0 & & 0 & & & & & & & & 0 & 0 & 0 & & & & & & & & & 0 & 0 & \\
\hline A9 & & & & & & & 3 & & 0 & & & & & & & & 2 & & 2 & & & & & & & & & & 0 & \\
\hline 10 & & & & & & & 0 & & $C$ & & & & & & & & 0 & 0 & 0 & 12 & & & & & & & & & 0 & \\
\hline A1 & & 61 & 61 & 59 & 7 & 0 & 0 & & 0 & & & 61 & 58 & 21 & 11 & 0 & 0 & 0 & 0 & 4 & 51 & 61 & 614 & & 20 & & & & 0 & \\
\hline 2 & 54 & 61 & 59 & 19 & 5 & 0 & 0 & & 0 & 0 & 39 & 18 & 41 & 18 & 6 & 1 & 0 & 0 & 0 & 0 & 25 & 57 & 571 & 13 & 4 & & & & 0 & \\
\hline 3 & 31 & 60 & 59 & 37 & 6 & 0 & 2 & & 0 & 0 & 6 & 59 & 43 & 48 & 9 & 2 & 1 & 1 & 0 & 0 & 20 & & 563 & 342 & 25 & 4 & 11 & 1 & 0 & \\
\hline 4 & & & & 29 & 51 & 2 & 1 & & 0 & c & & & & 7 & 38 & 2 & 1 & 0 & 1 & 0 & & & & 10 & 37 & 2 & & 0 & 0 & \\
\hline 5 & & & & 44 & 19 & 44 & 3 & 0 & 3 & & & & & 0 & 0 & 25 & 0 & 1 & 3 & & & & & 30 & 7 & 7 & & 0 & 0 & \\
\hline 6 & & & & 12 & 28 & 6 & 42 & 11 & 3 & 14 & & & & 10 & 1 & 0 & 27 & 5 & 1 & & & & & 3 & 30 & & & 0 & 0 & \\
\hline 17 & & & & & & & 0 & 50 & 9 & 7 & & & & & & & 2 & 26 & 4 & & & & & & & & & 55 & 2 & \\
\hline 18 & & & & & & & 0 & 1 & 56 & 4 & & & & & & & 5 & 2 & 37 & 2 & & & & & & & 8 & 1 & 57 & \\
\hline 19 & & & & & & & 6 & 3 & 1 & 59 & & & & & & & 2 & 13 & 1 & 52 & & & & & & & & 26 & 1 & \\
\hline $\mathrm{A} 10$ & & & & & & & 6 & 3 & . & 0 & & & & & & & 3 & 3 & 0 & & & & & & & & 6 & 4 & 1 & \\
\hline $\mathrm{C} 1$ & 61 & 61 & 61 & 59 & 28 & 0 & 1 & 0 & 0 & 0 & 1 & 60 & 61 & 43 & 11 & 1 & 0 & 0 & 0 & 0 & 61 & 61 & 606 & $61<$ & 49 & 4 & 0 & 0 & 0 & \\
\hline C2 & 53 & 57 & 57 & 32 & 0 & 0 & 0 & $c$ & 0 & 0 & 41 & 51 & 51 & 3 & 1 & U & 0 & U & 0 & 0 & 22 & 59 & 57 & 32 & 8 & 0 & & U & 0 & \\
\hline 3 & 57 & 60 & 60 & 49 & 4 & 0 & 0 & 0 & 0 & 0 & 24 & 39 & 48 & 8 & 3 & U & 0 & 0 & 0 & 0 & 32 & 51 & 59 & 40 & 8 & U & U & 0 & 0 & \\
\hline 4 & & & & 34 & 4 & 0 & . & C & 0 & C & & & & 4 & 8 & 3 & 0 & 0 & 0 & 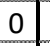 & & & & 33 & & 0 & & 0 & 0 & \\
\hline 5 & & & & 13 & 0 & 0 & 0 & & 0 & C & & & & 5 & 7 & 7 & 2 & 0 & 0 & 0 & & & & 16 & 8 & 5 & 10 & 1 & 0 & \\
\hline C6 & & & & 27 & 0 & 0 & 0 & & 0 & c & & & & 12 & 4 & 3 & 4 & 1 & 0 & 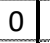 & & & & 18 & 0 & 0 & 3 & 1 & 0 & \\
\hline 77 & & & & & & & 2 & & 2 & c & & & & & & & 1 & 4 & 0 & 0 & & & & & & & & 3 & 5 & 0 \\
\hline 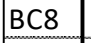 & & & & & & & 1 & & 2 & 2 & & & & & & & $\ldots$ & 4 & 0 & 1 & & & & & & & & 0 & 0 & \\
\hline & & & & & & & 2 & & 0 & c & & & & & & & 0 & 3 & 0 & 1 & & & & & & & & 0 & 1 & \\
\hline 4 & & & & & & & 1 & & 0 & 0 & & & & & & & 6 & 1 & 6 & 1 & & & & & & & & & 0 & \\
\hline C1 & & 1 & 61 & 28 & 21 & 17 & & & 1 & 59 & 61 & 43 & 58 & 32 & 14 & 21 & 7 & 13 & 0 & 57 & 61 & 59 & & 29 & 27 & & & 39 & 14 & \\
\hline 2 & & I & 60 & 35 & 2 & 1 & & 1 & 21 & 0 & 32 & 42 & 58 & 34 & 10 & 6 & 13 & 2 & 50 & 0 & 41 & 55 & 614 & 47 & 5 & 5 & & 37 & 58 & \\
\hline C3 & 1 & & 59 & 6 & 26 & 4 & 12 & $\varepsilon$ & 0 & 59 & 10 & 35 & 61 & 59 & 42 & 2 & 2 & 2 & 0 & 4 & 24 & & 316 & 61 & 17 & & 10 & 11 & 1 & 6 \\
\hline CC4 & & & & 46 & 51 & 18 & 4 & 1 & 1 & 0 & & & & 61 & 44 & 48 & 1 & 1 & 0 & - & & & & 34 & 54 & & 4 & 0 & 1 & \\
\hline C5 & & & & 53 & 57 & 9 & 4 & 1 & 10 & 0 & & & & 41 & 61 & 27 & 6 & 2 & 1 & 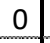 & & & & 58 & 57 & 1 & 6 & 1 & 12 & 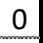 \\
\hline 66 & & & & 58 & 9 & 57 & 0 & 0 & 0 & C & & & & 49 & 41 & 61 & 14. & 3 & 0 & 1 & & & & 58 & & & 19 & 1 & 0 & \\
\hline C7 & & & & & & & 46 & 6 & 0 & 0 & & & & & & & 39 & 7 & 1 & 0 & & & & & & & & 11 & 3 & 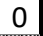 \\
\hline CC8 & & & & & & & 1 & 43 & 14 & 5 & & & & & & & 12 & 31 & 0 & 0 & & & & & & & & 42 & 14 & \\
\hline $\mathrm{CO}$ & & & & & & & 0 & 4 & 21 & 3 & & & & & & & 2 & 4 & 7 & 1 & & & & & & & 1 & 2 & 16 & \\
\hline 10 & & & & & & & 3 & & 5 & 11 & & & & & & & 1 & 2 & 2 & 1 & & & & & & & 6 & 0 & 4 & \\
\hline
\end{tabular}




\section{Panel VII: AA11 to AA20}

\begin{tabular}{|c|c|c|c|c|c|c|c|c|c|c|c|c|c|c|c|c|c|c|c|c|c|c|c|c|c|c|c|c|c|c|}
\hline & \multicolumn{10}{|c|}{ BMW } & \multicolumn{10}{|c|}{ SAP } & \multicolumn{10}{|c|}{ ADS } \\
\hline $\mathrm{AA}$ & 11 & & $13:$ & & & & & 18 & & & 11 & 12 & & 14 & & & & & 19 & & 11 & 12 & 13 & & & & & & & \\
\hline Buy* & \begin{tabular}{|l|}
43 \\
\end{tabular} & 33 & 29 & 39 & 45 & 31 & 10 & 3 & 0 & 0 & 47 & 30 & 47 & 47 & 46 & 33 & 5 & 0 & 0 & 0 & 45 & 27 & 34 & 37 & 40 & & 27 & 4 & 3 & \\
\hline Sell* & 0 & 0 & 2 & 8 & 3 & 2 & 2 & 0 & 0 & 8 & 1 & 1 & 25 & 12 & 1 & 0 & 0 & 0 & 0 & 0 & 0 & 1 & 0 & \begin{tabular}{|l|} 
\\
\end{tabular} & 0 & 4 & 1 & 2 & 2 & \\
\hline Buy & 4 & 1 & 0 & 0 & 0 & 0 & ) & 0 & 0 & 0 & 7 & 0 & 0 & 0 & 0 & & & & 0 & & 7 & 0 & 0 & 0 & 0 & 0 & & 0 & 0 & \\
\hline Sell & 0 & 0 & 0 & 0 & 0 & $\underline{0}$ & & 0 & $\underline{0}$ & & 0 & 0 & 0 & 0 & 0 & & & & 0 & & 0 & 0 & 0 & 0 & 0 & 0 & & 0 & 0 & \\
\hline BA1* & 6 & 8 & 7 & 12 & 2 & 1 & 0 & 0 & 0 & 0 & 12 & 5 & 2 & 12 & 16 & & 0 & & 0 & & 4 & 12 & 9 & 8 & 7 & 10 & 3 & 0 & 0 & \\
\hline AA1* & 0 & 2 & 3 & 8 & 16 & 23 & 17 & 1 & 0 & 0 & 3 & 1 & 7 & 23 & 6 & 0 & 0 & & 0 & 0 & 0 & 0 & 5 & 4 & 10 & 14 & 13 & 13 & 3 & \\
\hline $\mathrm{BC} 1$ & 0 & 0 & 0 & 0 & 0 & 0 & 0 & 0 & 0 & 0 & 0 & 0 & 0 & 0 & 0 & 0 & 0 & Q & 0 & 0 & 0 & 0 & 0 & 0 & 0 & 0 & 0 & 0 & 0 & \\
\hline $\mathrm{BC} 2$ & 0 & 0 & 0 & 0 & 0 & 0 & 0 & 0 & 0 & 0 & 0 & 0 & 0 & 0 & 0 & 0 & 0 & 0 & 0 & 0 & 0 & 0 & 0 & 0 & 0 & 0 & 0 & & 0 & \\
\hline $\mathrm{BC} 3$ & 0 & 0 & 0 & 0 & 0 & 0 & 0 & 0 & 0 & 0 & 0 & 0 & 0 & 0 & 0 & 0 & 0 & 0 & 0 & 0 & 0 & 0 & 0 & 0 & 0 & 0 & 0 & & 0 & \\
\hline $\mathrm{BC} 4$ & 0 & 0 & 0 & 0 & 0 & 0 & 0 & 0 & 0 & 0 & 0 & 0 & 0 & 0 & 0 & 0 & 0 & 0 & 0 & U & 0 & 0 & 0 & 0 & 0 & 0 & 0 & 0 & 0 & \\
\hline BC5 & 0 & 0 & 0 & 0 & 0 & 0 & 0 & 0 & 0 & 0 & 0 & 0 & 0 & 0 & 0 & 0 & 0 & 0 & 0 & 0 & 0 & 0 & 0 & 0 & 0 & 0 & 0 & 0 & 0 & \\
\hline BC6 & 0 & 0 & 0 & 0 & 0 & 0 & 0 & 0 & 0 & 0 & 0 & 0 & 0 & 0 & 0 & 0 & 0 & 0 & 0 & 0 & 0 & 0 & 0 & 0 & 0 & 0 & 0 & 0 & 0 & \\
\hline BC7 & 0 & 0 & 0 & 0 & 0 & 0 & 0 & 0 & 0 & 0 & 0 & 0 & 0 & 0 & 0 & 0 & 0 & 0 & 0 & 0 & 0 & 0 & 0 & 0 & 0 & 0 & 0 & 0 & 0 & \\
\hline BC8 & 0 & 0 & 2 & 0 & 1 & 0 & 0 & 0 & 0 & 0 & 0 & 0 & 0 & 0 & 0 & 0 & 0 & 0 & 0 & 0 & 0 & 0 & 0 & 0 & 0 & 0 & 0 & 0 & 0 & \\
\hline BC9 & 1 & 1 & 0 & 1 & 0 & 1 & 0 & 0 & 0 & 0 & 1 & 0 & 1 & 0 & 0 & 0 & 1 & 0 & 0 & J & 0 & 0 & 1 & 1 & 0 & 0 & 0 & 0 & 0 & \\
\hline $\mathrm{BC} 10$ & 3 & 2 & 2 & 6 & 1 & 0 & 1 & 1 & 0 & 0 & 1 & 2 & 1 & 1 & 5 & 4 & 0 & 0 & 0 & 0 & 0 & 4 & 4 & 1 & 1 & 1 & 0 & 0 & 0 & \\
\hline BC11 & 6 & 5 & 3 & 8 & 0 & 2 & 0 & 0 & 0 & 0 & 6 & 1 & 0 & 1 & 5 & 6 & 0 & 0 & 0 & 0 & 2 & 4 & 4 & 7 & 1 & 3 & 0 & 0 & 0 & \\
\hline $\mathrm{BC} 12$ & 4 & 6 & 3 & 11 & 5 & 2 & 4 & 0 & 0 & 0 & 2 & 1 & 0 & 0 & 0 & 0 & 1 & 0 & 0 & 0 & 0 & 2 & 7 & 3 & 3 & 4 & & 0 & 0 & \\
\hline $\mathrm{BC} 13$ & 1 & 2 & 0 & 1 & 2 & 0 & 0 & 1 & 0 & 1 & 0 & 0 & 0 & 0 & 0 & 0 & 0 & 0 & 0 & 1 & 0 & 2 & 0 & 0 & 0 & 0 & & 0 & 0 & \\
\hline $\mathrm{BC} 14$ & 0 & 2 & 2 & 2 & 0 & 0 & U & 1 & 1 & 0 & 0 & 0 & 0 & 0 & 0 & 0 & 0 & 0 & 0 & 0 & 0 & 1 & 0 & 0 & 1 & 0 & 0 & 1 & 0 & \\
\hline $\mathrm{BC} 15$ & 0 & 1 & 1 & 2 & 0 & 1 & 1 & 1 & 0 & 0 & 3 & 0 & 0 & 0 & 0 & 0 & 0 & 0 & 0 & 0 & 0 & 1 & 0 & 1 & 0 & 1 & 0 & 0 & 0 & \\
\hline $\mathrm{BC} 16$ & 1 & 5 & 2 & 3 & 3 & 1 & 1 & 4 & C & 0 & 6 & 1 & 1 & 2 & 3 & 3 & 1 & U & 2 & U & 1 & 1 & 1 & 4 & 0 & 0 & 0 & 1 & 0 & \\
\hline $\mathrm{BC} 17$ & 2 & 5 & 4 & 8 & 2 & 0 & 3 & 0 & 0 & $\perp$ & 2 & 3 & 0 & 1 & 5 & 2 & 2 & U & 0 & 0 & 0 & 2 & 1 & 2 & 0 & 1 & 1 & 1 & 0 & \\
\hline $\mathrm{BC} 18$ & 3 & 6 & 3 & 2 & 1 & 0 & & 2 & 0 & 0 & 0 & 0 & 0 & 1 & 1 & 2 & 0 & U & 0 & 0 & 0 & 0 & 0 & 3 & 2 & 0 & 0 & 0 & 0 & \\
\hline $\mathrm{BC} 19$ & 4 & 3 & 1 & 2 & 2 & $c$ & & 0 & 0 & 0 & 0 & 0 & 0 & 0 & 0 & 0 & 0 & U & 0 & 0 & 0 & 2 & 0 & 3 & 1 & 0 & 1 & 0 & 1 & \\
\hline $\mathrm{BC} 20$ & 2 & 1 & 2 & 2 & 0 & 0 & 0 & 0 & 0 & 0 & 0 & 0 & 0 & 0 & 0 & 0 & 0 & 0 & 0 & U & 0 & 0 & 0 & 1 & 0 & 0 & 0 & 0 & 0 & \\
\hline AC1 & 0 & 0 & 0 & 0 & 0 & 0 & 0 & 0 & 0 & 0 & 0 & 0 & 0 & 0 & 0 & 0 & 0 & 0 & 0 & 0 & 1 & 0 & 0 & 1 & 0 & 0 & 0 & 0 & 0 & \\
\hline $\mathrm{AC2}$ & 0 & 0 & 0 & 0 & 17 & 0 & 0 & 0 & 0 & 0 & 0 & 0 & 0 & 0 & 6 & 0 & 0 & 0 & 0 & 0 & 0 & 0 & 0 & 0 & 24 & 0 & 0 & 0 & 0 & \\
\hline AC3 & 0 & 1 & 0 & 0 & 1 & 19 & 0 & 0 & 0 & 0 & 0 & 0 & 0 & 0 & 0 & 0 & 0 & 0 & 0 & 0 & 0 & 1 & 0 & 1 & 3 & 30 & 1 & 0 & 0 & \\
\hline AC4 & 56 & 0 & 0 & 0 & 0 & 1 & 7 & 0 & 0 & 0 & 0 & 0 & 0 & 0 & 0 & 0 & 0 & 0 & 0 & 0 & 54 & 0 & 0 & 0 & 0 & 0 & 6 & 0 & 0 & \\
\hline AC5 & 0 & 11 & 0 & 0 & 0 & 0 & 0 & 0 & 0 & 0 & 0 & 0 & 0 & 0 & 0 & 0 & 0 & 0 & 0 & 0 & 0 & 15 & 0 & 0 & 0 & 0 & 0 & 0 & 0 & \\
\hline AC6 & 0 & 1 & 1 & 0 & 0 & 0 & 0 & 0 & 0 & 0 & 1 & 2 & 3 & 0 & 0 & 0 & 0 & 0 & 0 & 0 & 0 & 0 & 2 & 0 & 0 & 0 & 0 & 0 & 0 & \\
\hline AC7 & 1 & 0 & 3 & 2 & 0 & 1 & 0 & 0 & 0 & 0 & 0 & 0 & 0 & 0 & 0 & 0 & 0 & 0 & 0 & 0 & 7 & 0 & 2 & 5 & 3 & 0 & 0 & 0 & 0 & \\
\hline AC8 & 0 & 0 & 0 & 0 & 0 & 0 & 0 & 0 & 0 & 0 & 0 & 1 & 0 & 0 & 0 & 0 & 0 & 0 & 10 & 0 & 1 & 3 & 0 & 0 & 0 & 0 & 1 & 0 & 0 & \\
\hline AC9 & 4 & 0 & 2 & 0 & 2 & 0 & 0 & 0 & 0 & 0 & 0 & 1 & 2 & 1 & 0 & 0 & 0 & 0 & 0 & 0 & 1 & 0 & 0 & 1 & 0 & 0 & 1 & 0 & 0 & \\
\hline $\mathrm{AC} 10$ & 2 & 2 & 2 & 5 & 6 & 1 & 0 & 0 & 0 & 0 & 0 & 2 & 5 & 3 & 0 & 0 & 0 & 0 & 0 & 0 & 0 & 2 & 0 & 1 & 1 & 0 & 1 & 1 & 0 & \\
\hline AC11 & 0 & 3 & 3 & 2 & 6 & 20 & 14 & 0 & 0 & 0 & 6 & 2 & 9 & 37 & 22 & 0 & 0 & 0 & 0 & 0 & 0 & 0 & 1 & 1 & 4 & 15 & 17 & 20 & 12 & 4 \\
\hline $\mathrm{ACl12}$ & 0 & 7 & 3 & 2 & 5 & 1 & 0 & 1 & 0 & 5 & 0 & 1 & 11 & 7 & 0 & 0 & 0 & 0 & 0 & 0 & 0 & 4 & 4 & 0 & 3 & 1 & 1 & 1 & 1 & \\
\hline $\mathrm{AC} 13$ & 0 & 1 & 7 & 10 & 17 & 11 & 1 & 0 & 0 & 1 & 0 & 1 & 5 & 1 & 2 & 0 & 0 & 0 & 0 & 0 & 0 & 0 & 9 & 1 & 1 & 0 & 2 & 0 & 0 & \\
\hline AC14 & 0 & 2 & 1 & 11 & 8 & & 0 & 0 & 0 & 1 & 1 & 0 & 0 & 4 & 0 & 0 & 0 & 0 & 0 & 0 & 0 & 0 & 7 & 6 & 1 & 1 & 0 & 2 & 0 & \\
\hline AC15 & 0 & 1 & 0 & 0 & 23 & 0 & 0 & 0 & 0 & 2 & 0 & 2 & 1 & 0 & 17 & 0 & 0 & 0 & 0 & 0 & 0 & 0 & 0 & 0 & 14 & 1 & 1 & 0 & 0 & \\
\hline AC16 & 0 & 0 & 2 & 0 & 1 & 25 & 0 & 0 & 0 & 1 & 1 & 13 & 1 & 4 & 1 & 23 & 0 & 0 & 0 & 0 & 0 & 1 & 0 & 0 & 2 & 14 & 1 & 0 & 0 & 2 \\
\hline AC17 & 0 & 0 & 3 & 0 & 2 & 1 & 8 & 0 & 1 & 0 & 3 & 9 & 4 & 1 & 0 & 0 & 4 & 0 & 0 & 1 & 0 & 0 & 1 & 1 & 4 & 1 & 11 & 0 & 0 & 3 \\
\hline$A C 18$ & 0 & 3 & 11] & 10 & 2 & 6 & 1 & 4 & 0 & 1 & 0 & 0 & 2 & 1 & 0 & 0 & 0 & 0 & 0 & 1 & 0 & 1 & 5 & 2 & 2 & 2 & 1 & 3 & 0 & 1 \\
\hline $\mathrm{AC} 19$ & 0 & 0 & 2 & 15 & 3 & 3 & 2 & 0 & 6 & 2 & 0 & 0 & 0 & 0 & 0 & 0 & 0 & 0 & 0 & 0 & 0 & 1 & 6 & 6 & 2 & 1 & 3 & 3 & 2 & \\
\hline AC20 & & & & & & & & & 0 & & & 0 & & & & & & & & & & & & & & & & & & \\
\hline
\end{tabular}




\section{Panel VIII: AC1 to AC10}

\begin{tabular}{|c|c|c|c|c|c|c|c|c|c|c|c|c|c|c|c|c|c|c|c|c|c|c|c|c|c|c|c|c|c|c|c|}
\hline & \multicolumn{10}{|c|}{ BMW } & \multicolumn{11}{|c|}{ SAP } & \multicolumn{10}{|c|}{ ADS } \\
\hline $\mathrm{AC}$ & 1 & 2 & 3 & 4 & 5 & 6 & 7 & 8 & 9 & 10 & 1 & 2 & 3 & 4 & & 6 & 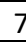 & & & & & & 2 & 3 & 4 & & & 7 & 8 & & \\
\hline dy* & 61 & 61 & 61 & 60 & 59 & 61 & 59 & 48 & 43 & 10 & 61 & 61 & 61 & 61 & 61 & 60 & 5. & & & 4 & 24 & 61 & 61 & 61 & 60 & 60 & 59 & & 47 & & \\
\hline$\| *$ & 1 & 35 & 47 & 54 & 43 & 33 & 21 & 25 & 31 & 51 & 26 & 37 & 40 & 51 & 57 & 31 & 2 & 2 & & 5 & 53 & 16 & 51 & 50 & 48 & 46 & 32 & 23 & 29 & 32 & \\
\hline dy & 9 & 61 & 58 & 50 & 4 & 44 & 23 & 30 & 16 & 0 & 61 & 58 & 59 & 47 & 40 & 32 & $1 !$ & & & 0 & 0 & 60 & 53 & 54 & 37 & & 33 & 23 & 19 & 6 & \\
\hline ell & 13 & 28 & 18 & 18 & 15 & 3 & 4 & 1 & 1 & 0 & 10 & 14 & 11 & 14 & 10 & 2 & 0 & & & 0 & 0 & 4 & 12 & 9 & 5 & 1 & 9 & 0 & 0 & 0 & \\
\hline$A 1 *$ & 61 & 61 & 61 & 59 & 54 & 36 & 9 & 3 & 1 & 0 & 61 & 60 & 51 & 48 & 42 & 23 & 18 & & & 2 & 0 & 61 & 61 & 61 & 61 & 58 & 58 & 34 & 10 & 4 & \\
\hline $\mathrm{Al}^{*}$ & 7 & 61 & 60 & 61 & 60 & 61 & 61 & 60 & 61 & 61 & 10 & 61 & 59 & 59 & 57 & 61 & 6 & 10 & & 6 & 60 & 7 & 59 & 59 & 61 & 0 & 61 & 61 & 59 & 59 & 6 \\
\hline 11 & 61 & 61 & 61 & 37 & 3 & 0 & 0 & 0 & 0 & 0 & 61 & 60 & 50 & 10 & 1 & 0 & $c$ & & & 0 & 0 & 61 & 60 & 60 & 29 & 11 & 4 & 0 & 0 & 0 & \\
\hline 12 & 51 & 57 & 50 & 15 & 1 & 0 & 0 & 0 & 0 & 0 & 50 & 40 & 22 & 4 & & & & & & o & 0 & 52 & 51 & 49 & 20 & 10 & 4 & 0 & 0 & & \\
\hline 43 & 26 & 53 & 54 & 3 & 0 & 0 & 0 & 0 & 0 & 0 & 18 & 39 & 34 & 3 & 0 & & C & & & 0 & & 5 & 45 & 56 & 22 & & 0 & 0 & 0 & 0 & \\
\hline 14 & & & & 1 & 0 & 0 & 0 & 0 & 0 & 0 & & & & 3 & $c$ & & $c$ & & & & & & & & 6 & & & 0 & 0 & 0 & \\
\hline A5 & & & & 2 & 1 & 0 & 0 & 0 & 0 & 0 & & & & 1 & 2 & U & $c$ & & & & & & & & 2 & & 0 & 0 & ) & 0 & \\
\hline $\mathrm{A} 6$ & & & & 1 & 0 & 0 & 0 & 0 & 0 & 0 & & & & 7 & & 2 & $c$ & & & & & & & & 0 & 0 & 0 & 0 & I & 0 & \\
\hline A7 & & & & & & & 0 & 0 & 0 & 0 & & & & & & & $c$ & & & & & & & & & & & 0 & 0 & 0 & \\
\hline A8 & & & & & & & 0 & 0 & 1 & 1 & & & & & & & 0 & & & & & & & & & & & 0 & 0 & 0 & \\
\hline 49 & & & & & & & 1 & 0 & 0 & 0 & & & & & & & 1 & & & & & & & & & & & 0 & ) & 0 & \\
\hline 0 & & & & & & & 0 & 0 & $c$ & 0 & & & & & & & C & & & & & & & & & & & & & 0 & \\
\hline A1 & 54 & 56 & 21 & 37 & 9 & 2 & 1 & 0 & 0 & 0 & 60 & 28 & 19 & 22 & 15 & 3 & 0 & & & & 0 & 48 & 26 & 1 & 4 & & 1 & 0 & ) & 0 & \\
\hline $\mathrm{A} 2$ & 0 & 39 & 2 & 6 & 14 & 5 & 11 & 1 & 0 & 0 & 0 & 46 & 5 & 5 & 6 & 4 & 1 & & & U & 0 & 1 & 26 & 2 & 2 & & 4 & & & 0 & \\
\hline 13 & 1 & 0 & 41 & 1 & 0 & 2 & 0 & 3 & 0 & 0 & 0 & 1 & 55 & 10 & 1 & 2 & 1 & & & U & 1 & 0 & 0 & 29 & 4 & & & 4 & & 5 & \\
\hline 44 & & & & 16 & 0 & 2 & 0 & 8 & 6 & 0 & & & & 59 & 1 & 0 & C & & & 0 & & & & & 10 & & & 0 & 2 & 8 & \\
\hline A5 & & & & 1 & 37 & 2 & 0 & 0 & 10 & 4 & & & & 3 & 60 & 0 & c & & & 1 & & & & & 1 & & 0 & 0 & 1 & 1 & \\
\hline A6 & & & & 0 & 0 & 27 & 0 & 0 & 0 & & & & & 0 & 3 & 46 & $c$ & & & 1 & & & & & 2 & 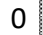 & 11 & 1 & 0 & 1 & \\
\hline 47 & & & & & & & 2 & 4 & 0 & 2 & & & & & & & & & & 1 & & & & & & & & 2 & 9 & 1 & \\
\hline 18 & & & & & & & 0 & 3 & 2 & 7 & & & & & & & & & & & & & & & & & & 0 & 1 & 2 & \\
\hline A9 & & & & & & & 0 & & 3 & & & & & & & & & & & & & & & & & & & 0 & 0 & 1 & \\
\hline 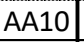 & & & & & & & 0 & & 0 & & & & & & & & 1 & & & & & & & & & & & 0 & 0 & 0 & \\
\hline 1 & 2 & 35 & 30 & 48 & 16 & 1 & 5 & 1 & 0 & 2 & 1 & 19 & 26 & 34 & 10 & 2 & & & & & & & 9 & 5 & 24 & 12 & 5 & 0 & 0 & 0 & \\
\hline$C 2$ & 0 & 10 & 6 & 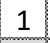 & 0 & 0 & 0 & 0 & 0 & c & 0 & 11 & 14 & 9 & 10 & 0 & 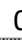 & & & & & & 1 & 4 & 1 & 3 & U & 0 & 0 & 0 & \\
\hline 3 & 4 & 18 & 39 & 11 & 5 & 5 & 0 & 1 & 0 & 3 & 3 & 16 & 27 & 20 & 9 & 1 & $c$ & & & & & & 16 & 17 & 5 & 3 & 3 & 0 & 1 & 0 & \\
\hline C4 & & & & 18 & 15 & 0 & 0 & 0 & 0 & $c$ & & & & 31 & 21 & 6 & C & & & U & & & & & 5 & 8 & 1 & 0 & 0 & 0 & ( \\
\hline $3 \mathrm{CS}$ & & & & 13 & 9 & 1 & 0 & 0 & 0 & 1 & & & & 18 & 16 & 1 & $c$ & & & U & & & & & 7 & 3 & 2 & 0 & 0 & 1 & \\
\hline $3 \mathrm{C} 6$ & & & & 5 & 4 & 1 & 0 & 0 & 1 & 0 & & & & 15 & 14 & 8 & C & & & U & & & & & 5 & 3 & 0 & 0 & 0 & 0 & $c$ \\
\hline C7 & & & & & & & 0 & 0 & 0 & $c$ & & & & & & & 1 & & & 0 & & & & & & & & 10 & 0 & 0 & 2 \\
\hline & & & & & & & $c$ & & 1 & 1 & & & & & & & $c$ & & & 2 & & & & & & & & 10 & 0 & 1 & \\
\hline Cy & & & & & & & 0 & 0 & 1 & 3 & & & & & & & 1 & & & 2 & & & & & & & & 0 & J & 1 & 2 \\
\hline 10 & & & & & & & 2 & & 2 & 1 & & & & & & & $\varepsilon$ & & & 4 & & & & & & & & 1 & 4 & 4 & \\
\hline$C$ & 61 & 61 & 61 & & 45 & 3 & 4 & 0 & 0 & 0 & 61 & 61 & 60 & 54 & 29 & 5 & C & & & 0 & 0 & 61 & 61 & 61 & 59 & 5 & & 6 & 4 & 1 & \\
\hline$A C$ & 60 & 61 & 61 & & 12 & 1 & 0 & 0 & 0 & 0 & 1 1) & 61 & 60 & 43 & 13 & 4 & C & & & 0 & 0 & 60 & 60 & 61 & 38 & 37 & 2 & 1 & 1 & 0 & \\
\hline АC3 & 54 & 61 & 61 & & 51 & 31 & 3 & 0 & 0 & 0 & 31 & 60 & 55 & 44 & 46 & 7 & 4 & & & 0 & 0 & & & 61 & 54 & 46 & & 10 & 0 & 0 & \\
\hline AC4 & & & & 45 & 47 & 46 & 27 & 2 & 0 & 0 & & & & 7 & 32 & 20 & 1 & & & 1 & & & & & 38 & 46 & 47 & 37 & 7 & 0 & \\
\hline & & & & 44 & 22 & 45 & 21 & 6 & 0 & 1 & & & & 18 & 2 & 21 & 7 & 3 & & 5 & & & & & 43 & 18 & 53 & 38 & 14 & 0 & \\
\hline AC6 & & & & 26 & 39 & 25 & 47 & 21 & 1 & 0 & & & & 6 & 20 & 8 & 2 & & & 1 & & & & & 21 & 24 & 2 & 37 & 24 & 5 & 0 \\
\hline$C 7$ & & & & & & & 32 & 20 & 3 & 3 & & & & & & & 1 & 12 & & y & & & & & & & & 7 & 27 & 17 & 4 \\
\hline $\mathrm{AC} 8$ & & & & & & & 22 & 2 & 9 & 9 & & & & & & & $\varepsilon$ & & & 11 & 2 & & & & & & & 29 & 1 & 17 & 1 \\
\hline & & & & & & & 5 & 12 & 1 & 3 & & & & & & & 2 & & & 1 & 5 & & & & & & & 3 & 8 & 0 & 6 \\
\hline 10 & & & & & & & 21 & 17 & & & & & & & & & & & & & & & & & & & & & & & \\
\hline
\end{tabular}




\section{Panel IX: AC11 to AC20}

\begin{tabular}{|c|c|c|c|c|c|c|c|c|c|c|c|c|c|c|c|c|c|c|c|c|c|c|c|c|c|c|c|c|c|c|}
\hline & \multicolumn{10}{|c|}{ BMW } & \multicolumn{10}{|c|}{ SAP } & \multicolumn{10}{|c|}{ ADS } \\
\hline$\overline{A C}$ & 11 & \begin{tabular}{|l|}
12 \\
\end{tabular} & & \begin{tabular}{|l|l|}
14 \\
\end{tabular} & 15 & & $\mid \overline{17}$ & 18 & \begin{tabular}{|l|}
19 \\
\end{tabular} & & 11 & \begin{tabular}{|l|}
12 \\
\end{tabular} & \begin{tabular}{|l|}
13 \\
\end{tabular} & 14 & & & \begin{tabular}{l|l}
17 & 1 \\
\end{tabular} & 18 & 19 & & \begin{tabular}{|l|l}
11 \\
\end{tabular} & \begin{tabular}{|l|}
12 \\
\end{tabular} & \begin{tabular}{l|l}
13 \\
\end{tabular} & \begin{tabular}{|l|}
14 \\
\end{tabular} & & \begin{tabular}{|l|l}
16 & 1 \\
\end{tabular} & & \begin{tabular}{l|l}
18 & 1 \\
\end{tabular} & & \\
\hline Buy* & 8 & 10 & \begin{tabular}{l|l}
12 \\
\end{tabular} & \begin{tabular}{|l|}
11 \\
\end{tabular} & 2 & 0 & 0 & $\begin{array}{ll}0 \\
\end{array}$ & 0 & 0 & 15 & 5 & \begin{tabular}{l|l}
0 & \\
\end{tabular} & 0 & 0 & $\overline{0}$ & 0 & 0 & 0 & 0 & 2 & 1 & $\begin{array}{l}11 \\
\end{array}$ & \begin{tabular}{|l|}
11 \\
\end{tabular} & 0 & $\overline{0}$ & $\overline{0}$ & 0 & $\overline{0}$ & 0 \\
\hline Sell* & 52 & 55 & 45 & 39 & 42 & 49 & 35 & 28 & 15 & 6 & 44 & 48 & 33 & 39 & 31 & 46 & 432 & 24 & 5 & 0 & 60 & 49 & 40 & 37 & 35 & 48 & & \begin{tabular}{l|l}
28 & 1
\end{tabular} & 13 & \\
\hline Buy & 0 & $\begin{array}{ll}0 & \end{array}$ & 0 & 0 & 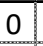 & 0 & 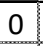 & 21 & 0 & 0 & 0 & 0 & 0 & 0 & 0 & 0 & 0 & \begin{tabular}{l|l|} 
\\
\end{tabular} & 0 & 0 & 1 & 1 & 1 & \begin{tabular}{|l|l}
0 & \\
\end{tabular} & 1 & 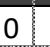 & 0 & 0 & 0 & \\
\hline Sell & 4 & 0 & 1 & 0 & 0 & 0 & 0 & 0 & 0 & 0 & 0 & 0 & 0 & 0 & 0 & 1 & 0 & 4 & $\underline{0}$ & 0 & 1 & 0 & 0 & 0 & 0 & 0 & & $\underline{0}$ & 0 & \\
\hline BA1* & 0 & 0 & 0 & 0 & 4 & 0 & 0 & 0 & 0 & 0 & 1 & 0 & 0 & 0 & 0 & 0 & 0 & 0 & 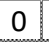 & 0 & 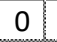 & 0 & 0 & 0 & 0 & 0 & & 0 & 0 & \\
\hline AA1* & & 59 & 42 & 30 & 29 & 325 & 54 & 48 & 35 & 0 & ) & 42 & 32 & 22 & 41 & 34 & 422 & 21 & 2 & 0 & 61 & 58 & 49 & 37 & $40 \angle$ & 415 & 505 & 57[ & & \\
\hline BA1 & 0 & 0 & 0 & 0 & 0 & 0 & 0 & 0 & 0 & 0 & 0 & 0 & 0 & 0 & 0 & 0 & 0 & 0 & 0 & 0 & 0 & 0 & 0 & 0 & 0 & 0 & 0 & 0 & 0 & \\
\hline $\mathrm{BA} 2$ & 0 & 0 & 0 & 0 & 0 & 0 & 0 & 0 & 0 & 0 & 0 & 0 & 0 & 0 & 0 & 0 & 0 & 0 & 0 & 0 & 0 & 0 & 0 & 0 & 0 & 0 & 0 & 0 & 0 & \\
\hline $\mathrm{BA3}$ & 0 & 0 & 0 & 0 & 0 & 0 & 0 & 0 & 0 & 0 & 0 & 0 & 0 & 0 & 0 & 0 & 0 & 0 & 0 & 0 & 0 & 0 & 0 & 0 & 0 & 0 & & 0 & 0 & \\
\hline BA4 & 0 & 0 & 0 & 0 & 0 & 0 & 0 & 0 & 0 & 0 & 0 & 0 & 0 & 0 & 0 & 0 & 0 & 0 & 0 & 0 & 0 & \begin{tabular}{|l|} 
\\
\end{tabular} & 0 & 0 & 0 & 0 & & 0 & 0 & \\
\hline BA5 & 0 & 0 & 0 & 0 & 0 & 0 & 0 & 0 & 0 & 0 & 0 & 0 & 0 & 0 & 0 & 0 & 0 & 0 & 0 & 0 & 0 & 0 & 0 & 0 & 0 & 0 & 0 & 0 & 0 & 0 \\
\hline BA6 & 0 & 0 & 0 & 0 & 0 & 0 & 0 & 0 & 0 & 0 & 0 & 0 & 0 & 0 & 0 & 0 & 0 & 0 & 0 & 0 & 0 & 0 & 0 & 0 & 0 & 0 & 0 & 0 & 0 & 0 \\
\hline BA7 & 0 & 0 & 0 & 0 & 0 & 0 & 0 & 0 & 0 & 0 & 0 & 0 & 0 & 0 & 0 & 0 & 0 & 0 & 0 & 0 & 0 & 0 & 0 & 0 & 0 & 0 & 0 & 0 & 0 & 0 \\
\hline BA8 & 0 & 0 & 0 & 0 & 0 & 0 & 0 & 0 & 0 & 0 & 0 & 0 & 0 & 0 & 0 & 1 & 0 & 0 & 0 & 0 & 0 & 0 & 0 & 0 & 0 & 0 & 0 & 0 & 0 & 0 \\
\hline 49 & 0 & 0 & 0 & 0 & 0 & 0 & 0 & 0 & 0 & 0 & 0 & 1 & 0 & 0 & 1 & c & 0 & 0 & 0 & 0 & 0 & 0 & 0 & 0 & 0 & 0 & 0 & 0 & 0 & \\
\hline BA10 & 0 & 0 & 0 & 0 & 0 & 0 & 0 & 0 & 0 & 0 & 0 & 2 & $\underline{0}$ & 0 & 0 & $\underline{0}$ & 0 & 0 & 0 & 0 & 0 & 0 & 0 & 0 & 0 & 0 & 0 & $\underline{0}$ & 0 & \\
\hline A1 & 1 & 1 & 1 & 0 & 0 & 0 & 0 & 0 & 0 & 0 & 10 & 0 & 1 & 0 & 0 & 0 & 0 & 0 & 0 & 0 & 5 & 0 & 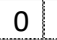 & 0 & 0 & 1 & & 0 & 0 & \\
\hline$A 2$ & 0 & 0 & 0 & 0 & 0 & 0 & 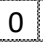 & 0 & 0 & 0 & 0 & 0 & 0 & 0 & 0 & 0 & 0 & 0 & 0 & 0 & 0 & 0 & 0 & 0 & 0 & 0 & & 0 & 0 & \\
\hline AA3 & 0 & 0 & 0 & 0 & 0 & 0 & 0 & 0 & 0 & 0 & 0 & 0 & 0 & 0 & 0 & 0 & 0 & 0 & 0 & 0 & 4 & 0 & 0 & 0 & 0 & 0 & & 0 & 0 & \\
\hline AA4 & 0 & 0 & 0 & 0 & 0 & 0 & 0 & 0 & 0 & 0 & 0 & 0 & 0 & 0 & 0 & 0 & 0 & 0 & 0 & 0 & 0 & 0 & 0 & 0 & 0 & 0 & 0 & 0 & 0 & \\
\hline AA5 & 0 & 0 & 17 & 0 & 0 & 0 & $c$ & 0 & 0 & 0 & 0 & 0 & 3 & 0 & 0 & 0 & 0 & 0 & 0 & 0 & 1 & 0 & 23 & 0 & 0 & 0 & 0 & 0 & 0 & \\
\hline AA6 & 0 & 0 & 0 & 0 & 0 & 0 & c & 0 & 0 & 0 & 0 & 0 & 0 & 0 & 0 & 0 & 0 & 0 & 0 & 0 & 0 & 0 & 0 & 5 & 0 & 0 & 0 & 0 & 0 & \\
\hline AA7 & 0 & 0 & 2 & 0 & 0 & 0 & 0 & 2 & 7 & 0 & 0 & 0 & 5 & 22 & 15 & 6 & 0 & 0 & 0 & 0 & 0 & 0 & 0 & 0 & 0 & 0 & 0 & 6 & 9 & \\
\hline $\mathrm{AA8}$ & 2 & 0 & 0 & 0 & 0 & 0 & 1 & 0 & 0 & 0 & 0 & 0 & 0 & 0 & 0 & 0 & 0 & 0 & 0 & 0 & 0 & 0 & 0 & 0 & 0 & 0 & 0 & 0 & 0 & \\
\hline AA9 & 1 & 0 & 0 & 0 & 0 & 0 & 0 & 0 & 0 & 0 & 0 & 0 & 0 & 0 & 0 & 0 & 1 & 0 & 0 & 0 & 0 & 0 & 1 & 0 & 0 & 0 & 0 & 0 & 0 & \\
\hline AA10 & 14 & 0 & 0 & 0 & 0 & 0 & 0 & 0 & 0 & 0 & 30 & 0 & 0 & 0 & 0 & 0 & 0 & 0 & 0 & 0 & 27 & 0 & 0 & 0 & 0 & 0 & 0 & 0 & 0 & 0 \\
\hline $\mathrm{BC} 11$ & 0 & 0 & 0 & 0 & 0 & 0 & 0 & 0 & 0 & 0 & 0 & 0 & 0 & 0 & 0 & 0 & 0 & 0 & 0 & 0 & 0 & 0 & 0 & 0 & 0 & 0 & 0 & 0 & 0 & 0 \\
\hline $\mathrm{BC} 12$ & 1 & 0 & 0 & 0 & 0 & 0 & 0 & 0 & 0 & 0 & 0 & 0 & 0 & 0 & 0 & 0 & 0 & 0 & 0 & 0 & 4 & 3 & 3 & 0 & 0 & 0 & 0 & 0 & 0 & 0 \\
\hline $\mathrm{BC} 13$ & 2 & 3 & 0 & 0 & 0 & 0 & 0 & 0 & 0 & 0 & 0 & 0 & 0 & 0 & 0 & 0 & 0 & 0 & 0 & 0 & 0 & 2 & 0 & 0 & 0 & 0 & 0 & 0 & 0 & \\
\hline $\mathrm{BC} 14$ & 1 & 3 & 0 & 0 & 0 & 0 & 0 & 0 & 0 & 0 & 0 & 0 & 0 & 0 & 0 & 0 & 0 & 0 & 0 & 0 & 1 & 0 & 0 & 1 & 0 & 0 & 1 & 0 & 0 & \\
\hline BC15 & 0 & 1 & 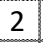 & 1 & 0 & 0 & 0 & 0 & 0 & 0 & 0 & 0 & 0 & 0 & 0 & 0 & 0 & 0 & 0 & 0 & 2 & 0 & 0 & 0 & 0 & 0 & 0 & 0 & 0 & \\
\hline $\mathrm{BC} 16$ & 1 & 1 & 1 & 0 & 0 & 0 & 0 & 0 & 0 & 0 & 0 & 0 & 0 & 0 & 0 & 0 & 0 & 0 & 0 & 0 & 0 & 1 & 0 & 0 & 1 & 0 & 0 & 0 & 0 & 0 \\
\hline $\mathrm{BC} 17$ & 1 & 0 & 0 & 0 & 0 & 0 & 0 & 0 & 0 & 0 & 0 & 0 & 0 & 0 & 1 & 1 & 0 & 0 & 0 & 0 & 0 & 0 & 0 & 0 & 0 & 0 & 0 & 0 & 0 & \\
\hline BC18 & 1 & 1 & 0 & 0 & 0 & 0 & 0 & 0 & 0 & 0 & 0 & 1 & 0 & 0 & 0 & 0 & 0 & 0 & 0 & 0 & 0 & 0 & 0 & 1 & 1 & 1 & 0 & 0 & 1 & \\
\hline BC19 & 0 & 0 & 1 & 0 & 1 & 0 & 0 & 1 & 0 & 0 & 0 & 0 & 0 & 0 & 0 & 0 & 0 & 0 & 0 & 0 & 0 & 0 & 2 & 1 & 0 & 0 & 0 & 1 & 0 & \\
\hline $\mathrm{BC} 20$ & $\underline{0}$ & 0 & 0 & 0 & 0 & 0 & 0 & 0 & 0 & 0 & 0 & 0 & 0 & 0 & 0 & 0 & 0 & 0 & 0 & 0 & 0 & 0 & 0 & 0 & 0 & 0 & 0 & $\underline{0}$ & 0 & $\underline{0}$ \\
\hline AC11 & 1 & 1 & 2 & 10 & 8 & 12 & 10 & 9 & 5 & 0 & 3 & 9 & 25 & 17 & 23 & 24. & 5 & 6 & 0 & 0 & 2 & 1 & 7 & 8 & 8 & 10. & 13 & 9 & 10 & \\
\hline AC12 & 6 & 0 & 2 & 6 & 4 & 9 & 4 & 7 & 3 & 0 & 2 & 0 & \begin{tabular}{|l|}
1 \\
\end{tabular} & 11 & 7 & 1 & 0 & 2 & 2 & 0 & 2 & 0 & 1 & 3 & 4 & 4 & 8 & 5 & 6 & \\
\hline AC13 & 1 & 3 & 2 & 1 & 4 & 8 & 9 & 4 & 2 & 0 & 2 & 3 & 3 & 4 & 1 & 3 & 1 & 0 & 0 & 0 & 2 & 2 & 0 & 1 & 3 & 3 & 4 & 1 & 3 & \\
\hline AC14 & 1 & 1 & 1 & 2 & 4 & 4 & 5 & 6 & 3 & 1 & 1 & 5 & 1 & 1 & 3 & 1 & 0 & 1 & 0 & 0 & 1 & \begin{tabular}{|l|} 
\\
\end{tabular} & 4 & 2 & 0 & 2 & 3 & 2 & 3 & \\
\hline AC15 & 4 & 2 & 0 & 2 & 8 & 2 & 7 & 0 & 0 & 0 & 3 & 2 & 5 & 2 & 9 & 8 & 5 & 1 & 0 & 0 & 1 & 2 & 2 & 5 & 5 & 1 & 2 & 1 & 0 & \\
\hline AC16 & 6 & 1 & 2 & 0 & 1 & 3 & 2 & 2 & 3 & 0 & 2 & 3 & 8 & 4 & 4 & 15 & 3 & 1 & 0 & 0 & 1 & 1 & 2 & 1 & 0 & 6 & 2 & 2 & 1 & \\
\hline AC17 & 0 & 5 & 2 & 8 & 4 & 2 & 10 & 5 & 4 & 2 & 1 & 0 & 6 & 1 & 10 & 5 & 10 & 1 & 0 & 0 & 1 & 2 & 3 & 3 & 0 & 1 & 10 & 6 & 4 & \\
\hline AC18 & 2 & 3 & 4 & 15 & 3 & 2 & 12 & 9 & 10 & 0 & 1 & 2 & 5 & 3 & 2 & 0 & 3 & 0 & 0 & 0 & 1 & 3 & 1 & 5 & 2 & 5 & \begin{tabular}{l|l}
5 & 1 \\
\end{tabular} & 11 & 2 & \\
\hline AC19 & 4 & 1 & 2 & 3 & 3 & 4 & 5 & 9 & 5 & v & 1 & 1 & 1 & 1 & 0 & 0 & 0 & 2 & 1 & 0 & 1 & $\begin{array}{ll} \\
\end{array}$ & 1 & 4 & 4 & 6 & 2 & 6 & 6 & \\
\hline $\mathrm{AC} 20$ & 2 & & & 4 & & & 2 & & 0 & & & 1 & & & 0 & & 0 & & & & & & & & & & & & & \\
\hline
\end{tabular}




\section{Table 3 Descriptive models parameters - baselines}

\section{Panel I : Trades and LOS w/ BPI}

\begin{tabular}{|c|c|c|c|c|c|c|c|c|c|c|c|c|c|c|c|c|}
\hline \multirow[b]{2}{*}{$m$} & \multicolumn{5}{|c|}{ BMW } & \multicolumn{6}{|c|}{ SAP } & \multicolumn{5}{|c|}{ ADS } \\
\hline & Buy* Sell* & Buy & \begin{tabular}{|l|} 
Sell \\
\end{tabular} & BA1* & AA1* & Buy* & Sell* & Buy & Sell & BA1* & AA1* & Buy* & Sell* & Buy & & BA1* $\mathrm{AA}$ \\
\hline$\widehat{\mu_{d}^{m}}$ & \begin{tabular}{|l|l|l|}
0.03 & 0.03
\end{tabular} & 0.02 & 0.02 & 0.04 & 0.05 & \begin{tabular}{|l|}
0.04 \\
\end{tabular} & 0.03 & 0.02 & 0.02 & 0.03 & 0.03 & 0.02 & 0.02 & 0.02 & 0.02 & 0.04 \\
\hline $\bar{d} / E\left[\lambda_{d}^{m}(t)\right]$ & \begin{tabular}{|l|l|}
0.56 & 0.55 \\
\end{tabular} & 0.63 & 0.61 & 0.32 & 0.36 & 0.67 & 0.67 & 0.61 & 0.61 & 0.29 & 0.28 & 0.56 & 0.57 & 0.79 & 0.80 & \begin{tabular}{|l|l|}
0.36 & 0.3 \\
\end{tabular} \\
\hline
\end{tabular}

\section{Panel II : LOS w/o BPI and LOC}

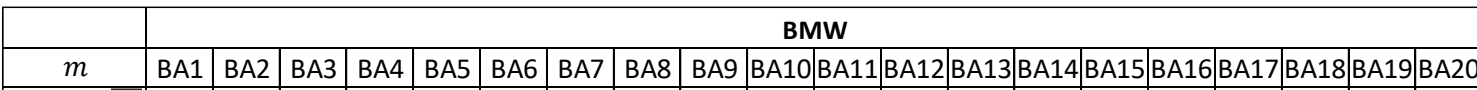

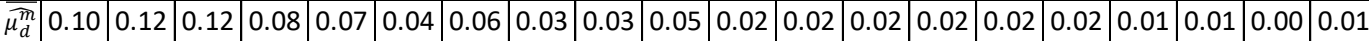

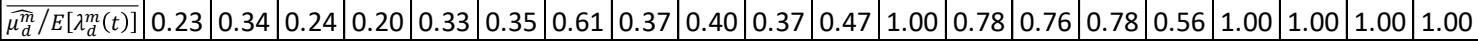

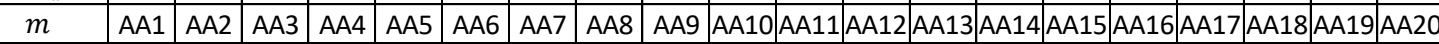

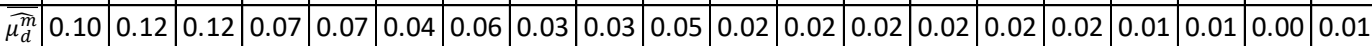
\begin{tabular}{|l|l|l|l|l|l|l|l|l|l|l|l|l|l|l|l|l|l|l|l|l|l}
$\overline{\mu_{d}^{m} / E\left[\lambda_{d}^{m}(t)\right]}$ & 0.22 & 0.32 & 0.24 & 0.18 & 0.35 & 0.36 & 0.65 & 0.44 & 0.41 & 0.37 & 0.50 & 0.76 & 1.00 & 0.77 & 0.79 & 0.84 & 1.00 & 1.00 & 1.00 & 1.00 \\
\hline
\end{tabular}

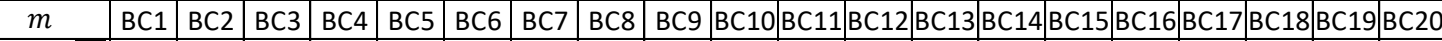
\begin{tabular}{l|l|l|l|l|l|l|l|l|l|l|l|l|l|l|l|l|l|l|l|l}
$\widehat{\mu_{d}^{m}}$ & 0.08 & 0.09 & 0.08 & 0.08 & 0.04 & 0.04 & 0.04 & 0.04 & 0.03 & 0.03 & 0.03 & 0.02 & 0.02 & 0.01 & 0.01 & 0.01 & 0.01 & 0.01 & 0.00 & 0.00
\end{tabular}

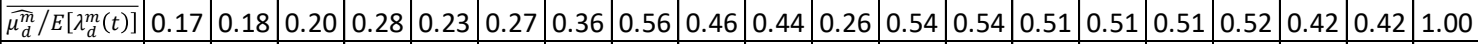

\begin{tabular}{l|l|l|l|l|l|l|l|l|l|l|l|l|l|l|l|l|l|l|l|l|l|l|l}
$m$ & AC1 & AC2 & AC3 & AC4 & AC5 & AC6 & AC7 & AC8 & AC9 & AC10 & AC11 & AC12 & AC13 & AC14 & AC15 & AC16 & AC17 & AC18 & AC19 & AC20 \\
\hline
\end{tabular}

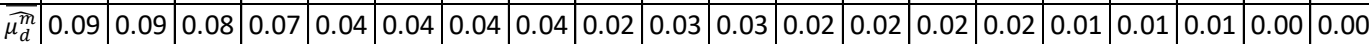
\begin{tabular}{|l|l|l|l|l|l|l|l|l|l|l|l|l|l|l|l|l|l|l|l|l|l|l|l|l}
$\overline{\mu_{d}^{m}} / E\left[\lambda_{d}^{m}(t)\right]$ & 0.19 & 0.19 & 0.21 & 0.24 & 0.23 & 0.26 & 0.37 & 0.58 & 0.38 & 0.45 & 0.26 & 0.54 & 0.53 & 0.70 & 0.72 & 0.50 & 0.43 & 0.44 & 0.42 & 1.00 \\
\hline
\end{tabular} SAP

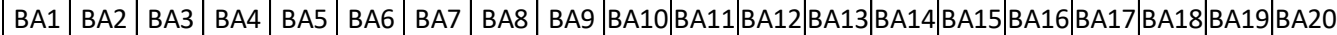

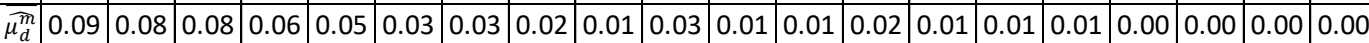

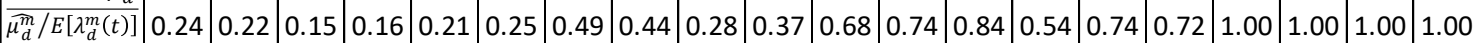

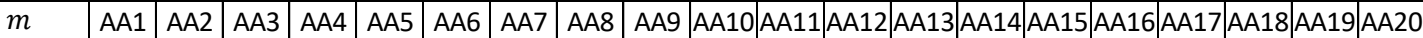

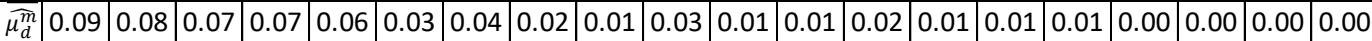

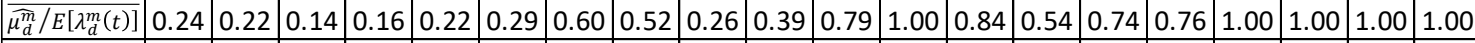

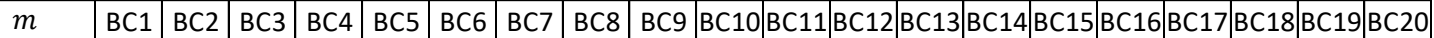

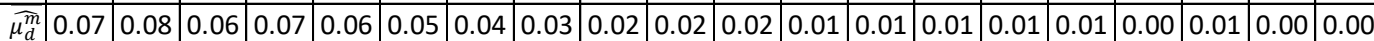

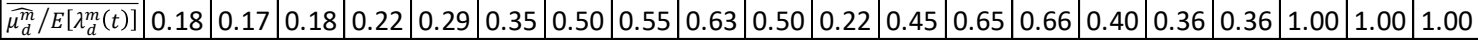

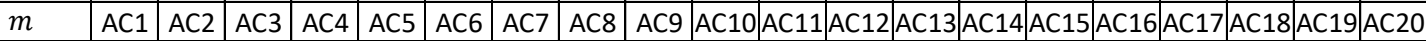

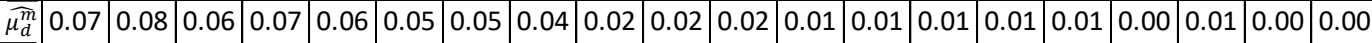

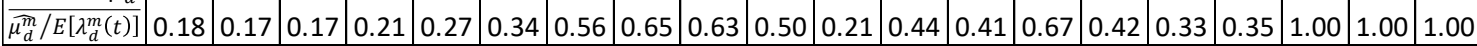

\begin{tabular}{|c|c|c|c|c|c|c|c|c|c|c|c|c|c|c|c|c|c|c|c|c|}
\hline & \multicolumn{20}{|c|}{ ADS } \\
\hline$m$ & BA1 & $\mathrm{A} 2$ & $\mathrm{~A} 3$ & $3 \mathrm{~A} 4$ & BA5 & BA6 & BA7 & BA8 & BA9 & B & BA11 & BA12 & BA13 & BA14 & BA15 & BA16 & 17 & $B$ & 9 & BA2 \\
\hline & 08 & 06 & 08 & 05 & 04 & 04 & 04 & 0.02 & 0.02 & 0.03 & 0.01 & 0.01 & 0.01 & 0.01 & 0.02 & 0.02 & 0.01 & .01 & .01 & 0.01 \\
\hline$/ E\left[\lambda_{d}^{m}(t)\right]$ & 32 & 33 & 30 & 22 & 31 & 32 & 52 & 33 & 35 & 37 & 48 & 75 & 75 & 73 & 0.75 & 0.78 & 00 & 00 & .00 & .0 \\
\hline$m$ & AA1 & AA2 & AA3 & AA4 & AA5 & AA6 & AA7 & AA8 & AA9 & AA10 & AA11 & AA12 & AA13 & AA14 & AA15 & AA16 & A17 & A18 & A19 & AA20 \\
\hline & 08 & 06 & 07 & 05 & 05 & 04 & 04 & 02 & 02 & 0.03 & 0.01 & 0.01 & 0.01 & 0.01 & 0.02 & 0.02 & 0.01 & 0.01 & .01 & 0.01 \\
\hline$/ E\left[\lambda_{d}^{m}(t)\right]$ & 0.33 & .32 & .27 & 0.20 & .34 & 0.32 & 0.44 & 0.35 & 0.35 & 0.37 & 0.52 & 1.00 & 0.75 & 0.74 & 0.76 & 0.79 & 1.00 & 1.00 & 1.00 & 1.00 \\
\hline$m$ & $\mathrm{BC} 1$ & $C 2$ & $\mathrm{BC} 3$ & BC4 & $\mathrm{BC5}$ & BC6 & $\mathrm{BC7}$ & $\mathrm{BC} 8$ & $\mathrm{BC} 9$ & $\mathrm{BC} 10$ & $\mathrm{BC} 11$ & $\mathrm{BC} 12$ & $\mathrm{BC} 13$ & $\mathrm{BC} 14$ & $\mathrm{BC} 15$ & $\mathrm{BC} 16$ & BC17 & BC18 & $\mathrm{BC} 1 \mathrm{~S}$ & $3 C 2$ \\
\hline & 05 & 06 & 06 & .06 & 05 & 05 & .03 & 04 & .03 & 0.02 & 0.02 & 0.02 & 01 & 0.01 & 0.01 & 0.01 & 0.01 & 0.01 & 0.01 & 00 \\
\hline$\frac{\widehat{m}}{d} / E\left[\lambda_{d}^{m}(t)\right]$ & 19 & 23 & 28 & 0.33 & 0.42 & 0.41 & 0.44 & 0.64 & 0.55 & 0.51 & 0.25 & 0.51 & 0.55 & 0.61 & 0.52 & 0.49 & 0.48 & 0.42 & 0.46 & 1.00 \\
\hline$m$ & AC1 & $A C 2$ & AC3 & AC4 & AC5 & AC6 & AC7 & AC8 & AC9 & AC10 & AC11 & AC12 & AC13 & AC14 & AC15 & AC16 & AC17 & AC18 & AC19 & $\mathrm{AC} 20$ \\
\hline & 0.05 & 0.07 & 0.06 & 0.06 & 05 & 05 & 0.03 & 0.04 & 0.02 & 0.02 & 0.02 & 0.01 & 0.01 & 0.01 & 0.01 & 0.01 & 0.01 & 0.01 & 0.01 & 0.00 \\
\hline$\frac{t_{d}^{m}}{d} / E\left[\lambda_{d}^{m}(t)\right]$ & 0.19 & .25 & 0.28 & 0.36 & 0.42 & 0.42 & 0.45 & 0.64 & 0.55 & 0.51 & 0.25 & 0.51 & 0.56 & 0.54 & 0.50 & 0.47 & 0.45 & 0.51 & 0.47 & 1.00 \\
\hline
\end{tabular}


Table 4 Descriptive models parameters - Hawkes events effects

Panel I: Buy*, Sell*, Buy, Sell, BA1* and AA1*

\begin{tabular}{|c|c|c|c|c|c|c|c|c|c|c|c|c|c|c|c|c|c|c|c|}
\hline \multirow{2}{*}{\multicolumn{2}{|c|}{$\bar{n}$}} & \multicolumn{6}{|c|}{ BMW } & \multicolumn{6}{|c|}{ SAP } & \multicolumn{6}{|c|}{ ADS } \\
\hline & & Buy* & Sell* & Buy & Sell & BA1* & AA1* & Buy* & Sell* & Buy & Sell & BA1* & AA1* & Buy* & Sell* & Buy & Sell & BA1* & AA1* \\
\hline Buy* & $\frac{\overline{\hat{\alpha}_{n / m}}}{\hat{\beta}_{n / m}}$ & & & & & $\begin{array}{c}27 \\
101 \\
\end{array}$ & $\begin{array}{l}5.5 \\
29 \\
\end{array}$ & & & & & $\begin{array}{c}31 \\
118 \\
\end{array}$ & $\begin{array}{l}12 \\
86 \\
\end{array}$ & & & & & $\begin{array}{l}26 \\
90 \\
\end{array}$ & $\begin{array}{l}12 \\
92\end{array}$ \\
\hline Sell* & $\overline{\frac{\hat{\alpha}_{n / m}}{\hat{\beta}_{n / m}}}$ & & & & & $\begin{array}{c}8.9 \\
71 \\
\end{array}$ & $\begin{array}{c}28 \\
103 \\
\end{array}$ & & & & & $\begin{array}{l}11 \\
76\end{array}$ & \begin{tabular}{|c|}
35 \\
123 \\
\end{tabular} & & & & & $\begin{array}{l}13 \\
88\end{array}$ & $\begin{array}{l}27 \\
89\end{array}$ \\
\hline Buy & $\frac{\hat{\alpha}_{n / m}}{\hat{\beta}_{n / m}}$ & $\begin{array}{c}38 \\
162 \\
\end{array}$ & & $\begin{array}{c}8.5 \\
45 \\
\end{array}$ & & $\begin{array}{l}10 \\
87 \\
\end{array}$ & & \begin{tabular}{|c|}
19 \\
107 \\
\end{tabular} & & $\begin{array}{c}8.8 \\
41 \\
\end{array}$ & & $\begin{array}{c}11 \\
108 \\
\end{array}$ & & \begin{tabular}{|c|}
34 \\
169 \\
\end{tabular} & & $\begin{array}{l}4.9 \\
27 \\
\end{array}$ & & $\begin{array}{c}14 \\
113 \\
\end{array}$ & \\
\hline Sell & $\overline{\frac{\hat{\alpha}_{n / m}}{\hat{\beta}_{n / m}}}$ & & $\begin{array}{c}36 \\
154 \\
\end{array}$ & & $\begin{array}{l}9.0 \\
42 \\
\end{array}$ & & $\begin{array}{l}11 \\
86\end{array}$ & & $\begin{array}{c}20 \\
113 \\
\end{array}$ & & $\begin{array}{l}7.8 \\
37 \\
\end{array}$ & & $\begin{array}{c}11 \\
106 \\
\end{array}$ & & \begin{tabular}{|c|}
33 \\
164 \\
\end{tabular} & & $\begin{array}{l}4.1 \\
23 \\
\end{array}$ & & $\begin{array}{c}14 \\
101 \\
\end{array}$ \\
\hline BA1* & $\begin{array}{l}\frac{\hat{\alpha}_{n / m}}{\hat{\beta}_{n / m}} \\
\end{array}$ & $\begin{array}{c}3.5 \\
63 \\
\end{array}$ & $\begin{array}{c}17 \\
218 \\
\end{array}$ & & $\begin{array}{l}1.6 \\
47 \\
\end{array}$ & $\begin{array}{c}15 \\
341 \\
\end{array}$ & & & \begin{tabular}{|c|}
14 \\
144 \\
\end{tabular} & & $\begin{array}{l}1.9 \\
38\end{array}$ & & & $\begin{array}{l}3.7 \\
69\end{array}$ & \begin{tabular}{|c|}
9.1 \\
155 \\
\end{tabular} & & & $\begin{array}{c}20 \\
348\end{array}$ & \\
\hline AA1* & $\frac{\overline{\hat{\alpha}_{n / m}}}{\hat{\beta}_{n / m}}$ & \begin{tabular}{|c|}
17 \\
215 \\
\end{tabular} & $\begin{array}{c}3.4 \\
61 \\
\end{array}$ & $\begin{array}{l}1.5 \\
44\end{array}$ & & & $\begin{array}{c}15 \\
298 \\
\end{array}$ & \begin{tabular}{|c|}
11 \\
108 \\
\end{tabular} & & $\begin{array}{l}1.9 \\
33 \\
\end{array}$ & & & & \begin{tabular}{|l|}
8.5 \\
137 \\
\end{tabular} & $\begin{array}{l}3.6 \\
62 \\
\end{array}$ & & & & $\begin{array}{c}19 \\
337 \\
\end{array}$ \\
\hline BA1 & $\frac{\overline{\hat{\alpha}_{n / m}}}{\hat{\beta}_{n / m}}$ & & & & & $\begin{array}{c}11 \\
232 \\
\end{array}$ & & & & & & $\begin{array}{c}12 \\
269 \\
\end{array}$ & & & & & & $\begin{array}{l}8.5 \\
198 \\
\end{array}$ & \\
\hline AA1 & $\frac{\overline{\hat{\alpha}_{n / m}}}{\hat{\beta}_{n / m}}$ & & & & & & $\begin{array}{l}8.2 \\
147\end{array}$ & & & & & & $\begin{array}{c}12 \\
268\end{array}$ & & & & & & \begin{tabular}{|c|}
8.5 \\
188 \\
\end{tabular} \\
\hline $\mathrm{BC} 1$ & $\frac{\hat{\hat{\alpha}}_{n / m}}{\hat{\beta}_{n / m}}$ & & & & & $\begin{array}{l}2.0 \\
74 \\
\end{array}$ & $\begin{array}{c}14 \\
309 \\
\end{array}$ & & & & & $\begin{array}{l}0.6 \\
16 \\
\end{array}$ & $\begin{array}{c}10 \\
283 \\
\end{array}$ & & & & & $\begin{array}{l}4.7 \\
93 \\
\end{array}$ & $\begin{array}{c}12 \\
237 \\
\end{array}$ \\
\hline AC1 & $\frac{\overline{\hat{\alpha}_{n / m}}}{\hat{\beta}_{n / m}}$ & & & & & $\begin{array}{c}14 \\
327 \\
\end{array}$ & & & & & & $\begin{array}{c}11 \\
299 \\
\end{array}$ & $\begin{array}{l}0.8 \\
24 \\
\end{array}$ & & & & & $\begin{array}{c}12 \\
233 \\
\end{array}$ & $\begin{array}{l}5.1 \\
113 \\
\end{array}$ \\
\hline
\end{tabular}

For each Hawkes effect $n / m$, this figure presents estimated parameters $\hat{\alpha}_{d}^{n / m}$ and $\hat{\beta}_{d}^{n / m}$ daily average. 
Panel II: BA1 to BA10

\begin{tabular}{|c|c|c|c|c|c|c|c|c|c|c|c|c|c|c|c|c|c|c|c|c|c|c|c|c|c|c|c|c|c|c|c|}
\hline \multirow{2}{*}{\multicolumn{2}{|c|}{$n \quad m$}} & \multicolumn{10}{|c|}{ BMW } & \multicolumn{10}{|c|}{ SAP } & \multicolumn{10}{|c|}{ ADS } \\
\hline & & BA1 & BA2 & $\mathrm{BA} 3$ & BA4 & BA5 & BA6 & BA7 & BA8 & BA9 & BA10 & BA1 & BA2 & BA3 & BA4 & BA5 & BA6 & BA7 & BA8 & BA9 & BA10 & BA1 & 1 BA2 & \begin{tabular}{|l|l|}
2 & $B A 3$ \\
\end{tabular} & \begin{tabular}{|l|l|}
3 & BA4 \\
\end{tabular} & \begin{tabular}{|l|l|}
4 & BA5 \\
\end{tabular} & BA6 & BA7 & BA8 & BA9 & BA10 \\
\hline Buy* & $\sqrt{\hat{\hat{\alpha}}_{n / m}}$ & 95 & 78 & 56 & 35 & 17 & & & & & & 89 & 69 & 46 & 21 & \begin{tabular}{|l|}
9.5 \\
\end{tabular} & & & & & & 68 & \begin{tabular}{|l|l|}
8 & 69 \\
\end{tabular} & \begin{tabular}{|l|l|}
9 & 55 \\
\end{tabular} & \begin{tabular}{|l|l|}
5 & 43 \\
\end{tabular} & \begin{tabular}{|l|l|}
3 & 21 \\
\end{tabular} & & & & & \\
\hline & $\hat{\beta}_{n / m}$ & 212 & 233 & 183 & 158 & 94 & & & & & & 201 & 194 & 146 & 137 & 95 & & & & & & 170 & 200 & $\begin{array}{l}0 \quad 148 \\
\end{array}$ & $\begin{array}{lll}8 & 182 \\
\end{array}$ & \begin{tabular}{ll|}
2 & 115 \\
\end{tabular} & & & & & \\
\hline Sell* & $\widehat{\widehat{\hat{\alpha}_{n / m}}}$ & 111 & 6.7 & 89 & 11 & 11 & 5.7 & & 7.2 & 4.6 & 88 & 129 & 10 & 103 & 12 & 14 & 7.5 & 5.6 & 7.6 & 10 & 68 & 65 & 5.8 & \begin{tabular}{|l|l|}
8 & 80
\end{tabular} & \begin{tabular}{|l|l|}
0 & 11 \\
\end{tabular} & \begin{tabular}{|l|l|}
1 & 15 \\
\end{tabular} & & & 8.8 & 10 & 83 \\
\hline & $\hat{\beta}_{n / m}$ & 348 & 132 & 313 & 69 & 63 & 26 & & 29 & 23 & 333 & 328 & 190 & 263 & 52 & 81 & 57 & 33 & 39 & 109 & 242 & 268 & 8132 & 2286 & $6 \quad 88$ & \begin{tabular}{ll|}
8 & 67 \\
\end{tabular} & & & 68 & 63 & 281 \\
\hline Buy & $\overline{\hat{\alpha}_{n / m}}$ & 45 & 60 & 32 & & & & & & & & 28 & 37 & 14 & & & & & & & & 54 & \begin{tabular}{|l|l|}
4 & 57 \\
\end{tabular} & \begin{tabular}{|l|l|}
7 & 29 \\
\end{tabular} & & & & & & & \\
\hline & $\hat{\hat{\beta}}_{n / m}$ & 207 & 475 & 369 & & & & & & & & 184 & 401 & 240 & & & & & & & & 273 & $\begin{array}{l}3434 \\
\end{array}$ & $\begin{array}{ll}4 & 293 \\
\end{array}$ & & & & & & & \\
\hline Sell & $\sqrt{\frac{\hat{\hat{\alpha}}_{n / m}}{\hat{\beta}_{n / m}}}$ & & & & & & & & & & & $\begin{array}{r}3.8 \\
60\end{array}$ & & & & & & & & & & & & & & & & & & & \\
\hline BA1* & $* \widehat{\hat{\alpha}}_{n / m}$ & 39 & 65 & 101 & 41 & 21 & \begin{tabular}{|l|}
9.3 \\
\end{tabular} & & & & & 19 & 48 & 115 & 17 & \begin{tabular}{|l|}
4.7 \\
\end{tabular} & 2.8 & 4.2 & & & & 28 & \begin{tabular}{|l|l|}
8 & 40 \\
\end{tabular} & \begin{tabular}{|l|l|}
0 & 97 \\
\end{tabular} & \begin{tabular}{|l|l|}
7 & 43 \\
\end{tabular} & \begin{tabular}{|l|l|}
3 & 22 \\
\end{tabular} & \begin{tabular}{|l|}
9.0 \\
\end{tabular} & \begin{tabular}{|l|}
5.9 \\
\end{tabular} & & & \\
\hline & $\hat{\beta}_{n / m}$ & 269 & 393 & 322 & 179 & 130 & 98 & & & & & 197 & 287 & 231 & 76 & 39 & 36 & 76 & & & & 217 & 7345 & 5320 & $\begin{array}{ll}0 & 195 \\
\end{array}$ & \begin{tabular}{lll|}
5 & 185 \\
\end{tabular} & 80 & 71 & & & \\
\hline AA1* & $\sqrt{\frac{\hat{\alpha}_{n / m}}{\hat{\beta}_{n / m}}}$ & & & $\begin{array}{r}10 \\
316 \\
\end{array}$ & & & & & & & & & & & & & & & & $\begin{array}{r}4.5 \\
171 \\
\end{array}$ & & & & & & & & & & & \\
\hline BA1 & $\hat{\hat{\alpha}}_{n / m}$ & 26 & 34 & 52 & 19 & & & & & & & 16 & 39 & 24 & & & & & & & & 16 & \begin{tabular}{|l|l|}
5 & 22 \\
\end{tabular} & \begin{tabular}{|l|l|}
2 & 24 \\
\end{tabular} & \begin{tabular}{|l|l|}
4 & 11 \\
\end{tabular} & & & & & & \\
\hline & $\hat{\beta}_{n / m}$ & 353 & 604 & 770 & 506 & & & & & & & 240 & 593 & 463 & & & & & & & & 247 & 486 & $6 \quad 434$ & $4 \quad 426$ & & & & & & \\
\hline BA2 & $\hat{\hat{\alpha}}_{n / m}$ & 3.0 & 6.0 & 8.6 & & & & & & & & 2.5 & & 13 & & & & & & & & & 9.0 & $\begin{array}{l}0.9 \\
\end{array}$ & & & & & & & \\
\hline & $\hat{\beta}_{n / m}$ & 53 & 106 & 136 & & & & & & & & 64 & & 262 & & & & & & & & & 216 & $\begin{array}{ll}6 & 124\end{array}$ & & & & & & & \\
\hline BA3 & $\overline{\hat{\alpha}_{n / m}}$ & & 5.5 & 7.9 & 2.2 & & & & & & & & 13 & 14 & 6.4 & & & & & & & & 8.1 & \begin{tabular}{|l|l|}
1 & 8.2
\end{tabular} & \begin{tabular}{|l|l|}
2 & 3.5 \\
\end{tabular} & & & & & & \\
\hline & $\hat{\beta}_{n / m}$ & & 105 & 107 & 50 & & & & & & & & 308 & 209 & 163 & & & & & & & & 266 & $6 \quad 177$ & \begin{tabular}{l|l|}
7 & 99 \\
\end{tabular} & & & & & & \\
\hline BA4 & $\overline{\hat{\alpha}_{n / m}}$ & & & & & 3.7 & & & & & & & & & & 2.8 & & & & & & & & & & 2.3 & & & & & \\
\hline & $\hat{\beta}_{n / m}$ & & & & & 71 & & & & & & & & & & 91 & & & & & & & & & & 70 & & & & & \\
\hline BA5 & $\overline{\hat{\alpha}_{n / m}}$ & & & & 9.3 & & 3.6 & & & & & & & & & & & & & & & & & & & & 3.4 & & & & \\
\hline & $\hat{\beta}_{n / m}$ & & & & 221 & & 58 & & & & & & & & & & & & & & & & & & & & 62 & & & & \\
\hline BA6 & $\overline{\hat{\alpha}_{n / m}}$ & & & & & & & 3.3 & & & & & & & & & & 1.5 & & & & & & & & 13 & & 2.3 & & & \\
\hline & $\hat{\hat{\beta}}_{n / m}$ & & & & & & & 29 & & & & & & & & & & 39 & & & & & & & & 344 & & 30 & & & \\
\hline BA7 & $\overline{\hat{\alpha}}_{n / m}$ & & & & & & & & 3.3 & & & & & & & & & & 1.9 & & & & & & & & & & 3.0 & & \\
\hline & $\hat{\beta}_{n / m}$ & & & & & & & & 34 & & & & & & & & & & 30 & & & & & & & & & & 49 & & \\
\hline BA8 & $\overline{\frac{\hat{\alpha}_{n / m}}{\hat{\beta}_{n / m}}}$ & & & & & & & & & $\begin{array}{r}5.8 \\
41\end{array}$ & & & & & & & & & & $\begin{array}{r}3.4 \\
109 \\
\end{array}$ & & & & & & & & & & \begin{tabular}{|r|}
5.0 \\
42 \\
\end{tabular} & \\
\hline BA9 & $\hat{\hat{\alpha}}_{n / m}$ & & & & & & & & 6.6 & & 18 & & & & & & & & & & 12 & & & & & & & & & & 16 \\
\hline & $\hat{\beta}_{n / m}$ & & & & & & & & 109 & & 56 & & & & & & & & & & 49 & & & & & & & & & & 59 \\
\hline AA3 & $\overline{\hat{\alpha}}_{n / m}$ & & & 2.6 & & & & & & & & & & & & & & & & & & & & & & & & & & & \\
\hline & $\hat{\beta}_{n / m}$ & & & 83 & & & & & & & & & & & & & & & & & & & & & & & & & & & \\
\hline BC1 & $\overline{\hat{\alpha}_{n / m}}$ & 61 & 24 & 34 & & & & & & & 28 & 44 & 15 & 33 & 9.5 & & 3.3 & & & & 24 & 46 & \begin{tabular}{|l|} 
\\
\end{tabular} & \begin{tabular}{|l|l|}
3 & 39 \\
\end{tabular} & \begin{tabular}{|l|l|}
9 & 6.6 \\
\end{tabular} & & & & \begin{tabular}{|l|}
8.2 \\
\end{tabular} & & 35 \\
\hline & $\hat{\beta}_{n / m}$ & 626 & 769 & 531 & & & & & & & 572 & 496 & 564 & 461 & 341 & & 117 & & & & 560 & 442 & 555 & 517 & 7249 & & & & 244 & & 538 \\
\hline $\mathrm{BC2}$ & $\overline{\hat{\alpha}_{n / m}}$ & 1.4 & 12 & 61 & 2.2 & & & & & & & 0.8 & 2.2 & 39 & 13 & & & & & 19 & & 2.9 & 13 & \begin{tabular}{|l|l|}
8 & 54 \\
\end{tabular} & \begin{tabular}{|l|l|}
4 & 2.0
\end{tabular} & & & & 2.7 & 17 & \\
\hline & $\overline{\hat{\beta}_{n / m}}$ & 50 & 516 & 975 & 57 & & & & & & & 17 & 50 & 825 & 370 & & & & & 681 & & 63 & 447 & \begin{tabular}{l|l|}
7 & 884 \\
\end{tabular} & $\begin{array}{l}4 \\
4\end{array}$ & & & & 75 & 362 & \\
\hline BC3 & $\overline{\hat{\alpha}}_{n / m}$ & & 31 & 5.7 & 45 & & & & & & 39 & & 14 & 0.9 & 10 & 8.1 & & & & & & & 20 & & 14 & & & & & & 31 \\
\hline & $\hat{\beta}_{n / m}$ & & 865 & 135 & 582 & & & & & & 840 & & 514 & 6.2 & 165 & 177 & & & & & & & 592 & & 139 & & & & & & 597 \\
\hline BC4 & $\overline{\hat{\alpha}_{n / m}}$ & & & & 8.5 & 11 & & & & & & & & & 0.5 & 4.1 & 2.7 & & & & & & & & & 13 & & & & & \\
\hline & $\hat{\hat{\beta}}_{n / m}$ & & & & 229 & 174 & & & & & & & & & 1.6 & 96 & 77 & & & & & & & & & 235 & & & & & \\
\hline BC5 & $\widehat{\hat{\alpha}}_{n / m}$ & & & & 67 & 4.0 & & & & & & & & & & 0.8 & & & & & & & & & 68 & \begin{tabular}{|l|l|}
8 & 2.8
\end{tabular} & 4.9 & & & & \\
\hline & $\hat{\beta}_{n / m}$ & & & & 897 & 40 & & & & & & & & & & 2.2 & & & & & & & & & 875 & $5 \quad 35$ & 76 & & & & \\
\hline BC6 & $\widehat{\hat{\alpha}}_{n / m}$ & & & & 4.0 & & 2.8 & & & & & & & & 2.4 & & 1.4 & & & & & & & & 3.0 & & 1.3 & & & & \\
\hline & $\hat{\beta}_{n / m}$ & & & & 67 & & 36 & & & & & & & & 24 & & 6.7 & & & & & & & & 16 & & 11 & & & & \\
\hline BC7 & $\widehat{\hat{\alpha}_{n / m}}$ & & & & & & & 4.1 & & & & & & & & & & 1.3 & & & & & & & & & & 2.3 & & & \\
\hline & $\hat{\hat{\beta}}_{n / m}$ & & & & & & & 38 & & & & & & & & & & 18 & & & & & & & & & & 19 & & & \\
\hline BC8 & $\hat{\hat{\alpha}}_{n / m}$ & & & & & & & & 3.3 & & & & & & & & & & 1.4 & & & & & & & & & & 2.7 & & \\
\hline & $\hat{\beta}_{n / m}$ & & & & & & & & 45 & & & & & & & & & & 33 & & & & & & & & & & 46 & & \\
\hline AC1 & $\overline{\hat{\alpha}_{n / m}}$ & 42 & 31 & 41 & 24 & & & & & & & 30 & 27 & 28 & 8.1 & & & & & & & 27 & 16 & \begin{tabular}{|l|l|}
6 & 26 \\
\end{tabular} & \begin{tabular}{|l|l|}
6 & 19 \\
\end{tabular} & 7.1 & & & & & \\
\hline & $\overline{\hat{\beta}_{n / m}}$ & 458 & 552 & 561 & 491 & & & & & & & 356 & 401 & 427 & 248 & & & & & & & 283 & 328 & \begin{tabular}{|l|l|}
833 \\
\end{tabular} & 375 & 166 & & & & & \\
\hline AC2 & $\hat{\hat{\alpha}}_{n / m}$ & 5.2 & 11 & 22 & & & & & & & & 2.6 & 6.2 & 10 & & & & & & & & 3.2 & 6.9 & \begin{tabular}{|l|l|}
9 & 12 \\
\end{tabular} & 26.9 & & & & & & \\
\hline & $\hat{\hat{\beta}}_{n / m}$ & 127 & 313 & 507 & & & & & & & & 63 & 131 & 211 & & & & & & & & 74 & 200 & 264 & 4234 & & & & & & \\
\hline AC3 & $\overline{\hat{\alpha}}_{n / m}$ & 4.1 & 11 & 18 | 18 | & 7.5 & & & & & & & & 2.9 & 3.3 & & & & & & & & 1.8 & 2.8 & \begin{tabular}{|l|l|}
8 & 10
\end{tabular} & \begin{tabular}{|l}
5.3 \\
\end{tabular} & & & & & & \\
\hline & $\hat{\hat{\beta}}_{n / m}$ & 89 & 220 & 205 & 196 & & & & & & & & 64 & 57 & & & & & & & & 43 & 81 & 182 & 2157 & & & & & & \\
\hline AC4 & \begin{tabular}{|l}
$\frac{\hat{\alpha}_{n / m}}{\hat{\beta}_{n / m}}$ \\
\end{tabular} & & & & & & & & & & & & & & & & & & & & & & & & $\begin{array}{r}3.6 \\
123 \\
\end{array}$ & & & & & & \\
\hline
\end{tabular}




\section{Panel III: AA1 to AA10}

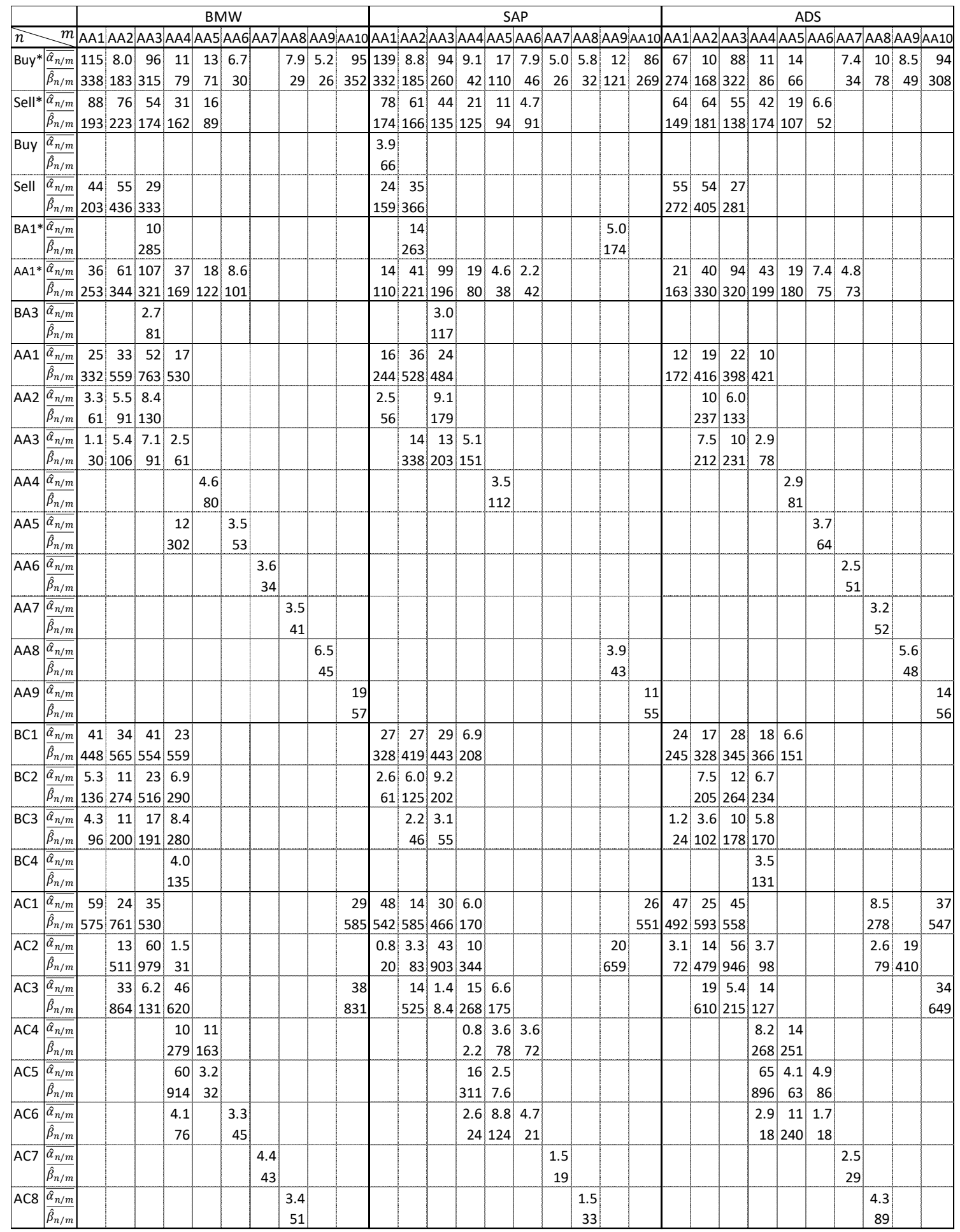


Panel IV: BC1 to BC10

\begin{tabular}{|c|c|c|c|c|c|c|c|c|c|c|c|c|c|c|c|c|c|c|c|c|c|c|c|c|c|c|c|c|c|c|c|}
\hline \multirow{2}{*}{\multicolumn{2}{|c|}{$n \quad m$}} & \multicolumn{10}{|c|}{ BMW } & \multicolumn{10}{|c|}{ SAP } & \multicolumn{10}{|c|}{ ADS } \\
\hline & & $\mathrm{BC} 1$ & $\mathrm{BC2}$ & $\mathrm{BC3}$ & $\mathrm{BC4}$ & $\mathrm{BC5}$ & $\mathrm{BC6}$ & $B C 7$ & $7 \mathrm{BC} 8$ & $B C 9$ & \begin{tabular}{|l|l|}
$9 B C 10$ \\
\end{tabular} & $B C 1$ & BC2 & BC3 & $\mathrm{BC} 4$ & $\mathrm{BC5}$ & $\mathrm{BC6}$ & BC7 & $\mathrm{BC} 8$ & BC9 & $\mathrm{BC} 10$ & $\mathrm{BC} 1$ & $\mathrm{BC2}$ & $2 \mathrm{BC3}$ & BC4 & $4 \mathrm{BC5}$ & $\mathrm{BC6}$ & BC7 & $B C 8$ & $\mathrm{BC} 9$ & $\mathrm{BC} 10$ \\
\hline Buy* & $\widehat{\hat{\alpha}}_{n / m}$ & & 10 & 18 & 24 & 14 & 7.5 & & & 4.2 & \begin{tabular}{|l|l|}
2 & 5.0
\end{tabular} & & & 16 & 21 & 13 & 5.0 & 6.1 & & & 8.2 & & 10 & \begin{tabular}{|l|l|} 
& 10
\end{tabular} & 16 & \begin{tabular}{|l|l|}
5 & 10 \\
\end{tabular} & 8.1 & & & 4.8 & 6.0 \\
\hline & $\hat{\beta}_{n / m}$ & & 94 & 145 & 169 & 105 & 77 & & & 33 & $\begin{array}{l}3 \quad 34 \\
\end{array}$ & & & 164 & 144 & 88 & 36 & 47 & & & 57 & & 70 & 64 & 110 & 68 & 76 & & & 38 & 39 \\
\hline Sell* & $\overline{\hat{\alpha}}_{n / m}$ & 119 & 167 & 78 & 39 & 24 & 19 & 14 & \begin{tabular}{|l|l}
4 & 8.2
\end{tabular} & 5.1 & & 108 & 181 & 60 & 36 & 22 & 16 & 10 & 2.8 & & & 113 & 151 & 169 & 33 & \begin{tabular}{|l|}
3 \\
3
\end{tabular} & 17 & 7) 15 & 8.2 & 5.6 & \\
\hline & $\hat{\beta}_{n / m}$ & 149 & 199 & 142 & 158 & 124 & 185 & 114 & \begin{tabular}{l|l}
4 & 78 \\
\end{tabular} & 81 & & 125 & 193 & 152 & 164 & 132 & 112 & 110 & 61 & & & 153 & 185 & 143 & 123 & 3102 & 116 & 161 & 71 & 85 & \\
\hline Buy & $\hat{\bar{\alpha}}_{n / m}$ & & & & & & & & & & & & & & & & & & & & & & & & & & & & & & \\
\hline & $\hat{\beta}_{n / m}$ & & & & & & & & & & & & & & & & & & & & & & & & & & & & & & \\
\hline Sell & $\underline{\hat{\alpha}_{n / m}}$ & 16 & 63 & 51 & 32 & 15 & 9.3 & 9.2 & 211 & & & 13 & 63 & 36 & 17 & 8.9 & & & & & & 25 & 54 & $\begin{array}{l}4 \\
4\end{array}$ & 18 & & 7.2 & & & & \\
\hline & $\hat{\beta}_{n / m}$ & 84 & 403 & 330 & 246 & 183 & 138 & 134 & 167 & & & 87 & 357 & 289 & 239 & 150 & & & & & & 145 & 364 & 4291 & 206 & & 88 & & & & \\
\hline BA1* & $\overline{\hat{\alpha}}_{n / m}$ & & 57 & 30 & 128 & 40 & 10 & 12 & \begin{tabular}{|l|l|}
2 & 10
\end{tabular} & 15 & 39 & & 61 & 24 & 138 & 21 & 8.1 & 6.5 & 7.7 & 6.5 & 18 & & 15 & $5 \quad 20$ & 103 & \begin{tabular}{|l|l|}
3 & 24 \\
\end{tabular} & \begin{tabular}{|l|}
8.3 \\
\end{tabular} & \begin{tabular}{|l|} 
\\
\end{tabular} & 6.9 & 10 & 27 \\
\hline & $\hat{\beta}_{n / m}$ & & 250 & 298 & 496 & 327 & 27 & 43 & \begin{tabular}{|l|l}
3 & 56 \\
\end{tabular} & 115 & $5 \quad 236$ & & 255 & 250 & 444 & 142 & 24 & 28 & 55 & 68 & 207 & & 87 & $7 \quad 187$ & 450 & 258 & 37 & $7 \quad 48$ & 50 & 93 & 187 \\
\hline AA1* & $\hat{\hat{\alpha}}_{n / m}$ & 121 & 63 & 53 & 25 & 11 & 5.6 & & & & & 116 & 59 & 28 & 11 & 6.3 & & & & & & 72 & 45 & 54 & 20 & 8.4 & 5.9 & 3.6 & & & \\
\hline & $\hat{\beta}_{n / m}$ & 421 & 360 & 356 & 242 & 156 & 145 & & & & & 383 & 262 & 223 & 149 & 107 & & & & & & 270 & 243 & 3277 & 202 & 113 & \begin{tabular}{l|l|}
3 & 98 \\
\end{tabular} & \begin{tabular}{|l|l|}
3 & 91 \\
\end{tabular} & & & \\
\hline BA1 & $\underline{\hat{\alpha}_{n / m}}$ & 5.2 & 26 & & 18 & & & & & & & 3.4 & 16 & \begin{tabular}{|l|}
19 \\
\end{tabular} & & & & & & & & 4.8 & 10 & & & & & & & & \\
\hline & $\hat{\beta}_{n / m}$ & 53 & 891 & & 648 & & & & & & & 18 & 619 & 708 & & & & & & & & 66 & 324 & & & & & & & & \\
\hline BA2 & $\hat{\hat{\alpha}}_{n / m}$ & & 6.8 & & & & & & & & & & 1.1 & & & & & & & & & & & & & & & & & & \\
\hline & $\hat{\beta}_{n / m}$ & & 223 & & & & & & & & & & 7.6 & & & & & & & & & & & & & & & & & & \\
\hline BA3 & $\frac{\hat{\alpha}_{n / m}}{\hat{\hat{n}_{1}}}$ & & & 2.6 & & & & & & & & & & 0.8 & & & & & & & & & & & & & & & & & \\
\hline & $\hat{\beta}_{n / m}$ & & & 81 & & & & & & & & & & 5.8 & & & & & & & & & & & & & & & & & \\
\hline BA4 & $\hat{\hat{\alpha}}_{n / m}$ & & & & & & & & & & & & & & 2.5 & & & & & & & & & & & & & & & & \\
\hline & $\hat{\beta}_{n / m}$ & & & & & & & & & & & & & & 46 & & & & & & & & & & & & & & & & \\
\hline BA5 & $\hat{\alpha}_{n / m}$ & & & & & 0.3 & & & & & & & & & & 3.2 & & & & & & & & & & & & & & & \\
\hline & $\hat{\beta}_{n / m}$ & & & & & 2.9 & & & & & & & & & & 32 & & & & & & & & & & & & & & & \\
\hline BA6 & $\overline{\hat{\alpha}}_{n / m}$ & & & & & & & & & & & & & & & & 0.6 & & & & & & & & & & & & & & \\
\hline & $\hat{\beta}_{n / m}$ & & & & & & & & & & & & & & & & 2.7 & & & & & & & & & & & & & & \\
\hline AA1 & $\underline{\overline{\hat{\alpha}}_{n / m}}$ & 24 & 36 & 34 & & & & & & & & 19 & 24 & 12 & & & & & & & & 18 & 16 & \begin{tabular}{|l|}
3 \\
\end{tabular} & & & & & & & \\
\hline & $\hat{\beta}_{n / m}$ & 392 & 682 & 687 & & & & & & & & 289 & 414 & 370 & & & & & & & & 271 & 333 & 320 & & & & & & & \\
\hline AA2 & $\hat{\hat{\alpha}}_{n / m}$ & 7.1 & 6.7 & 7.2 & & & & & & & & 4.5 & 3.0 & & & & & & & & & 6.1 & 3.7 & 3.7 & & & & & & & \\
\hline & $\hat{\beta}_{n / m}$ & 205 & 199 & 235 & & & & & & & & 115 & 65 & & & & & & & & & 154 & 111 & 101 & & & & & & & \\
\hline $\mathrm{AA} 3$ & $\underline{\hat{\alpha}_{n / m}}$ & 1.4 & 2.2 & 3.2 & & & & & & & & & \begin{tabular}{|l|}
1.4 \\
\end{tabular} & & & & & & & & & 2.3 & 3.5 & 3.8 & & & & & & & \\
\hline & $\hat{\beta}_{n / m}$ & 39 & 65 & 91 & & & & & & & & & 26 & & & & & & & & & 64 & 96 & 96 & & & & & & & \\
\hline $\mathrm{BC} 1$ & $\mid \overline{\hat{\alpha}_{n / m}}$ & 67 & 93 & 57 & 27 & 8.9 & & & & & & 46 & 70 & 24 & 10 & & & & & & & 57 & 86 & \begin{tabular}{|l|}
37 \\
\end{tabular} & 14 & \begin{tabular}{|l|l|}
4 & 7.9 \\
\end{tabular} & & & & & \\
\hline & $\hat{\beta}_{n / m}$ & 391 & 460 & 552 & 593 & 260 & & & & & & 303 & 393 & 376 & 317 & & & & & & & 348 & 462 & 382 & 297 & 7194 & & & & & \\
\hline $\mathrm{BC} 2$ & $\frac{\hat{\alpha}_{n / m}}{\hat{n_{1}}}$ & 10 & 7.6 & 17 & 4.6 & & & & & & & 8.8 & 22 & 10 & 2.6 & & & & & & & 11 & 10 & 14 & 4.8 & \begin{tabular}{|l|l}
8.3 \\
\end{tabular} & & & & & \\
\hline & $\hat{\beta}_{n / m}$ & 133 & 80 & 284 & 158 & & & & & & & 158 & 290 & 190 & 76 & & & & & & & 157 & 149 & 213 & 151 & 1110 & & & & & \\
\hline $\mathrm{BC} 3$ & $\overline{\hat{\alpha}}_{n / m}$ & 5.0 & 10 & 15 & 8.1 & 4.4 & 2.9 & & & & & 1.0 & 11 & 13 & 5.3 & 1.5 & & & & & & 3.0 & 8.1 & 7.9 & 5.0 & 4.5 & 3.6 & & & & \\
\hline & $\hat{\beta}_{n / m}$ & 87 & 125 & 192 & 154 & 121 & 92 & & & & & 25 & 213 & 244 & 147 & 26 & & & & & & 62 & 137 & 110 & 102 & 2122 & 96 & & & & \\
\hline $\mathrm{BC} 4$ & $\overline{\hat{\alpha}_{n / m}}$ & & & & 6.8 & 4.2 & 2.7 & & & & & & & & & & & & & & & & & & 3.6 & \begin{tabular}{|l|l}
5 & 3.1
\end{tabular} & 3.5 & 2.7 & & & \\
\hline & $\hat{\beta}_{n / m}$ & & & & 128 & 99 & 70 & & & & & & & & & & & & & & & & & & 76 & $5 \quad 67$ & 90 & 74 & & & \\
\hline $\mathrm{BC} 5$ & $\overline{\hat{\alpha}}_{n / m}$ & & & & 4.6 & & 3.4 & & & & & & & & 9.0 & & & & & & & & & & 6.7 & & 4.6 & 4.0 & & & \\
\hline & $\hat{\beta}_{n / m}$ & & & & 70 & & 81 & & & & & & & & 296 & & & & & & & & & & 147 & & 103 & 114 & & & \\
\hline BC6 & $\overline{\hat{\alpha}_{n / m}}$ & & & & & 2.4 & & 4.7 & & & & & & & & & & & & & & & & & & & & 3.8 & & & \\
\hline & $\hat{\beta}_{n / m}$ & & & & & 55 & & 89 & & & & & & & & & & & & & & & & & & & & 115 & & & \\
\hline BC7 & $\overline{\hat{\alpha}}_{n / m}$ & & & & & & & 1.7 & & & & & & & & & & & & & & & & & & & & & & & \\
\hline & $\hat{\hat{\beta}}_{n / m}$ & & & & & & & 19 & & & & & & & & & & & & & & & & & & & & & & & \\
\hline BC8 & $\hat{\hat{\alpha}}_{n / m}$ & & & & & & & & & & & & & & & & & & & & & & & & & & & & & & \\
\hline & $\hat{\hat{\beta}}_{n / m}$ & & & & & & & & & & & & & & & & & & & & & & & & & & & & & & \\
\hline BC9 & $\overline{\hat{\alpha}_{n / m}}$ & & & & & & & & & & & & & & & & & & & & & & & & & & & & & & \\
\hline & $\hat{\beta}_{n / m}$ & & & & & & & & & & & & & & & & & & & & & & & & & & & & & & \\
\hline $\mathrm{BC} 10$ & $\overline{\hat{\alpha}_{n / m}}$ & & & & & & & & & & & & & & & & & & & & & & & & & & & & & & \\
\hline & $\hat{\hat{\beta}}_{n / m}$ & & & & & & & & & & & & & & & & & & & & & & & & & & & & & & \\
\hline AC1 & $\underline{\hat{\alpha}_{n / m}}$ & & 10 & & 21 & & & & & & & & & & 13 & & & & & & & & & & 10 & & & & & & \\
\hline & $\hat{\hat{\beta}}_{n / m}$ & & 420 & & 569 & & & & & & & & & & 387 & & & & & & & & & & 329 & & & & & & \\
\hline AC2 & $\overline{\hat{\alpha}_{n / m}}$ & & & & & & & & & & & & & & & & & & & & & & & & & & & & & & \\
\hline & $\hat{\hat{\beta}}_{n / m}$ & & & & & & & & & & & & & & & & & & & & & & & & & & & & & & \\
\hline AC3 & $\overline{\hat{\alpha}_{n / m}}$ & & & 7.3 & & & & & & & & & & 2.2 & & & & & & & & & & & & & & & & & \\
\hline & $\hat{\beta}_{n / m}$ & & & 217 & & & & & & & & & & 61 & & & & & & & & & & & & & & & & & \\
\hline AC4 & $\underline{\hat{\alpha}_{n / m}}$ & & & & & & & & & & & & & & & & & & & & & & & & & & & & & & \\
\hline & $\widehat{\hat{\beta}}_{n / m}$ & & & & & & & & & & & & & & & & & & & & & & & & & & & & & & \\
\hline
\end{tabular}


Panel V: AC1 to AC10

\begin{tabular}{|c|c|c|c|c|c|c|c|c|c|c|c|c|c|c|c|c|c|c|c|c|c|c|c|c|c|c|c|c|c|c|}
\hline \multirow{2}{*}{\begin{tabular}{|l|l|} 
& \\
$n$ & $m$ \\
\end{tabular}} & \multicolumn{10}{|c|}{ BMW } & \multicolumn{10}{|c|}{ SAP } & \multicolumn{10}{|c|}{ ADS } \\
\hline & $\mathrm{AC1} A$ & $\mathrm{AC2} A$ & $\mathrm{AC} 3$ & AC4 & $\mathrm{AC5} \mathrm{A}$ & \begin{tabular}{l|l}
$A C 6$ \\
\end{tabular} & AC7 & $A C 8 A$ & \begin{tabular}{|l|l}
$A C 9$ & $A$ \\
\end{tabular} & $\mathrm{AC} 10$ & & $A C 2 A$ & \begin{tabular}{l|l}
$A C 3$ & $A$ \\
\end{tabular} & \begin{tabular}{l|l}
$\mathrm{AC} 4$ \\
\end{tabular} & $\mathrm{AC5}$ & $\mathrm{AC6}$ & AC7 & $\mathrm{AC} 8$ & AC9 & $\mathrm{AC} 10$ & \begin{tabular}{|l|l|l}
$A C 1$ & $A$ \\
\end{tabular} & \begin{tabular}{l|l}
$A C 2$ & $A$ \\
\end{tabular} & \begin{tabular}{l|l}
$\mathrm{AC} 3 \mathrm{~A}$ \\
\end{tabular} & \begin{tabular}{l|l}
$\mathrm{AC} 4$ & $\mathrm{~A}$ \\
\end{tabular} & \begin{tabular}{l|l}
$A C 5$ & $A$ \\
\end{tabular} & $\mathrm{AC6} \mathrm{A}$ & $\mathrm{AC7} A$ & $\mathrm{AC} 8 \mathrm{~A}$ & $A C 9 \mid A$ & $\mathrm{AC} 10$ \\
\hline Buy $* \frac{\overline{\hat{\alpha}_{n / m}}}{\hat{\hat{\beta}}_{n / m}}$ & \begin{tabular}{|l|l|}
117 & 1 \\
146 & 2 \\
\end{tabular} & \begin{tabular}{|l|l|}
170 & \\
200 & 1 \\
\end{tabular} & \begin{tabular}{r|}
72 \\
139 \\
\end{tabular} & $\begin{array}{r}36 \\
161 .\end{array}$ & \begin{tabular}{r|r}
22 & \\
126 & 1 \\
\end{tabular} & \begin{tabular}{r|r|}
19 & \\
182 & 1 \\
\end{tabular} & $\begin{array}{r}13 \\
110 \\
\end{array}$ & \begin{tabular}{|r|}
8.8 \\
83 \\
\end{tabular} & \begin{tabular}{|r|}
5.2 \\
88 \\
\end{tabular} & & \begin{tabular}{|l|}
104 \\
126 \\
\end{tabular} & \begin{tabular}{|l|l|}
170 & \\
185 & 1 \\
\end{tabular} & \begin{tabular}{r|r}
62 & \\
164 & 1 \\
\end{tabular} & \begin{tabular}{r|r}
34 & \\
161 & 1 \\
\end{tabular} & $\begin{array}{r}23 \\
157\end{array}$ & $\begin{array}{r}17 \\
124\end{array}$ & \begin{tabular}{r|}
10 \\
105 \\
\end{tabular} & & & & \begin{tabular}{|l|l|}
119 & 1 \\
162 & 1 \\
\end{tabular} & \begin{tabular}{l|l}
153 & \\
194 & 1 \\
\end{tabular} & \begin{tabular}{r|r}
71 & \\
156 & 1 \\
\end{tabular} & \begin{tabular}{r|r}
31 & \\
126 & 1 \\
\end{tabular} & \begin{tabular}{r|r}
21 & \\
106 & 1 \\
\end{tabular} & \begin{tabular}{r|r}
16 & \\
123 & 1 \\
\end{tabular} & \begin{tabular}{|l|}
15 \\
169
\end{tabular} & \begin{tabular}{r|r}
8.7 & 5 \\
76 & \\
\end{tabular} & \begin{tabular}{r|}
5.8 \\
92
\end{tabular} & \\
\hline 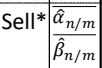 & & \begin{tabular}{|l|l}
10 & \\
98 & 1 \\
\end{tabular} & $\begin{array}{r}18 \\
141\end{array}$ & $\begin{array}{r}21 \\
150\end{array}$ & $\begin{array}{l}12 \\
79\end{array}$ & \begin{tabular}{r|}
7.7 \\
77
\end{tabular} & & & \begin{tabular}{r|}
7.1 \\
65
\end{tabular} & $\begin{array}{r}5.5 \\
37\end{array}$ & & \begin{tabular}{|r|r}
13 & \\
192 & 1 \\
\end{tabular} & \begin{tabular}{r|r}
20 & \\
138 & 1
\end{tabular} & $\begin{array}{r}22 \\
144\end{array}$ & $\begin{array}{l}14 \\
97\end{array}$ & \begin{tabular}{r|}
5.2 \\
40
\end{tabular} & & & & $\begin{array}{r}8.5 \\
56\end{array}$ & & \begin{tabular}{r|}
16 \\
101
\end{tabular} & $\begin{array}{l}11 \\
68\end{array}$ & $\begin{array}{l}16 \\
82\end{array}$ & \begin{tabular}{l|l}
12 & 9 \\
73 &
\end{tabular} & \begin{tabular}{r|}
9.5 \\
82
\end{tabular} & & & \begin{tabular}{r|}
5.6 \\
45
\end{tabular} & $\begin{array}{r}5.9 \\
38\end{array}$ \\
\hline \begin{tabular}{l|l|} 
Buy & $\frac{\overline{\hat{\alpha}}_{n / m}}{\hat{\beta}_{n / m}}$ \\
\end{tabular} & \begin{tabular}{l|l}
17 & \\
87 & \\
\end{tabular} & \begin{tabular}{r|r}
63 & \\
422 & 3 \\
\end{tabular} & $\begin{array}{r}50 \\
347\end{array}$ & $\begin{array}{r}28 \\
259\end{array}$ & \begin{tabular}{r|r}
13 & \\
178 & 1 \\
\end{tabular} & $\begin{array}{r}8.1 \\
139 \\
\end{array}$ & & & & & \begin{tabular}{l|}
11 \\
69
\end{tabular} & \begin{tabular}{|r|}
61 \\
369 \\
\end{tabular} & \begin{tabular}{r|r}
33 & \\
310 & 2 \\
\end{tabular} & \begin{tabular}{r|r}
13 & 7 \\
214 & 1 \\
\end{tabular} & \begin{tabular}{|l|}
7.8 \\
154
\end{tabular} & $\begin{array}{r}5.5 \\
98\end{array}$ & & & & & \begin{tabular}{l|l}
19 & \\
97 & 3 \\
\end{tabular} & \begin{tabular}{r|r}
51 \\
386 & 2 \\
\end{tabular} & \begin{tabular}{r|r}
37 & \\
276 & 2 \\
\end{tabular} & \begin{tabular}{r|r}
17 \\
216
\end{tabular} & & \begin{tabular}{r|}
5.3 \\
75 \\
\end{tabular} & & & & \\
\hline Sell $\begin{array}{l}\frac{\hat{\hat{\alpha}}_{n / m}}{\hat{\beta}_{n / m}} \\
\end{array}$ & & & & & & & & & & & & & & & & & & & & & & & & & & & & & & \\
\hline \begin{tabular}{|l|l|}
$B^{\prime} 1^{*}$ & $\overline{\hat{\alpha}_{n / m}}$ \\
$\hat{\beta}_{n / m}$ \\
\end{tabular} & \begin{tabular}{|l|}
117 \\
397 \\
\end{tabular} & \begin{tabular}{r|}
69 \\
3803 \\
\end{tabular} & $\begin{array}{r}57 \\
368 \\
\end{array}$ & $\begin{array}{r}24 \\
262\end{array}$ & \begin{tabular}{r|}
10 \\
150 \\
\end{tabular} & \begin{tabular}{|l|}
5.3 \\
133
\end{tabular} & & & & & \begin{tabular}{|l|}
112 \\
386 \\
\end{tabular} & \begin{tabular}{|r|r|}
63 & \\
294 & 2 \\
\end{tabular} & \begin{tabular}{r|r}
27 & \\
232 & 1 \\
\end{tabular} & \begin{tabular}{r|r}
12 & \\
172 & 1 \\
\end{tabular} & $\begin{array}{r}11 \\
118 \\
\end{array}$ & & & & & & \begin{tabular}{|r|r|}
67 & \\
267 & 2 \\
\end{tabular} & \begin{tabular}{r|r}
48 \\
256 \\
\end{tabular} & \begin{tabular}{r|r}
48 \\
283 \\
\end{tabular} & \begin{tabular}{r|r}
20 & 8 \\
210 & 1 \\
\end{tabular} & \begin{tabular}{l|l|}
8.3 & 6 \\
114 & \\
\end{tabular} & \begin{tabular}{r|}
6.0 \\
96 \\
\end{tabular} & \begin{tabular}{r|}
3.5 \\
83 \\
\end{tabular} & & & \\
\hline AA1* ${ }^{*} \frac{\overline{\hat{\alpha}_{n / m}}}{\hat{\beta}_{n / m}}$ & & \begin{tabular}{r|r}
55 \\
234 \\
\end{tabular} & $\begin{array}{r}26 \\
263 \\
\end{array}$ & $\begin{array}{l}130 \\
495 \\
\end{array}$ & $\begin{array}{r}35 \\
304 \\
\end{array}$ & $\begin{array}{r}9.0 \\
25 \\
\end{array}$ & $\begin{array}{l}11 \\
40\end{array}$ & \begin{tabular}{|r|r|}
9.3 & \\
50 & 1 \\
\end{tabular} & \begin{tabular}{r|}
14 \\
124 \\
\end{tabular} & $\begin{array}{r}36 \\
228 \\
\end{array}$ & & \begin{tabular}{|r|r}
61 & \\
244 & 1 \\
\end{tabular} & \begin{tabular}{r|l}
20 & 1 \\
196 & 4 \\
\end{tabular} & \begin{tabular}{l|}
118 \\
425 \\
\end{tabular} & $\begin{array}{l}14 \\
98 \\
\end{array}$ & \begin{tabular}{r|}
8.3 \\
22 \\
\end{tabular} & \begin{tabular}{|r|}
7.0 \\
24 \\
\end{tabular} & \begin{tabular}{|r|}
6.6 \\
43 \\
\end{tabular} & \begin{tabular}{|r|}
5.0 \\
49 \\
\end{tabular} & \begin{tabular}{r|}
16 \\
179 \\
\end{tabular} & & \begin{tabular}{l|l}
11 & \\
70 & 1 \\
\end{tabular} & \begin{tabular}{r|r}
17 & \\
156 & 3 \\
\end{tabular} & \begin{tabular}{r|r}
99 & \\
395 & 2 \\
\end{tabular} & \begin{tabular}{r|r}
21 & 7 \\
215 & \\
\end{tabular} & $\begin{array}{r}7.7 \\
36 \\
\end{array}$ & \begin{tabular}{r|r}
7.9 \\
42 \\
\end{tabular} & \begin{tabular}{r|r}
6.7 & 5 \\
46 & \\
\end{tabular} & $\begin{array}{r}9.1 \\
84 \\
\end{array}$ & $\begin{array}{r}27 \\
190 \\
\end{array}$ \\
\hline \begin{tabular}{l|l} 
BA1 & $\overline{\hat{\alpha}_{n / m}}$ \\
$\hat{\hat{\beta}}_{n / m}$
\end{tabular} & \begin{tabular}{|r|}
22 \\
313
\end{tabular} & \begin{tabular}{r|r}
39 \\
683
\end{tabular} & $\begin{array}{r}34 \\
694 \\
\end{array}$ & \begin{tabular}{r|}
13 \\
497
\end{tabular} & & & & & & & \begin{tabular}{|r|}
19 \\
280 \\
\end{tabular} & \begin{tabular}{|r|r|}
28 & \\
467 & 3 \\
\end{tabular} & $\begin{array}{r}12 \\
366\end{array}$ & & & & & & & & \begin{tabular}{|r|r}
17 & \\
278 & 3 \\
\end{tabular} & \begin{tabular}{r|r}
17 & \\
361 & 3 \\
\end{tabular} & \begin{tabular}{r|}
14 \\
342
\end{tabular} & & & & & & & \\
\hline \begin{tabular}{l|l} 
BA2 & $\overline{\hat{\alpha}_{n / m}}$ \\
$\hat{\beta}_{n / m}$
\end{tabular} & \begin{tabular}{|l|}
6.9 \\
179 \\
\end{tabular} & \begin{tabular}{|l|l}
7.5 & \\
234 & 2 \\
\end{tabular} & $\begin{array}{r}8.5 \\
291\end{array}$ & & & & & & & & \begin{tabular}{|l|}
5.5 \\
168 \\
\end{tabular} & \begin{tabular}{|r|}
3.4 \\
78 \\
\end{tabular} & & & & & & & & & \begin{tabular}{|l|l}
5.4 & 2 \\
153 & 1 \\
\end{tabular} & \begin{tabular}{r|r}
4.2 & 4 \\
115 & 1
\end{tabular} & \begin{tabular}{r|}
4.2 \\
125
\end{tabular} & & & & & & & \\
\hline 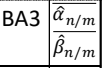 & & \begin{tabular}{r|}
2.1 \\
56 \\
\end{tabular} & $\begin{array}{r}2.9 \\
82 \\
\end{array}$ & & & & & & & & & \begin{tabular}{r|}
2.1 \\
47 \\
\end{tabular} & $\begin{array}{r}1.7 \\
50 \\
\end{array}$ & & & & & & & & \begin{tabular}{|r|r}
2.1 & 3 \\
57 & \\
\end{tabular} & \begin{tabular}{r|l}
3.5 & 4 \\
89 & 1 \\
\end{tabular} & $\begin{array}{r}4.5 \\
113 \\
\end{array}$ & & & & & & & \\
\hline \begin{tabular}{l|l} 
AA1 & $\underline{\hat{\alpha}_{n / m}}$ \\
$\hat{\hat{\beta}}_{n / m}$
\end{tabular} & \begin{tabular}{|r|r}
5.2 & \\
57 & 8 \\
\end{tabular} & \begin{tabular}{|r|r|}
26 \\
856
\end{tabular} & & \begin{tabular}{r|}
14 \\
515
\end{tabular} & & & & & & & \begin{tabular}{|r|}
3.1 \\
20 \\
\end{tabular} & & & & & & & & & & \begin{tabular}{|r|}
3.8 \\
52 \\
\end{tabular} & & & & & & & & & \\
\hline 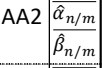 & & $\begin{array}{r}9.3 \\
279 \\
\end{array}$ & & & & & & & & & & \begin{tabular}{|l|}
1.0 \\
6.8 \\
\end{tabular} & & & & & & & & & & & & & & & & & & \\
\hline AA3 $\frac{\overline{\hat{\alpha}_{n / m}}}{\frac{\hat{\beta}_{n / m}}{n}}$ & & & $\begin{array}{r}3.9 \\
105 \\
\end{array}$ & & & & & & & & & & $\begin{array}{l}1.1 \\
7.6\end{array}$ & & & & & & & & & & & & & & & & & \\
\hline \begin{tabular}{l|l} 
AA4 & $\frac{\overline{\hat{\alpha}}_{n / m}}{\hat{\hat{\beta}}_{n / m}}$ \\
\end{tabular} & & & & & & & & & & & & & & $\begin{array}{r}2.5 \\
44 \\
\end{array}$ & & & & & & & & & & & & & & & & \\
\hline $\begin{array}{l}\text { AA5 } \frac{\overline{\hat{\hat{\alpha}}_{n / m}}}{\frac{\hat{\beta}_{n / m}}{n_{n / m}}} \\
\end{array}$ & & & & & \begin{tabular}{l|}
0.3 \\
2.7
\end{tabular} & & & & & & & & & & $\begin{array}{r}2.7 \\
48\end{array}$ & & & & & & & & & & & & & & & \\
\hline AA6 $\begin{array}{l}\frac{\hat{\hat{\alpha}}_{n / m}}{\frac{\hat{\beta}_{n / m}}{n_{n / m}}} \\
\end{array}$ & & & & & & & & & & & & & & & & $\begin{array}{l}0.5 \\
1.6 \\
\end{array}$ & & & & & & & & & & & & & & \\
\hline BC1 $\mid$\begin{tabular}{l|}
$\frac{\hat{\alpha}_{n / m}}{\hat{\beta}_{n / m}}$ \\
\end{tabular} & & $\begin{array}{r}11 \\
411 \\
\end{array}$ & & $\begin{array}{r}20 \\
607 \\
\end{array}$ & & & & & & & & & & $\begin{array}{r}13 \\
373 \\
\end{array}$ & & & & & & & & & & & & & & & & \\
\hline \begin{tabular}{l|l} 
BC2 & $\begin{array}{l}\hat{\hat{\alpha}}_{n / m} \\
\hat{\beta}_{n / m}\end{array}$ \\
\end{tabular} & & & & & & & & & & & & & & & & & & & & & & & & & & & & & & \\
\hline \begin{tabular}{l|l} 
BC3 & $\overline{\hat{\alpha}_{n / m}}$ \\
$\hat{\beta}_{n / m}$
\end{tabular} & & & \begin{tabular}{|c|}
7.3 \\
219
\end{tabular} & & & & & & & & & & & & & & & & & & & & & & & & & & & \\
\hline \begin{tabular}{l|l|} 
BC4 & $\frac{\hat{\hat{\alpha}}_{n / m}}{\hat{\beta}_{n / m}}$ \\
\end{tabular} & & & & & & & & & & & & & & $\begin{array}{r}1.0 \\
42 \\
\end{array}$ & & & & & & & & & & & & & & & & \\
\hline AC1 $\mid \begin{array}{l}\frac{\hat{\hat{\alpha}}_{n / m}}{\hat{\beta}_{n / m}} \\
\end{array}$ & \begin{tabular}{|r|r}
64 & \\
354 & 4 \\
\end{tabular} & \begin{tabular}{r|r}
93 & \\
455 & 5 \\
\end{tabular} & $\begin{array}{r}56 \\
572 \\
\end{array}$ & $\begin{array}{r}26 \\
676\end{array}$ & \begin{tabular}{r|}
8.9 \\
275 \\
\end{tabular} & & & & & & \begin{tabular}{|r|}
41 \\
272 \\
\end{tabular} & \begin{tabular}{|r|r}
68 & \\
365 & 3 \\
\end{tabular} & \begin{tabular}{r|r}
24 & 6 \\
364 & 1 \\
\end{tabular} & $\begin{array}{r}6.0 \\
173 \\
\end{array}$ & & & & & & & \begin{tabular}{|r|r}
58 & \\
352 & 4 \\
\end{tabular} & \begin{tabular}{r|r}
89 & 3 \\
465 & 35
\end{tabular} & \begin{tabular}{r|r}
38 & \\
397 & 3 \\
\end{tabular} & \begin{tabular}{r|r}
14 & 7 \\
310 & 1 \\
\end{tabular} & \begin{tabular}{r|}
7.8 \\
192 \\
\end{tabular} & & & & & \\
\hline 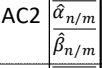 & \begin{tabular}{|r|}
8.0 \\
95 \\
\end{tabular} & \begin{tabular}{r|}
7.3 \\
792 \\
\end{tabular} & $\begin{array}{r}16 \\
282\end{array}$ & $\begin{array}{r}4.2 \\
160 \\
\end{array}$ & & & & & & & \begin{tabular}{|l|}
7.9 \\
127 \\
\end{tabular} & \begin{tabular}{r|r}
14 \\
166 & 2 \\
\end{tabular} & \begin{tabular}{r|l}
11 & 3 \\
232 & \\
\end{tabular} & $\begin{array}{r}3.5 \\
97 \\
\end{array}$ & & & & & & & \begin{tabular}{|c|c|}
8.8 & 5 \\
134 & 1 \\
\end{tabular} & \begin{tabular}{r|r}
9.1 & \\
119 & 2 \\
\end{tabular} & \begin{tabular}{r|r}
14 & 5 \\
212 & 1 \\
\end{tabular} & \begin{tabular}{r|r}
5.0 & 3 \\
153 & 1 \\
\end{tabular} & $\begin{array}{r}3.5 \\
117 \\
\end{array}$ & & & & & \\
\hline AC3 $\begin{array}{l}\frac{\hat{\alpha}_{n / m}}{\hat{\hat{\beta}}_{n / m}} \\
\end{array}$ & \begin{tabular}{|r|r}
3.4 & \\
51 & 1 \\
\end{tabular} & \begin{tabular}{r|r}
9.3 & \\
117 & 1 \\
\end{tabular} & $\begin{array}{r}16 \\
185 \\
\end{array}$ & $\begin{array}{r}8.3 \\
160\end{array}$ & \begin{tabular}{r|r}
4.6 & \\
125 & 1
\end{tabular} & $\begin{array}{r}3.0 \\
100 \\
\end{array}$ & & & & & $\begin{array}{r}1.7 \\
49 \\
\end{array}$ & \begin{tabular}{r|}
15 \\
254 \\
2
\end{tabular} & \begin{tabular}{r|l}
13 & 6 \\
230 & 1 \\
\end{tabular} & \begin{tabular}{r|r}
6.2 & 1 \\
170 & \\
\end{tabular} & $\begin{array}{r}1.5 \\
27 \\
\end{array}$ & & & & & & \begin{tabular}{|r|l|}
2.6 & 8 \\
53 & 1 \\
\end{tabular} & \begin{tabular}{r|r}
8.1 & 7 \\
136 & 5 \\
\end{tabular} & \begin{tabular}{r|r}
7.3 & 5 \\
98 & 1 \\
\end{tabular} & \begin{tabular}{r|r}
5.8 & 4 \\
117 & 1 \\
\end{tabular} & \begin{tabular}{r|r}
4.0 & 3 \\
109 & \\
\end{tabular} & $\begin{array}{r}3.5 \\
98 \\
\end{array}$ & & & & \\
\hline AC4 $\frac{\overline{\hat{\alpha}_{n / m}}}{\hat{\hat{\beta}}_{n / m}}$ & & & & \begin{tabular}{|c|}
5.7 \\
101
\end{tabular} & $\begin{array}{r}4.2 \\
96 \\
\end{array}$ & $\begin{array}{r}3.1 \\
80 \\
\end{array}$ & & & & & & & & & $\begin{array}{c}3.2 \\
106\end{array}$ & & & & & & & & & \begin{tabular}{r|r}
4.0 & 3 \\
73 & \\
\end{tabular} & \begin{tabular}{r|r}
3.1 & 3 \\
68 & \\
\end{tabular} & \begin{tabular}{r|}
3.7 \\
93
\end{tabular} & \begin{tabular}{r|}
3.0 \\
82
\end{tabular} & & & \\
\hline AC5 $\frac{\underline{\hat{\hat{\alpha}}_{n / m}}}{\hat{\hat{\beta}}_{n / m}}$ & & & & \begin{tabular}{r|}
3.7 \\
58 \\
\end{tabular} & & \begin{tabular}{r|}
3.8 \\
87
\end{tabular} & & & & & & & & & & & & & & & & & & \begin{tabular}{|l|}
5.6 \\
122
\end{tabular} & & \begin{tabular}{r|r}
4.9 & \\
105 & 1 \\
\end{tabular} & \begin{tabular}{l|}
4.2 \\
122
\end{tabular} & & & \\
\hline \begin{tabular}{l|l} 
AC6 & $\frac{\hat{\hat{\alpha}}_{n / m}}{\hat{\hat{\beta}}_{n / m}}$ \\
\end{tabular} & & & & & \begin{tabular}{r|}
1.7 \\
34 \\
\end{tabular} & & $\begin{array}{r}5.9 \\
100 \\
\end{array}$ & & & & & & & & & & & & & & & & & & & & $\begin{array}{l}5.0 \\
143 \\
\end{array}$ & & & \\
\hline AC7 $\frac{\hat{\hat{\alpha}}_{n / m}}{\frac{\hat{\beta}_{n / m}}{\hat{n}^{2}}}$ & & & & & & & $\begin{array}{r}1.3 \\
15 \\
\end{array}$ & & & & & & & & & & & & & & & & & & & & & & & \\
\hline AC8 $\begin{array}{l}\frac{\hat{\hat{\alpha}}_{n / m}}{\hat{\hat{\beta}}_{n / m}} \\
\end{array}$ & & & & & & & & & & & & & & & & & & & & & & & & & & & & & & \\
\hline AC9 $\begin{array}{ll}\frac{\hat{\alpha}_{n / m}}{\hat{\beta}_{n / m}} \\
\end{array}$ & & & & & & & & & & & & & & & & & & & & & & & & & & & & & & \\
\hline AC10 $\frac{\hat{\underline{\hat{\alpha}}}_{n / m}}{\hat{\beta}_{n / m}}$ & & & & & & & & & \begin{tabular}{r|}
1.4 \\
15 \\
\end{tabular} & & & & & & & & & & & & & & & & & & & & & \\
\hline
\end{tabular}


Panel VI: BA11 to BA20, AA11 to AA20, BC11 to BC20 and AC11 to AC20

\begin{tabular}{|c|c|c|c|c|c|c|c|c|c|c|c|c|c|c|c|c|c|c|c|c|c|c|c|c|c|c|c|c|c|c|}
\hline \multirow{3}{*}{\begin{tabular}{|l|} 
\\
$\underline{n} \quad m$ \\
Buy*
\end{tabular}} & \multicolumn{10}{|c|}{ BMW } & \multicolumn{10}{|c|}{ SAP } & \multicolumn{10}{|c|}{ ADS } \\
\hline & BA11 & BA12 & BA13 & BA14 & BA15 & BA16 & BA17 & BA18 & BA19 & BA20 & BA11 & BA12 & BA13 & \begin{tabular}{|l|l|l|} 
BA14 & \\
\end{tabular} & BA15B & BA16 & BA17 & BA18 & BA19 & BA20 & BA11 & BA12 & BA13 & BA14 & \begin{tabular}{ll|l|}
4 BA15 \\
\end{tabular} & BA16 & \begin{tabular}{|l|l|}
6 BA17 \\
\end{tabular} & BA18 & BA19 & BA20 \\
\hline & & & & & & & & & & & & & & & & & & & & & & & & & & & & & & \\
\hline Sell $* \frac{\overline{\hat{\alpha}_{n / m}}}{\frac{\hat{\beta}_{n / m}}{2}}$ & $\begin{array}{r}5.4 \\
66\end{array}$ & & \begin{tabular}{|r|}
1.4 \\
26 \\
\end{tabular} & \begin{tabular}{|r|}
3.3 \\
41 \\
\end{tabular} & \begin{tabular}{|r|}
7.6 \\
67
\end{tabular} & $\begin{array}{r}3.9 \\
88\end{array}$ & & & & & $\begin{array}{r}3.3 \\
52\end{array}$ & $\begin{array}{r}0.8 \\
20\end{array}$ & $\begin{array}{r}2.6 \\
51\end{array}$ & $\begin{array}{r}4.7 \\
64\end{array}$ & $\begin{array}{r}7.0 \\
74\end{array}$ & $\begin{array}{r}3.1 \\
67\end{array}$ & & & & & $\begin{array}{r}6.3 \\
62\end{array}$ & $\begin{array}{r}3.4 \\
39\end{array}$ & $\begin{array}{r}1.8 \\
26\end{array}$ & \begin{tabular}{|r|}
3.0 \\
35
\end{tabular} & \begin{tabular}{|r|r|}
6.3 \\
56
\end{tabular} & $\begin{array}{r}6.8 \\
63\end{array}$ & & & & \\
\hline \begin{tabular}{|l|l|} 
Buy & $\overline{\hat{\alpha}_{n / m}}$ \\
$\hat{\beta}_{n / m}$
\end{tabular} & & & & & & & & & & & & & & & & & & & & & & & & & & & & & & \\
\hline \begin{tabular}{|l|l|} 
Sell & $\frac{\overline{\hat{\alpha}_{n / m}}}{\frac{\hat{\beta}_{n / m}}{}}$ \\
\end{tabular} & \begin{tabular}{|r|}
24 \\
351 \\
\end{tabular} & & & & & & & & & & $\begin{array}{r}25 \\
413 \\
\end{array}$ & & & & & & & & & & $\begin{array}{r}24 \\
310 \\
\end{array}$ & & & & & & & & & \\
\hline BA1* $\sqrt{\frac{\hat{\alpha}_{n / m}}{\hat{\beta}_{n / m}}}$ & & & & & & & & & & & & & & & & & & & & & & & & & & & & & & \\
\hline AA1 $*$\begin{tabular}{|l|}
$\frac{\overline{\hat{\alpha}_{n / m}}}{\hat{\beta}_{n / m}}$ \\
\end{tabular} & & & & & & & & & & & & & & & & & & & & & & & & & & & & & & \\
\hline $\begin{array}{ll}n & m \\
\end{array}$ & AA11 & AA12A & AA13 & AA14 & AA15 & AA16 & AA17 & AA18 & AA19 & AA20 & AA11 $A$ & AA12 & AA13A & AA14A & AA15 A & AA16 & AA17 & AA18 & AA19 & AA20 & AA11 & AA12 & AA13 & AA14 & 4 AA15 & AA16 & 6 AA17 & AA18 & AA19A & $\mathrm{AA} 20$ \\
\hline \begin{tabular}{|l|l|} 
Buy & $\frac{\hat{\hat{\alpha}}_{n / m}}{\hat{\beta}_{n / m}}$ \\
\end{tabular} & \begin{tabular}{r|}
5.2 \\
66 \\
\end{tabular} & \begin{tabular}{r|}
2.3 \\
35 \\
\end{tabular} & & \begin{tabular}{|r|}
3.2 \\
43 \\
\end{tabular} & \begin{tabular}{r|}
7.3 \\
68 \\
\end{tabular} & $\begin{array}{r}5.7 \\
73 \\
\end{array}$ & & & & & \begin{tabular}{|r|}
3.4 \\
56
\end{tabular} & & $\begin{array}{r}2.6 \\
54\end{array}$ & $\begin{array}{r}4.5 \\
68\end{array}$ & $\begin{array}{r}6.7 \\
76\end{array}$ & \begin{tabular}{|r|}
3.3 \\
79
\end{tabular} & & & & & \begin{tabular}{|r|}
5.9 \\
65
\end{tabular} & & $\begin{array}{r}1.8 \\
29\end{array}$ & $\begin{array}{r}3.3 \\
38\end{array}$ & \begin{tabular}{|r|r|} 
& 6.3 \\
& 58
\end{tabular} & $\begin{array}{r}6.9 \\
66\end{array}$ & & & & \\
\hline Sell $* \frac{\overline{\hat{\alpha}_{n / m}}}{\hat{\beta}_{n / m}}$ & & & & & & & & & & & & & & & & & & & & & & & & & & & & & & \\
\hline \begin{tabular}{|l|l|} 
Buy & $\begin{array}{l}\hat{\alpha}_{n / m} \\
\frac{\hat{\beta}_{n / m}}{}\end{array}$ \\
\end{tabular} & & & & & & & & & & & & & & & & & & & & & & & & & & & & & & \\
\hline \begin{tabular}{|l|l|} 
Sell & $\overline{\hat{\alpha}_{n / m}}$ \\
$\hat{\beta}_{n / m}$ \\
\end{tabular} & & & & & & & & & & & & & & & & & & & & & & & & & & & & & & \\
\hline BA1* $\frac{\hat{\hat{\alpha}}_{n / m}}{\frac{\hat{\beta}_{n / m}}{y^{\prime}}}$ & & & & & & & & & & & & & & & & & & & & & & & & & & & & & & \\
\hline \begin{tabular}{|l|l|} 
AA1 $* \frac{\hat{\alpha}_{n / m}}{\frac{\hat{\beta}_{n / m}}{2}}$ \\
\end{tabular} & & & & & & & & & & & & & & & & & & & & & & & & & & & & & & \\
\hline$n-m$ & BC11 & $B C 12$ & $\mathrm{BC} 13$ & BC14 & $\mathrm{BC} 15$ & $\mathrm{BC} 16$ & $\mathrm{BC} 17$ & $\mathrm{BC} 18$ & BC19 & $B C 20$ & BC11 & $\mathrm{BC} 12$ & $\mathrm{BC} 13 \mathrm{~B}$ & BC14 & BC15 & BC16 & $\mathrm{BC} 17$ & $\mathrm{BC} 18$ & BC19 & $\mathrm{BC} 20$ & BC11 & BC12 & $B C 13$ & $\mathrm{BC} 14$ & BC15 & BC16 & BC17 & BC18 & BC19 & BC20 \\
\hline \begin{tabular}{|l|} 
Buy* \\
$\frac{\hat{\alpha}_{n / m}}{\hat{\beta}_{n / m}}$
\end{tabular} & $\begin{array}{r}7.5 \\
40\end{array}$ & $\begin{array}{r}7.1 \\
54\end{array}$ & $\begin{array}{r}4.0 \\
42 \\
\end{array}$ & $\begin{array}{r}3.9 \\
50\end{array}$ & \begin{tabular}{|r|}
5.2 \\
62
\end{tabular} & $\begin{array}{r}5.8 \\
60\end{array}$ & & & & & $\begin{array}{r}2.6 \\
23\end{array}$ & $\begin{array}{r}3.1 \\
42\end{array}$ & $\begin{array}{r}1.8 \\
17\end{array}$ & $\begin{array}{r}2.5 \\
23\end{array}$ & $\begin{array}{r}2.7 \\
40\end{array}$ & \begin{tabular}{|r|}
5.5 \\
83
\end{tabular} & $\begin{array}{r}3.9 \\
86\end{array}$ & & & & \begin{tabular}{|r|}
8.3 \\
43
\end{tabular} & $\begin{array}{r}7.9 \\
61\end{array}$ & $\begin{array}{r}4.0 \\
43\end{array}$ & & $\begin{array}{r}4.4 \\
63\end{array}$ & $\begin{array}{r}5.1 \\
59\end{array}$ & $\begin{array}{r}4.6 \\
51\end{array}$ & $\begin{array}{r}3.8 \\
53\end{array}$ & & \\
\hline Sell $* \frac{\hat{\alpha}_{n / m}}{\frac{\hat{\beta}_{n / m}}{2}}$ & & & & & & & & & & & & & & & & & & & & & & & & & & & & & & \\
\hline \begin{tabular}{|l|l|} 
Buy & $\frac{\hat{\alpha}_{n / m}}{\hat{\beta}_{n / m}}$ \\
\end{tabular} & & & & & & & & & & & & & & & & & & & & & & & & & & & & & & \\
\hline \begin{tabular}{|l|l|} 
Sell & $\frac{\hat{\alpha}_{n / m}}{\hat{\beta}_{n / m}}$ \\
\end{tabular} & & & & & & & & & & & & & & & & & & & & & & & & & & & & & & \\
\hline \begin{tabular}{|l|l|} 
BA1 & $\frac{\hat{\alpha}_{n / m}}{\hat{\beta}_{n / m}}$ \\
\end{tabular} & $\begin{array}{l}362 \\
578\end{array}$ & \begin{tabular}{r|}
12 \\
148 \\
\end{tabular} & \begin{tabular}{|r|}
4.8 \\
80 \\
\end{tabular} & \begin{tabular}{|r|}
2.5 \\
44 \\
\end{tabular} & $\begin{array}{r}2.3 \\
55\end{array}$ & $\begin{array}{r}3.8 \\
72\end{array}$ & $\begin{array}{r}4.8 \\
55 \\
\end{array}$ & \begin{tabular}{|r|}
2.2 \\
28
\end{tabular} & \begin{tabular}{|r|}
0.6 \\
15 \\
\end{tabular} & & $\begin{array}{l}322 \\
532\end{array}$ & \begin{tabular}{|r|}
3.0 \\
57 \\
\end{tabular} & & & $\begin{array}{r}3.1 \\
50 \\
\end{array}$ & \begin{tabular}{r|}
2.3 \\
35 \\
\end{tabular} & \begin{tabular}{|r|}
2.8 \\
82
\end{tabular} & & & & $\begin{array}{l}324 \\
513\end{array}$ & $\begin{array}{r}13 \\
148\end{array}$ & \begin{tabular}{|r|}
4.8 \\
86
\end{tabular} & \begin{tabular}{|r|}
2.7 \\
44 \\
\end{tabular} & \begin{tabular}{|r|}
2.0 \\
55
\end{tabular} & $\begin{array}{r}2.8 \\
66 \\
\end{array}$ & \begin{tabular}{|r|}
4.7 \\
84 \\
\end{tabular} & $\begin{array}{r}3.8 \\
65 \\
\end{array}$ & $\begin{array}{r}2.5 \\
39 \\
\end{array}$ & \\
\hline \begin{tabular}{|l|l|} 
AA1 & $\frac{\hat{\alpha}_{n / m}}{\hat{\beta}_{n / m}}$ \\
\end{tabular} & & & & & & & & & & & & & & & & & & & & & & & & & & & & & & \\
\hline $\begin{array}{ll}n & m\end{array}$ & AC11 & AC12 & AC13 & AC14 & AC15 & AC16 & AC17 & AC18 & AC19 & $A C 20$ & AC11 & $\mathrm{AC12}$ & $\mathrm{AC} 13 \mathrm{~A}$ & AC14 & AC15A & AC16 & AC17 & AC18 & AC19 & AC20 & AC11 & AC12 & AC13 & AC14 & AC15 & AC16 & 6 AC17 & AC18A & AC19A & $\mathrm{AC} 20$ \\
\hline \begin{tabular}{|l|} 
Buy* \\
$\frac{\hat{\alpha}_{n / m}}{\hat{\beta}_{n / m}}$ \\
\end{tabular} & & & & & & & & & & & & & & & & & & & & & & & & & & & & & & \\
\hline \begin{tabular}{|l|l|} 
Sell & $\frac{\hat{\alpha}_{n / m}}{\hat{\beta}_{n / m}}$ \\
\end{tabular} & $\begin{array}{r}7.4 \\
40 \\
\end{array}$ & $\begin{array}{r}7.8 \\
61 \\
\end{array}$ & \begin{tabular}{r|}
4.5 \\
46 \\
\end{tabular} & \begin{tabular}{|r|}
4.3 \\
32 \\
\end{tabular} & $\begin{array}{r}4.7 \\
39\end{array}$ & $\begin{array}{r}6.0 \\
63 \\
\end{array}$ & \begin{tabular}{|r|}
3.9 \\
54 \\
\end{tabular} & & & & $\begin{array}{r}2.5 \\
21 \\
\end{array}$ & \begin{tabular}{|r|}
3.7 \\
47 \\
\end{tabular} & $\begin{array}{r}2.1 \\
31 \\
\end{array}$ & $\begin{array}{r}2.8 \\
25 \\
\end{array}$ & $\begin{array}{r}3.0 \\
40 \\
\end{array}$ & \begin{tabular}{|r|}
6.5 \\
83 \\
\end{tabular} & \begin{tabular}{|r|}
3.9 \\
76 \\
\end{tabular} & & & & $\begin{array}{r}8.7 \\
44 \\
\end{array}$ & $\begin{array}{r}7.9 \\
59 \\
\end{array}$ & $\begin{array}{r}3.9 \\
41 \\
\end{array}$ & \begin{tabular}{|r|}
3.8 \\
50 \\
\end{tabular} & \begin{tabular}{|r|}
4.1 \\
55 \\
\end{tabular} & \begin{tabular}{|r|}
5.7 \\
59 \\
\end{tabular} & \begin{tabular}{|r|}
4.9 \\
54 \\
\end{tabular} & & & \\
\hline \begin{tabular}{|l|l|} 
Buy & $\frac{\hat{\alpha}_{n / m}}{\hat{\beta}_{n / m}}$ \\
\end{tabular} & & & & & & & & & & & & & & & & & & & & & & & & & & & & & & \\
\hline \begin{tabular}{|l|l|} 
Sell & $\frac{\hat{\alpha}_{n / m}}{\hat{\beta}_{n / m}}$ \\
\end{tabular} & & & & & & & & & & & & & & & & & & & & & & & & & & & & & & \\
\hline \begin{tabular}{|l|l|} 
BA1 & $\frac{\hat{\alpha}_{n / m}}{\hat{\beta}_{n / m}}$ \\
\end{tabular} & & & & & & & & & & & & & & & & & & & & & & & & & & & & & & \\
\hline 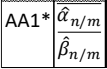 & $\begin{array}{l}353 \\
570\end{array}$ & \begin{tabular}{r|}
11 \\
137 \\
\end{tabular} & \begin{tabular}{|r|}
3.8 \\
64 \\
\end{tabular} & & & $\begin{array}{r}3.4 \\
69 \\
\end{array}$ & $\begin{array}{r}4.5 \\
66 \\
\end{array}$ & \begin{tabular}{|r|}
2.3 \\
31 \\
\end{tabular} & \begin{tabular}{|r|}
0.6 \\
16 \\
\end{tabular} & & $\begin{array}{l}317 \\
510\end{array}$ & $\begin{array}{r}2.8 \\
59 \\
\end{array}$ & \begin{tabular}{r|}
1.3 \\
26 \\
\end{tabular} & & \begin{tabular}{|r|}
3.3 \\
50 \\
\end{tabular} & \begin{tabular}{|r|}
2.1 \\
29 \\
\end{tabular} & \begin{tabular}{|r|}
2.5 \\
73 \\
\end{tabular} & & & & $\begin{array}{l}323 \\
510 \\
\end{array}$ & $\begin{array}{r}11 \\
141 \\
\end{array}$ & $\begin{array}{r}4.6 \\
86 \\
\end{array}$ & \begin{tabular}{|r|}
2.0 \\
47 \\
\end{tabular} & $\begin{array}{r}2.3 \\
61 \\
\end{array}$ & \begin{tabular}{|r|}
2.6 \\
62 \\
\end{tabular} & \begin{tabular}{|r|}
4.7 \\
77 \\
\end{tabular} & \begin{tabular}{r|}
4.4 \\
58 \\
\end{tabular} & \begin{tabular}{|r|}
2.6 \\
41 \\
\end{tabular} & \\
\hline
\end{tabular}




\section{Table 5 Depth Levels 1 to 11 descriptive models effects metrics}

Panel I: Trades and LOS w/ BPI

\begin{tabular}{|c|c|c|c|c|c|c|c|c|c|c|c|c|c|c|c|c|c|c|}
\hline & & & & MW & & & & & SA & AP & & & & & & DS & & \\
\hline & Buy* & Sell* & Buy & Sell & BA1* & AA1* & Buy* & Sell* & \begin{tabular}{|l|} 
Buy \\
\end{tabular} & Sell & BA1* & AA1* & Buy* & Sell* & Buy & Sell & BA1* & AA1* \\
\hline Buy* & & & & & 0.27 & 0.23 & & & & & 0.27 & 0.17 & & & & & 0.29 & 0.19 \\
\hline & & & & & 0.11 & 0.10 & & & & & 0.13 & 0.08 & & & & & 0.11 & 0.07 \\
\hline Sell* & & & & & 0.18 & 0.28 & & & & & 0.17 & 0.28 & & & & & 0.18 & 0.31 \\
\hline & & & & & 0.07 & 0.12 & & & & & 0.08 & 0.14 & & & & & 0.07 & 0.11 \\
\hline Buy & \begin{tabular}{|l|}
0.22 \\
\end{tabular} & & 0.20 & & 0.13 & & 0.17 & & 0.22 & & 0.10 & & 0.20 & & 0.21 & & 0.13 & \\
\hline & 0.12 & & 0.20 & & 0.03 & & 0.13 & & 0.22 & & 0.04 & & 0.11 & & 0.21 & & 0.03 & \\
\hline Sell & & 0.23 & & 0.23 & & 0.13 & & 0.18 & & 0.23 & & 0.11 & & 0.21 & & 0.20 & & 0.15 \\
\hline & & 0.12 & & 0.23 & & 0.03 & & 0.13 & & 0.23 & & 0.04 & & 0.11 & & 0.20 & & 0.03 \\
\hline BA1* & 0.06 & \begin{tabular}{|l|}
0.08 \\
\end{tabular} & & 0.04 & 0.06 & & & 0.10 & & 0.06 & & & \begin{tabular}{|l|}
0.06 \\
\end{tabular} & \begin{tabular}{|l|}
0.06 \\
\end{tabular} & & & \begin{tabular}{|l|}
0.07 \\
\end{tabular} & \\
\hline & 0.14 & 0.19 & & 0.17 & 0.06 & & & 0.21 & & 0.16 & & & 0.15 & 0.17 & & & 0.07 & \\
\hline AA1* & 0.08 & 0.06 & 0.04 & & & 0.07 & 0.10 & & 0.06 & & & & \begin{tabular}{|l|}
0.07 \\
\end{tabular} & 0.06 & & & & 0.06 \\
\hline & 0.19 & 0.14 & 0.17 & & & 0.07 & 0.21 & & 0.16 & & & & \begin{tabular}{|l|}
0.18 \\
\end{tabular} & 0.16 & & & & 0.06 \\
\hline BA1 & & & & & 0.05 & & & & & & 0.05 & & & & & & 0.05 & \\
\hline & & & & & 0.15 & & & & & & 0.16 & & & & & & 0.11 & \\
\hline AA1 & & & & & & 0.06 & & & & & & 0.05 & & & & & & 0.05 \\
\hline & & & & & & 0.18 & & & & & & 0.17 & & & & & & 0.11 \\
\hline BC1 & & & & & 0.04 & 0.05 & & & & & 0.05 & 0.03 & & & & & 0.06 & 0.05 \\
\hline & & & & & 0.12 & 0.14 & & & & & 0.17 & 0.13 & & & & & 0.14 & 0.12 \\
\hline AC1 & & & & & 0.04 & & & & & & 0.04 & 0.05 & & & & & 0.05 & 0.05 \\
\hline & & & & & $\mid 0.14$ & & & & & & 0.12 & $\mid$\begin{tabular}{|l|l}
0.17 \\
\end{tabular} & & & & & $\mid 0.12$ & 0.14 \\
\hline
\end{tabular}

For each Hawkes effect, this figure presents the daily average branching ratio and adjusted branching ratio. 


\section{Panel II: Trades and LOS w/ BPI effect on LOB events}

\begin{tabular}{|c|c|c|c|c|c|c|c|c|c|c|c|c|c|c|c|c|c|c|c|c|c|c|c|c|c|c|c|c|c|c|c|c|c|}
\hline & & & & & & BMW & & & & & & & & & & & SAP & & & & & & & & & & & ADS & & & & & \\
\hline & BA1 & 1 BA2 & BA3 & BA4 & BA5 & BA6 & BA7 & BA8 & BA99 & BA10 & OBA11 & BA1 & BA2 & BA3 & BA4 & BA5 & BA6 & BA7 & BA8 & BA9 & BA10 & BA11 B & BA1 & BA2 & BA3 & 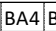 & BA5 & BA6 & BA7 & BA8 & BA9 & BA10 & BA11 \\
\hline Buy* & \begin{tabular}{l|}
0.45 \\
0.06
\end{tabular} & \begin{tabular}{|l|l}
5 & 0.34 \\
0.06
\end{tabular} & \begin{tabular}{|l|}
0.31 \\
0.04
\end{tabular} & \begin{tabular}{|l|}
0.22 \\
0.03 \\
\end{tabular} & $\begin{array}{l}0.18 \\
0.05\end{array}$ & & & & & & & \begin{tabular}{|l|l|l|}
0.44 & 0 \\
0.06 & 0
\end{tabular} & $\begin{array}{l}0.36 \\
0.05\end{array}$ & \begin{tabular}{|l|}
0.33 \\
0.03
\end{tabular} & $\begin{array}{l}0.16 \\
0.02\end{array}$ & & & & & & & & \begin{tabular}{|l|}
0.40 \\
0.06
\end{tabular} & \begin{tabular}{|l|}
0.35 \\
0.07
\end{tabular} & $\begin{array}{l}0.38 \\
0.06\end{array}$ & \begin{tabular}{|l|l|}
0.24 & 0 \\
0.04 & 0 \\
\end{tabular} & & & & & & & \\
\hline Sell* & \begin{tabular}{|l|}
0.32 \\
0.04
\end{tabular} & $\begin{array}{ll}2 & 0.14 \\
4 & 0.02\end{array}$ & \begin{tabular}{|l|}
0.26 \\
0.03
\end{tabular} & \begin{tabular}{|l|}
0.17 \\
0.02
\end{tabular} & $\begin{array}{l}0.23 \\
0.06\end{array}$ & $\begin{array}{l}0.31 \\
0.14\end{array}$ & & $\begin{array}{l}0.33 \\
0.24\end{array}$ & \begin{tabular}{|l|}
0.41 \\
0.36 \\
\end{tabular} & $\begin{array}{l}0.27 \\
0.13\end{array}$ & 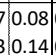 & $\begin{array}{l}0.39 \\
0.05\end{array}$ & $\begin{array}{l}0.06 \\
0.01\end{array}$ & \begin{tabular}{|l|}
0.36 \\
0.04
\end{tabular} & $\begin{array}{l}0.30 \\
0.04\end{array}$ & \begin{tabular}{|l|l|}
0.21 \\
0.05
\end{tabular} & \begin{tabular}{|l|}
0.16 \\
0.08
\end{tabular} & $\begin{array}{l}0.20 \\
0.14\end{array}$ & $\begin{array}{l}0.20 \\
0.25\end{array}$ & \begin{tabular}{|l|}
0.13 \\
0.16
\end{tabular} & $\begin{array}{l}0.42 \\
0.25\end{array}$ & \begin{tabular}{l|l}
0.10 & 0 \\
0.20 & 0
\end{tabular} & \begin{tabular}{|l|}
0.25 \\
0.04
\end{tabular} & \begin{tabular}{|l|}
0.09 \\
0.02
\end{tabular} & $\begin{array}{l}0.25 \\
0.04\end{array}$ & \begin{tabular}{|l|l|}
0.14 & 0 \\
0.02 & 0
\end{tabular} & \begin{tabular}{|l|}
0.22 \\
0.06
\end{tabular} & & & \begin{tabular}{|l|}
0.18 \\
0.11
\end{tabular} & \begin{tabular}{|l|}
0.28 \\
0.21
\end{tabular} & $\begin{array}{l}0.30 \\
0.13\end{array}$ & \begin{tabular}{|l|}
0.10 \\
0.16
\end{tabular} \\
\hline Buy & $\begin{array}{l}0.19 \\
0.01 \\
\end{array}$ & $\begin{array}{l}90.13 \\
0.01 \\
\end{array}$ & $\begin{array}{l}0.15 \\
0.01 \\
\end{array}$ & & & & & & & & & $\begin{array}{l}0.13 \\
0.01\end{array}$ & $\begin{array}{l}0.09 \\
0.01\end{array}$ & \begin{tabular}{l|}
0.11 \\
0.01
\end{tabular} & & & & & & & & & \begin{tabular}{|l|}
0.20 \\
0.02 \\
\end{tabular} & \begin{tabular}{|l|}
0.13 \\
0.02 \\
\end{tabular} & $\begin{array}{l}0.11 \\
0.01\end{array}$ & & & & & & & & \\
\hline Sell & & & & & & & & & & & \begin{tabular}{|l|}
0.07 \\
0.06 \\
\end{tabular} & \begin{tabular}{l|}
0.08 \\
0.01
\end{tabular} & & & & & & & & & & \begin{tabular}{l|}
0.06 \\
0.12 \\
\end{tabular} & & & & & & & & & & & \begin{tabular}{|l|}
0.08 \\
0.07 \\
\end{tabular} \\
\hline BA1* & $\begin{array}{l}0.17 \\
0.05 \\
\end{array}$ & \begin{tabular}{|l|l|}
5.16 \\
5.06 \\
\end{tabular} & $\begin{array}{l}0.29 \\
0.08 \\
\end{array}$ & \begin{tabular}{|l|}
0.24 \\
0.08 \\
\end{tabular} & $\begin{array}{l}0.17 \\
0.11 \\
\end{array}$ & \begin{tabular}{|l|}
0.10 \\
0.11 \\
\end{tabular} & & & & & & \begin{tabular}{|l|l}
0.12 & 0 \\
0.03 & 0 \\
\end{tabular} & $\begin{array}{l}0.17 \\
0.05\end{array}$ & $\begin{array}{l}0.48 \\
0.10 \\
\end{array}$ & $\begin{array}{l}0.29 \\
0.08\end{array}$ & \begin{tabular}{|l|}
0.18 \\
30.09 \\
\end{tabular} & \begin{tabular}{|l|}
0.10 \\
0.10 \\
\end{tabular} & \begin{tabular}{|l|}
0.08 \\
0.10 \\
\end{tabular} & & & & & \begin{tabular}{l|l}
0.15 \\
0.06 \\
\end{tabular} & \begin{tabular}{|l|}
0.11 \\
0.06 \\
\end{tabular} & $\begin{array}{l}0.28 \\
0.11 \\
\end{array}$ & \begin{tabular}{|l|l}
0.23 & 0 \\
0.10 & 0 \\
\end{tabular} & \begin{tabular}{|l|}
0.12 \\
0.09 \\
\end{tabular} & $\begin{array}{l}0.14 \\
0.13 \\
\end{array}$ & \begin{tabular}{|l|}
0.10 \\
0.12 \\
\end{tabular} & & & & \\
\hline AA1* & & & \begin{tabular}{|l|}
0.04 \\
0.01 \\
\end{tabular} & & & & & & & & & & & & & & & & & \begin{tabular}{l|}
0.03 \\
0.08 \\
\end{tabular} & & & & & & & & & & & & & \\
\hline & AA1 & AA2 & $\mathrm{AA} 3$ & AA4 & AA5 & AA6 & AA7 & AA8 & AA9 & AA10 & AA11 & AA1 & AA2 & $\mathrm{AA} 3$ & AA4 & AA5 & AA6 & AA7 & AA8 & AA9 & AA10A & AA11 A & AA1 & AA2 & AA3 & AA4 A & AA5 & AA6 & AA7 & AA8 & & AA10 & AA11 \\
\hline Buy* & \begin{tabular}{|l|}
0.34 \\
0.04
\end{tabular} & $\begin{array}{l}0.06 \\
0.01\end{array}$ & \begin{tabular}{|l|}
0.28 \\
0.03
\end{tabular} & \begin{tabular}{|l|}
0.18 \\
0.03
\end{tabular} & $\begin{array}{l}0.21 \\
0.06\end{array}$ & $\begin{array}{l}0.30 \\
0.14\end{array}$ & & $\begin{array}{ll}0.34 \\
0.25\end{array}$ & \begin{tabular}{|l|}
0.40 \\
0.37
\end{tabular} & $\begin{array}{l}0.27 \\
0.13\end{array}$ & $\begin{array}{l}0.08 \\
0.15\end{array}$ & \begin{tabular}{|l|l|l|}
0.42 & 0 \\
0.06 & 0 \\
\end{tabular} & \begin{tabular}{|l|l|}
0.14 & 0 \\
0.02 & 0
\end{tabular} & \begin{tabular}{|l|}
0.33 \\
0.03
\end{tabular} & $\begin{array}{l}0.26 \\
0.03\end{array}$ & $\begin{array}{l}0.17 \\
0.04\end{array}$ & \begin{tabular}{|l|}
0.21 \\
0.10
\end{tabular} & \begin{tabular}{|l|}
0.26 \\
0.18
\end{tabular} & $\begin{array}{l}0.25 \\
0.28\end{array}$ & $\begin{array}{l}0.13 \\
0.15\end{array}$ & $\begin{array}{l}0.42 \\
0.26\end{array}$ & \begin{tabular}{l|l}
0.09 & 0 \\
0.21 & 0
\end{tabular} & \begin{tabular}{|l|l|}
0.24 \\
0.04
\end{tabular} & \begin{tabular}{|l|}
0.08 \\
0.02 \\
\end{tabular} & $\begin{array}{l}0.26 \\
0.04\end{array}$ & \begin{tabular}{|l|l|l|}
0.15 & 0 \\
0.03 & 0 \\
\end{tabular} & \begin{tabular}{|l|}
0.23 \\
0.06
\end{tabular} & & \begin{tabular}{|l|}
0.28 \\
0.13 \\
\end{tabular} & \begin{tabular}{|l|}
0.17 \\
0.11 \\
\end{tabular} & \begin{tabular}{|l|}
0.29 \\
0.22
\end{tabular} & \begin{tabular}{l|}
0.30 \\
0.13
\end{tabular} & \begin{tabular}{|l|}
0.09 \\
0.16
\end{tabular} \\
\hline Sell* & $\begin{array}{l}0.45 \\
0.06 \\
\end{array}$ & $\begin{array}{l}0.34 \\
0.05 \\
\end{array}$ & \begin{tabular}{|l|}
0.32 \\
0.04 \\
\end{tabular} & \begin{tabular}{|l|}
0.20 \\
0.03 \\
\end{tabular} & $\begin{array}{l}0.19 \\
0.05\end{array}$ & & & & & & & $\begin{array}{l}0.45 \\
0.06 \\
\end{array}$ & $\begin{array}{l}0.37 \\
0.05 \\
0\end{array}$ & \begin{tabular}{l|}
0.34 \\
0.03 \\
\end{tabular} & $\begin{array}{l}0.17 \\
0.02\end{array}$ & & \begin{tabular}{|l|}
0.08 \\
0.03 \\
\end{tabular} & & & & & & \begin{tabular}{|l|}
0.44 \\
0.07 \\
\end{tabular} & \begin{tabular}{|l|}
0.37 \\
0.07 \\
\end{tabular} & $\begin{array}{l}0.41 \\
0.06 \\
\end{array}$ & & \begin{tabular}{|l|l|}
0.20 \\
0.05 \\
\end{tabular} & $\begin{array}{l}0.19 \\
0.07 \\
\end{array}$ & & & & & \\
\hline Buy & & & & & & & & & & & & \begin{tabular}{|l|}
0.13 \\
0.01 \\
\end{tabular} & & & & & & & & & & & & & & & & & & & & & \\
\hline Sell & $\begin{array}{l}0.20 \\
0.01 \\
\end{array}$ & $\begin{array}{l}0.12 \\
0.01 \\
\end{array}$ & & & & & & & & & & $\begin{array}{l}0.15 \\
0.01 \\
0\end{array}$ & \begin{tabular}{|l|}
0.10 \\
0.01 \\
\end{tabular} & & & & & & & & & & \begin{tabular}{|l|}
0.28 \\
0.02 \\
\end{tabular} & \begin{tabular}{|l|}
0.13 \\
0.01 \\
\end{tabular} & \begin{tabular}{l|}
0.18 \\
0.01 \\
\end{tabular} & & & & & & & & \\
\hline BA1* & & & \begin{tabular}{|l|}
0.05 \\
0.01 \\
\end{tabular} & & & & & & & & & & \begin{tabular}{|l|}
0.05 \\
0.02 \\
\end{tabular} & & & & & & & \begin{tabular}{|l|}
0.04 \\
0.09 \\
\end{tabular} & & & & & & & & & & & & & \\
\hline AA1* & $\begin{array}{l}0.17 \\
0.05 \\
\end{array}$ & $\begin{array}{l}0.17 \\
0.06 \\
\end{array}$ & \begin{tabular}{|l|}
0.32 \\
0.09 \\
\end{tabular} & \begin{tabular}{|l|}
0.23 \\
0.08 \\
\end{tabular} & $\begin{array}{l}0.16 \\
0.11 \\
\end{array}$ & \begin{tabular}{|l|}
0.09 \\
0.10 \\
\end{tabular} & & & & & & \begin{tabular}{l|}
0.14 \\
0.04 \\
0
\end{tabular} & $\begin{array}{l}0.19 \\
0.06\end{array}$ & $\begin{array}{l}0.49 \\
0.10 \\
\end{array}$ & $\begin{array}{l}0.28 \\
0.08\end{array}$ & $\begin{array}{l}0.20 \\
0.08\end{array}$ & \begin{tabular}{|l|}
0.11 \\
0.10 \\
\end{tabular} & & & & & & \begin{tabular}{|l|}
0.15 \\
0.06 \\
\end{tabular} & \begin{tabular}{|l|}
0.12 \\
0.06 \\
\end{tabular} & 0.270 & \begin{tabular}{|l|l|l|}
0.22 & 0 \\
0.10 & 0 \\
\end{tabular} & \begin{tabular}{|l|}
0.11 \\
0.08 \\
\end{tabular} & $\begin{array}{l}0.13 \\
0.12\end{array}$ & \begin{tabular}{l|}
0.09 \\
0.10
\end{tabular} & & & & \\
\hline & BC1 & $\mathrm{BC2}$ & $\mathrm{BC} 3$ & $\mathrm{BC4}$ & BC5 & BC6 & BC7 & BC8 & BC9 & $B C 10$ & BC11 & BC1 & $\mathrm{BC} 2$ & $\mathrm{BC} 3$ & $B C 4$ & BC5 & $\mathrm{BC6}$ & BC7 & $\mathrm{BC} 8$ & BC9 & BC10 & BC11 & BC1 & $\mathrm{BC2}$ & BC3 & $\mathrm{BC} 4 \mathrm{~B}$ & BC5 & $B C 6$ & BC7 & BC8 & & $\mathrm{BC} 10$ & $\mathrm{BC} 11$ \\
\hline Buy* & & $\begin{array}{l}0.12 \\
0.01 \\
\end{array}$ & \begin{tabular}{|l|}
0.13 \\
0.02 \\
\end{tabular} & \begin{tabular}{|l|}
0.15 \\
0.03 \\
\end{tabular} & $\begin{array}{l}0.14 \\
0.05 \\
\end{array}$ & $\begin{array}{l}0.14 \\
0.05\end{array}$ & & & \begin{tabular}{|l|}
0.13 \\
0.13 \\
\end{tabular} & $\begin{array}{l}0.15 \\
0.15 \\
\end{array}$ & $\begin{array}{l}0.19 \\
0.09 \\
\end{array}$ & & & \begin{tabular}{|l|}
0.10 \\
0.02
\end{tabular} & $\begin{array}{l}0.16 \\
0.03\end{array}$ & $\begin{array}{l}0.17 \\
0.04 \\
\end{array}$ & \begin{tabular}{|l|}
0.14 \\
0.05 \\
\end{tabular} & \begin{tabular}{|l|}
0.14 \\
0.10 \\
\end{tabular} & & & $\begin{array}{ll}0.14 \\
0.21\end{array}$ & \begin{tabular}{|l|}
0.12 \\
0.07 \\
\end{tabular} & & \begin{tabular}{|l|}
0.16 \\
0.02 \\
\end{tabular} & \begin{tabular}{l|}
0.17 \\
0.03
\end{tabular} & \begin{tabular}{|l|l|}
0.15 & 0 \\
0.03 & \\
\end{tabular} & \begin{tabular}{|l|}
0.16 \\
0.05 \\
\end{tabular} & $\begin{array}{l}0.11 \\
0.04\end{array}$ & & & & & $\begin{array}{l}0.20 \\
0.09 \\
\end{array}$ \\
\hline Sell* & $\begin{array}{l}0.80 \\
0.11 \\
\end{array}$ & \begin{tabular}{|l|}
0.84 \\
0.10 \\
\end{tabular} & \begin{tabular}{|l|}
0.55 \\
0.09 \\
\end{tabular} & \begin{tabular}{|l|}
0.24 \\
0.05 \\
\end{tabular} & $\begin{array}{l}0.19 \\
0.06\end{array}$ & 0.11 & $\begin{array}{l}0.14 \\
0.07 \\
\end{array}$ & 0.110 & \begin{tabular}{|l|}
0.08 \\
0.08 \\
\end{tabular} & & & \begin{tabular}{l|}
0.87 \\
0.11 \\
\end{tabular} & $\begin{array}{l}0.94 \\
0.10 \\
\end{array}$ & \begin{tabular}{|l|}
0.43 \\
0.07 \\
\end{tabular} & $\begin{array}{l}0.22 \\
0.04\end{array}$ & $\begin{array}{l}0.18 \\
0.05 \\
\end{array}$ & \begin{tabular}{|l|}
0.16 \\
0.06 \\
\end{tabular} & \begin{tabular}{|l|}
0.10 \\
0.07 \\
\end{tabular} & $\begin{array}{l}0.10 \\
0.10 \\
\end{array}$ & & & & \begin{tabular}{|l|}
0.75 \\
0.11 \\
\end{tabular} & \begin{tabular}{|l|}
0.82 \\
0.12 \\
\end{tabular} & \begin{tabular}{l|}
0.49 \\
0.09
\end{tabular} & $\begin{array}{lll}0.27 & 0 \\
0.06 & 0 \\
\end{array}$ & \begin{tabular}{|l|}
0.23 \\
0.07 \\
\end{tabular} & $\begin{array}{l}0.16 \\
0.05\end{array}$ & \begin{tabular}{|l|l|}
0.09 & \\
0.05 & \\
\end{tabular} & \begin{tabular}{|l|l|}
0.12 & \\
0.08 & \\
\end{tabular} & \begin{tabular}{|l|}
0.08 \\
0.07 \\
\end{tabular} & & \\
\hline Buy & & & & & & & & & & & & & & & & & & & & & & & & & & & & & & & & & \\
\hline Sell & $\begin{array}{l}0.20 \\
0.01 \\
\end{array}$ & $\begin{array}{l}0.16 \\
0.01\end{array}$ & \begin{tabular}{|l|}
0.16 \\
0.01 \\
\end{tabular} & \begin{tabular}{|l|}
0.13 \\
0.01 \\
\end{tabular} & $\begin{array}{l}0.09 \\
0.02 \\
\end{array}$ & $\begin{array}{l}0.08 \\
0.02 \\
\end{array}$ & $\begin{array}{l}0.08 \\
0.02\end{array}$ & $\begin{array}{l}0.07 \\
0.03 \\
\end{array}$ & & & & \begin{tabular}{|l|l|}
0.16 \\
0.01 \\
\end{tabular} & \begin{tabular}{|l|l|}
0.18 & 0 \\
0.01 & 0.1 \\
\end{tabular} & \begin{tabular}{|l|}
0.13 \\
0.01 \\
\end{tabular} & $\begin{array}{l}0.08 \\
0.01\end{array}$ & $\begin{array}{l}0.07 \\
0.01\end{array}$ & & & & & & & \begin{tabular}{|l|l}
0.21 \\
0.02
\end{tabular} & \begin{tabular}{|l|}
0.15 \\
0.01 \\
\end{tabular} & 0.150 & \begin{tabular}{|l|}
0.10 \\
0.01 \\
\end{tabular} & & $\begin{array}{l}0.09 \\
0.02\end{array}$ & & & & & \\
\hline BA1* & & $\begin{array}{l}0.21 \\
0.06 \\
\end{array}$ & \begin{tabular}{|l|}
0.10 \\
0.04 \\
\end{tabular} & \begin{tabular}{|l|}
0.25 \\
0.12 \\
\end{tabular} & $\begin{array}{l}0.12 \\
0.09 \\
\end{array}$ & 0.36 & $\begin{array}{l}0.28 \\
0.33 \\
\end{array}$ & $\begin{array}{l}0.17 \\
0.32\end{array}$ & \begin{tabular}{|l|}
0.15 \\
0.33 \\
\end{tabular} & $\begin{array}{l}0.17 \\
0.41 \\
\end{array}$ & $\begin{array}{l}0.61 \\
0.65\end{array}$ & & \begin{tabular}{|l|}
0.23 \\
0.05 \\
0
\end{tabular} & \begin{tabular}{|l|}
0.10 \\
0.03
\end{tabular} & $\begin{array}{l}0.30 \\
0.11\end{array}$ & $\begin{array}{l}0.19 \\
0.10\end{array}$ & \begin{tabular}{|l|}
0.34 \\
0.26 \\
\end{tabular} & \begin{tabular}{|l|}
0.23 \\
0.33 \\
\end{tabular} & $\begin{array}{l}0.16 \\
0.35 \\
\end{array}$ & $\begin{array}{l}0.10 \\
0.37\end{array}$ & 0.09 & \begin{tabular}{|l|}
0.59 \\
0.71 \\
\end{tabular} & & \begin{tabular}{|l|}
0.17 \\
0.07
\end{tabular} & 0.10 & \begin{tabular}{|l|l|}
0.22 & 0 \\
0.13 & 0 \\
\end{tabular} & \begin{tabular}{|l|}
0.10 \\
0.08 \\
\end{tabular} & $\begin{array}{l}0.22 \\
0.21 \\
\end{array}$ & \begin{tabular}{|l|l|}
0.19 \\
0.25 \\
\end{tabular} & \begin{tabular}{|l|l|}
0.15 & \\
0.27 & \\
\end{tabular} & \begin{tabular}{|l|}
0.12 \\
0.27 \\
\end{tabular} & \begin{tabular}{|l|}
0.15 \\
0.35
\end{tabular} & \begin{tabular}{l|}
0.61 \\
0.66 \\
\end{tabular} \\
\hline AA1* & $\begin{array}{l}0.29 \\
0.09 \\
\end{array}$ & $\begin{array}{l}0.17 \\
0.05 \\
\end{array}$ & $\begin{array}{l}0.15 \\
0.05 \\
\end{array}$ & \begin{tabular}{|l|}
0.10 \\
0.05 \\
\end{tabular} & $\begin{array}{l}0.07 \\
0.05 \\
\end{array}$ & $\begin{array}{l}0.04 \\
0.04\end{array}$ & & & & & & \begin{tabular}{|l|l|}
0.31 \\
0.08 \\
\end{tabular} & \begin{tabular}{|l|l}
0.23 & 0 \\
0.05 & 0 \\
\end{tabular} & \begin{tabular}{|l|}
0.14 \\
0.04 \\
\end{tabular} & $\begin{array}{l}0.07 \\
0.03 \\
\end{array}$ & & & & & & & & \begin{tabular}{|l|}
0.27 \\
0.10
\end{tabular} & \begin{tabular}{|l|}
0.18 \\
0.07 \\
\end{tabular} & \begin{tabular}{l|}
0.17 \\
0.08 \\
\end{tabular} & \begin{tabular}{|l|l|l|}
0.100 \\
0.06 \\
\end{tabular} & \begin{tabular}{|l|l|}
0.08 \\
0.06 \\
\end{tabular} & $\begin{array}{l}0.06 \\
0.06\end{array}$ & \begin{tabular}{|l|}
0.04 \\
0.06 \\
\end{tabular} & & & & \\
\hline & AC1 & $A C 2$ & $\mathrm{AC} 3$ & AC4 & AC5 & AC6 & AC7 & AC8 & AC9 & $A C 10$ & AC11 & AC1 & \begin{tabular}{|l|l}
$A C 2$ & $A$
\end{tabular} & $\mathrm{AC3}$ & AC4 & AC5 & AC6 & AC7 & AC8 & AC9 A & $\mathrm{AC} 10 \mathrm{~A}$ & AC11 A & AC1 & AC2 & AC3 & AC4 & AC5 & AC6 & AC7 & AC8 & AC9 A & AC10 & $\mathrm{AC} 11$ \\
\hline Buy* & $\begin{array}{l}0.81 \\
0.11 \\
\end{array}$ & $\begin{array}{l}0.85 \\
0.10\end{array}$ & \begin{tabular}{|l|}
0.52 \\
0.08 \\
\end{tabular} & \begin{tabular}{|l|}
0.22 \\
0.05 \\
\end{tabular} & $\begin{array}{l}0.18 \\
0.06\end{array}$ & 0.100 & $\begin{array}{l}0.13 \\
0.07\end{array}$ & \begin{tabular}{|l|l|}
0.11 & 0 \\
0.08 & 0 \\
\end{tabular} & \begin{tabular}{|l|}
0.08 \\
0.07 \\
\end{tabular} & & & \begin{tabular}{l|l|}
0.83 & 0 \\
0.11 & 0 \\
\end{tabular} & \begin{tabular}{|l|l|}
0.92 & 0 \\
0.10 & 0 \\
\end{tabular} & \begin{tabular}{|l|}
0.41 \\
0.06
\end{tabular} & $\begin{array}{l}0.23 \\
0.04\end{array}$ & $\begin{array}{l}0.160 \\
0.04\end{array}$ & \begin{tabular}{|l|}
0.15 \\
0.06 \\
\end{tabular} & \begin{tabular}{|l|}
0.11 \\
0.07 \\
\end{tabular} & & & & & \begin{tabular}{|l|}
0.74 \\
0.11 \\
\end{tabular} & \begin{tabular}{|l|}
0.79 \\
0.12 \\
\end{tabular} & $\begin{array}{ll}0.46 \\
0.08\end{array}$ & \begin{tabular}{|l|l|}
0.25 & 0 \\
0.06 & 0 \\
\end{tabular} & \begin{tabular}{l|c}
0.20 \\
0.06 \\
\end{tabular} & $\begin{array}{l}0.15 \\
0.05 \\
\end{array}$ & \begin{tabular}{|l|l|}
0.09 \\
0.05 \\
\end{tabular} & \begin{tabular}{|l|l|}
0.12 & \\
0.08 & \\
\end{tabular} & \begin{tabular}{|l|l|}
0.08 & \\
0.07 & \\
\end{tabular} & & \\
\hline Sell* & & $\begin{array}{l}0.14 \\
0.02 \\
\end{array}$ & \begin{tabular}{|l|}
0.12 \\
0.02 \\
\end{tabular} & \begin{tabular}{|l|}
0.14 \\
0.03 \\
\end{tabular} & $\begin{array}{l}0.15 \\
0.05 \\
\end{array}$ & \begin{tabular}{|l|}
0.14 \\
0.05 \\
\end{tabular} & & & \begin{tabular}{|l|}
0.11 \\
0.11 \\
\end{tabular} & $\begin{array}{l}0.16 \\
0.16 \\
\end{array}$ & \begin{tabular}{|l|}
0.19 \\
0.09 \\
\end{tabular} & & \begin{tabular}{|l|l}
0.15 & 0 \\
0.01 & 0 \\
\end{tabular} & \begin{tabular}{|l|}
0.13 \\
0.02 \\
\end{tabular} & $\begin{array}{l}0.16 \\
0.03 \\
\end{array}$ & \begin{tabular}{l|}
0.17 \\
0.04 \\
\end{tabular} & \begin{tabular}{|l|}
0.15 \\
0.05 \\
\end{tabular} & & & & \begin{tabular}{|l|l|}
0.15 & 0 \\
0.22 & 0 \\
\end{tabular} & \begin{tabular}{|l|}
0.13 \\
0.08 \\
\end{tabular} & & \begin{tabular}{|l|}
0.16 \\
0.02 \\
\end{tabular} & \begin{tabular}{l|l}
0.18 \\
0.03 \\
\end{tabular} & \begin{tabular}{|l|l}
0.200 \\
0.050 \\
\end{tabular} & \begin{tabular}{l|c}
0.17 \\
0.05 \\
\end{tabular} & $\begin{array}{l}0.11 \\
0.04 \\
\end{array}$ & & & \begin{tabular}{l|l}
0.14 & \\
0.12 & 0 \\
\end{tabular} & $\begin{array}{l}0.16 \\
0.14 \\
\end{array}$ & \begin{tabular}{|l|}
0.21 \\
0.09 \\
\end{tabular} \\
\hline Buy & $\begin{array}{l}0.20 \\
0.01 \\
\end{array}$ & $\begin{array}{l}0.15 \\
0.01\end{array}$ & \begin{tabular}{|l|}
0.14 \\
0.01 \\
\end{tabular} & \begin{tabular}{|l|}
0.11 \\
0.01
\end{tabular} & $\begin{array}{l}0.08 \\
0.01 \\
\end{array}$ & $\begin{array}{l}0.07 \\
0.01\end{array}$ & & & & & & \begin{tabular}{l|l}
0.17 \\
0.02
\end{tabular} & \begin{tabular}{|l|l|l|}
0.16 & 0 \\
0.01 & 0 \\
\end{tabular} & \begin{tabular}{|l|}
0.11 \\
0.01
\end{tabular} & $\begin{array}{l}0.12 \\
0.01\end{array}$ & \begin{tabular}{|l|l|}
0.07 \\
0.01
\end{tabular} & \begin{tabular}{|l|}
0.07 \\
0.02 \\
\end{tabular} & & & & & & \begin{tabular}{|l|}
0.21 \\
0.02
\end{tabular} & \begin{tabular}{|l|}
0.13 \\
0.01 \\
\end{tabular} & \begin{tabular}{|l|}
0.14 \\
0.01
\end{tabular} & \begin{tabular}{|l|}
0.09 \\
0.01 \\
\end{tabular} & & \begin{tabular}{|l|}
0.11 \\
0.02 \\
\end{tabular} & & & & & \\
\hline Sell & & & & & & & & & & & & & & & & & & & & & & & & & & & & & & & & & \\
\hline BA1* & $\begin{array}{l}0.30 \\
0.09 \\
\end{array}$ & $\begin{array}{l}0.18 \\
0.05 \\
\end{array}$ & \begin{tabular}{|l|}
0.15 \\
0.06 \\
\end{tabular} & \begin{tabular}{|l|}
0.09 \\
0.04 \\
\end{tabular} & $\begin{array}{l}0.06 \\
0.05 \\
\end{array}$ & $\begin{array}{l}0.04 \\
0.04\end{array}$ & & & & & & \begin{tabular}{l|l}
0.29 \\
0.08 \\
0
\end{tabular} & \begin{tabular}{|l|l|l|l|}
0.21 & 0 \\
0.05 & 0 \\
\end{tabular} & \begin{tabular}{|l|}
0.12 \\
0.04 \\
\end{tabular} & $\begin{array}{l}0.07 \\
0.02 \\
\end{array}$ & \begin{tabular}{|l|}
0.09 \\
0.04 \\
\end{tabular} & & & & & & & \begin{tabular}{|l|}
0.25 \\
0.10 \\
\end{tabular} & \begin{tabular}{|l|}
0.19 \\
0.07 \\
\end{tabular} & $\begin{array}{ll}0.17 \\
0.08\end{array}$ & \begin{tabular}{|l|l|}
0.10 & 0 \\
0.06 & 0 \\
\end{tabular} & \begin{tabular}{l|l|}
0.07 \\
0.06
\end{tabular} & $\begin{array}{l}0.07 \\
0.06\end{array}$ & \begin{tabular}{|l|}
0.05 \\
0.07 \\
\end{tabular} & & & & \\
\hline AA1* & & $\begin{array}{l}0.21 \\
0.06 \\
\end{array}$ & \begin{tabular}{|l|}
0.10 \\
0.04 \\
\end{tabular} & \begin{tabular}{|l|}
0.25 \\
0.12 \\
\end{tabular} & $\begin{array}{l}0.11 \\
0.09 \\
\end{array}$ & $\begin{array}{ll}0.35 \\
0.32\end{array}$ & $\begin{array}{l}0.28 \\
0.34 \\
\end{array}$ & \begin{tabular}{|l|l|}
0.18 & 0 \\
0.33 & 0 \\
\end{tabular} & \begin{tabular}{|l|}
0.13 \\
0.29 \\
\end{tabular} & $\begin{array}{l}0.16 \\
0.39 \\
\end{array}$ & $\begin{array}{l}0.60 \\
0.65\end{array}$ & & \begin{tabular}{|l|l|l|}
0.24 & 0 \\
0.05 & 0 \\
\end{tabular} & \begin{tabular}{|l|}
0.11 \\
0.03
\end{tabular} & $\begin{array}{l}0.27 \\
0.09 \\
\end{array}$ & \begin{tabular}{l|}
0.21 \\
0.10 \\
\end{tabular} & \begin{tabular}{|l|}
0.37 \\
0.27 \\
\end{tabular} & \begin{tabular}{|l|}
0.29 \\
0.37 \\
\end{tabular} & $\begin{array}{l}0.17 \\
0.35 \\
\end{array}$ & $\begin{array}{l}0.11 \\
0.37\end{array}$ & \begin{tabular}{|l|l|}
0.09 & 0 \\
0.28 & 0 \\
\end{tabular} & \begin{tabular}{|l|}
0.60 \\
0.71
\end{tabular} & & \begin{tabular}{|l|}
0.16 \\
0.06 \\
\end{tabular} & 0.100 & \begin{tabular}{|l|l|}
0.24 & 0 \\
0.15 & \\
\end{tabular} & $\begin{array}{l}0.10 \\
0.09 \\
\end{array}$ & $\begin{array}{l}0.21 \\
0.20 \\
\end{array}$ & \begin{tabular}{|l|l|l|l}
0.19 & \\
0.25 & \\
\end{tabular} & \begin{tabular}{|l|l|}
0.16 \\
0.28 \\
\end{tabular} & $\begin{array}{l}0.12 \\
0.27 \\
0.27\end{array}$ & \begin{tabular}{|l|}
0.14 \\
0.35
\end{tabular} & \begin{tabular}{|l|}
0.61 \\
0.66 \\
\end{tabular} \\
\hline
\end{tabular}




\section{Panel III: LOB Events effects on Bid LOS w/o BPI}

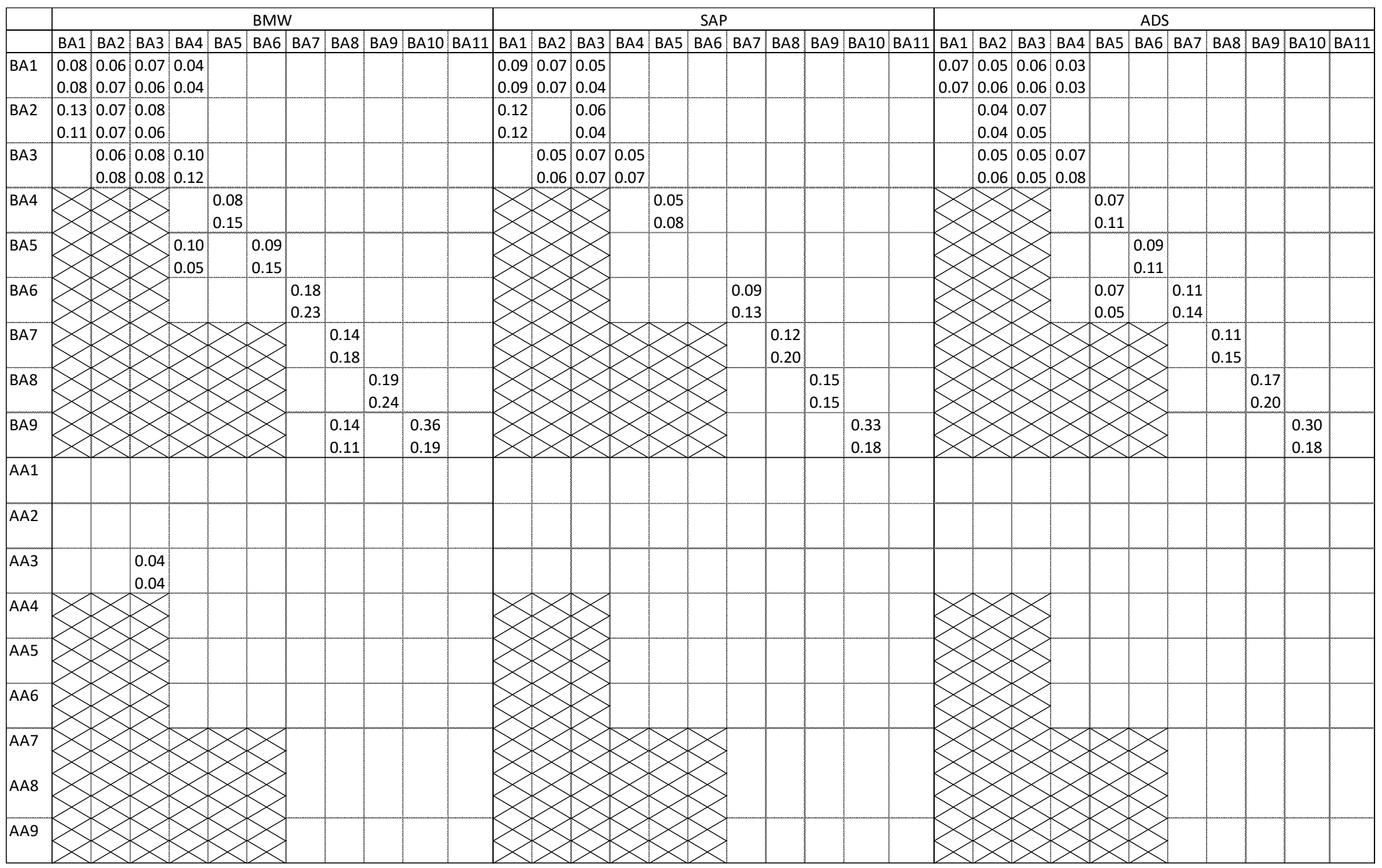




\section{Panel III: LOB Events effects on Bid LOS w/o BPI (cont.)}

\begin{tabular}{|c|c|c|c|c|c|c|c|c|c|c|c|c|c|c|c|c|c|c|c|c|c|c|c|c|c|c|c|c|c|c|c|c|c|}
\hline & \multicolumn{11}{|c|}{ BMW } & \multicolumn{11}{|c|}{ SAP } & \multicolumn{11}{|c|}{ ADS } \\
\hline & BA1 & \begin{tabular}{|l|}
$\mathrm{BA} 2$ \\
\end{tabular} & \begin{tabular}{|l|} 
BA3 \\
\end{tabular} & BA4 & BA5 & BA6 & BA7 & BA8 & BA9 & \begin{tabular}{|l|} 
BA10 \\
\end{tabular} & BA11 & BA1 & BA2 & BA3 & BA4 & BA5 & BA6 & \begin{tabular}{|l|} 
BA7 \\
\end{tabular} & BA8 & BA9 & BA10 & BA11 & BA1 & \begin{tabular}{|l|} 
BA2 \\
\end{tabular} & BA3 & \begin{tabular}{|l|} 
BA4 \\
\end{tabular} & BA5 & \begin{tabular}{|l|} 
BA6 \\
\end{tabular} & BA7 & \begin{tabular}{|l|} 
BA8 \\
\end{tabular} & \begin{tabular}{|l|} 
BA9 \\
\end{tabular} & BA10 & BA11 \\
\hline BC1 & 0 & \begin{tabular}{|l|}
0.03 \\
\end{tabular} & 0.06 & & & & & & & 0.05 & & 0.10 & 0.03 & 0.07 & 0.04 & & \begin{tabular}{|l|} 
\\
\end{tabular} & & & & \begin{tabular}{|l|} 
\\
\end{tabular} & & \begin{tabular}{|l|}
0.11 \\
\end{tabular} & 0.04 & $|0.07|$ & 0.03 & & & & \begin{tabular}{|l|} 
\\
\end{tabular} & & 0.06 & \\
\hline & 0.10 & 0.04 & 0.06 & & & & & & & 0.17 & & 0.11 & 0.03 & 0.05 & 0.04 & & 0.11 & & & & 0.20 & & 0.11 & 0.06 & 0.07 & 0.03 & & & & 0.14 & & 0.19 & \\
\hline BC2 & 0.07 & 0.02 & 0.06 & 0.06 & & & & & & & & 0.08 & 0.12 & 0.05 & 0.04 & & & & & 0.03 & & & 0.06 & 0.03 & 0.06 & 0.05 & & & & 0.04 & 0.05 & & \\
\hline & 0.08 & 0.03 & 0.06 & 0.07 & & & & & & & & 0.10 & 0.16 & 0.05 & 0.06 & & & & & 0.32 & & & 0.07 & 0.05 & 0.06 & 0.06 & & & & 0.16 & 0.25 & & \\
\hline BC3 & & 0.04 & 0.06 & 0.11 & & & & & & 0.05 & & & 0.06 & 0.31 & 0.11 & 0.05 & & & & & & & & 0.03 & & 0.14 & & & & & & 0.05 & \\
\hline & & 0.04 & 0.04 & 0.10 & & & & & & 0.15 & & & 0.06 & 0.21 & 0.09 & 0.08 & & & & & & & & 0.04 & & 0.12 & & & & & & 0.13 & \\
\hline BC4 & & & & 0.08 & 0.08 & & & & & & 0.04 & & & & 0.43 & 0.07 & 0.04 & & & & & & & & & & 0.06 & & & & & & \begin{tabular}{|l|l|} 
\\
\end{tabular} \\
\hline & & & & 0.05 & 0.10 & & & & & & 0.33 & & & & 0.34 & 0.09 & 0.10 & & & & & & & & & & 0.07 & & & & & & 0.29 \\
\hline BC5 & & & & 0.08 & 0.23 & & & & & & & & & & & 0.40 & & & & & & & & & & & 0.21 & 0.07 & & & & & \\
\hline & & & & 04 & 0.19 & & & & & & & & & & & 0.37 & & & & & & & & & & 0.04 & 0.18 & 0.08 & & & & & \\
\hline BC6 & & & & 0.22 & & 0.20 & & & & & & & & & & & 0.31 & & & & & & & & & 0.19 & & 0.36 & & & & & \\
\hline & & & & & & 0.24 & & & & & & & & & & & 0.38 & & & & & & & & & 0.05 & & 0.35 & & & & & \\
\hline & & & & & & & & & & & & & & & & & & & & & & & & & & & & & & & & & \\
\hline BC8 & & & & & & & & 0.11 & & & & & & & & & & & 0.10 & & & & & & & & & & & 0.13 & & & \\
\hline & & & & & & & & 0.10 & & & & & & & & & & & & & & & & & & & & & & 0.11 & & & \\
\hline $\mathrm{AC1}$ & 0.09 & 0.05 & 0.07 & 0.05 & & & & & & & & 0.08 & 0.07 & 0.07 & 0.04 & & & & & & & & 0.09 & 0.05 & 0.08 & 0.05 & 0.04 & & & & & & \\
\hline & 0.09 & $0.07 \mid$ & 0.07 & 0.06 & & & & & & & & 0.08 & 0.07 & 0.05 & 0.04 & & & & & & & & 0.09 & 0.07 & 0.08 & 0.06 & 0.08 & & & & & & \\
\hline$A C 2$ & 0.06 & $\mid$\begin{tabular}{|l|} 
\\
\end{tabular} & 0.05 & & & & & & & & & $\mid 0.07$ & 0.07 & 0.06 & & & & & & & & & 0.08 & 0.04 & \begin{tabular}{|l|} 
\\
\end{tabular} & 0.03 & & & & & & & \\
\hline & 0.06 & 0.05 & 0.05 & & & & & & & & & 0.09 & 0.09 & 0.06 & & & & & & & & & $\mid 0.08$ & | 0.06 & 0.05 & $\mid 0.04$ & & & & & & & \\
\hline AC3 & 0.09 & 0.06 & 0.09 & 0.05 & & & & & & & & & 0.14 & 0.12 & & & & & & & & & \begin{tabular}{|l|}
0.11 \\
\end{tabular} & 0.05 & 0.06 & 0.05 & & & & & & & \\
\hline & 0.08 & 0.06 & 0.07 & 0.04 & & & & & & & & & 0.13 & 0.08 & & & & & & & & & 0.09 & 0.06 & 0.05 & 0.04 & & & & & & & \\
\hline AC4 & & & & & & & & & & & & & & & & & & & & & & & & & & 0.05 & & & & & & & \\
\hline AC5 & & & & & & & & & & & & & & & & & & & & & & & & & & & & & & & & & \\
\hline AC6 & & & & & & & & & & & & & & & & & & & & & & & & & & & & & & & & & \\
\hline AC7 & & & & & & & & & & & & & & & & & & & & & & & & & & & & & & & & & \\
\hline AC8 & & & & & & & & & & & & & & & & & & & & & & & & & & & & & & & & & \\
\hline AC9 & & & & & & & & & & & & & & & & & & & & & & & & & & & & & & & & & \\
\hline ACIO & & & & & & & & & & & & & & & & & & & & & & & & & & & & & & & & & \\
\hline
\end{tabular}




\section{Panel IV: LOB Events effects on Ask LOS w/ BPI}

\begin{tabular}{|c|c|c|c|c|c|c|c|c|c|c|c|c|c|c|c|c|c|c|c|c|c|c|c|c|c|c|c|c|c|c|c|c|c|}
\hline & \multicolumn{11}{|c|}{ BMW } & \multicolumn{11}{|c|}{ SAP } & \multicolumn{11}{|c|}{ ADS } \\
\hline & AA1 & AA2 & AA3 & AA4 & AA5 & AA6 & \begin{tabular}{|l|} 
AA7 \\
\end{tabular} & AA8 & AA9 & AA10 & AA11 & AA1 & AA2 & AA3 & AA4 & AA5 & AA6 & \begin{tabular}{|l|} 
AA7 \\
\end{tabular} & AA8 & AA9 & AA10 & AA11 & AA1 & AA2 & AA3 & AA4 & AA5 & AA6 & AA7 & AA8 & AA9 & AA10 & AA11 \\
\hline BA1 & & & & & & & & & & & & & & & & & & & & & & & & & & & & & & & & & \\
\hline BA2 & & & & & & & & & & & & & & & & & & & & & & & & & & & & & & & & & \\
\hline BA3 & & & $\begin{array}{l}0.04 \\
0.04\end{array}$ & & & & & & & & & & & \begin{tabular}{|l|}
0.03 \\
0.03
\end{tabular} & & & & & & & & & & & & & & & & & & & \\
\hline BA4 & & & & & & & & & & & & & & & & & & & & & & & & & & & & & & & & & \\
\hline BA5 & & & & & & & & & & & & & & & & & & & & & & & & & & & & & & & & & \\
\hline BA6 & & & & & & & & & & & & & & & & & & & & & & & & & & & & & & & & & \\
\hline BA7 & & & & & & & & & & & & & & & & & & & & & & & & & & & & & & & & & \\
\hline BA8 & & & & & & & & & & & & & & & & & & & & & & & & & & & & & & & & & \\
\hline BA9 & & & & & & & & & & & & & & & & & & & & & & & & & & & & & & & & & \\
\hline AA1 & 0.08 & 0.06 & 0.07 & 0.03 & & & & & & & & \begin{tabular}{|l|}
0.10 \\
\end{tabular} & \begin{tabular}{|l|} 
\\
\end{tabular} & 0.05 & & & & & & & & & 0.09 & 0.05 & 0.06 & 0.03 & & & & & & & \\
\hline & 0.08 & \begin{tabular}{l|l}
3 & 0.07
\end{tabular} & 0.06 & 0.04 & & & & & & & & 0.10 & 0.07 & 0.04 & & & & & & & & & 0.09 & 0.06 & 0.05 & 0.03 & & & & & & & \\
\hline AA2 & 0.10 & 0.07 & 0.08 & & & & & & & & & 0.12 & & 0.07 & & & & & & & & & & 0.05 & 0.07 & & & & & & & & \\
\hline & 0.08 & \begin{tabular}{ll|}
3 & 0.07 \\
\end{tabular} & 0.06 & & & & & & & & & 0.11 & & 0.05 & & & & & & & & & & 0.05 & 0.05 & & & & & & & & \\
\hline AA3 & 0.10 & 0.06 & 0.08 & 0.08 & & & & & & & & & $\mid 0.04$ & 0.07 & 0.05 & & & & & & & & & 0.05 & 0.05 & 0.08 & & & & & & & \\
\hline & 0.11 & 0.08 & 0.08 & \begin{tabular}{|l|l|l} 
& 0.10
\end{tabular} & & & & & & & & & 0.06 & 0.07 & 0.06 & & & & & & & & & 0.07 & 0.05 & 0.09 & & & & & & & \\
\hline AA4 & & & & & $\begin{array}{l}0.07 \\
0.13\end{array}$ & & & & & & & & & & & \begin{tabular}{|l|}
0.05 \\
0.08
\end{tabular} & & & & & & & & & & & \begin{tabular}{|l|}
0.06 \\
0.11
\end{tabular} & & & & & & \\
\hline AA5 & & & & $\begin{array}{l}0.09 \\
0.05\end{array}$ & & \begin{tabular}{|l|}
0.11 \\
0.18
\end{tabular} & & & & & & & & & & & & & & & & & & & & & & $\begin{array}{l}0.09 \\
0.11\end{array}$ & & & & & \\
\hline AA6 & & & & & & & $\begin{array}{l}0.16 \\
0.20\end{array}$ & & & & & & & & & & & & & & & & & & & & & & \begin{tabular}{|l|}
0.10 \\
0.13
\end{tabular} & & & & \\
\hline AA7 & & & & & & & & 0.16 & & & & & & & & & & & & & & & & & & & & & & 0.11 & & & \\
\hline AA8 & & & & & & & & 0.20 & 0.18 & & & & & & & & & & & 0.18 & & & & & & & & & & 0.15 & 0.17 & & \\
\hline & & & & & & & & & 0.22 & & & & & & & & & & & 0.17 & & & & & & & & & & & 0.19 & & \\
\hline AA9 & & & & & & & & & & \begin{tabular}{|l|}
0.36 \\
0.18
\end{tabular} & & & & & & & & & & & \begin{tabular}{|l|}
0.29 \\
0.16
\end{tabular} & & & & & & & & & & & \begin{tabular}{|l|}
0.30 \\
0.17
\end{tabular} & \\
\hline
\end{tabular}




\section{Panel IV: LOB events effects on Ask LOS w/o BPI (cont.)}

\begin{tabular}{|c|c|c|c|c|c|c|c|c|c|c|c|c|c|c|c|c|c|c|c|c|c|c|c|c|c|c|c|c|c|c|c|c|c|}
\hline & \multicolumn{11}{|c|}{ BMW } & \multicolumn{11}{|c|}{ SAP } & \multicolumn{11}{|c|}{ ADS } \\
\hline & AA1 & AA2 & AA3 & AA4 & AA5 & AA6 & AA7 & AA8 & AA9 & AA10 & AA11 & AA1 & AA2 & AA3 & AA4 & AA5 & AA6 & AA7 & AA8 & AA9 & AA10 & AA11 & AA1 & AA2 & AA3 & AA4 & AA5 & AA6 & AA7 & AA8 & AA9 & AA10 & AA11 \\
\hline BC1 & 0.09 & 0.06 & 0.07 & 0.04 & & & & & & & & 0.08 & 0.06 & 0.07 & 0.04 & & & & & & & & 0.09 & 0.05 & 0.08 & 0.05 & 0.04 & & & & & & \\
\hline & 0.09 & 0.07 & 0.07 & 0.05 & & & & & & & & 0.09 & 0.07 & 0.05 & 0.04 & & & & & & & & 0.10 & 0.07 & 0.08 & 0.06 & 0.08 & & & & & & \\
\hline BC2 & $\mid 0.06$ & 0.05 & 0.05 & 0.03 & & & & & & & & 0.07 & 0.07 & 0.08 & & & & & & & & & & 0.04 & 0.05 & 0.04 & & & & & & & \\
\hline & \begin{tabular}{|l|}
0.06 \\
\end{tabular} & 0.06 & 0.05 & 0.04 & & & & & & & & 0.10 & 0.10 & 0.08 & & & & & & & & & & 0.06 & 0.05 & 0.04 & & & & & & & \\
\hline BC3 & 0.09 & 0.06 & 0.09 & 0.03 & & & & & & & & & 0.13 & 0.14 & & & & & & & & & 0.14 & 0.05 & 0.06 & 0.04 & & & & & & & \\
\hline & 0.07 & 0.07 & 0.07 & 0.03 & & & & & & & & & 0.12 & 0.09 & & & & & & & & & 0.12 & 0.06 & 0.05 & 0.04 & & & & & & & \\
\hline BC4 & & & & 0.05 & & & & & & & & & & & & & & & & & & & & & & & & & & & & & \\
\hline BC5 & & & & & & & & & & & & & & & & & & & & & & & & & & & & & & & & & \\
\hline BC6 & & & & & & & & & & & & & & & & & & & & & & & & & & & & & & & & & \\
\hline BC7 & & & & & & & & & & & & & & & & & & & & & & & & & & & & & & & & & \\
\hline BC8 & & & & & & & & & & & & & & & & & & & & & & & & & & 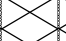 & & & & & & & \\
\hline BC9 & & & & & & & & & & & & & & & & & & & & & & & & & & & & & & & & & \\
\hline $\mathrm{AC1}$ & 0.11 & \begin{tabular}{|l|l|} 
\\
\end{tabular} & 0.06 & & & & & & & 0.05 & & 0.09 & \begin{tabular}{|l|l|}
0.03 \\
\end{tabular} & 0.06 & 0.04 & & & & & & \begin{tabular}{|l|}
0.04 \\
\end{tabular} & & 0.10 & .04 & 0.08 & & & & & 0.03 & & 0.07 & \\
\hline & 0.11 & 0.04 & 0.06 & & & & & & & 0.17 & & 0.10 & 0.03 & 0.04 & 0.04 & & & & & & 0.20 & & 0.10 & 0.06 & 0.07 & & & & & 0.13 & & 0.19 & \\
\hline $\mathrm{AC2}$ & & 0.03 & 0.06 & 0.05 & & & & & & & & 0.07 & 0.10 & 0.05 & 0.04 & & & & & 0.03 & & & 0.06 & 0.03 & 0.06 & 0.05 & & & & 0.03 & 0.05 & & \\
\hline & & 0.04 & 0.06 & 0.07 & & & & & & & & 0.09 & 0.14 & 0.05 & 0.05 & & & & & 0.32 & & & 0.07 & 0.04 & 0.06 & 0.05 & & & & 0.15 & 0.25 & & \\
\hline $\mathrm{AC3}$ & & 0.04 & 0.06 & 0.10 & & & & & & 0.04 & & & 0.04 & 0.28 & 0.09 & 0.05 & & & & & & & & 0.03 & 0.05 & 0.14 & & & & & & 0.05 & \\
\hline & & 0.04 & 0.05 & 0.10 & & & & & & 0.14 & & & 0.04 & 0.19 & 0.08 & 0.08 & & & & & & & & 0.04 & 0.04 & 0.13 & & & & & & 0.13 & \\
\hline $\mathrm{AC4}$ & & & & 0.06 & 0.08 & & & & & & 0.04 & & & & 0.41 & 0.06 & 0.05 & & & & & & & & & 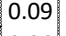 & 0.06 & & & & & & 0.04 \\
\hline & & & & 0.04 & 0.10 & & & & & & 0.36 & & & & 0.33 & 0.08 & 0.15 & & & & & & & & & 0.06 & 0.07 & & & & & & 0.31 \\
\hline AC5 & & & & 0.08 & 0.24 & & & & & & & & & & & 0.37 & & & & & & & & & & 0.07 & 0.19 & 0.07 & & & & & \\
\hline & & & & & 0.20 & & & & & & & & & & & 0.34 & & & & & & & & & & & 0.16 & 0.08 & & & & & \\
\hline AC6 & & & & 0.22 & & 0.17 & & & & & & & & & 0.15 & 0.11 & $0.27 \mid$ & & & & & & & & & 0.17 & 0.08 & 0.31 & & & & & \\
\hline & & & & 09 & & 0.21 & & & & & & & & & & 0.06 & 0.33 & & & & & & & & & & 0.06 & 0.30 & & & & & \\
\hline AC7 & & & & & & & 0.13 & & & & & & & & & & & & & & & & & & & & & & & & & & \\
\hline AC8 & & & & & & & & $\begin{array}{l}0.11 \\
0.11\end{array}$ & & & & & & & & & & & $\begin{array}{l}0.18 \\
0.19\end{array}$ & & & & & & & & & & & $\mid \begin{array}{l}0.11 \\
0.10\end{array}$ & & & \\
\hline AC9 & & & & & & & & & & & & & & & & & & & & & & & & & & & & & & & & & \\
\hline & & & & & & & & & & & & & & & & & & & & & & & & & & & & & & & & & \\
\hline
\end{tabular}




\section{Panel V: LOB events effects on Bid LOC}

\begin{tabular}{|c|c|c|c|c|c|c|c|c|c|c|c|c|c|c|c|c|c|c|c|c|c|c|c|c|c|c|c|c|c|c|c|c|c|}
\hline & \multicolumn{11}{|c|}{ BMW } & \multicolumn{11}{|c|}{ SAP } & \multicolumn{11}{|c|}{ ADS } \\
\hline & BC1 & $\mathrm{BC} 2$ & BC3 & BC4 & BC5 & BC6 & BC7 & BC8 & BC9 & BC10 & BC11 & BC1 & BC2 & BC3 & BC4 & BC5 & BC6 & BC7 & BC8 & BC9 & BC10 & BC11 & BC1 & $\mathrm{BC2}$ & BC3 & BC4 & BC5 & BC6 & BC7 & $B C 8$ & BC9 & BC10 & BC11 \\
\hline BA1 & $\begin{array}{l}0.13 \\
0.13\end{array}$ & $\begin{array}{l}0.03 \\
0.03\end{array}$ & & $\mid \begin{array}{l}0.03 \\
0.05\end{array}$ & & & & & & & & $\begin{array}{l}0.19 \\
0.19\end{array}$ & $\left|\begin{array}{l|}0.03 \\
0.02\end{array}\right|$ & $\left|\begin{array}{l}0.03 \\
0.03\end{array}\right|$ & & & & & & & & & $\begin{array}{l}0.15 \\
0.15\end{array}$ & $\mid$\begin{tabular}{l|}
0.03 \\
0.03
\end{tabular} & & & & & & & & & \\
\hline BA2 & & $\begin{array}{l}0.06 \\
0.04\end{array}$ & & & & & & & & & & & \begin{tabular}{|l|}
0.17 \\
0.13
\end{tabular} & & & & & & & & & & & & & & & & & & & & \\
\hline BA3 & & & $\begin{array}{l}0.07 \\
0.09\end{array}$ & & & & & & & & & & & $\begin{array}{l}0.15 \\
0.22 \\
\end{array}$ & & & & & & & & & & & & & & & & & & & \\
\hline BA4 & & & & & & & & & & & & & & & & & & & & & & & & & & & & & & & & & \\
\hline BA6 & & & & & & & & & & & & & & & & & \begin{tabular}{|l|}
0.34 \\
0.27 \\
\end{tabular} & & & & & & & & & & & & & & & & \\
\hline BA7 & & & & & & & & & & & & & & & & & & & & & & & & & & & & & & & & & \\
\hline BA8 & & & & & & & & & & & & & & & & & & & & & & & & & & & & & & & & & \\
\hline BA9 & & & & & & & & & & & & & & & & & & & & & & & & & & & & & & & & & \\
\hline AA1 & 0.06 & 0.05 & 0.05 & & & & & & & & & 0.07 & 0.06 & 0.03 & & & & & & & & & 0.06 & 05 & .04 & & & & & & & & \\
\hline & 0.06 & 0.05 & 0.06 & & & & & & & & & 0.06 & 0.04 & 0.04 & & & & & & & & & 0.06 & 0.04 & .05 & & & & & & & & \\
\hline AA2 & 0.04 & 0.04 & 0.04 & & & & & & & & & 0.05 & 0.09 & & & & & & & & & & 0.04 & 0.05 & 0.06 & & & & & & & & \\
\hline & 0.03 & 0.03 & 0.04 & & & & & & & & & 0.05 & 0.06 & & & & & & & & & & 0.03 & 0.03 & 0.05 & & & & & & & & \\
\hline AA3 & 0.07 & 0.06 & 0.06 & & & & & & & & & & 0.09 & & & & & & & & & & 0.05 & 0.06 & 0.06 & & & & & & & & \\
\hline & 0.07 & 0.06 & 0.07 & & & & & & & & & & 0.09 & & & & & & & & & & 0.05 & 0.06 & 0.08 & & & & & & & & \\
\hline AA4 & & & & & & & & & & & & & & & & & & & & & & & & & & & & & & & & & \\
\hline AA5 & & & & & & & & & & & & & & & & & & & & & & & & & & & & & & & & & \\
\hline AA6 & & & & & & & & & & & & & & & & & & & & & & & & & & & & & & & & & \\
\hline AA7 & & & & & & & & & & & & & & & & & & & & & & & & & & & & & & & & & \\
\hline AA8 & & & & & & & & & & & & & & & & & & & & & & & & & & & & & & & & & \\
\hline AA9 & & & & & & & & & & & & & & & & & & & & & & & & & & & & & & & & & \\
\hline
\end{tabular}




\section{Panel V: LOB events effects on Bid LOC (cont.)}

\begin{tabular}{|c|c|c|c|c|c|c|c|c|c|c|c|c|c|c|c|c|c|c|c|c|c|c|c|c|c|c|c|c|c|c|c|c|c|}
\hline & & & & & & BMV & & & & & & & & & & & SAP & & & & & & & & & & & ADS & & & & & \\
\hline & $\mathrm{BC} 1$ & $\mathrm{BC} 2$ & BC3 & $\mathrm{BC} 4$ & $\mathrm{BC5}$ & $\mathrm{BC6}$ & $\mathrm{BC7}$ & $\mathrm{BC} 8$ & BC9 & BC10 & BC11 & $\mathrm{BC} 1$ & $\mathrm{BC} 2$ & $\mathrm{BC} 3$ & BC4 & BC5 & $\mathrm{BC6}$ & BC7 & $\mathrm{BC} 8$ & BC9 & $\mathrm{BC} 10$ & BC11 & $\mathrm{BC} 1$ & $\mathrm{BC2}$ & $\mathrm{BC} 3$ & $\mathrm{BC4}$ & BC5 & BC6 & BC7 & $\mathrm{BC} 8$ & $\mathrm{BC} 9$ & $\mathrm{BC} 10$ & BC11 \\
\hline $\mathrm{BC} 1$ & 0.17 & 0.20 & 0.10 & 0.05 & 0.03 & & & & & & & 0.15 & 0.18 & 0.06 & 0.04 & & & & & & & & 0.16 & 0.18 & 0.10 & 0.05 & $\mid 0.04$ & & & & & & \\
\hline & 0.17 & 0.18 & 0.12 & 0.07 & 0.09 & & & & & & & 0.15 & 0.14 & 0.08 & 0.05 & & & & & & & & 0.16 & 0.18 & 0.12 & 0.07 & $\mid 0.09$ & & & & & & \\
\hline $\mathrm{BC} 2$ & 0.08 & 0.10 & 0.06 & 0.03 & & & & & & & & 0.06 & 0.09 & 0.06 & 0.05 & & & & & & & & 0.07 & 0.08 & 0.07 & 0.04 & \begin{tabular}{|l|}
0.03 \\
\end{tabular} & & & & & & \\
\hline & 0.09 & 0.10 & 0.08 & 0.06 & & & & & & & & 0.08 & 0.09 & 0.09 & 0.09 & & & & & & & & 0.07 & 0.08 & 0.09 & 0.06 & 0.07 & & & & & & \\
\hline BC3 & 0.07 & 0.09 & 0.08 & 0.06 & 0.04 & 0.03 & & & & & & 0.10 & 0.07 & 0.09 & 0.05 & 0.07 & & & & & & & 0.07 & 0.07 & 0.08 & 0.06 & \begin{tabular}{|l|}
0.04 \\
\end{tabular} & 0.04 & & & & & \\
\hline & 0.06 & 0.07 & 0.08 & 0.08 & 0.08 & 0.08 & & & & & & 0.08 & 0.05 & 0.09 & 0.06 & 0.12 & & & & & & & 0.06 & 0.06 & 0.08 & 0.08 & 0.06 & 0.08 & & & & & \\
\hline BC4 & & & & 0.06 & 0.04 & 0.05 & & & & & & & & & & & & & & & & & & & & 0.07 & $|0.07|$ & 0.05 & 0.04 & & & & \\
\hline & & & & 0.06 & 0.07 & 0.10 & & & & & & & & & & & & & & & & & & & & 0. & $\mid 0.09$ & 0.07 & 0.08 & & & & \\
\hline BC5 & & & & 0.14 & & 0.07 & & & & & & & & & .07 & & & & & & & & & & & 06 & & 0.05 & 0.04 & & & & \\
\hline & & & & $y$ & & 0.09 & & & & & & & & & 04 & & & & & & & & & & & 05 & & 0.06 & 0.06 & & & & \\
\hline BC6 & & & & & 0.08 & & 0.06 & & & & & & & & & & & & & & & & & & & & & & 0.05 & & & & \\
\hline & & & & & 0.07 & & 0.08 & & & & & & & & & & & & & & & & & & & & & & 0.06 & & & & \\
\hline $\mathrm{BC7}$ & & & & & & & 0.13 & & & & & & & & & & & & & & & & & & & & & & & & & & \\
\hline$B C 8$ & & & & & & & & & & & & & & & & & & & & & & & & & & & & & & & & & \\
\hline BC9 & & & & & & & & & & & & & & & & & & & & & & & & & & & & & & & & & \\
\hline $\mathrm{AC} 1$ & & 0.02 & & 0.04 & & & & & & & & & & & 0.04 & & & & & & & & & & & 0.03 & & & & & & & \\
\hline & & 0.02 & & 0.06 & & & & & & & & & & & 0.05 & & & & & & & & & & & 0.05 & & & & & & & \\
\hline AC2 & & & & & & & & & & & & & & & & & & & & & & & & & & & & & & & & & \\
\hline AC3 & & & \begin{tabular}{|l|}
0.04 \\
\end{tabular} & & & & & & & & & & & 0.10 & & & & & & & & & & & & & & & & & & & \\
\hline & & & 0.04 & & & & & & & & & & & 0.11 & & & & & & & & & & & & & & & & & & & \\
\hline AC4 & & & & & & & & & & & & & & & & & & & & & & & & & & & & & & & & & \\
\hline AC5 & & & & & & & & & & & & & & & & & & & & & & & & & & & & & & & & & \\
\hline AC6 & & & & & & & & & & & & & & & & & & & & & & & & & & & & & & & & & \\
\hline AC7 & & & & & & & & & & & & & & & & & & & & & & & & & & & & & & & & & \\
\hline AC8 & & & & & & & & & & & & & & & & & & & & & & & & & & & & & & & & & \\
\hline AC9 & & & & & & & & & & & & & & & & & & & & & & & & & & & & & & & & & \\
\hline $\mathrm{C10}$ & & & & & & & & & & & & & & & & & & & & & & & & & & & & & & & & & \\
\hline
\end{tabular}




\section{Panel VI: LOB events effects on Ask LOC}

\begin{tabular}{|c|c|c|c|c|c|c|c|c|c|c|c|c|c|c|c|c|c|c|c|c|c|c|c|c|c|c|c|c|c|c|c|c|c|}
\hline & \multicolumn{11}{|c|}{ BMW } & \multicolumn{11}{|c|}{ SAP } & \multicolumn{11}{|c|}{ ADS } \\
\hline & AC1 & $\mathrm{AC2}$ & AC3 & AC4 & AC5 & AC6 & AC7 & AC8 & AC9 & AC10 & AC11 & AC1 & $A C 2$ & AC3 & AC4 & AC5 & AC6 & AC7 & AC8 & AC9 & AC10 & AC11 & AC1 & $A C 2$ & AC3 & $\mathrm{AC4}$ & AC5 & AC6 & AC7 & $A C 8$ & AC9 & AC10 & AC11 \\
\hline BA1 & 0.07 & 0.06 & 0.05 & \begin{tabular}{|l|l} 
& 0.03 \\
\end{tabular} & & & & & & & & 0.07 & 0.06 & \begin{tabular}{|l|} 
\\
\end{tabular} & & & & & & & & & 0.06 & \begin{tabular}{|l|}
0.05 \\
\end{tabular} & 0.04 & & & & & & & & \\
\hline & 0.07 & 0.05 & 0.06 & \begin{tabular}{|l|l} 
& 0.04
\end{tabular} & & & & & & & & 0.07 & 0.05 & 0.04 & & & & & & & & & 0.06 & 0.05 & 0.05 & & & & & & & & \\
\hline BA2 & 0.05 & 0.04 & 0.04 & & & & & & & & & 0.04 & 0.09 & & & & & & & & & & 0.04 & 0.05 & 0.06 & & & & & & & & \\
\hline & 0.04 & 0.03 & 0.03 & & & & & & & & & 0.04 & 0.06 & & & & & & & & & & 0.03 & 0.03 & 0.05 & & & & & & & & \\
\hline BA3 & & 0.06 & 0.06 & & & & & & & & & & 0.08 & \begin{tabular}{|l|}
0.10 \\
\end{tabular} & & & & & & & & & 0.06 & 0.05 & 0.06 & & & & & & & & \\
\hline & & 0.06 & 0.08 & & & & & & & & & & 0.08 & 0.15 & & & & & & & & & 0.05 & 0.05 & 0.07 & & & & & & & & \\
\hline BA4 & & & & & & & & & & & & & & & & & & & & & & & & & & & & & & & & & \\
\hline BA5 & & & & & & & & & & & & & & & & & & & & & & & & & & & & & & & & & \\
\hline BA6 & & & & & & & & & & & & & & & & & & & & & & & & & & & & & & & & & \\
\hline BA7 & & & & & & & & & & & & & & & & & & & & & & & & & & & & & & & & & \\
\hline BA8 & & & & & & & & & & & & & & & & & & & & & & & & & & & & & & & & & \\
\hline BA9 & & & & & & & & & & & & & & & & & & & & & & & & & & & & & & & & & \\
\hline AA1 & 0.13 & 0.03 & & 0.03 & & & & & & & & |0.17 & & & & & & & & & & & \begin{tabular}{|l|l|}
0.16 \\
\end{tabular} & & & & & & & & & & \\
\hline & 0.13 & 0.03 & & 0.05 & & & & & & & & 0.17 & & & & & & & & & & & 0.15 & & & & & & & & & & \\
\hline $\mathrm{AA} 2$ & & $\begin{array}{l}0.05 \\
0.04\end{array}$ & & & & & & & & & & & $\begin{array}{l}0.17 \\
0.12\end{array}$ & & & & & & & & & & & & & & & & & & & & \\
\hline AA3 & & & \begin{tabular}{|l|}
0.07 \\
0.09
\end{tabular} & & & & & & & & & & & $\begin{array}{l}0.15 \\
0.22\end{array}$ & & & & & & & & & & & & & & & & & & & \\
\hline AA4 & & & & & & & & & & & & & & & & & & & & & & & & & & & & & & & & & \\
\hline AA5 & & & & & 0.17 & & & & & & & & & & & 0.31 & & & & & & & & & & & & & & & & & \\
\hline AA6 & & & & & & & & & & & & & & & & & 0.34 & & & & & & & & & & & & & & & & \\
\hline & & & & & & & & & & & & & & & & & 0.27 & & & & & & & & & & & & & & & & \\
\hline AA7 & & & & & & & & & & & & & & & & & & & & & & & & & & & & & & & & & \\
\hline AA8 & & & & & & & & & & & & & & & & & & & & & & & & & & & & & & & & & \\
\hline AA9 & & & & & & & & & & & & & & & & & & & & & & & & & & & & & & & & & \\
\hline
\end{tabular}




\section{Panel VI: LOB events effects on Ask LOC (cont.)}

\begin{tabular}{|c|c|c|c|c|c|c|c|c|c|c|c|c|c|c|c|c|c|c|c|c|c|c|c|c|c|c|c|c|c|c|c|c|c|}
\hline & \multicolumn{11}{|c|}{ BMW } & \multicolumn{11}{|c|}{ SAP } & \multicolumn{11}{|c|}{ ADS } \\
\hline & AC1 & AC2 & AC3 & AC4 & AC5 & AC6 & AC7 & AC8 & AC9 & AC10 & AC11 & AC1 & AC2 & AC3 & AC4 & AC5 & AC6 & AC7 & AC8 & AC9 & AC10 & AC11 & AC1 & AC2 & AC3 & AC4 & AC5 & AC6 & AC7 & AC8 & AC9 & $\mathrm{AC} 10$ & AC11 \\
\hline $\mathrm{BC} 1$ & & \begin{tabular}{|l|}
0.03 \\
0.02 \\
\end{tabular} & & \begin{tabular}{|l|}
0.03 \\
0.05 \\
\end{tabular} & & & & & & & & & & & \begin{tabular}{|l|}
0.04 \\
0.05 \\
\end{tabular} & & & & & & & & & & & & & & & & & & \\
\hline $\mathrm{BC} 2$ & & & & & & & & & & & & & & & & & & & & & & & & & & & & & & & & & \\
\hline $\mathrm{BC} 3$ & & & $\begin{array}{l}0.04 \\
0.05\end{array}$ & & & & & & & & & & & & & & & & & & & & & & & & & & & & & & \\
\hline BC4 & & & & & & & & & & & & & & & & & & & & & & & & & & & & & & & & & \\
\hline BC5 & & & & & & & & & & & & & & & & & & & & & & & & & & & & & & & & & \\
\hline BC6 & & & & & & & & & & & & & & & & & & & & & & & & & & & & & & & & & \\
\hline BC7 & & & & & & & & & & & & & & & & & & & & & & & & & & & & & & & & & \\
\hline BC8 & & & & & & & & & & & & & & & & & 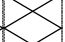 & & & & & & & & & & & & & & & & \\
\hline BC9 & & & & & & & & & & & & & & & & & & & & & & & & & & & & & & & & & \\
\hline $\mathrm{AC1}$ & 0.18 & 0.20 & 0.10 & 0.04 & 0.03 & & & & & & & 0.15 & .19 & 0.07 & 0.05 & & & & & & & & 0.17 & .19 & 10 & 0.05 & 0.04 & & & & & & \\
\hline & 0.18 & 0.18 & 0.12 & 0.06 & 0.09 & & & & & & & 0.15 & 14 & .08 & 0.06 & & & & & & & & 0.17 & 0.19 & 0.12 & 0.07 & 0.09 & & & & & & \\
\hline $\mathrm{AC2}$ & 0.09 & 0.10 & 0.06 & 0.03 & & & & & & & & 0.07 & 10 & 0.06 & 0.05 & & & & & & & & 0.07 & 0.08 & $0.07 \mid$ & 0.03 & 0.03 & & & & & & \\
\hline & 0.10 & 0.10 & 0.08 & 0.05 & & & & & & & & 0.09 & .10 & 0.09 & 0.07 & & & & & & & & 0.07 & 0.08 & 0.09 & 0.05 & 0.07 & & & & & & \\
\hline AC3 & 0.09 & 0.09 & 0.09 & 0.05 & 0.04 & 0.03 & & & & & & $\mid 0.10$ & 0.07 & 0.09 & 0.04 & 0.07 & & & & & & & 0.07 & 0.07 & 0.09 & 0.05 & 0.04 & 0.04 & & & & & \\
\hline & 0.07 & 0.07 & 0.09 & 0.07 & 0.08 & 0.08 & & & & & & 0.09 & 0.05 & 0.09 & 0.05 & 0.11 & & & & & & & 0.06 & 0.06 & 0.09 & 0.07 & 0.07 & 0.08 & & & & & \\
\hline AC4 & & & & 0.08 & 0.05 & 0.05 & & & & & & & & & & 0.04 & & & & & & & & & & 0.07 & 0.07 & 0.04 & 0.04 & & & & \\
\hline & & & & 0.08 & 0.08 & 0.10 & & & & & & & & & & 0.06 & & & & & & & & & & 0.07 & 0.09 & 0.07 & 0.08 & & & & \\
\hline AC5 & & & & 0.15 & & 0.07 & & & & & & & & & & & & & & & & & & & & & & 0.06 & 0.04 & & & & \\
\hline & & & & 0.09 & & 0.09 & & & & & & & & & & & & & & & & & & & & & & 0.06 & 0.06 & & & & \\
\hline AC6 & & & & & 0.08 & & 0.06 & & & & & & & & & & & & & & & & & & & & & & 0.04 & & & & \\
\hline & & & & & 0.07 & & 0.08 & & & & & & & & & & & & & & & & & & & & & & 0.05 & & & & \\
\hline AC7 & & & & & & & 0.14 & & & & & & & & & & & & & & & & & & & & & & & & & & \\
\hline AC8 & & & & & & & & & & & & & & & & & & & & & & & & & & & & & & & & & \\
\hline AC9 & & & & & & & & & & & & & & & & & & & & & & & & & & & & & & & & & \\
\hline AC10 & & & & & & & & & $\mid \begin{array}{l}0.15 \\
0.15\end{array}$ & & & & & & & & & & & & & & & & & & & & & & & & \\
\hline
\end{tabular}




\section{Table 6 Multi-events mutual-excitation examples}

Panel I: Levels 1 tot 3 Bid LOS w/o BPI and Ask LOC

\begin{tabular}{|l|l|l|l|l|l|l|}
\hline \multicolumn{7}{|c|}{} \\
\hline & BA1 & BA2 & BA3 & AC1 & AC2 & AC3 \\
\hline BA1 & 0.08 & 0.06 & 0.07 & 0.07 & 0.06 & 0.05 \\
& 0.08 & 0.07 & 0.06 & 0.07 & 0.05 & 0.06 \\
\hline BA2 & 0.13 & 0.07 & 0.08 & 0.05 & 0.04 & 0.04 \\
& 0.11 & 0.07 & 0.06 & 0.04 & 0.03 & 0.03 \\
\hline BA3 & & 0.06 & 0.08 & & 0.06 & 0.06 \\
& & 0.08 & 0.08 & & 0.06 & 0.08 \\
\hline AC1 & 0.09 & 0.05 & 0.07 & 0.18 & 0.20 & 0.10 \\
& 0.09 & 0.07 & 0.07 & 0.18 & 0.18 & 0.12 \\
\hline AC2 & 0.06 & 0.04 & 0.05 & 0.09 & 0.10 & 0.06 \\
& 0.06 & 0.05 & 0.05 & 0.10 & 0.10 & 0.08 \\
\hline AC3 & 0.09 & 0.06 & 0.09 & 0.09 & 0.09 & 0.09 \\
& 0.08 & 0.06 & 0.07 & 0.07 & 0.07 & 0.09 \\
\hline
\end{tabular}

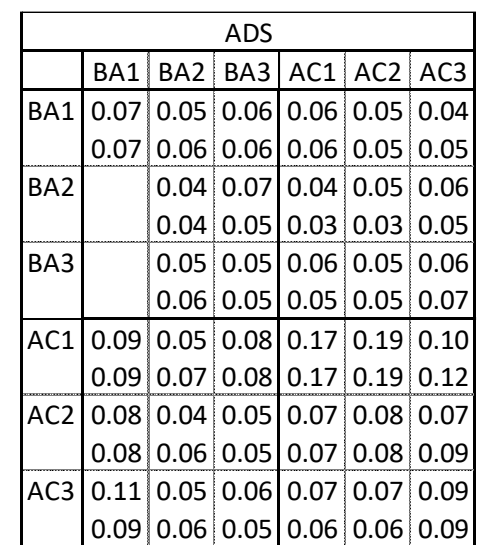

Panel II: Levels 1 tot 3 Ask LOS w/o BPI and Bid LOC

\begin{tabular}{|l|l|l|l|l|l|l|}
\hline \multicolumn{7}{|c|}{ BMW } \\
\hline & AA1 & AA2 & AA3 & BC1 & BC2 & BC3 \\
\hline AA1 & 0.08 & 0.06 & 0.07 & 0.06 & 0.05 & 0.05 \\
& 0.08 & 0.07 & 0.06 & 0.06 & 0.05 & 0.06 \\
\hline AA2 & 0.10 & 0.07 & 0.08 & 0.04 & 0.04 & 0.04 \\
& 0.08 & 0.07 & 0.06 & 0.03 & 0.03 & 0.04 \\
\hline AA3 & 0.10 & 0.06 & 0.08 & 0.07 & 0.06 & 0.06 \\
& 0.11 & 0.08 & 0.08 & 0.07 & 0.06 & 0.07 \\
\hline BC1 & 0.09 & 0.06 & 0.07 & 0.17 & 0.20 & 0.10 \\
& 0.09 & 0.07 & 0.07 & 0.17 & 0.18 & 0.12 \\
\hline BC2 & 0.06 & 0.05 & 0.05 & 0.08 & 0.10 & 0.06 \\
& 0.06 & 0.06 & 0.05 & 0.09 & 0.10 & 0.08 \\
\hline BC3 & 0.09 & 0.06 & 0.09 & 0.07 & 0.09 & 0.08 \\
& 0.07 & 0.07 & 0.07 & 0.06 & 0.07 & 0.08 \\
\hline
\end{tabular}

\begin{tabular}{|l|l|l|l|l|l|l|}
\hline \multicolumn{7}{|c|}{ ADS } \\
\hline & AA1 & AA2 & AA3 & BC1 & BC2 & BC3 \\
\hline AA1 & 0.09 & 0.05 & 0.06 & 0.06 & 0.05 & 0.04 \\
& 0.09 & 0.06 & 0.05 & 0.06 & 0.04 & 0.05 \\
\hline AA2 & & 0.05 & 0.07 & 0.04 & 0.05 & 0.06 \\
& & 0.05 & 0.05 & 0.03 & 0.03 & 0.05 \\
\hline AA3 & & 0.05 & 0.05 & 0.05 & 0.06 & 0.06 \\
& & 0.07 & 0.05 & 0.05 & 0.06 & 0.08 \\
\hline BC1 & 0.09 & 0.05 & 0.08 & 0.16 & 0.18 & 0.10 \\
& 0.10 & 0.07 & 0.08 & 0.16 & 0.18 & 0.12 \\
\hline BC2 & & 0.04 & 0.05 & 0.07 & 0.08 & 0.07 \\
& & 0.06 & 0.05 & 0.07 & 0.08 & 0.09 \\
\hline BC3 & 0.14 & 0.05 & 0.06 & 0.07 & 0.07 & 0.08 \\
& 0.12 & 0.06 & 0.05 & 0.06 & 0.06 & 0.08 \\
\hline
\end{tabular}

